\title{
Molecular modeling and experimental determination of the structure of C8 -arylguanine modified oligonucleotides that preferentially adopt the $Z$-DNA conformation
}

\author{
Sue Ellen Heavner \\ West Virginia University
}

Follow this and additional works at: https://researchrepository.wvu.edu/etd

\section{Recommended Citation}

Heavner, Sue Ellen, "Molecular modeling and experimental determination of the structure of C8 -arylguanine modified oligonucleotides that preferentially adopt the Z -DNA conformation" (2004). Graduate Theses, Dissertations, and Problem Reports. 2075.

https://researchrepository.wvu.edu/etd/2075

This Dissertation is protected by copyright and/or related rights. It has been brought to you by the The Research Repository @ WVU with permission from the rights-holder(s). You are free to use this Dissertation in any way that is permitted by the copyright and related rights legislation that applies to your use. For other uses you must obtain permission from the rights-holder(s) directly, unless additional rights are indicated by a Creative Commons license in the record and/ or on the work itself. This Dissertation has been accepted for inclusion in WVU Graduate Theses, Dissertations, and Problem Reports collection by an authorized administrator of The Research Repository @ WVU.

For more information, please contact researchrepository@mail.wvu.edu. 


\title{
Molecular Modeling and Experimental Determination of the Structure of C8- Arylguanine Modified Oligonucleotides that Preferentially Adopt the Z-DNA Conformation
}

\author{
Sue Ellen Heavner \\ Dissertation submitted to the \\ School of Pharmacy \\ at West Virginia University \\ in partial fulfillment of the requirements \\ for the degree of \\ Doctor of Philosophy \\ in \\ Pharmacy \\ Peter M. Gannett, Ph.D., Chair \\ John O’Donnell, Ph.D. \\ Eugene Demchuk, Ph.D., \\ Grazyna Szklarz, Ph.D., \\ Alan Stolzenberg, Ph.D. \\ Department of Basic Pharmaceutical Sciences \\ Morgantown, West Virginia \\ 2004
}

Keywords: Z-DNA, NMR, CD, quantum mechanics, molecular dynamics, carcinogenesis 


\section{Abstract \\ Molecular Modeling and Experimental Determination of the Structure of C8-Arylguanine Modified Oligonucleotides that Preferentially Adopt the Z-DNA Conformation}

\section{Sue Ellen Heavner}

Thermal denaturation, circular dichroism, and NMR have been used extensively in conformational studies of oligonucleotides. Molecular modeling has become a powerful, if not essential, complement to these experimental techniques. To study the effects of C8-arylated purine adducts caused by carcinogenic aryl hydrazines we combined the two approaches. Two oligonucleotides were prepared, an unmodified oligonucleotide $\left.\mathrm{d}^{5}{ }^{\prime} \mathrm{CGCGCGCGCG}^{3}\right)$ and a C8-phenylguanine modified oligonucleotide d( $\left.{ }^{5^{\prime}} \mathrm{CGCGCG}^{*} \mathrm{CGCG}^{3^{\prime}}\right)\left(\mathrm{G}^{*}=8\right.$-phenylguanine). These oligonucleotides were compared using thermal denaturation, circular dichroism, and NMR. The phenyl modification destabilizes the B DNA form and stabilizes the Z DNA form such that the B:Z ratio is near one under physiological conditions.

Modeling studies were conducted on the modified sequence with $G^{*}=$ guanine, $G^{*}=8$ phenylguanine, $\mathrm{G}^{*}=8$-tolylguanine, and $\mathrm{G}^{*}=8$-hydroxymethylphenylguanine in the $\mathrm{B}$ and $\mathrm{Z}$ DNA conformations. We performed quantum mechanical calculations at the B3LYP level with the standard 6-31G* basis set using Gaussian98, and molecular dynamics simulations and free energy calculations using the suite of programs contained in Amber 6 and 7 with the Cornell 95 force field. New parameters for the modified guanines $\left(\mathrm{G}^{*}\right)$ have been developed for the Cornell force field. Structural and thermodynamic properties of the DNA molecules are described from the analysis of the trajectories.

The phenyl and tolyl-modified DNA's favored the Z form and the B conformation was preferred by the unmodified and hydroxymethylphenyl-modified oligonucleotides. In those cases where experimental data was available, it was in agreement with the theoretical data. The phenyl-modified models were useful in supporting and providing explanation for the experimental results. In light of recent studies, which show a role for Z DNA in gene expression and cell transformation, Z DNA stabilization by $\mathrm{C} 8$-arylguanine formation from aryl hydrazines may be relevant to their role in carcinogenesis. 


\section{Acknowledgments}

First, I would like to express my sincerest thanks to Dr. Peter M. Gannett, my advisor, for giving me the opportunity to work with him. I have tremendous respect for Dr. Gannett; he has an incredible variety of interests and most of all, he never asks anyone to perform a task that he would not perform himself. I feel lucky to have him as an advisor. In addition, I would like to thank my committee members Dr. John O’Donnell, Dr. Eugene Demchuk, Dr. Grazyna Szklarz, and Dr. Alan Stolzenberg for finding time in their busy schedules to serve on my committee.

A very special thank you goes to Eva Darian. Without Eva, the computational portion of this work would not have been possible, or at least much more difficult. I also sincerely thank my colleagues Spencer Ericksen, Jonathan Daft, and Jeannine Powell, Spencer for his modeling expertise, and Jon and Jeannine for their contributions to various aspects of this work.

Many thanks go to all the people at Mylan who have helped make it possible for me to stand before you today. I can't possible acknowledge all of them, but I would like to point out a few: Mike Puskar and Dr. Tom Clark for seeing and believing in my potential, and Dr. Scott Chervenick for providing the means to accomplish this while at the same time advancing my career.

I would also like to thank my best friend, Sunki, for hanging in there, even when I have not been very much fun to be around. Thanks also to Bev and Sam Minor for letting me become a member of the "Springhouse family". Finally, I would like to thank my parents, not just for all they have done in support of achieving this goal, but also for a lifetime's supply of patience, encouragement, and love. 


\section{TABLE OF CONTENTS}

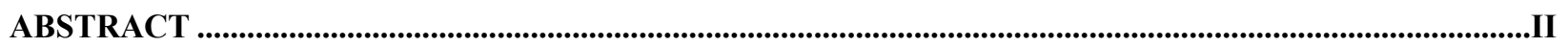

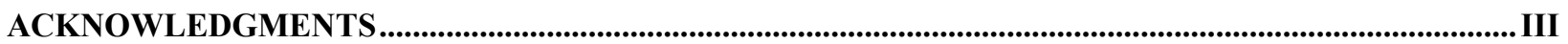

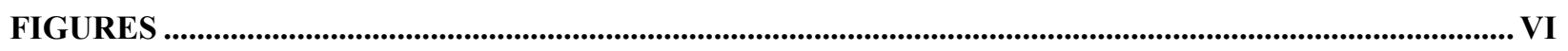

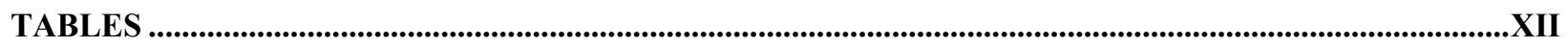

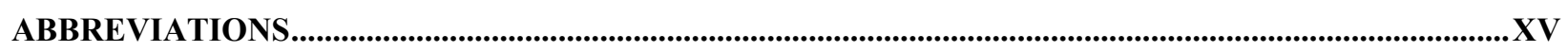

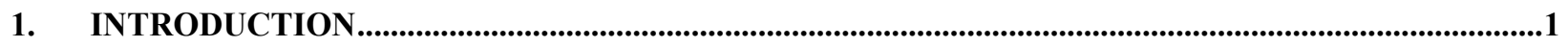

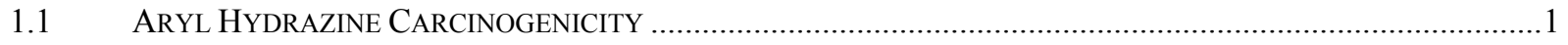

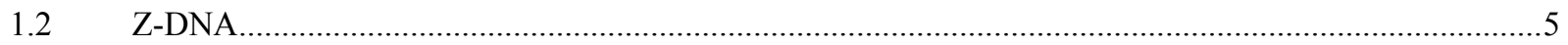

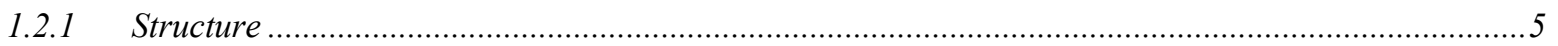

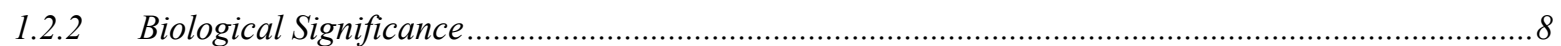

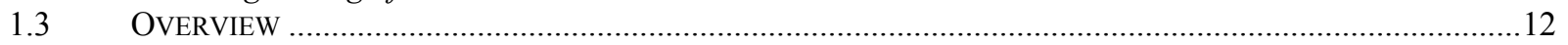

2. EXPERIMENTAL TECHNIQUES.................................................................................................................16

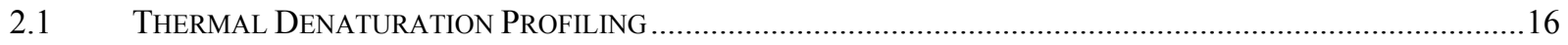

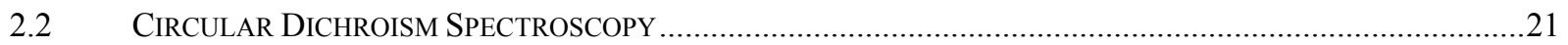

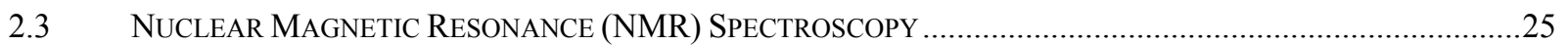

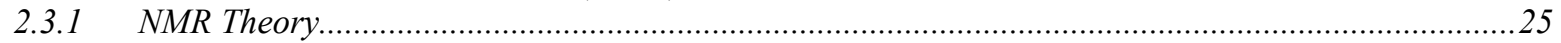

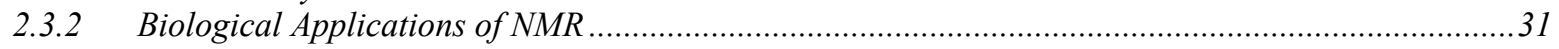

3. EXPERIMENTAL DETAILS AND RESULTS ................................................................................................36

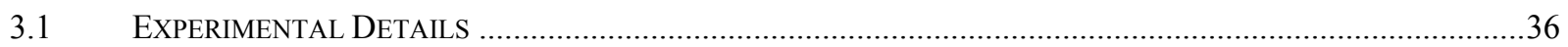

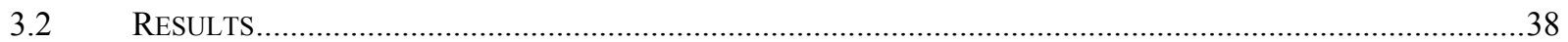

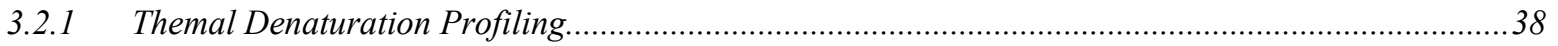

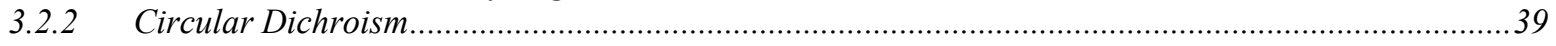

3.2.3 NMR

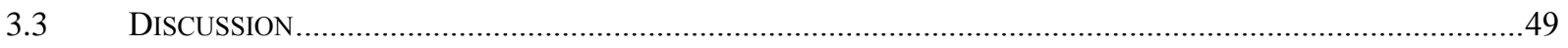

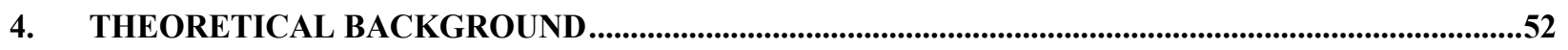

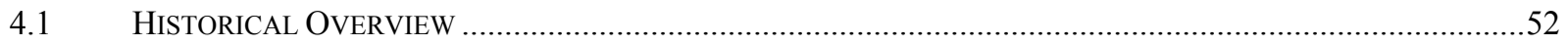

4.2 GENERAL ForCE FIELD PARAMETER DEVELOPMENT AND QUANTUM MECHANICS CALCULATIONS .........54

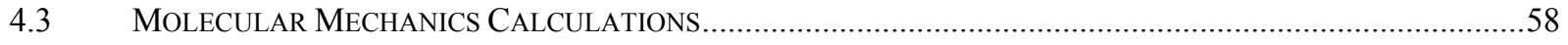

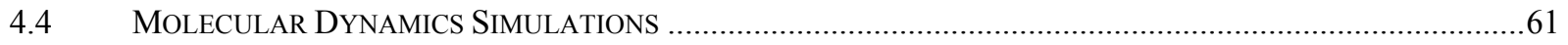

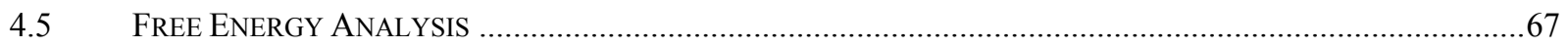


5 COMPUTATIONAL RESULTS .............................................................................................................

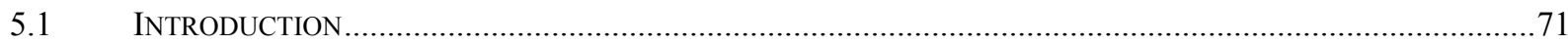

5.2 FORCE FIELD DEVELOPMENT FOR C8-TOLYL, PHENYL AND HYDROXYMETHYL-GUANINE $\ldots . . . . . . . . . . . . . . . . . .72$

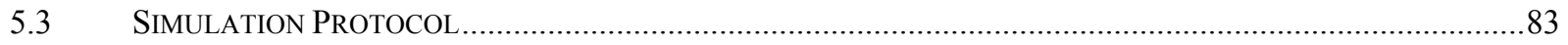

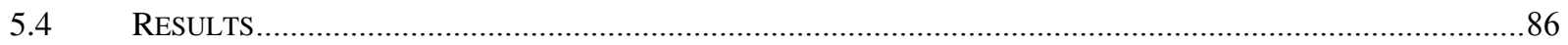

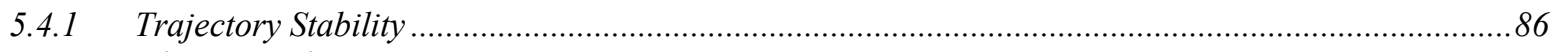

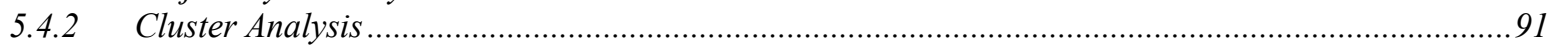

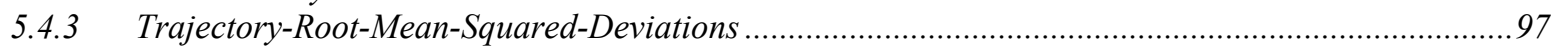

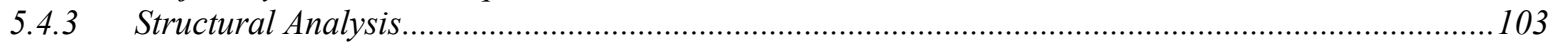

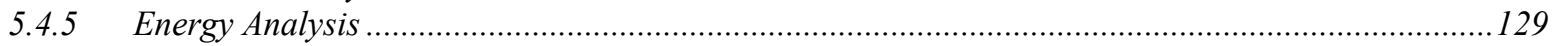

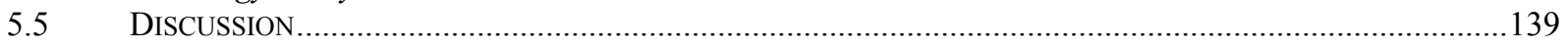

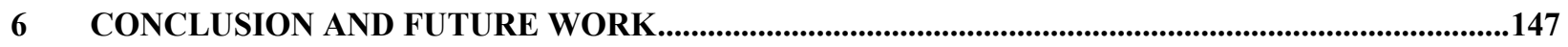

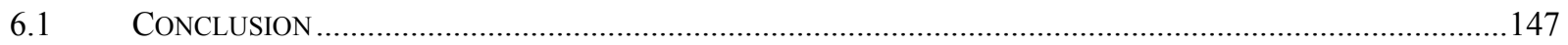

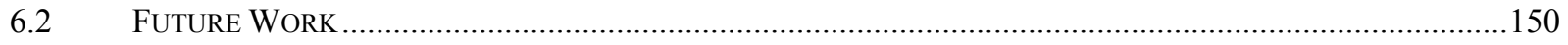

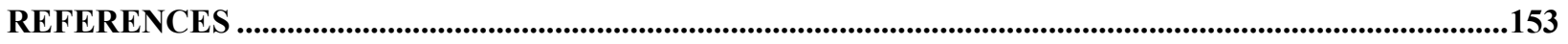

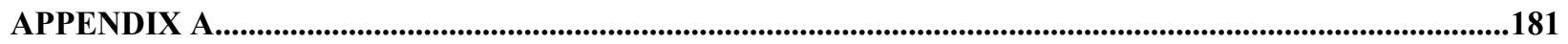

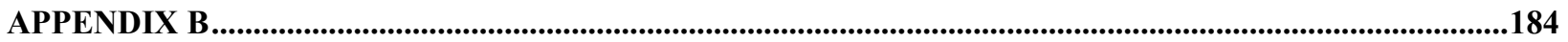

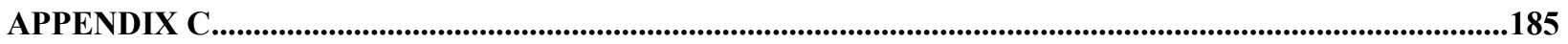




\section{Figures}

Figure 1.1. Structures of hydrazides (1), hydrazines (2), and arenediazonium ions (3) found in the mushroom Agaricus bisporus..

Figure 1.2. Metabolic conversion of aryl hydrazines to aryl radicals and C8-arylguanine adducts. 3

Figure 1.3. Structures of, from left to right, B and Z-DNA emphasizing the differences in the sugar-phosphate backbone paths of the two conformers. 5

Figure 1.4. Watson-Crick base pairing scheme for adenine (A), thymine (T), guanine (G), and cytosine $(\mathrm{C})$ bases.

Figure 1.5. Conversion of adenosine to inosine catalyzed by ADAR1. 10

Figure 1.6. Synthetic scheme for the synthesis of the phosphoramidite of C8-phenyl-2'deoxyguanosine. 15

Figure 2.1. Representation of DNA thermal denaturation/renaturation. 17

Figure 2.2. A one-step melting curve. 17

Figure 2.3. CD spectra of poly d(CG)-poly d(GC) in the A, B and Z-DNA conformations. Reprinted with permission from Riazance, J.H., Baase, W.A., Johnson Jr, W.C., Hall, K., Cruz, P., Tinoco Jr, I. Evidence for Z-form RNA by Vacuum UV Circular Dichroism. Nucleic Acids Res. (1985) 13:4983-4989.

Figure 2.4. Spin coupling between two protons............................................................ 28

Figure 2.5. Karplus Curve demonstrating the dependence of $\mathrm{J}$ on the dihedral angle, $\varphi$. 30

Figure 3.1. $\mathrm{CD}$ spectra of a) $\mathrm{CG}$ and b) $\mathrm{CG}^{8-\mathrm{Ph}}$ as a function of salt concentration. All spectra were recorded at $20^{\circ} \mathrm{C}$ in solutions containing approximately $25 \mathrm{mM}$ oligonucleotide, 10 $\mathrm{mM}$ phosphate buffer, $\mathrm{pH} 7.4$.

Figure 3.2. Aromatic and sugar regions of the NOESY spectrum of $\mathrm{CG}^{8-\mathrm{Ph}}$ showing the correlations between $\mathrm{GH} 8$ - $\mathrm{H} 1$ ' and $\mathrm{CH} 6-\mathrm{CH} 5$ (left) and the sugar protons (right). The sample was made up in $1 \mathrm{M} \mathrm{NaCl}, 10 \mathrm{mM}$ sodium phosphate buffer, $\mathrm{pH} 7.4$ at $20^{\circ} \mathrm{C}$. 
Figure 3.3. Aromatic region of the COSY spectrum of $\mathrm{CG}^{8-\mathrm{Ph}}$ showing the phenyl protons in the single-stranded (ss), B-DNA (B) and Z-DNA (Z) forms. The sample was made up in 100 $\mathrm{mM} \mathrm{NaCl}, 10 \mathrm{mM}$ sodium phosphate buffer, $\mathrm{pH} 7.4$ at $20^{\circ} \mathrm{C}$.

Figure 3.4. Numbering scheme for $\mathbf{C G}^{\mathbf{8}-\mathbf{P h}}$

Figure 4.1. Improper torsion angle I-J-K-L.

Figure 5.1. Numbering scheme for a) C8-phenylguanine, b) C8-p-tolylguanine, and c) C8-p-

hydroxymethylphenylguanine

Figure 5.2. Energy profile for rotation about the C8-p-tolylguanine bond

Figure 5.3. Numbering scheme for a) benzene, b) toluene, and c) iodobenzylalcohol.

Figure 5.4. Production run for 2 ns of the unmodified B-DNA. Total (Etot), kinetic (EKtot), and potential (EPtot) energies are in $\mathrm{kcal} / \mathrm{mole}$; density is in $\mathrm{g} / \mathrm{cm}^{3}$; pressure is in atmospheres, and volume in $\AA^{3}$.

Figure 5.5. Production run for 2 ns of the phenyl-modified B-DNA. Total (Etot), kinetic (EKtot), and potential (EPtot) energies are in $\mathrm{kcal} / \mathrm{mole}$; density is in $\mathrm{g} / \mathrm{cm}^{3}$; pressure is in atmospheres, and volume in $\AA^{3}$.

Figure 5.6. Production run for 2 ns of the tolyl-modified B-DNA. Total (Etot), kinetic (EKtot), and potential (EPtot) energies are in $\mathrm{kcal} / \mathrm{mole}$; density is in $\mathrm{g} / \mathrm{cm}^{3}$; pressure is in atmospheres, and volume in $\AA^{3}$.

Figure 5.7. Production run for 2 ns of the hydroxymethylphenyl-modified B-DNA. Total (Etot), kinetic (EKtot), and potential (EPtot) energies are in $\mathrm{kcal} / \mathrm{mole}$; density is in $\mathrm{g} / \mathrm{cm}^{3}$; pressure is in atmospheres, and volume in $\AA^{3}$.

Figure 5.8. Production run for 2 ns of the unmodified Z-DNA. Total (Etot), kinetic (EKtot), and potential (EPtot) energies are in $\mathrm{kcal} / \mathrm{mole}$; density is in $\mathrm{g} / \mathrm{cm}^{3}$; pressure is in atmospheres, and volume in $\AA^{3}$.

Figure 5.9. Production run for $2 \mathrm{~ns}$ of the phenyl-modified Z-DNA. Total (Etot), kinetic (EKtot), and potential (EPtot) energies are in $\mathrm{kcal} / \mathrm{mole}$; density is in $\mathrm{g} / \mathrm{cm} 3$; pressure is in atmospheres, and volume in $\AA^{3}$. 
Figure 5.10. Production run for 2 ns of the tolyl-modified Z-DNA. Total (Etot), kinetic (EKtot), and potential (EPtot) energies are in $\mathrm{kcal} / \mathrm{mole}$; density is in $\mathrm{g} / \mathrm{cm}^{3}$; pressure is in atmospheres, and volume in $\AA^{3}$.

Figure 5.11. Production run for 2 ns of the hydroxymethylphenyl-modified Z-DNA. Total (Etot), kinetic (EKtot), and potential (EPtot) energies are in $\mathrm{kcal} / \mathrm{mole}$; density is in $\mathrm{g} / \mathrm{cm}^{3}$; pressure is in atmospheres, and volume in $\AA^{3}$. 90

Figure 5.12. Most representative structure from the CG simulation in the B-DNA form a) side view and b) close up of (C5G6C7) and the complementary strand (G14C15G16). The base shown in bold in this and the following figures corresponds to G6.

Figure 5.13. Most representative structure from the CG simulation in the Z-DNA form a) side view and b) close up of $\mathrm{C} 5 \mathrm{G} 6 \mathrm{C} 7$ ) and the complementary strand (G14C15G16).

Figure 5.14. Most representative structure from the $\mathrm{CG}^{8-\mathrm{Ph}}$ simulation in the B-DNA form a) side view and b) close up of (C5G6C7) and the complementary strand (G14C15G16) ..... 93

Figure 5.15. Most representative structure from the $\mathrm{CG}^{8-P h}$ simulation in the Z-DNA form a) side view and b) close up of (C5G6C7) and the complementary strand (G14C15G16)..... 93

Figure 5.16. Most representative structure from the $\mathrm{CG}^{8-\mathrm{Tol}}$ simulation in the B-DNA form a) side view and b) close up of (C5G6C7) and the complementary strand (G14C15G16)..... 94

Figure 5.17. Most representative structure from the $\mathrm{CG}^{8-\mathrm{Tol}}$ simulation in the Z-DNA form a) side view and b) close up of (C5G6C7) and the complementary strand (G14C15G16)..... 94

Figure 5.18. Most representative structure from the $\mathbf{C G}^{8-\mathrm{Hmp}}$ simulation in the B-DNA form a) side view and b) close up of (C5G6C7) and the complementary strand (G14C15G16)..........95

Figure 5.19. Most representative structure from the $\mathrm{CG}^{8-\mathrm{Hmp}}$ simulation in the Z-DNA form a) side view and b) close up of (C5G6C7) and the complementary strand (G14C15G16) ..... 95

Figure 5.20. RMSD's to the most representative structures over the course of the trajectories of

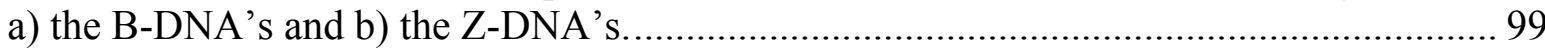

Figure 5.21. RMSD's to the starting structures over the course of the trajectories of a) the BDNA's and b) the Z-DNA's. 100 
Figure 5.22. a) RMSD's of $\mathbf{C G}, \mathbf{C G}^{8-\mathbf{P h}}, \mathbf{C G}^{\mathbf{8 - T o l}}$, and $\mathbf{C G}^{\mathbf{8 - H m p}}$ in the B-DNA conformation to canonical A-DNA, b) RMSD's of $\mathbf{C G}, \mathbf{C G}^{\mathbf{8 - P h}}, \mathbf{C G}^{\mathbf{8 - T o l}}$, and $\mathbf{C G}^{\mathbf{8 - H m p}}$ in the B-DNA conformation to canonical B-DNA, and c) RMSDs of $\mathbf{C G}^{\mathbf{8 - P h},} \mathbf{C G}^{\mathbf{8 - T o l}}$, and $\mathbf{C G}^{\mathbf{8 - H m p}}$ in the B-DNA conformation to the most representative structure of the unmodified BDNA

Figure 5.23. a) RMSD's of $\mathbf{C G}, \mathbf{C G}^{\mathbf{8 - P h}}, \mathbf{C G}^{\mathbf{8 - T o l}}$, and $\mathbf{C G}^{\mathbf{8 - H m p}}$ in the Z-DNA conformation to canonical Z-DNA, and b) RMSD's of $\mathbf{C G}, \mathbf{C G}^{\mathbf{8 - P h}}, \mathbf{C G}^{\mathbf{8 - T o l}}$, and $\mathbf{C G}^{\mathbf{8 - H m p}}$ in the Z-DNA conformation to the most representative structure of the unmodified Z-DNA. 102

Figure 5.24. Definition of a) torsional angles and b) the helical axis system. ${ }^{141}$ 104

Figure 5.25. Average diameter for unmodified $(\bullet)$, phenyl $(\boldsymbol{\Delta})$, tolyl (घ), and hydroxymethylphenyl ( $\bullet$ modified a) B and b) Z-DNA’s. 108

Figure 5.26. Torsion angles for the most representative B-DNA structures. Values are for bases 1-20 with each grid representing five bases each. The values near 1, 10, 11, and 20 may be weird due to end effects.

Figure 5.27. Torsion angles for the most representative Z-DNA structures. Values are for bases 1-20 with each grid representing five bases each. The values near 1, 10, 11, and 20 may be weird due to end effects.

Figure 5.28. Diagrams illustrating movements in the positive direction of base pairs or successive bases. $^{141}$

Figure 5.29. Shift ( -2 to $2 \AA)$, slide ( -2 to $2 \AA)$, rise $(0$ to $7 \AA)$, tilt( $\left(-22\right.$ to $\left.22^{\circ}\right)$, roll $\left(-24\right.$ to $\left.30^{\circ}\right)$, and twist $\left(-3\right.$ to $\left.51^{\circ}\right)$ of base pairs over the course of the 2 ns production trajectory for a) $\mathbf{C G}$, b) $\mathbf{C G}^{\mathbf{8 - P h}}$, c) $\mathbf{C G}^{\mathbf{8 - T o l}}$, and d) $\mathbf{C G}^{\mathbf{8 - H m p}}$ in the B-DNA conformation. Ranges, given in parentheses after each parameter, are identical in a-d.

Figure 5.30. X-displacement (-4 to $2 \AA$ ), y-displacement (-3 to $2 \AA)$, inclination ( -26 to $30^{\circ}$ ), and tip $\left(-27\right.$ to $\left.31^{\circ}\right)$ of base pairs over the course of the 2 ns production trajectory for a) $\mathbf{C G}$, b) $\mathbf{C G}^{\mathbf{8 - P h}}$, c) $\mathbf{C G}^{\mathbf{8 - T o l}}$, and d) $\mathbf{C G}^{\mathbf{8 - H m p}}$ in the B-DNA conformation. Ranges, given in parentheses after each parameter, are identical in a-d.

Figure 5.31. Shear ( -2 to $1 \AA$ ), stretch ( -2 to $2 \AA$ ), stagger (-4 to $1 \AA$ ), buckle (-56 to $56^{\circ}$ ), propeller $\left(-59\right.$ to $\left.32^{\circ}\right)$, and opening $\left(-12\right.$ to $\left.28^{\circ}\right)$ of base pairs over the course of the $2 \mathrm{~ns}$ production trajectory for a) $\mathbf{C G}$, b) $\mathbf{C G}^{\mathbf{8 - P h}}$, c) $\mathbf{C G}^{\mathbf{8 - \mathbf { T } o l}}$, and d) $\mathbf{C G}^{\mathbf{8 - H m p}}$ in the B-DNA conformation. Ranges, given in parentheses after each parameter, are identical in a-d.... 119 
Figure 5.32. Distance between the hydrogen of the hydroxy moiety of HMP in G6 and G16 and the bases' own $02 \mathrm{P}$ oxygen over the course of the 2 ns production run. 118

Figure 5.33. Shift (-1 to 2 ), slide ( -10 to $2 \AA$ ), rise (-10 to $1 \AA$ ), tilt (-16 to $\left.17^{\circ}\right)$, roll (-68 to $\left.48^{\circ}\right)$, and twist $\left(-32\right.$ to $\left.81^{\circ}\right)$ of base pairs over the course of the 2 ns production trajectory for a) $\mathbf{C G}$, b) $\mathbf{C G}^{\mathbf{8 - P h}}$, c) $\mathbf{C G}^{\mathbf{8 - T o l}}$, and d) $\mathbf{C G}^{\mathbf{8 - H m p}}$ in the Z-DNA conformation. Ranges, given in parentheses after each parameter, are identical in a-d.

Figure 5.34. X-displacement (-8 to $4 \AA$ ), y-displacement (-3 to $6 \AA$ ), inclination ( -85 to $\left.19^{\circ}\right)$, and tip $\left(-22\right.$ to $\left.24^{\circ}\right)$ of base pairs over the course of the 2 ns production trajectory for a) CG, b) $\mathbf{C G}^{\text {8-Ph }}$, c) $\mathbf{C G}^{\text {8-Tol }}$, and d) $\mathbf{C G}^{\text {8-Hmp }}$ in the Z-DNA conformation. Ranges, given in parentheses after each parameter, are identical in a-d.

Figure 5.35. Shear (-1 to $1 \AA$ ), stretch (-3 to $5 \AA$ ), stagger (-1 to $1 \AA$ ), buckle (-60 to $\left.30^{\circ}\right)$, propeller $\left(-30\right.$ to $\left.44^{\circ}\right)$, and opening $\left(-25\right.$ to $\left.26^{\circ}\right)$ of base pairs over the course of the $2 \mathrm{~ns}$ production trajectory for a) $\mathbf{C G}$, b) $\mathbf{C G}^{\text {8-Ph }}$, c) $\mathbf{C G}^{\text {-Tol }}$, and d) $\mathbf{C G}^{\text {8-Hmp }}$ in the Z-DNA conformation. Ranges, given in parentheses after each parameter, are identical in a-d. ... 123

Figure 5.36. Structures of the $\mathrm{C} 2$ '-endo and $\mathrm{C} 3$ '-endo preferred sugar puckers. 125

Figure 5.37. Conformation plots of $\chi$ as a function of $\delta$ for a) the B-DNA's and b) the Z-DNA's. The boxed A indicates the classical A helix region, and the line indicates the broad distribution of conformers found in B-DNA as established by fiber diffraction. ${ }^{146}$ 126

Figure 5.38. Helical parameters calculated by CURVES for the unmodified and modified BDNA's. Parameters for A-DNA and B-DNA fibers are indicated by the boxed A's and B's, respectively. ${ }^{148}$ Inclination, roll, and twist are in units of degrees. 128

Figure 5.39. Total (Etot) energy comparison for all production runs. Energy is in units of $\mathrm{kcal} / \mathrm{mole}$.

Figure 5.40. Linear relationship between the number of solvent residues and average total energy calculated during the production MD simulation.

Figure 5.41. Close-up of, from bottom to top, the fourth through eighth base pair steps of the unmodified B-DNA

Figure 5.42. Close-up of, from bottom to top, the fourth through eighth base pair steps of the phenyl-modified B-DNA.

Figure 5.43. Close-up of, from bottom to top, the fourth through eighth base pair steps of the tolyl-modified B-DNA. 
Figure 5.44. Close up of, from bottom to top, the fourth through eighth base pair steps of the hydroxymethylphenyl-modified B-DNA................................................................. 142 


\section{Tables}

Table 2.1. Melting temperatures in units of degrees Celsius for various G8-modified bases paired with the four natural bases and incorporated into duplex oligonucleotides.

Table 2.2. Pascal's triangle.

Table 2.3. Typical resonance assignments for base/sugar protons, highlighting the differences between $\mathrm{B}$ and Z-DNA in bold print. ${ }^{66}$

Table 2.4. Approximate coupling constants ${ }^{61}$ and corresponding dihedral angles ${ }^{85}$ for C 3 '-endo

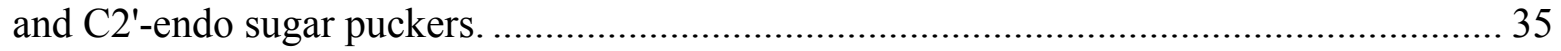

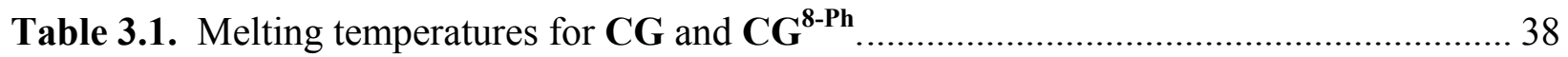

Table 3.2. Chemical Shifts of CG in the B-DNA Conformation given in units of ppm. .......... 42

Table 3.3. Chemical Shifts of $\mathbf{C G}^{\mathbf{8 - P h}}$ in the Z-DNA Conformation given in units of ppm...... 43

Table 3.4. Single-stranded, B, and Z-DNA chemical shifts of the C8-phenyl protons of $\mathbf{C G}^{\mathbf{8}-\mathbf{P h}}$

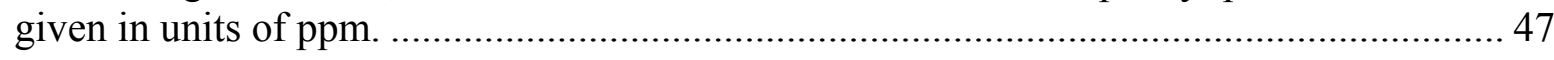

Table 5.1. Cornell 95 force field parameters for bonds in C8-arylguanines for use with Amber. Parameters shown in bold type are those developed in this work for the Cornell 95 force field. Parameters shown in parentheses are from crystal structures.

Table 5.2. Cornell 95 force field parameters for bond angles in C8-arylguanines for use with Amber. Parameters shown in parentheses are from crystal structures.

Table 5.3. Cornell 95 force field parameters for bond torsions in C8-arylguanines for use with Amber. Parameters shown in parentheses are from crystal structures.

Table 5.4. Cornell 95 force field parameters for improper bond torsions in $\mathrm{C} 8$-arylguanines for use with Amber.

Table 5.5. Partial electronic charges of C8-arylguanines evaluated by fitting the HF6-31G* electrostatic potentials with the RESP algorithm.....

Table 5.6. Box dimensions and number of solvent residues for each system. 
Table 5.7. Average torsion angles (in degrees) from crystal structures ${ }^{140}$ and as calculated by CURVES for canonical structures in parentheses. 103

Table 5.8a. Global groove width and depth parameters for unmodified and modified duplexes in the B-DNA conformation. 106

Table 5.8b. Global groove width and depth parameters for unmodified and modified duplexes in the Z-DNA conformation. 107

Table 5.9a. Diameter of unmodified and modified duplexes in the B-DNA conformation. .... 108

Table 5.9b. Diameter of unmodified and modified duplexes in the Z-DNA conformation. .... 108

Table 5.10a. Global axis parameters for unmodified and modified duplexes in the B-DNA conformation.

Table 5.10b. Global axis parameters for unmodified and modified duplexes in the Z-DNA conformation.

Table 5.11. Helicoidal parameter names and definitions for base pair values. ${ }^{143}$ 114

Table 5.12. Ranges of the sugar pucker phase angle for all conformations of the ribose; * indicates sugar puckers found for guanines in Z-DNA and **indicates puckers found in BDNA. $^{146}$

Table 5.13. Relative order of total and free energies for the modified and unmodified DNA's in the $\mathrm{B}$ and $\mathrm{Z}$ conformations from the total energy calculated during the MD simulation, and the PB and GB solvent models. The structures are listed in order from highest to lowest energy. 134

Table 5.14. Average energies (in $\mathrm{kcal} / \mathrm{mol}$ and rounded to four significant digits) and standard errors of the mean energies (in parentheses) from 145 equally spaced configurations sampled from frames 500-1950 of the B-DNA trajectories calculated as discussed above. Numbers in parentheses represent the standard error of the mean, i.e., the mean divided by the square root of the number of snapshots sampled.

Table 5.15. Average energies (in $\mathrm{kcal} / \mathrm{mol}$ and rounded to four significant digits) and standard errors of the mean energies (in parentheses) from 145 equally spaced configurations sampled from frames 500-1950 of the Z-DNA trajectories calculated as discussed above. Numbers in parentheses represent the standard error of the mean, i.e., the mean divided by the square root of the number of snapshots sampled. 
Table 5.16. Average energies (in $\mathrm{kcal} / \mathrm{mol}$ and rounded to four significant digits) from 145 equally spaced configurations sampled from frames 500-1950 of the B and Z-DNA trajectories calculated as discussed above varying salt concentration for the PB

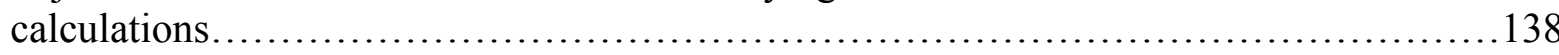

Table 5.17. Comparison of selected components of the free energy calculations for the unmodified B-DNA to those values found in the literature. 143 


\begin{tabular}{|c|c|c|}
\hline \multicolumn{3}{|c|}{ Abbreviations } \\
\hline 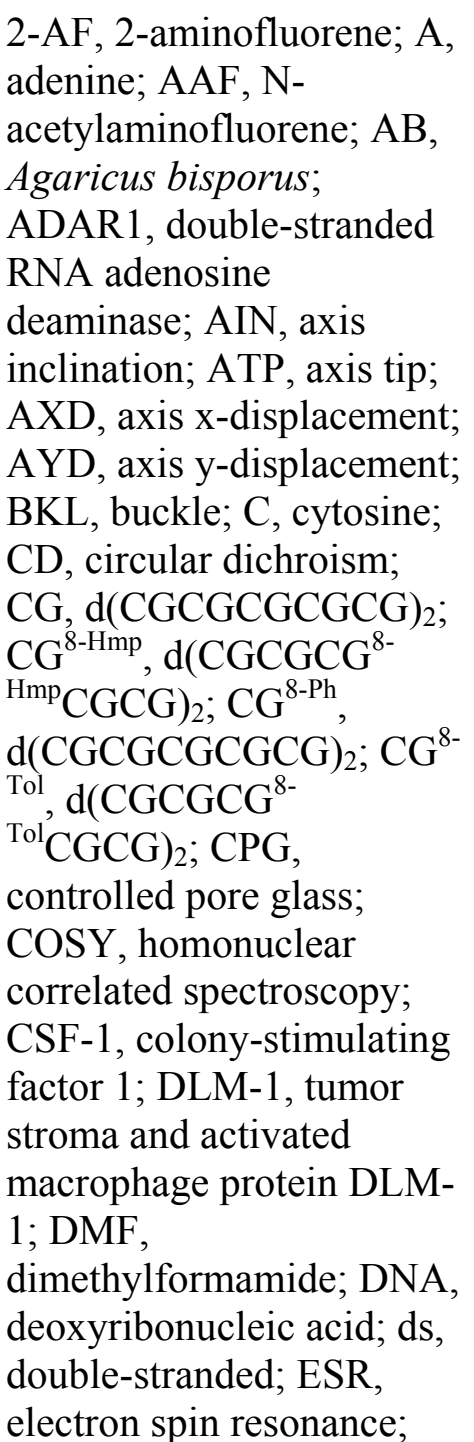 & $\begin{array}{l}\Delta \mathrm{G}, \text { free energy; G, } \\
\text { guanine; GB, generalized } \\
\text { Born; }{ }^{1} \mathrm{H}, \text { one-dimentional } \\
\text { proton; } \Delta \mathrm{H} \text {, enthalpy; HF, } \\
\text { hartree flock; HMP, } \\
\text { hydroxymethylphenyl; } \\
\text { INC, inclination; IR, } \\
\text { infrared radiation; LAH, } \\
\text { lithium aluminum hydride; } \\
\mathrm{C}^{5-M e}, 5 \text {-methyl-2'- } \\
\text { deoxycytosine; MD, } \\
\text { molecular dynamics; MM, } \\
\text { molecular mechanics; } \\
\text { MM-PBSA, molecular } \\
\text { mechanics Poisson- } \\
\text { Bolzmann surface area; } \\
\text { MS, mass spectra; PB, } \\
\text { Poisson-Boltzman; } N c l, \\
\text { nucleolin; NMR, nuclear } \\
\text { magnetic resonance; nOe, } \\
\text { nuclear Overhauser effect, } \\
\text { NOESY, nOe correlated } \\
\text { spectroscopy; 8-oxodG, 8- } \\
\text { oxo-2'de-oxyguanosine; } \\
\text { oligo, oligonucleotide; } \\
\text { OPN, opening; PAH, } \\
\text { polycyclic aromatic } \\
\text { hydrocarbon; PME, } \\
\text { particle mesh Ewald; PRP, } \\
\text { propeller; PU, purine; PY, }\end{array}$ & $\begin{array}{l}\text { pyrimidine; QM, quantum } \\
\text { mechanics; RESP, } \\
\text { restrained electrostatic } \\
\text { potential; RIS, rise; } \\
\text { RMSD, root mean squared } \\
\text { deviation; ROESY, } \\
\text { rotating frame Overhauser } \\
\text { spectroscopy; ROL, roll; } \\
\text { ROS, reactive oxidative } \\
\text { species; RNA, ribonucleic } \\
\text { acid; } \Delta S, \text { entropy; SCF, } \\
\text { self consistent field; SHF, } \\
\text { shift; SHR, shear; } \\
\text { SLD,slide; ss, single- } \\
\text { stranded; STG, stagger; } \\
\text { STR, stretch; T, thymine; } \\
\text { TEA, triethylamine; THF, } \\
\text { tetrahydofuran; TIP, tip; } \\
\text { TLT, tilt; Tm, thermal } \\
\text { denaturation melting } \\
\text { temperature; TMS, } \\
\text { tetramethylsilane; tris, (m- } \\
\text { sulfonatephenyl)phosphate } \\
\text { trisodium; TWT, twist; } \\
\text { UV, ultraviolet } \\
\text { spectroscopy; XDP, x- } \\
\text { displacement; YDP, y- } \\
\text { displacement }\end{array}$ \\
\hline
\end{tabular}




\section{CHAPTER 1}

\section{Introduction}

\subsection{Aryl Hydrazine Carcinogenicity}

Aryl hydrazines come from a variety of sources including industrial, pharmaceutical, and natural origins. We have been studying aryl hydrazines that are present in the mushroom Agaricus bisporus (AB), the type typically found in grocery stores, salad bars, etc. AB contains the hydrazide known as Agaritine and several related hydrazines, hydrazides, and arenediazonium ions as shown in Figure 1.1. All of these compounds, except Agaritine (1), have been shown to be carcinogenic and produce, primarily, blood vessel and lung tumors. ${ }^{1}$
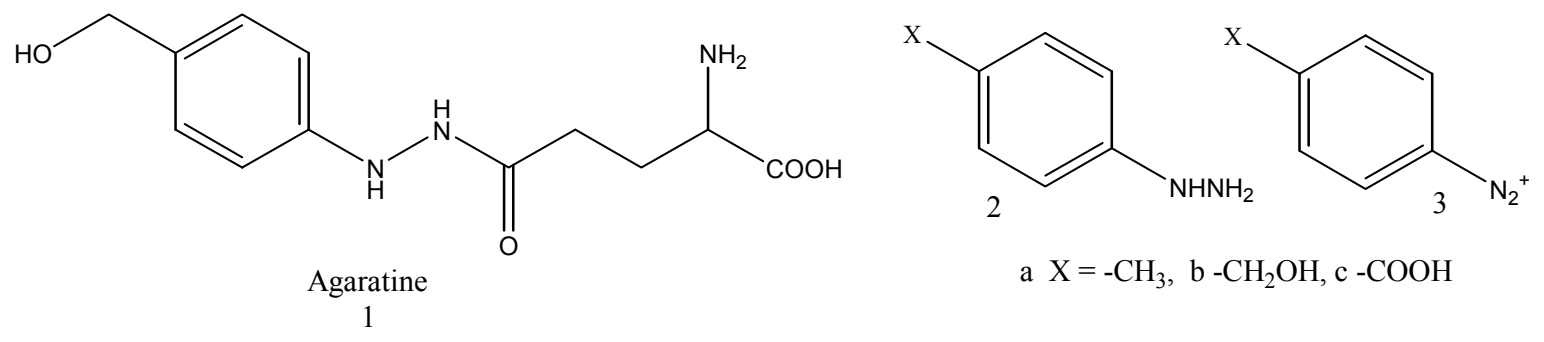

Figure 1.1. Structures of hydrazides (1), hydrazines (2), and arenediazonium ions (3) found in the mushroom Agaricus bisporus.

It has been shown that aryl hydrazines and arenediazonium ions, like those found in $\mathrm{AB}$ are metabolized to aryl radicals in vitro and in cells. ${ }^{2}$ These radicals can then damage deoxyribonucleic acid (DNA) by several possible routes including depurination, reactive oxidative species (ROS) formation, and adduct formation. A significant amount of depurination occurs during the reaction of DNA with aryl radicals, and the bases that are cleaved have been found to be C8-arylpurines. ${ }^{3}$ It should be noted that studies involving the reaction of diazoquinones with purines and pyrimidines failed to isolate products from the later 
nucleosides. $^{4}$ When depurination occurs, DNA replication opposite the abasic site often leads to the insertion of adenine. Subsequent replication establishes the mutation as a $\mathrm{G} \rightarrow \mathrm{T}$ transversion if the depurinated base is guanine. It is also possible that the enzymatic oxidation of aryl radicals may result in the formation of ROS, causing oxidative damage to DNA and ultimately misreading of the damaged bases. ${ }^{5}$ Oxidatively damaged guanine is misread as thymidine, ${ }^{6}$ once again subsequent replication establishes the mutation as a $\mathrm{G} \rightarrow \mathrm{T}$ transversion as for the case of guanine depurination. The third possible outcome of the reaction of aryl radicals and DNA is formation of C8-arylpurines.

There are two mechanisms for C8-arylpurine formation: direct radical attack from which C8-arylguanine is the predominant product as shown in Figure 1.2 and N6-triazene adduct decomposition resulting in the formation of $\mathrm{C} 8$-aryladenine. The reaction of carbon centered radicals with DNA are generally more selective than reactions with oxygen centered radicals, and the resulting products are mainly C8-arylguanine adducts. ${ }^{7,8,9,10}$ N6-triazene adduct formation is the result of the reaction of DNA with arenediazonium ions, the precursors to aryl radicals, ${ }^{11}$ and will not be discussed further in this work. C8-arylguanine carcinogenicity may result from their influence on base pairing properties, leading to apurinic site formation or misreading and ultimately a $\mathrm{G} \rightarrow \mathrm{T}$ transversion as in the previous examples. It is also possible that the mutagenic properties of $\mathrm{C} 8$-arylguanines result from a conformational change in the DNA upon adduct formation. C8-guanine modification is known to stabilize the syn glycosidic bond conformation due to an unfavorable steric interaction between groups in the C8-guanine position and the $\mathrm{H}-2$ " proton. ${ }^{12}$ 


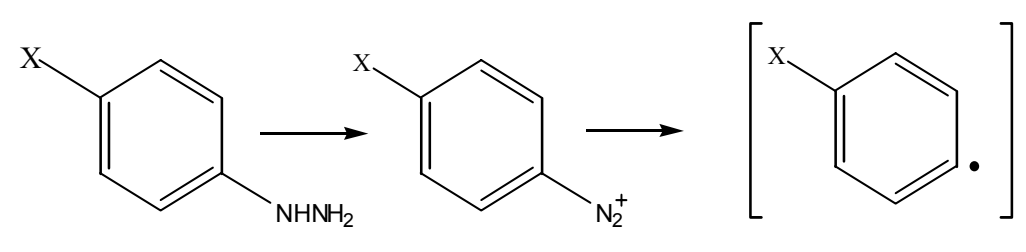

Agaricus bisporus

4

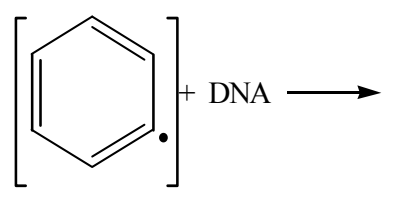

Arenediazonium Ion 5

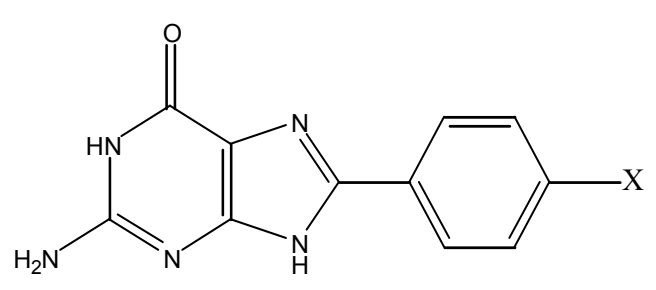

$\mathrm{C}^{8-}$ Arylguanine

6

Figure 1.2. Metabolic conversion of aryl hydrazines to aryl radicals and C8-arylguanine adducts.

The formation of C8-arylguanine adducts and their relationship to mutagenicity are poorly understood. Depurination, ROS formation, and adduct formation resulting in misreading or conformational changes are all possible routes in the metabolism of aryl radicals and their ultimate carcinogenicity. Polycyclic aromatic hydrocarbon (PAH) guanine adducts cause misreading ${ }^{13}$ and depurination. ${ }^{14} \mathrm{~N}$-acetylaminofluorine (AAF) forms $\mathrm{C} 8$-guanine adducts that intercalate into the helix and are also misread. ${ }^{15}$ However, the miscoding properties of 8phenylguanine and its effect on stability have been reported. ${ }^{16}$ The low levels of mutations in daughter strands found in these studies suggest that the correlation between C8-arylguanine adduct formation and carcinogenicity ${ }^{11}$ is not the result of misreading or apurinic site formation. Furthermore, many $\mathrm{C} 8$-adducts that are misread have a hetero atom attached to $\mathrm{C} 8$ that can participate in hydrogen bonding and base pair formation. This is not the case with C8-arylgunine adducts and it is not obvious how the lack of heteroatom at the $\mathrm{C} 8$ position might alter base pairing properties. ROS must also be considered in developing a complete picture of the 
mechanism of aryl hydrazine carcinogenicity. To this end, studies have been performed using various methods including free radical scavengers, antioxidants, and electron spin resonance (ESR). The overall conclusion has been that in cells the predominant radical species present is the aryl and not ROS such as hydroxy or superoxide; ${ }^{17}$ therefore, it is likely that the aryl radical is of the most significance from a physiological standpoint.

While the above mechanisms cannot be completely ruled out, the formation of C8adducts is known to have a profound effect on the local conformation of DNA, which could in turn be responsible for the mutagenic and carcinogenic properties of aryl hydrazines. In a study of C8-methylguanine, formed from methyl hydrazine, it was shown that the B-DNA form of an alternating purine-pyrimidine sequence was destabilized. At sodium chloride concentrations above $5 \mathrm{mM}$, the Z-DNA form predominates. ${ }^{18}$ All known C8-guanine adducts, as well as AAF adducts generally prefer to adopt a syn conformation about the glycosidic bond as found for guanines in Z-DNA, rather than the normal anti conformation typical of B-DNA. These observations suggested to us the possibility that the C8-arylguanine adducts that we have observed $^{11,19}$ may destabilize B-DNA and stabilize Z-DNA. In light of the identification of sequences prone to Z-DNA formation in oncogenes such as $\mathrm{c}-\mathrm{Myc}^{20,21}$ and recent research that has shown that Z-DNA may regulate gene expression and cell transformation, ${ }^{22}$ we propose that C8-arylguanine adducts formed from aryl hydrazines may activate these genes by inducing such a conformation shift. 


\subsection{Z-DNA}

\subsubsection{Structure}

DNA exists in multiple conformations, aside from the familiar right-handed B-DNA double helix as illustrated in Figure 1.3. Z-DNA is one such conformer, and unlike B-DNA it is left-handed. ${ }^{23}$ As is the case for B-DNA, double-stranded Z-DNA is antiparallel, and WatsonCrick base pairing, shown in Figure 1.4, joins the two strands. The name Z-DNA was coined from the characteristic zigzag path of the phosphate sugar backbone induced by the alternating anti and syn-conformations of the bases about the N-glycosidic bond. Sequences with alternating pyrimidine/purine bases convert most readily from the B to Z-DNA conformation, especially alternating deoxycytidine-deoxyguanosine residues. ${ }^{24,25}$ The broad, concave major groove of B-DNA is converted into the outer convex surface of Z-DNA on which (normally less inaccessible parts of the bases) cytosine-C5 and guanine-N7 and C8 are exposed. The minor groove of Z-DNA is so deep it contains the helix axis.
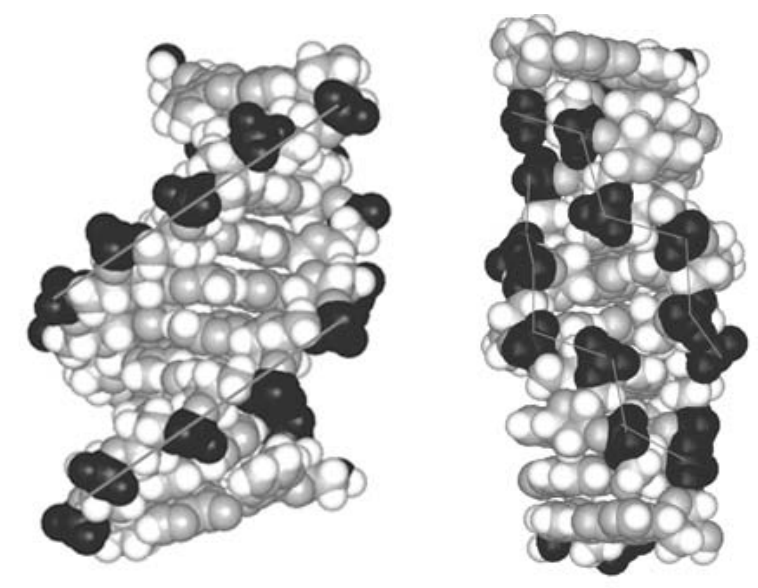

Figure 1.3. Structures of, from left to right, $B$ and Z-DNA emphasizing the differences in the sugar-phosphate backbone paths of the two conformers. 


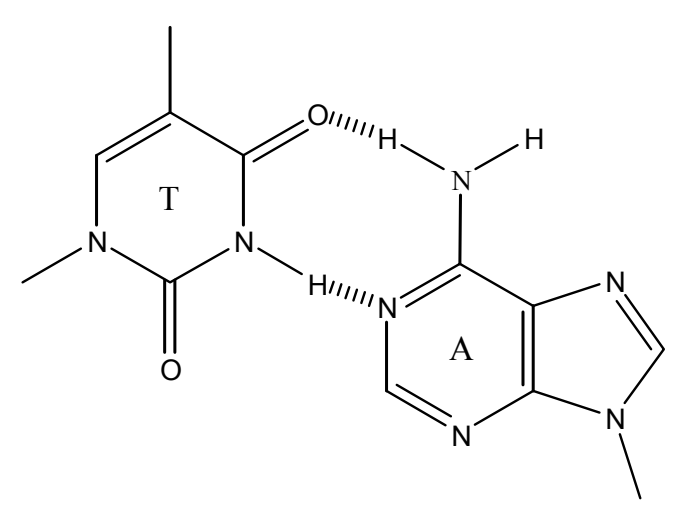

$\mathrm{T}: \mathrm{A}$

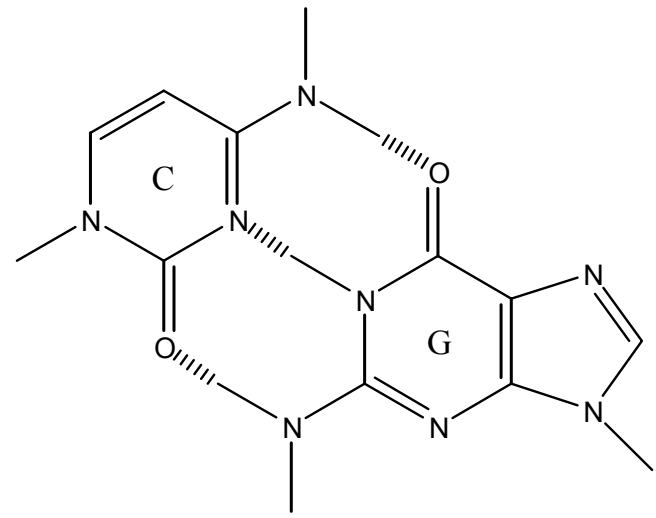

$\mathrm{C}: \mathrm{G}$

Figure 1.4. Watson-Crick base pairing scheme for adenine (A), thymine (T), guanine (G), and cytosine $(\mathrm{C})$ bases.

There are a number of factors known to affect the relative stability of B and Z-DNA including sequence effects, salt concentration, counterion identity, base modifications, hydrophobicity, and temperature. All of these effects are important and, to some extent, overlap with one another. Z-DNA formation is directly correlated to base content of the sequence. $\mathrm{d}(\mathrm{CG})_{\mathrm{n}}$ is best, followed by $\mathrm{d}(\mathrm{TG})_{\mathrm{n}}, \mathrm{d}(\mathrm{GGGC})_{\mathrm{n}}$, and $\mathrm{d}(\mathrm{TA})_{\mathrm{n}} \cdot{ }^{26}$ Out of alteration base pairs can be accommodated under conditions very favorable for formation of Z-DNA. Conditions that promote Z-DNA formation include high salt concentration and the presence of divalent cations such as polyamines. These positively charged molecules decrease the electrostatic repulsion of closely spaced (relative to B-DNA) phosphate residues. Hydrophobic solutions such as ethanol ${ }^{27}$ also have a stabilizing effect of Z-DNA. In B-DNA the bases face in toward the center of the helix, but in the $\mathrm{Z}$ form, these hydrophobic residues face out into the solvent. Base modifications including cytosine methylation and all known guanine C8 modifications also contribute to the stability of Z-DNA. The guanine modifications change the conformation about 
the glycosidic bond, stabilizing the syn conformation as found in Z-DNA. Studies have also shown that low temperature ${ }^{18,28}$ or high pressure ${ }^{29}$ push the $\mathrm{B}-\mathrm{Z}$ equilibrium in the $\mathrm{Z}$ direction. Z-DNA is also stabilized by negative supercoiling, ${ }^{30}$ which arises behind RNA polymerase during transcription.

The first evidence suggesting the existence of Z-DNA came from optical studies of poly $\mathrm{d}(\mathrm{CG})_{\mathrm{n}} \cdot{ }^{31}$ Changes were observed in the circular dichroism (CD) spectrum between 230 and 300 $\mathrm{nm}$ for low and high-salt solutions. ${ }^{31}$ In $3 \mathrm{M} \mathrm{NaCl}$, the $\mathrm{CD}$ spectrum in the 280-300 $\mathrm{nm}$ region, normally positive for A and B-DNA, is approximately inverted compared to the B-form spectrum observed in low-salt solution. An atomic resolution crystallographic study of $\mathrm{d}(\mathrm{GC})_{3}$ revealed the existence of a left-handed double helix, ${ }^{23}$ unraveling the physical reason for the negative ellipticity observed in the optical studies. ${ }^{31}$ 


\subsubsection{Biological Significance}

The formation of Z-DNA in vivo has been demonstrated in prokaryotes $;^{32,33,34,35}$ however, direct evidence demonstrating the existence of Z-DNA in eukaryotic systems has been difficult to find. A characteristic specific to $\mathrm{Z}$ and not B-DNA, its immunogenicity, ${ }^{36}$ led to the first suggestion that Z-DNA was found in eukaryotic systems. Analysis of sera obtained from patients with the autoimmune disease lupus erythematosis showed that these sufferers produced highly specific antibodies for Z-DNA. ${ }^{37}$ The degree of specificity implied that Z-DNA is the cognate antigen and, by association, the existence of Z-DNA in vivo. Further experimentation has provided more direct evidence for the formation of Z-DNA in eukaryotics. ${ }^{20,21,38,39}$ These studies suggest that Z-DNA forms behind an active RNA polymerase, and negative supercoiling generated by transcription stabilizes it.

Despite the distinctive zigzag of its sugar-phosphate backbone, deriving a functional role in biological processes for Z-DNA is an ongoing challenge. Recent studies have found that sequences prone to Z-DNA formation such as TG and GC repeats are required for the activation of the gene promoter human colony-stimulating factor $1(\mathrm{CSF}-1){ }^{22}$ These findings suggest a functional role for Z-DNA in gene activation events. Z-DNA has also been implicated in negative modulation of the rat nucleolin $(\mathrm{Ncl})$ gene promoter. ${ }^{40}$ The purine-pyrimidine dinucleotide repeat in $\mathrm{Ncl}$ is highly conserved between several rodent species, ${ }^{41}$ furthering supporting a biologically functional role for Z-DNA.

5-methylcytosine lends further biological relevance to the role of Z-DNA. The vast majority of DNA nucleotides exist in the typical A, T, G, C forms depicted in standard texts. An exception to this in humans (and other eukaryotes) is the occurrence of 5-methylcytosine. 
Methylation of cytosines occurs only within double-stranded (ds) DNA. In addition, 5-methyl cytosines are always located immediately 5 ' of a guanine residue, in so-called $\mathrm{CpG}$ sequences. About $60 \%$ of the cytosines in human $\mathrm{CpG}$ sequences are methylated. Once a cytosine located in a specific gene region has become methylated, it is usually copied after each successive round of DNA replication by enzymes called DNA methylases. Methylation plays very important roles in DNA replication/repair mechanisms, as well as in regulating gene expression. ${ }^{42}$ DNA regions with extensive 5-methylcytosine content favor the Z-DNA conformation.

Characterization of Z-DNA binding proteins may ultimately provide the key to unlocking the elusive biological role of Z-DNA. ADAR1, double-stranded RNA adenosine deaminase, is one such protein. ${ }^{43}$ Two Z-DNA binding motifs, Z $\alpha$ and Z $\beta$, were identified in ADAR1 by mapping studies. ${ }^{44}$ As shown in Figure 1.5, ADAR1 catalyzes the deamination of adenosine to form inosine in double-stranded mRNA, which is translated as guanosine. ${ }^{45}$ The consequence of this editing process can result in the placement of an amino acid other than that encoded by a particular gene. In this manner, ADAR1 acts to regulate gene expression by allowing multiple proteins to be synthesized from a single encoded sequence. Unfortunately, the Z-DNA binding motifs of ADAR1 appear to be conformer and not sequence specific. ${ }^{46}$ If the motifs were sequence specific, the identification of genes edited by ADAR1 and thus the quest for the biological function of Z-DNA would be simplified. 


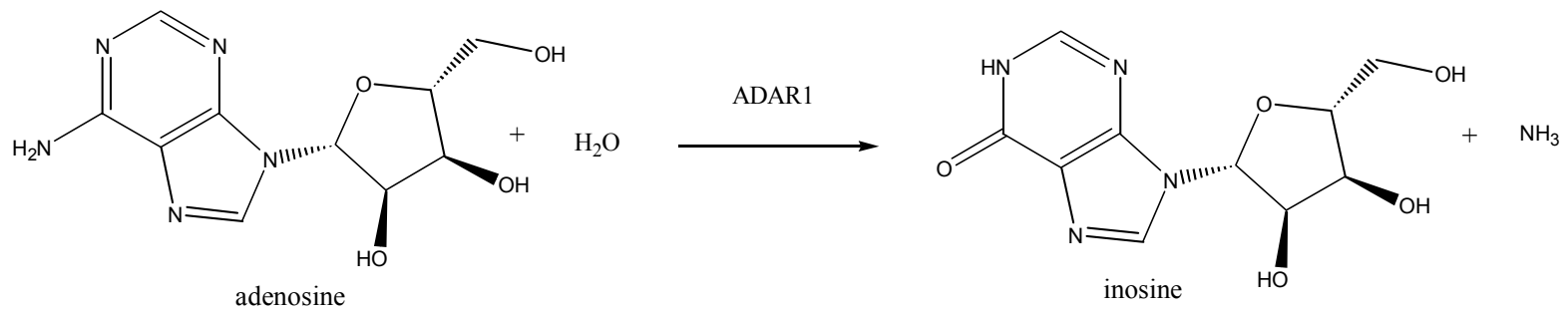

Figure 1.5. Conversion of adenosine to inosine catalyzed by ADAR1.

The co-crystallization of the Z $\alpha$ domain of ADAR1 bound to Z-DNA ${ }^{47}$ provides further evidence in support of the conformation specific binding of $Z \alpha$. The contacts of the complex were found to be primarily along the sugar-phosphate backbone, but a single base contact with H8 of a guanine base (in the syn conformation) was also observed. These interactions are highly specific to Z-DNA. From the crystal structure, the residues essential to Z-DNA recognition were used to search for other proteins with Z-DNA binding motifs. The tumor-associated protein, DLM-1, was found to contain a domain similar to Z $\alpha$ of ADAR $1{ }^{48}$ It was subsequently cocrystallized bound to Z-DNA, and a structure-specific recognition sequence similar to ADAR1's was revealed. The $Z \alpha_{\text {DLM }}-Z-D N A$ complex was found to be similar to that of $Z \alpha_{A D A R 1}-Z-D N A$. The sequence of $Z \alpha_{\text {DLM }}$ is about $35 \%$ homologous to that of $Z \alpha_{A D A R 1}$, and those residues critical for maintaining the domain fold and contacting DNA are conserved. ADAR1 and DLM-1 are both inducible by $\gamma$-interferon, suggesting they, and by association Z-DNA, may have significance in the host-response to pathogens. ${ }^{49}$ Interestingly enough, a third member of the Z $\alpha$ "family" of proteins identified by sequence analysis,${ }^{50}$ the N-terminal domain of the vaccina virus E3L protein, is interferon resistant. ${ }^{49}$ 
These studies not only suggest biologically relevant functional roles for Z-DNA; they also infer possible consequences if DNA is "over" stabilized in the Z conformation. These include errors in translation due to the inappropriate substitution of guanine for adenine in gene expression, malapropos negative and positive regulation of gene promoters, and possible deleterious effects in the pathogenic response system. 


\subsection{Overview}

We are interested in the role of C8-arylated purine adducts in the role of hydrazine carcinogenesis. Unmodified and modified oligonucleotides (oligos) containing several C8arylguanine adducts have been prepared; the synthetic details will be discussed elsewhere. ${ }^{51}$ Structural and dynamic properties of these oligos were analyzed using experimental and computational methods. This work focuses on the computation approaches: molecular dynamic (MD) simulations and quantum mechanics (QM) computations, as well as the corresponding experimental techniques, circular dichroism (CD), thermal denaturation profiling and nuclear magnetic resonance (NMR) spectroscopy. Both from a computational and experimental standpoint, our findings show that the C8-guanine modified oligonucleotides tend to stabilize the Z-DNA conformer at the expense of the B-DNA conformer.

The application of computational tools to biomolecular analysis has increased in recent years due to significant advances in the quality of both software and hardware. Classical molecular dynamics is one such tool; it has been applied to many different structures and processes and has become a powerful instrument in reproducing, predicting, and complementing experimental data including thermal denaturation melting temperature $\left(T_{m}\right), N M R$, infrared

radiation (IR), kinetics, atomic resolution measurements, site-directed mutagenesis studies ${ }^{52}$, and $\mathrm{CD}^{53}$. The structure, motion, stability, and flexibility of nucleic acids are sequence specific, as are many of their roles in biological processes. No single experimental technique is able to completely describe the dynamic structure of DNA; however, MD simulations can provide an exhaustive theoretical description. Advances in force field reliability and MD simulation methodology, as well as the availability of fast workstations and increased accessibility to super 
computing facilities, have resulted in breakthroughs in computation techniques as applied to nucleic acids.

MD simulations of increasingly intricate systems including DNA-drug complexes,${ }^{54}$ DNA triplexes, ${ }^{55}$ DNA quadruplexes,${ }^{53}$ and DNA-protein complexes ${ }^{56}$ have been performed. These studies have brought about an increased understanding in nucleic acid structure and motion as a function of sequence and environment. In this work we used MD methodology as a supplement and complement to experimental data. The structural characteristics and relative stabilities of the unmodified and C8-guanine modified oligonucleotides in the B and Z-DNA conformations were also determined.

The sequence studied here is $\mathrm{d}(\mathrm{CGCGCGCGCG})_{2}(\mathbf{C G})$. This sequence was chosen mainly for its propensity towards Z-DNA formation. Polymorphism and the structure of Z-DNA itself are just two of the various sequence dependent features of DNA. Circular dichroism studies of $(\mathrm{CG})_{\mathrm{n}}$ and $(\mathrm{GC})_{\mathrm{n}}$ sequences in solution have demonstrated that a purine 5 ' start in alternating purine-pyrimidine sequences shifts the conformational equilibrium from left-handed to right-handed respectively. ${ }^{57}$ The energetics for CG and GC blocks in the right and left-handed conformations are dissimilar. The two base pairs of a CG unit have a small twist angle and a large shear resulting in the interstrand cytosines stacking with each other and the guanines forming intrastrand interactions with the sugars. The GC block, on the other hand, displays less shear and a high twist angle between adjacent base pairs, which results in less favorable stacking in the Z-DNA conformation. Conversely, the degree of stacking in right-handed DNA favors the GC dinucleotide unit over the CG dinucleotide unit. CG base pairs are highly polarized; consequently, electrostatic interactions between partial atomic charges are of great significance. These interactions lead to a preference for positive slide and negative slide conformations in CG 
and GC steps, respectively. ${ }^{58}$ Poly CG Z-DNA can accommodate this preferential combination. In fact, when two adjoining steps have very different conformational preferences, this incompatibility can actually lead to unusual structures such as Z-DNA.

As a result of this work, parameters for C8-phenylguanine, C8-p-tolylguanine, and C8-phydroxymethylphenylguanine have been developed and added to the Cornell 95 force field. ${ }^{59}$ The modified DNA's, d(CGCGCG*CGCG $)_{2}\left(\mathbf{C G}^{*}\right)$ where $\mathrm{G}^{*}$ is $\mathrm{C} 8$-phenylguanine $\left(\mathbf{C G}^{\mathbf{8}-\mathrm{Ph}}\right)$, C8-tolylguanine ( $\left.\mathbf{C G}^{\mathbf{8}-\mathrm{Tol}}\right)$, and C8-hydroxymethylphenylguanine $\left(\mathbf{C G}^{\mathbf{8}-\mathrm{Hmp}}\right)$ were constructed from the unmodified $\mathbf{C G}$ sequence by replacing the hydrogen atoms at the C8 position of G6 and G16 with a phenyl, tolyl, or hydroxymethylphenyl moiety. All sequences, both the modified and unmodified in the B or Z-DNA conformations, demonstrated structural and energetic stability over the course of a $2 \mathrm{~ns}$ production MD simulation. The phenyl and tolyl modifications of G6 and G16 served to stabilize the $\mathrm{Z}$ conformation of $\mathbf{C G}^{*}$ over the $\mathrm{B}$ form, where as the hydroxymethylphenyl adduct and CG were found to favor the B-DNA conformation. Structural analysis of the models reveals the phenyl ring lying in the major groove of $\mathbf{C G}^{*}$ in the B-DNA form, stacking under C5. This is sterically unfavorable when compared to the Z-DNA form, where the phenyl ring lies outside of the helix.

The experimental component of this work would not be possible without the C8phenylguanine phosphoramidite. To this end, a new route to C8-arylguanine derivatives was developed. ${ }^{51,60}$ Figure 1.5 outlines the synthetic scheme of C8-phenyl-2'-deoxyguanosine. The synthesis is the effort of a colleague, and will not be presented here. ${ }^{51}$ 
<smiles>CC(C)n1cnc2c(=O)[nH]c(N)nc21</smiles><smiles>Nc1nc2c(nc(Br)n2C2CC(O)C(CO)O2)c(=O)[nH]1</smiles><smiles>Nc1nc2c(nc(-c3ccccc3)n2C2CC(O)C(CO)O2)c(=O)[nH]1</smiles><smiles>[R20]CC1OC(n2c(-c3ccccc3)nc3c(=O)[nH]c(NC(=O)C(C)C)nc32)CC1O[R2]</smiles>

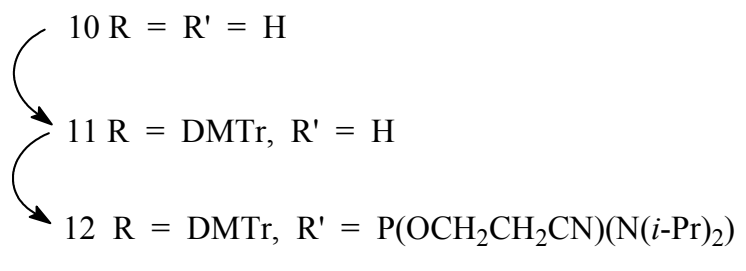

Figure 1.6. Synthetic scheme for the synthesis of the phosphoramidite of C8-phenyl-2'deoxyguanosine.

Chapter 2 describes the techniques utilized in the experimental section of this work.

Results and discussion pertaining to the experimental procedures and data are presented in Chapter 3. In Chapter 4 the theoretical background of the computational methods is presented. Results and discussion of the computational studies are set forth in Chapter 5, and Chapter 6 provides a brief conclusion. 


\section{CHAPTER 2}

\section{Experimental Techniques}

DNA assumes a variety of conformations; equilibrium between these conformations is a function of base composition, base sequence and environmental factors. In this work we examine conformational changes and relative stabilities of $\mathrm{B}$ and Z-DNA resulting from C8-aryl guanine modification. We employed several experimental techniques: thermal denaturation, circular dichroism, and nuclear magnetic resonance spectroscopy. The information gleaned from these techniques taken in total is much greater than the sum of their parts. This chapter will discuss these techniques as they apply to our work and nucleic acids in general.

\subsection{Thermal Denaturation Profiling}

Thermal denaturation measures the temperature dependence of the transition between double-stranded (ds) and single-stranded (ss) DNA. From analysis of the data one can extract thermodynamic properties. Comparison of thermal denaturation data from various sequences provides a means to measure the relationship of structure with stability.

Denaturation refers to the loss of native higher order structure as shown in Figure 2.1. The denaturation of double-stranded DNA is easily followed spectroscopically. The purine and pyrimidine bases in DNA absorb ultraviolet (UV) light maximally at a wavelength of approximately $260 \mathrm{~nm}$. In double-stranded DNA, however, the absorption is decreased due to base-stacking interactions. When DNA is denatured, these interactions are disrupted and an 
increase in absorbance is seen. This change is called the hyperchromic effect. The extent of the effect can be monitored as a function of temperature.

By increasing the temperature, the absorbed UV light increases through a series of sharp jumps. At some characteristic value, the sharpness of the jumps is reminiscent of a first order transition. This is called the melting temperature, $\mathrm{T}_{\mathrm{m}}$, and represents the point in the transition where the two phases of the complex are in equilibrium. Figure 2.2 depicts this phase transition.

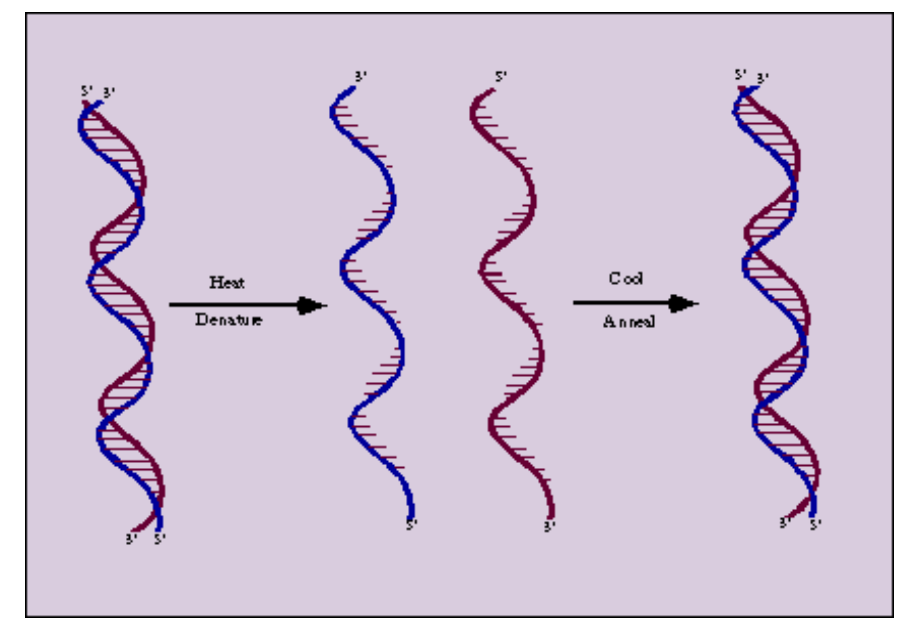

Figure 2.1. Representation of DNA thermal denaturation/renaturation.

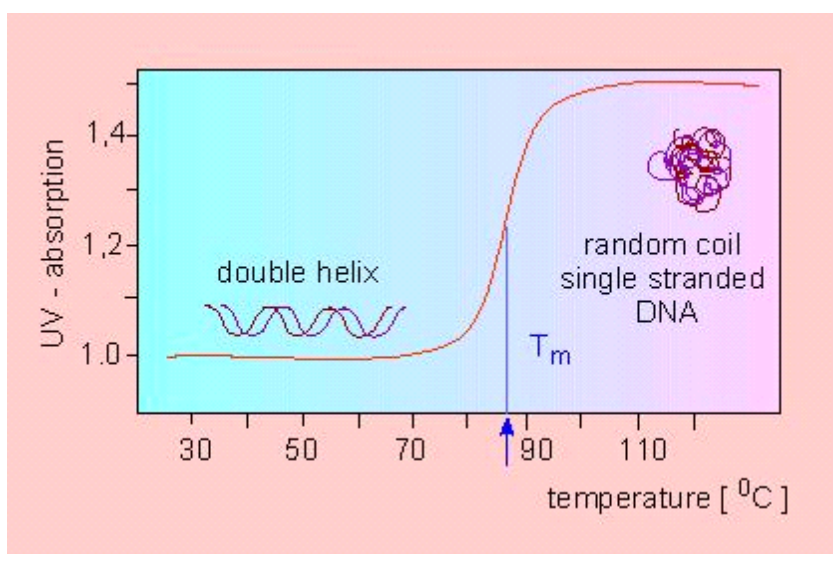

Figure 2.2. A one-step melting curve. 
Melting temperature is characteristic for any given DNA molecule under specified solution conditions. The chemical nature of the solvent, as well as the identities and concentrations of ions in the solution are all factors that influence $\mathrm{T}_{\mathrm{m}}$. Cations stabilize doublestranded DNA; therefore, $T_{m}$ increases with salt concentration. Also, divalent cations such as magnesium are more effective at stabilizing DNA duplexes than monovalent cations. ${ }^{61}$ Duplex stability is directly proportional to charge screening; therefore, the type of buffer used in sample preparation can influence melting behavior. Some have found significant deviations in melting temperature for oligonucleotides made up in ( $m$-sulfonatephenyl)phosphate trisodium (tris) versus sodium phosphate buffer, as much as $10^{\circ} \mathrm{C}$ less for those samples in the tris buffer. ${ }^{62}$ More efficient charge screening by hard ions such as sodium relative to soft ions such as tris may be responsible for this effect. ${ }^{62}$ When thermal denaturation is used to access stability relative to DNA conformation, the spectra are typically collected as a function of solution conditions that induce a known or desired conformation and the melting temperatures are compared. For example, by comparing melting temperatures of a 2-aminofluorene (2-AF) modified oligonucleotide in varying salt and ethanol concentrations it was shown that those conditions which promoted Z-DNA formation stabilized that conformation relative to the B form. ${ }^{63}$ Melting is a co-operative phenomenon; thus, melting curves increase in steepness as the length of the oligonucleotides increase. ${ }^{61}$ This results in lower melting temperatures for shorter oligos relative to longer ones of similar base content etc. Base composition also exerts a large influence on $\mathrm{T}_{\mathrm{m}}$. DNA samples that are high in AT content have relatively lower melting temperatures; DNA samples that are high in GC content have relatively higher melting temperatures. ${ }^{61}$ More energy is required to disrupt the three hydrogen bonds of a GC base pair than is required to disrupt the two hydrogen bonds of an AT base pair. Also, more energy is 
necessary to disrupt the stacking interactions involving GC base pairs than is required to disrupt stacking interactions involving AT base pairs. ${ }^{64}$ Comparison of melting curves gives a measure of relative stability between complexes. Table 2.1 below contains the melting temperatures of various G8-modified bases paired with the four natural bases and incorporated into duplex oligonucleotides.

Table 2.1. Melting temperatures in units of degrees Celsius for various G8-modified bases paired with the four natural bases and incorporated into duplex oligonucleotides.

\begin{tabular}{|c|c|c|c|c|c|c|}
\hline \multicolumn{4}{|c|}{$\begin{array}{l}\text { 5'GCA ATG GAX } \underline{X} \text { GCC TCT A3' } \\
\text { 3'CGT TAC CTY CGG AGA T5' }\end{array}$} & \multicolumn{3}{|c|}{$\begin{array}{l}\text { 5'GCG CCX } \underline{X} \text { GCG GTG3' } \\
\text { 3'CGC GGY CGC CAC5' }\end{array}$} \\
\hline $\mathbf{Y}$ & $\mathbf{X}=\mathbf{G}$ & $\mathrm{X}=\mathrm{G}^{8-\mathrm{Br}}$ & $X=8-0 \times 0 G$ & $\mathbf{X}=\mathbf{G}$ & $\mathbf{X}=\mathbf{G}^{8-\mathrm{Me}}$ & $X=G^{8-P h}$ \\
\hline $\mathrm{C}$ & $62^{\mathrm{a}}$ & $56^{\mathrm{a}}$ & $60^{\mathrm{a}}$ & $67.3^{\mathrm{b}}$ & $61.8^{\mathrm{b}}$ & $62.5^{\mathrm{d}}$ \\
\hline $\mathrm{A}$ & $55^{\mathrm{a}}$ & $49^{\mathrm{a}}$ & $55^{\mathrm{a}}$ & $60.4^{\mathrm{b}}$ & $55.6^{\mathrm{b}}$ & $59.1^{\mathrm{d}}$ \\
\hline $\mathrm{T}$ & $54^{\mathrm{a}}$ & $56^{\mathrm{a}}$ & $50^{\mathrm{a}}$ & $58.5^{\mathrm{b}}$ & $53.1^{\mathrm{b}}$ & $59.2^{\mathrm{d}}$ \\
\hline $\mathrm{G}$ & $55^{\mathrm{a}}$ & $\mathrm{n} \cdot \mathrm{c}^{\mathrm{a}, \mathrm{c}}$ & $54^{\mathrm{a}}$ & $55.4^{\mathrm{b}}$ & $57.9^{\mathrm{b}}$ & $63.7^{\mathrm{d}}$ \\
\hline
\end{tabular}

${ }^{\mathrm{a}}$ Samples were made up in $100 \mathrm{mM}$ sodium phosphate $\mathrm{pH} 7.0 .{ }^{65}{ }^{\mathrm{b}}$ Samples were prepared in $10 \mathrm{mM}$ sodium cacodylate buffer $\mathrm{pH} 7.0$ with $100 \mathrm{mM} \mathrm{NaCl}$ and $1 \mathrm{mM}$ EDTA. ${ }^{66}{ }^{\mathrm{c}}$ Not cooperative. ${ }^{65}{ }^{\mathrm{d}}$ Samples were prepared in $10 \mathrm{mM}$ sodium cacodylate buffer $\mathrm{pH} 7.0$ with $100 \mathrm{mM} \mathrm{NaCl}$ and 1 mM EDTA. ${ }^{67}$

It is also possible to obtain the thermodynamic properties of a process $(\Delta \mathrm{G}, \Delta \mathrm{H}, \Delta \mathrm{S})$ from $T_{m}$ data by performing a van't Hoff analysis. Both the enthalpy $(\Delta H)$ and entropy $(\Delta S)$ of denaturation have been extracted for various duplex oligonucleotides using thermal denaturation data. ${ }^{66,67}$ The van't Hoff equation relates the equilibrium constant to temperature and allows one to extract $\Delta \mathrm{H}$ and $\Delta \mathrm{S}$ for the transition and to calculate duplex stability at any temperature using the equations $\Delta \mathrm{G}^{\circ}=\Delta \mathrm{H}^{\circ}-\mathrm{T} \Delta \mathrm{S}^{\circ}$ and $1 / \mathrm{T}_{\mathrm{m}}=\left(\mathrm{R} / \Delta \mathrm{H}^{\circ}\right) \ln \left(\left[\mathrm{C}_{\mathrm{T}}\right] / 4\right)+\Delta \mathrm{S}^{\circ} / \Delta \mathrm{H}^{\circ}$ where $\left[\mathrm{C}_{\mathrm{T}}\right]$ is the total concentration of single-strands and the strands are non-self-complementary. ${ }^{68}$ Calculations are 
based on the slope and intercept of a plot of $1 / T_{m}$ vs $\ln \left(\left[C_{T}\right] / 4\right)$ and yield the van't Hoff transition enthalpies $\left(\Delta \mathrm{H}^{\circ}\right)$, entropies $\left(\Delta \mathrm{S}^{\circ}\right)$ and free energies $\left(\Delta \mathrm{G}^{\circ}\right){ }^{67}$ 


\subsection{Circular Dichroism Spectroscopy}

The CD spectra of A and B-DNA typically show positive ellipticity between 280-300 nm. An inverted $\mathrm{CD}$ spectrum in that range provided the first evidence for the existence of Z-DNA. ${ }^{31}$ This study was performed on poly CG sequences in a solution with high salt concentration. The subsequent crystallization of $\mathrm{d}(\mathrm{CG})_{3}{ }^{23}$ revealed the physical reason for the unusual spectrum, a left-handed double helix as opposed to the right-handedness found in B-DNA. CD data was also used to demonstrate that 5-methyl-2'-deoxycytosine $\left(\mathrm{C}^{5-\mathrm{Me}}\right)$ stabilizes Z-DNA and facilitates the transition from B to Z-DNA at nearly physiological $\mathrm{pH}^{69}$ Circular dichroism provides a means to follow the conformation of chiral molecules such as DNA; it also can afford a means to measure relative amounts of each conformer.

Circular dichroism spectroscopy is a light absorption spectroscopic technique that measures the difference in absorbance of right and left-circularly polarized light by a substance $^{70,71}$ The differential absorption of radiation polarized in two directions as a function of frequency is called dichroism. When applied to circularly polarized light, this is called circular dichroism. When the light passes through an optically active medium, it is partially absorbed. The degree of absorption is a function of the direction of the polarization of the light. Historically, ellipticity is known as the unit of circular dichroism and it is defined as the tangent of the ratio of the minor to major elliptical axis. $\mathrm{CD}$ is now measured as the difference in absorbance of right and left-circularly polarized light as a function of wavelength, but the unit ellipticity persists.

In order to observe optical activity in solution, a substance must be either chiral itself or attached to a chiral compound. Nucleic acids are composed of a phosphate sugar backbone, the 
sugars of which are chiral. Due to the helical structure of double-stranded DNA, CD

spectroscopy is extremely useful in following structural alterations such as changes in the sugarphosphate backbone geometry, base stacking interactions and handedness. For these reasons, CD spectroscopy is the perfect tool for monitoring the B-Z transition in DNA, the dramatic changes in conformation: right to left-handedness and anti to syn about the N-glycosidic bond (purines only) result in a near inversion of the spectrum in the $280-300 \mathrm{~nm}$ range. Figure 2.3 illustrates this phenomenon.

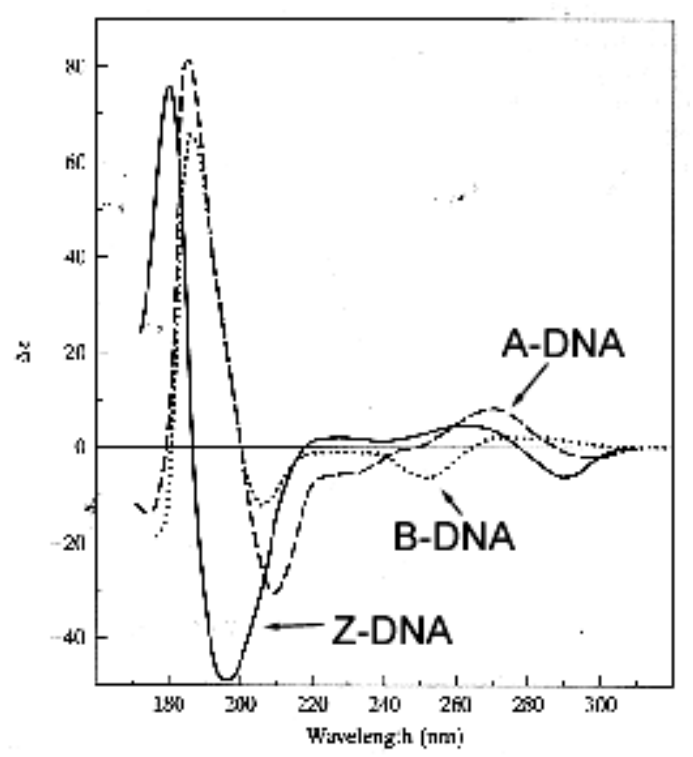

Figure 2.3. $\mathrm{CD}$ spectra of poly $\mathrm{d}(\mathrm{CG})$-poly $\mathrm{d}(\mathrm{GC})$ in the $\mathrm{A}, \mathrm{B}$ and Z-DNA conformations. Reprinted with permission from Riazance, J.H., Baase, W.A., Johnson Jr, W.C., Hall, K., Cruz, P., Tinoco Jr, I. Evidence for Z-form RNA by Vacuum UV Circular Dichroism. Nucleic Acids Res. (1985) 13:4983-4989.

As is the case in thermal denaturation studies, when CD is used to monitor changes in structural conformation of DNA, the spectra are typically collected while varying the solution conditions. For instance, to monitor the conformational changes illustrated in Figure 2.3 above, the B-DNA data was collected in low salt aqeous buffer, the Z-DNA data was collected in 2M salt and the A-DNA data was collected in ethanoic solution. CD follows changes in secondary 
structure of nucleic acids as the solution conditions vary, it is sensitive to salt and temperature in that the spectra will reflect the conditions that favor a particular conformation. CD is also used to follow the denaturation of unusual structures such as the transitions from triplex to duplex to coil.

$\mathrm{CD}$ data has been used to characterize the conformation of various sequences prone to ZDNA formation including $\mathrm{d}\left(\mathrm{C}^{5-\mathrm{Me}} \mathrm{G}^{8-\mathrm{Br}} \mathrm{C}^{5-\mathrm{Me}} \mathrm{G}^{8-\mathrm{Br}} \mathrm{CGCGCG}\right),{ }^{65} \mathrm{~d}\left(\mathrm{C}^{5-\mathrm{Me}} \mathrm{GC}^{5-\mathrm{Me}} \mathrm{GCGCGCG}\right),{ }^{65}$ $\mathrm{d}\left(\mathrm{CGCG}^{8-\mathrm{Me}} \mathrm{CG}\right),{ }^{18}$ and poly d(CG) modified with $2-\mathrm{AF} .{ }^{63}$ Poly d(AC) poly d(GT) sequences were also studied by circular dichroism. Two conformational transitions were induced by high salt or ethanol solutions; one of which displayed a negative ellipticity at $278 \mathrm{~nm}$ corresponding to the spectra characteristic of the Z-DNA conformation. ${ }^{27}$ Möller et al. monitored the influence of bromination on spectroscopic properties of poly $\mathrm{d}(\mathrm{CG}) .^{72}$ They measured the degree of transition from B to Z-DNA by monitoring the increase in negative ellipticity at $290 \mathrm{~nm}$ as well changes in intensity at $260 \mathrm{~nm}$ and found that bromination alters the active equilibrium of B and Z-DNA conformers, shifting it toward but not "freezing" it in the Z form. The disappearance of the negative band at $252 \mathrm{~nm}$, subsequent appearance of a positive band at $264 \mathrm{~nm}$ and a strong negative band at $293 \mathrm{~nm}$ characterized the $\mathrm{B}$ to $\mathrm{Z}$ transition in a study using CD to identify intermediate conformational states between the two forms. ${ }^{73}$ This study showed that the conversion from $\mathrm{B}$ to $\mathrm{Z}$ is not instantaneous and the single-strand form of DNA is not an intermediate in the process.

The application of CD spectroscopy has extended beyond that of duplex DNA to triplex ${ }^{55}$ and quadruplex ${ }^{74}$ structures, as well as protein-DNA, ${ }^{75}$ and ligand-DNA complexes. ${ }^{72}$ In addition to its sensitivity to conformation changes, CD spectroscopy is also sensitive to changes 
in sequence context and content because each base has different electronic transitions. ${ }^{76}$ For instance, the spectra of sequence isomers poly $[\mathrm{d}(\mathrm{AC}) \cdot \mathrm{d}(\mathrm{GT})]$ and poly $[\mathrm{d}(\mathrm{AG}) \cdot \mathrm{d}(\mathrm{CT})]$ are significantly different. ${ }^{77}$ Even more remarkable, the sequences AAACTAC, AAACTAT, and AAATTAC, all of which contain an AAA-TTT triplet have different characteristic shapes. ${ }^{77}$ Interpretation of CD spectra of complicated systems has historically relied heavily on correlations with structures elucidated from other techniques including high-resolution crystallography and NMR. As an increasing number of "model systems" are known for comparison, $\mathrm{CD}$ has emerged as a leading technique in the solution study of biological molecules. ${ }^{76}$ 


\subsection{Nuclear Magnetic Resonance Spectroscopy}

\subsubsection{NMR Theory}

Nuclear magnetic resonance (NMR), is a phenomenon that occurs when the nuclei of certain atoms are immersed in a static magnetic field and exposed to a second oscillating magnetic field perpendicular to the static magnetic field. In order to experience the NMR phenomenon, nuclei must have a nonzero spin. Nuclear magnetic resonance spectroscopy is the use of the NMR phenomenon to determine or study physical, chemical, and biological properties of matter. NMR spectroscopy is routinely used to study chemical structure using simple onedimensional techniques. Two-dimensional techniques are used to determine the structure of more complicated molecules, establish molecular connectivity, and conformation. These techniques have become the solution equivalent of x-ray crystallography for the determination of biomolecular structure. Time domain NMR spectroscopic techniques are used to probe molecular dynamics in solutions.

Spin is a fundamental property of nature, comes in multiples of $1 / 2$, and can be "plus" or “minus". Protons, electrons, and neutrons possess spin. Individual unpaired electrons, protons, and neutrons each possess a spin of $1 / 2$. Spin can be thought of as a magnetic moment vector, causing the proton to behave like a tiny magnet with a north (n) and south (s) pole. When the proton is placed in an external magnetic field with North $(\mathrm{N})$ and South $(\mathrm{S})$ poles, the spin vector of the particle aligns itself with the external field, just as a magnet would. There is a low energy configuration or state where the poles are aligned N-s -n-S (parallel) and a high-energy state Nn-s-S (antiparallel). When placed in a magnetic field of strength B, a particle can absorb a 
photon, of frequency, $v$. The frequency, $v$, depends on the gyromagnetic ratio, $\gamma$, of the particle as shown in Equation 2.1.

$$
v=\gamma B
$$

The proton can undergo a transition between the two energy states by the absorption of a photon. A particle in the lower energy state absorbs a photon and is promoted to the upper energy state. The energy of this photon must exactly match the energy difference between the two states or resonances. The energy, E, of a photon is related to its frequency, $\mathrm{v}$, by Plank's constant $(\mathrm{h}=$ $6.626 \times 10^{-34} \mathrm{~J} \mathrm{~s}$ ) as shown in Equation 2.2.

$$
\mathrm{E}=\mathrm{hu}
$$

The energy of the photon needed to cause a transition between two spin states is given in Equation 2.3.

$$
\mathrm{E}=\mathrm{h} \gamma \mathrm{B}
$$

When the energy of the photon matches the energy difference between the two spin states an absorption of energy occurs. The signal in NMR spectroscopy results from the difference between the energy absorbed by the spins which make a transition from the lower energy state to the higher energy state or the energy emitted by the spins which simultaneously make a transition from the higher energy state to the lower energy state. The signal is thus proportional to the population difference between the states. NMR spectroscopy is capable of detecting these very small population differences because the resonance condition is very sensitive to environment. Due to this sensitivity, small changes in structure and conformation are easily detected. 
When an atom is placed in a magnetic field, its electrons circulate about the direction of the applied magnetic field. This circulation causes a small magnetic field at the nucleus, which opposes the externally applied field. The magnetic field at the nucleus (the effective field) is, therefore, generally less than the applied field by a fraction, $\sigma$, called the shielding constant. This relationship is shown in Equation 2.4. Shielding shifts the NMR absorption upfield further than electronegativity predicts. For example, atoms above and below a benzene ring can move into a shielding region, resulting in an upfield chemical shift.

$$
\mathrm{B}=\mathrm{B}_{0}(1-\sigma)
$$

In some cases, such as for atoms in the plane of a benzene molecule, the circulation of the electrons in the aromatic $\pi$ orbitals creates a magnetic field at the hydrogen nuclei, which enhances the $B_{o}$ field. This phenomenon is called deshielding and is the result of the "ring current effect". Deshielding results in a downfield shift in NMR absorption.

The electron density around each proton in a molecule varies according to the types of nuclei and bonds in the molecule and the conformation of the molecule. The opposing field and, therefore, the effective field at each proton will vary. This is called the chemical shift phenomenon. The chemical shift of a proton is the difference between the resonance frequency of the proton and a reference proton; it is reported in ppm and given the symbol delta, $\delta$. In proton NMR spectroscopy, the standard reference is tetramethylsilane, $\mathrm{Si}\left(\mathrm{CH}_{3}\right)$, which is abbreviated TMS and arbitrarily assigned a chemical shift of 0 ppm.

Protons experiencing the same chemical and magnetic environment are called equivalent. Those protons experiencing different environments or having different chemical shifts are non- 
equivalent. If the number of interceding bonds between non-equivalent protons is less than or equal to three, there is often an observable effect on each proton's effective magnetic field by the other. This effect is called spin-spin coupling or J coupling. It can be described as the indirect coupling of protons through the intervening bonding electrons. Geminal coupling is two-bond coupling, and vicinal coupling is three-bond coupling. Figure 2.4 depicts spin coupling between two protons. Each proton has its own resonance frequency $\left(\delta_{\mathrm{A}}\right.$ and $\left.\delta_{\mathrm{X}}\right)$, and they are not equal. The spin of each proton is affected by the two orientations of the other proton through the intervening bonds. These through bond interactions result in each proton appearing as a doublet. The frequency separation between the component peaks of a multiplet is denoted by the coupling or spin-spin splitting constant, $\mathrm{J}_{\mathrm{AX}}$.

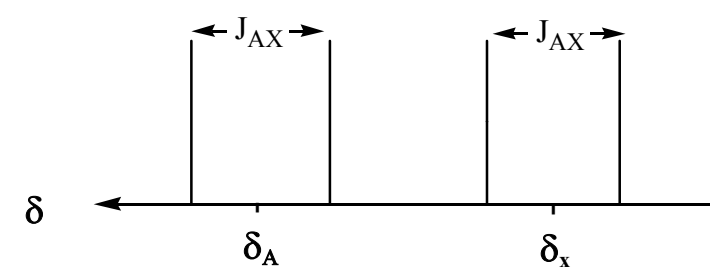

Figure 2.4. Spin coupling between two protons.

The complexity of the splitting pattern in a spectrum increases as the number of $\mathrm{X}$ nuclei increases. The multiplicity of the peak is equal to $2 \mathrm{nI}+1$, where $\mathrm{n}$ is the number of nuclei and $\mathrm{I}$ is the spin of the splitting nuclei. The following table contains a few examples. 
Table 2.2. Pascal's triangle.

\begin{tabular}{|l|l|c|}
\hline Configuration & Multiplicity & Peak Ratios \\
\hline $\mathrm{A}$ & 1 & 1 \\
\hline $\mathrm{AX}^{\mathrm{a}}$ & 2 & $1: 1$ \\
\hline $\mathrm{AX}_{2}$ & 3 & $1: 2: 1$ \\
\hline $\mathrm{AX}_{3}$ & 4 & $1: 3: 3: 1$ \\
\hline $\mathrm{AX}_{4}$ & 5 & $1: 4: 6: 4: 1$ \\
\hline $\mathrm{AX}_{5}$ & 6 & $1: 5: 10: 10: 5: 1$ \\
\hline $\mathrm{AX}_{6}$ & 7 & $1: 6: 15: 20: 15: 6: 1$ \\
\hline
\end{tabular}

${ }^{\mathrm{a}}$ Multiplicity of $\mathrm{X}$ will be 2 in all cases.

This series is called Pascal's triangle and can be calculated from the coefficients of the expansion of the binomial equation shown below:

$$
(\mathrm{a}+\mathrm{b})^{\mathrm{n}}
$$

where $n$ is the number of $X$ nuclei in Table 2.2 and first-order spectra $\left(\frac{\delta_{A X}}{J_{A X}} \geq 10\right)$ is assumed.

Vicinal coupling is dependent upon the dihedral angle, $\boldsymbol{\varphi}$, between two protons. Figure 2.5 illustrates the dependence of the ${ }^{1} \mathrm{H}-{ }^{1} \mathrm{H}$ coupling constant through three bonds (H-C-C-H), its magnitude is fitted by the Karplus equation stated in simplified form as ${ }^{3} \mathrm{~J}_{\mathrm{HH}}=15.3 \cos ^{2} \varphi-$ $6.2 \cos \varphi+1.5(\mathrm{~Hz}) .{ }^{61}$ For systems where the bond cannot be rotated, the coupling is useful in the determination of stereochemistry or the derivation of structural constraints. 


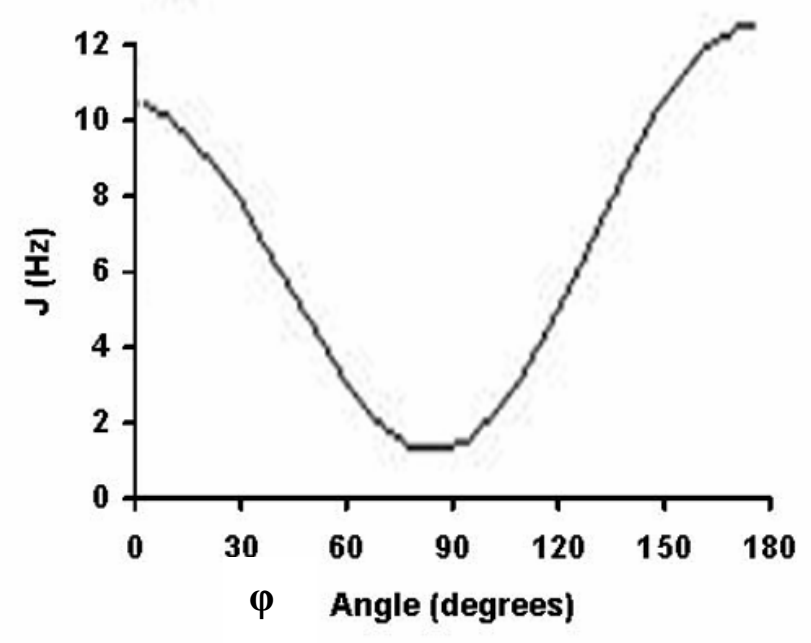

Figure 2.5. Karplus Curve demonstrating the dependence of $J$ on the dihedral angle, $\varphi$.

The other major source of structural information comes from through space dipole-dipole coupling between two protons called the nuclear Overhauser effect (nOe). The intensity of a nOe is proportional to the inverse of the sixth power of the distance separating the two protons and is usually observed if two protons are separated by $<5$ Angstroms. Thus, the nOe is a sensitive probe of short intramolecular distances. nOe's are categorized according to the location of the two protons involved in the interaction. In the case of DNA, nOe's can be between protons on the same base, adjacent bases on the same strand, or between bases on opposite strands. A network of these short inter-proton distances is useful for local structure determination and, in combination with computational methods, global structure elucidation of nucleic acids. 


\subsubsection{Biological Applications of NMR}

NMR has been applied to biological molecules since the $1950 \mathrm{~s}^{78}$ In NMR, the one dimensional proton $\left({ }^{1} \mathrm{H}\right)$ spectrum has one resonance for each chemically and magnetically distinct species of proton. Due to the size of the molecules and large number of chemically and magnetically similar protons, the one-dimensional NMR spectra of biopolymers lack the resolution or dispersion necessary to determine the individual resonance assignments necessary for structural elucidation. Because the combination of J coupling, multiplicity and nOe's can be used to establish conformation, the advent of multi-dimensional NMR has led to sequential resonance assignment procedures for both proteins and DNA's. These systematic resonance assignments rely on information obtained from J correlated spectroscopy (COSY) and nOe correlated spectroscopy (NOESY) experiments. In two dimensions the diagonal of the spectrum corresponds to a conventional one-dimensional experiment while the cross-peaks occurring off the diagonal contain information about the correlations between the resonances on the diagonal. $^{78}$ These correlations can be through-bond (COSY), or through-space (NOESY), or based on exchange [rotating frame Overhauser spectroscopy (ROESY)]. nOe's are a reliable probe of short intramolecular distances. COSY adds important information regarding bond angles and torsions and is used to confirm many of the resonance assignments.

Two continuous networks of nOe connectivities have been characterized in right-handed DNA with all anti glycosidic torsion angles. A "nOe walk" procedure evolving from crystal structure measurements and NOESY spectra has made it possible to sequentially assign resonances by "walking" up the hydrogen-bonded protons in the center of the helix and along the 
sugar-base backbone. ${ }^{79}$ Each base $\mathrm{H} 8$ or $\mathrm{H} 6$ proton is within nOe distance of both its own H1', $\mathrm{H} 2^{\prime}$ and $\mathrm{H} 2 "$ protons and those on the $5^{\prime}$ neighboring base. Connectivities between protons in a given sugar, as well as first-stage assignments of the cytosine $\mathrm{H} 5$ and $\mathrm{H} 6$ protons ${ }^{80}$ can be obtained from COSY spectra. Identification of 5' or 3' nucleotides for reference from independent criteria is possible and can serve as a starting location for the resonance assignments.

Resonance assignments for Z-DNA is somewhat more complicated due to a lack of short sequential ${ }^{1} \mathrm{H}-{ }^{1} \mathrm{H}$ distances relative to right-handed DNA. However, data computed from the crystal structure of $\mathrm{d}(\mathrm{CGCGCG})_{2}$ is sufficient for nOe resonance assignments in sequences containing alternating pyrimidine (anti) - purine (syn) dinucleotide repeats. Generally speaking, sequential connectivities are observed $5^{\prime}$ syn-anti $3^{\prime}$ but not 5 ' anti-syn $3^{\prime}{ }^{81}$ Strong intranucleotide $\mathrm{H} 8-\mathrm{H} 1$ ' nOe cross peaks serve to identify guanine in the syn conformation. Table 2.3 highlights the differences in resonance assignments for base and sugar protons in the B and Z-DNA conformations for pyrimidines (PY) and purines (PU). The dominant factor which affects the resonance assignments is the large difference in the sugar-base torsion angle $(\chi)$ of the guanine residues in the syn conformation for Z-DNA. ${ }^{82}$ Chemical shifts of a given base are affected by the complementary base, the nearest neighbor bases on the same strand, and the nearest neighbor bases on the complementary strand. ${ }^{82}$ Thus, in the case of $\mathrm{d}(\mathrm{CGCGCGCGCG})_{2}$, G6 is affected by C15 (complementary base), C5, C7, (nearest neighbor bases on the same strand) G14 and G16 (nearest neighbor bases on the complementary strand) albeit to different degrees. In the case of Z-DNA where the helical repeat is a dinucleotide unit, the effects from these "neighbors" are different from that of B-DNA where the repeating unit is a mononucleotide unit. 
Table 2.3. Typical resonance assignments for base/sugar protons, highlighting the differences between $\mathrm{B}$ and Z-DNA in bold print. ${ }^{66}$

\begin{tabular}{|l|c|l|l|l|l|l|l|l|l|}
\hline & H5 & H8/6 & H1' & H2' & H2" & H3' & H4' & H5' & H5" \\
\hline \hline B (PY) & $5.4-5.6$ & $\mathbf{7 . 9 5}$ & $5.7-6.0$ & $\mathbf{1 . 9 - 2 . 0}$ & $\mathbf{2 . 3 - 2 . 5}$ & $4.6-5.0$ & $\mathbf{4 . 2}$ & $4.1-4.2$ & $4.1-4.2$ \\
\hline B (PU) & - & 7.85 & $\mathbf{5 . 7 - 6 . 0}$ & $\mathbf{2 . 6 - 2 . 7}$ & $\mathbf{2 . 7 - 2 . 9}$ & $4.8-5.0$ & 4.2 & $4.1-4.2$ & $3.9-4.1$ \\
\hline Z (PY) & $5.2-5.4$ & $\mathbf{7 . 5 - 7 . 6}$ & $5.7-6$ & $\mathbf{1 . 6 - 1 . 8}$ & $\mathbf{2 . 5 - 2 . 8}$ & $4.8-5.0$ & $\mathbf{3 . 7 - 3 . 9}$ & $\mathbf{2 . 6 - 2 . 8}$ & $3.7-4.0$ \\
\hline Z (PU) & - & $7.9-8.0$ & $\mathbf{6 . 2 - 6 . 5}$ & $\mathbf{2 . 7 - 3 . 0}$ & $\mathbf{2 . 8 - 2 . 9}$ & $5.0-5.1$ & $4.2-4.3$ & 4.1 & $4.0-4.2$ \\
\hline
\end{tabular}

The imino and amino resonances of both $\mathrm{B}$ and Z-DNA must be acquired in $90 \% \mathrm{H}_{2} \mathrm{O}$ due to their fast rate of chemical exchange in $\mathrm{D}_{2} \mathrm{O}$. For DNA structures containing WatsonCrick base pairs, imino resonance assignments are made sequentially between adjacent base pairs and amino resonances are assigned via cross peaks from the iminos. Generally, cytosine aminos are the easiest to identify due to their relatively slower exchange time. ${ }^{83}$ On the other hand, guanine aminos exchange more rapidly, complicating resonance assignments.

It is notoriously difficult to study Z-DNA by solution NMR. ${ }^{83}$ High salt conditions generally used to promote Z-DNA formation in solution are not conducive to obtaining quality NMR specta due to aggregation, precipitation and reduced solubility. These factors cause linebroadening, ${ }^{83}$ resulting in spectra lacking the necessary clarity for unequivocal resonance assignments. Base modifications known to promote Z-DNA formation without high salt concentrations are one solution to this problem and have helped advance the study of DNA conformation by NMR. 
NMR data has been used to characterize the conformation of various sequences prone to Z-DNA formation including d(CGCG $\left.{ }^{8-\mathrm{Me}} \mathrm{CG}\right),{ }^{18}$ and d(CCATC[AF]GCTACC).

$\mathrm{d}$ (GGTAGCGATGG). ${ }^{84} \mathrm{NMR}$ was also used in the study of the $\mathrm{B}$ to $\mathrm{Z}$ transition of $\mathrm{d}\left(\mathrm{C}^{5-\mathrm{Me}} \mathrm{GC}^{5-}\right.$ ${ }^{\mathrm{Me}} \mathrm{GC}^{5-\mathrm{Me}} \mathrm{G}$ ) in varying concentrations of methanol. ${ }^{83}$ This study used methanol to avoid the problems associated with high salt concentrations in the NMR analysis of DNA's in the Z conformation. The authors found strong intranucleotide nOe crosspeaks from base protons $\mathrm{H} 8$ and $\mathrm{H} 6$ to $\mathrm{H} 2$ ' and $\mathrm{H} 2$ ' sugar protons and weak intranucleotide crosspeaks to H1' for the DNA in the $\mathrm{B}$ form. In the $\mathrm{Z}$ form, induced by a methanol concentration of $40 \%$, strong nOe intranucleotide crosspeaks were observed from base H8 to its own H1' sugar, indicative of guanine in the syn conformation. ${ }^{83}$ Also, there is only one correlation between each aromatic base proton and a sugar $\mathrm{H1}$ ' resonance, in contrast to B-DNA which has correlations from the aromatic base protons to two H1' sugar protons, its own and that of its 5' neighbor except in the case of the $5^{\prime}$ terminal base.

Unlike CD spectra, which provide overall secondary structure content, NMR provides secondary structure information that is localized to specific segments of a particular molecule. For instance, sugar pucker has been estimated from the magnitudes of the coupling constants linking $\mathrm{H} 1^{\prime}-\mathrm{H} 2^{\prime}$ and $\mathrm{H}^{\prime}-\mathrm{H} 4^{\prime}$ and application of the Karplus equation. ${ }^{61}$ In fact, the characteristic differences between certain vicinal ${ }^{1} \mathrm{H}-{ }^{1} \mathrm{H}$ coupling constants serve to differentiate the $\mathrm{C} 3$ '-endo sugar pucker predominately found in A-DNA from the $\mathrm{C} 2$ '-endo conformation customarily found in B-DNA as shown in Table 2.4. 
Table 2.4. Approximate coupling constants ${ }^{61}$ and corresponding dihedral angles ${ }^{85}$ for C 3 '-endo and $\mathrm{C} 2$ '-endo sugar puckers.

\begin{tabular}{|c|c|l|l|l|}
\hline $\begin{array}{l}\text { Coupling } \\
\text { Constant }\end{array}$ & C3'-endo (Hz) & $\begin{array}{l}\text { Dihedral Angle } \\
\text { (Degrees) }\end{array}$ & C2'-endo (Hz) & $\begin{array}{l}\text { Dihedral Angle } \\
\text { (Degrees) }\end{array}$ \\
\hline \hline${ }^{3} \mathrm{~J}_{1^{\prime} 2^{\prime}}$ & 2 & 89 & 10 & 158 \\
\hline${ }^{3} \mathrm{~J}_{1^{\prime} 2^{\prime \prime}}$ & 8 & -32 & 6 & 39 \\
\hline${ }^{3} \mathrm{~J}_{2^{\prime} 3^{\prime}}$ & 7 & 43 & 6 & -39 \\
\hline${ }^{3} \mathrm{~J}_{2^{\prime \prime} 3^{\prime}}$ & 9 & 166 & 1 & 80 \\
\hline${ }^{3} \mathrm{~J}_{3^{\prime} 4^{\prime}}$ & 8 & -158 & 1 & 96 \\
\hline
\end{tabular}

The conformation of the glycosydic bond, $\chi$, can be analyzed from the H6/H8 - H1' connectivities, and some of the phosphodiester backbone torsion angles can be determined from other appropriate three-bond connectivities. ${ }^{61}$ Coupled with molecular dynamics, the nOe and dihedral bond angle information can yield more precise details with regard to sequence specific variation in tilt, propeller twist, helical twist, groove widths and ultimately precise molecular structure. ${ }^{81}$ In correlation with the level of structural detail possible from NMR analysis, considerably more material (milligrams) and effort (requires sequence specific resonance assignments) are necessary to obtain secondary structure information than that required for other spectroscopic techniques (e.g. CD, thermal denaturation). 


\section{CHAPTER 3}

\section{Experimental Details and Results}

\subsection{Experimental Details}

Solvents and reagents were obtained from Aldrich (Milwakee, WI) and were used without purification unless otherwise noted. Methylene chloride was dried by distillation from phosphorus pentoxide. Triethylamine (TEA), pyridine, and tetrahydrofuran (THF) were dried by distillation from lithium aluminum hydride (LAH). Dimethylformamide (DMF) was purified by distillation from barium oxide. Unmodified phosphoramidite DNA bases and controlled pore glass (CPG) resins were obtained from Glen Research (Sterling, VA). Mass spectra were recorded on an Agilent 5973N (low resolution) or a Finnigan MAT 90 (high resolution) mass spectrometer.

All DNA samples were made up in phosphate buffer $\left(10 \mathrm{mM} \mathrm{NaH}_{2} \mathrm{PO}_{4}, \mathrm{pH} 7.4\right)$ and varying concentrations of sodium chloride, heated to $90^{\circ} \mathrm{C}$ for $30 \mathrm{~min}$., and then cooled slowly to room temperature. UV melting temperature experiments were conducted on the $\mathbf{C G}$ and $\mathbf{C G}^{\mathbf{8}-\mathbf{P h}}$ duplexes by monitoring absorbance at $260 \mathrm{~nm}$ using a Cary 300 spectrometer under the following conditions: $10 \mathrm{mM}$ sodium phosphate buffer, $\mathrm{pH} 7.4$, and varying $\mathrm{NaCl}$ concentrations (Table 3.1). Spectra were recorded over the temperature range of $25-90^{\circ} \mathrm{C}$ at a heating rate of $0.25^{\circ} \mathrm{C} / \mathrm{min}$.

The CD spectra were recorded on an AVIV Model 62A CD spectrometer. Solution concentrations of each oligonucleotide were approximately $25 \mu \mathrm{M}$. Unless otherwise stated, solutions were prepared in $10 \mathrm{mM}$ phosphate buffer, $\mathrm{pH} 7.4$, with varying amounts of $\mathrm{NaCl}$ 
(Figure 3.1) and annealed as described for the thermal denaturation samples. The final sample volume was $400 \mu \mathrm{L}$.

The NMR samples were prepared in $10 \mathrm{mM}$ phosphate buffer, $\mathrm{pH}$ 7.4, with $25 \mathrm{mM}, 100$ $\mathrm{mM}, 200 \mathrm{mM}$, and $1 \mathrm{M} \mathrm{NaCl}$ in $\mathrm{D}_{2} \mathrm{O}(1 \mathrm{mM}$ in duplex $)$. All NMR spectra were measured on an Inova $500 \mathrm{MHz}$ spectrometer (Department of Chemistry and Biochemistry, Florida State University, Tallahassee, FL) at $28^{\circ} \mathrm{C}$. The two-dimensional NOESY spectra of nonexchangeable protons were collected at $28^{\circ} \mathrm{C}$ with a mixing time of $150 \mathrm{~ms}$. The data were collected with $512 t_{1}$ increments and $2048 t_{2}$ complex points, each the sum of 64 transients. Data were apodized with shifted sine bell functions in both dimensions and zero filled to give a $4 \mathrm{k}$ by $1 \mathrm{k}$ data set. The two-dimension NOESY spectra of exchangeable protons were acquired on samples at $28^{\circ} \mathrm{C}$ in $10 \% \mathrm{D}_{2} \mathrm{O} / 90 \% \mathrm{H}_{2} \mathrm{O}$ solution using the $1-1$ pulse sequence, with a mixing time of $150 \mathrm{~ms}$ and a sum of 64 transients 


\subsection{Results}

\subsubsection{Themal Denaturation Profiling}

Thermal denaturation curves were obtained on solutions of $\mathbf{C G}$ and $\mathbf{C G}^{\mathbf{8 - P h}}$ at low, intermediate, and high salt concentrations. Under low salt conditions (25 mM), where the B form is expected to predominate for both oligonucleotides, the unmodified 10-mer CG displayed a well defined melting curve. Similar $\mathrm{T}_{\mathrm{m}}$ curves were also observed for $\mathbf{C G}$ and $\mathbf{C G}^{\mathbf{8 - P h}}$ at high salt levels $(4.5 \mathrm{M})$. The thermal denaturation temperatures are shown in Table 3.1. The unmodified oligonucleotide $\mathbf{C G}$ and modified oligonucleotide $\mathbf{C G}^{\mathbf{8}-\mathbf{P h}}$ gave similar curves and melting temperatures at 25 and $100 \mathrm{mM}$ sodium chloride. However, as shown by the circular dichroism and NMR data, the $\mathbf{C G}^{\mathbf{8 - P h}}$ oligonucleotide exists as a mixture of conformers at these salt concentrations, and thus, interpretation of the thermal denaturation data is not straightforward since the observed $\mathrm{T}_{\mathrm{m}}$ does not necessarily correspond to one species.

Table 3.1. Melting temperatures for $\mathbf{C G}$ and $\mathbf{C G}^{\mathbf{8 - P h}}$.

\begin{tabular}{|c|c|c|}
\hline $\mathrm{NaCl}(\mathrm{mM})$ & $\mathrm{T}_{\mathrm{m}}\left({ }^{\mathrm{o}} \mathrm{C}\right) \mathbf{C G}^{\mathbf{a}}$ & $\mathrm{T}_{\mathrm{m}}\left({ }^{\circ} \mathrm{C}\right) \mathbf{C G}^{8-\mathrm{Ph}}$ \\
\hline 25 & 64 & $64^{\mathrm{b}}$ \\
\hline 100 & 65 & $65^{\mathrm{b}}$ \\
\hline 4500 & 66 & 66 \\
\hline
\end{tabular}

${ }^{\mathrm{a}}$ All samples contained approximately $0.5 \mu \mathrm{M}$ oligonucleotide dissolved in $10 \mathrm{mM}$ phosphate buffer, $\mathrm{pH}$ 7.4. Data were collected from $20-95^{\circ} \mathrm{C}$ at a heating rate of $0.25^{\circ} / \mathrm{min}$. ${ }^{\mathrm{b}}$ At the indicated salt concentration, $\mathbf{C G}^{\mathbf{8}-\mathbf{P h}}$ was not present as a single form (see text). 


\subsubsection{Circular Dichroism}

Circular Dichroism spectroscopy can distinguish between B and Z-DNA. The CD signal of B-DNA shows positive ellipticity at approximately $280 \mathrm{~nm}$ and $220 \mathrm{~nm}$ and a negative ellipticity at approximately $248 \mathrm{~nm}$. In contrast, Z-DNA shows a negative ellipticity at approximately $295 \mathrm{~nm}$ and $248 \mathrm{~nm} \cdot{ }^{18,69,76}$ Figure 3.1a illustrates the CD spectra of CG as a function of salt concentration. The salt levels were varied from 0-4600 mM. The CG oligonucleotide, at salt concentrations below $2 \mathrm{M}$, is predominantly in the B form as indicated by the positive ellipticity at $280 \mathrm{~nm}$. Above $2 \mathrm{M}$ salt, the $\mathrm{Z}$ form predominates. Overall, these observations for the $\mathbf{C G}$ are consistent with what has been reported for related oligonucleotides. $^{18}$

The CD spectra of the modified oligonucleotide, $\mathbf{C G}^{8-\mathrm{Ph}}$, is shown in Figure 3.1b. The dependence of these spectra on salt concentration, which was varied from $0-3600 \mathrm{mM}$, is remarkably different with respect to CG. First, under low salt conditions, the $248 \mathrm{~nm}$ minimum is attenuated relative to the unmodified oligonucleotide. Second, by inspection of the spectrum between $280-295 \mathrm{~nm}$, it can be seen that roughly equivalent amounts of the B and Z-DNA forms are present at salt concentrations between 200-400 mM sodium chloride, 5-10 times less than required for $\mathbf{C G}$.

The $\mathrm{CD}$ data for $\mathbf{C G}^{\text {8-Ph }}$ is similar to other related oligonucleotides that have alternating purine/pyrimidine sequences and a C8-modified guanine. Many of these studies were conducted with poly $(\mathrm{dG}-\mathrm{dC})$ that had been treated with agents known to form $\mathrm{C} 8$-adducts such as bromine, ${ }^{69} \mathrm{~N}$-methyl-4-aminoazobenzene, ${ }^{86}$ or 2-AF. ${ }^{63}$ These systems usually require salt concentrations in the range of 1-2 M sodium chloride (closer to $2 \mathrm{M}$ for the unmodified DNA's) 
to cause a prevalence of the Z-DNA conformation. For example, at $11 \%$ incorporation of $2-\mathrm{AF}$ in poly $\mathrm{d}(\mathrm{GC})$, salt levels above $1.2 \mathrm{M}$ are required for the Z-DNA form to predominate.

a)

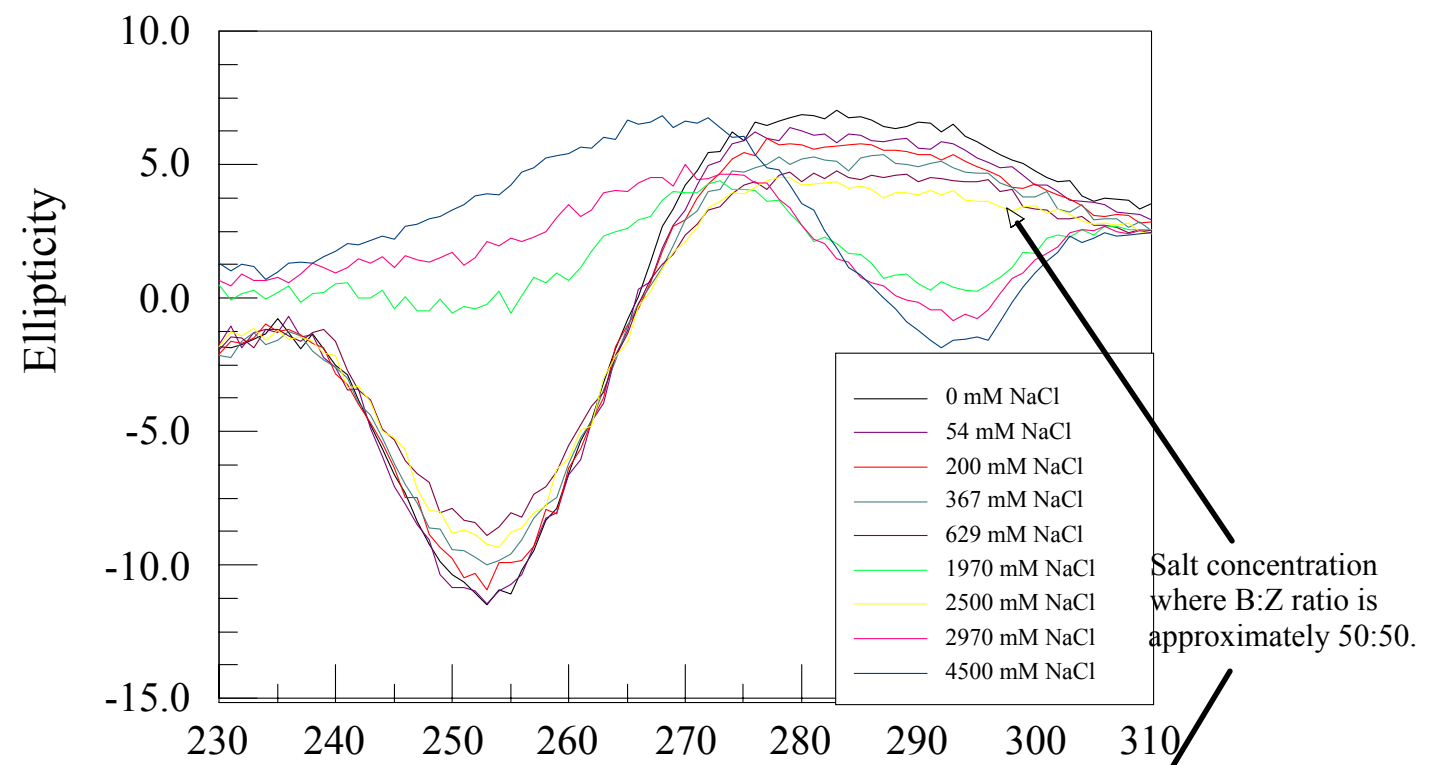

b)

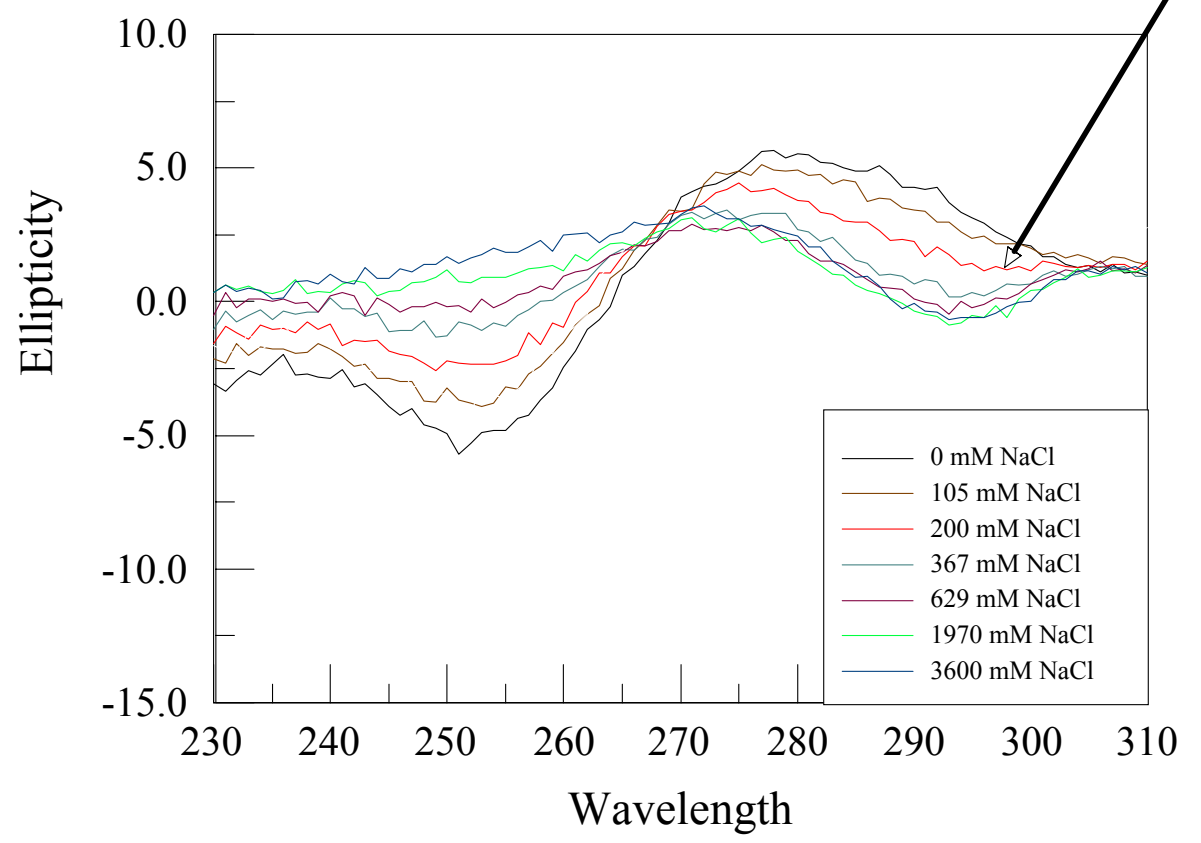

Figure 3.1. CD spectra of a) CG and b) CG8-Ph as a function of salt concentration. All spectra were recorded at $20 \mathrm{oC}$ in solutions containing approximately $25 \mathrm{mM}$ oligonucleotide, $10 \mathrm{mM}$ phosphate buffer, $\mathrm{pH}$ 7.4. 


\subsubsection{NMR}

Characteristic through-space and through-bond interactions observed as cross peaks in NOESY or COSY spectra, respectively, were used to help distinguish conformations of the duplexes. COSY and NOESY data were obtained on samples made up in buffer with low salt concentration ( $25 \mathrm{mM} \mathrm{NaCl})$ and were assigned by standard procedures. We first acquired NMR spectra of the unmodified oligonucleotide (CG) to serve as a reference spectrum for the B-DNA form. Chemical shift assignments are shown in Table 3.2. Two-dimensional NOESY spectra of exchangeable protons displayed minimal dispersion of resonances belonging to imino and amino proton because of the repeated base sequence. ${ }^{87}$ As a result, only two distinct imino proton resonances (at 12.99 and $13.02 \mathrm{ppm}$ ) were observable. Likewise, only two resonances attributable to base paired amino protons $(8.35$ and $8.37 \mathrm{ppm})$ and non-base paired amino protons (6.42 and $6.46 \mathrm{ppm})$ were detected. Sequential assignments were made chiefly from NOESY spectra in $\mathrm{D}_{2} \mathrm{O}$. The pattern of intra- and inter-residue nOes was consistent with the oligonucleotide adopting a B-DNA structure. Similar measurements were then made under high salt conditions, which favor Z-DNA formation. However, we were unable to obtain clear NMR spectra of $\mathbf{C G}$ in the Z-DNA form because the high salt concentrations required to convert $\mathbf{C G}$ completely into the Z-DNA form $(4.5 \mathrm{M} \mathrm{NaCl})$ produced significant line broadening. 
Table 3.2. Chemical Shifts of $\mathbf{C G}$ in the B-DNA conformation given in units of ppm. ${ }^{\text {a }}$

\begin{tabular}{|c|c|c|c|c|c|c|c|c|c|}
\hline $\begin{array}{c}\text { Base } \\
\text { Number }\end{array}$ & H5 & H8/6 & H1' & H2' & H2' & H3' & H4' & H5' & H5" \\
\hline \hline C1 & 5.88 & 7.61 & 5.78 & 1.95 & 2.41 & 4.88 & 4.20 & 4.16 & 4.16 \\
\hline G2 & - & 8.01 & 5.94 & 2.65 & 2.8 & 5.02 & 4.40 & 4.14 & 4.06 \\
\hline C3 & 5.45 & 7.35 & 5.77 & 1.94 & 2.41 & 4.88 & 4.16 & 4.13 & 4.13 \\
\hline G4 & - & 7.93 & 5.91 & 2.66 & 2.75 & 5.02 & 4.39 & 4.14 & 4.06 \\
\hline C5 & 5.41 & 7.33 & 5.72 & 2.00 & 2.42 & 4.88 & 4.23 & 4.16 & 4.16 \\
\hline G6 & - & 7.92 & 5.90 & 2.66 & 2.74 & 5.02 & 4.40 & 4.14 & 4.06 \\
\hline C7 & 5.41 & 7.34 & 5.73 & 1.92 & 2.35 & 4.85 & 4.20 & 4.13 & 4.13 \\
\hline G8 & - & 7.93 & 5.92 & 2.70 & 2.76 & 5.02 & 4.40 & 4.14 & 4.06 \\
\hline C9 & 5.48 & 7.36 & 5.85 & 1.94 & 2.36 & 4.84 & 4.20 & 4.16 & 4.16 \\
\hline
\end{tabular}

${ }^{a}$ Sample conditions were $1 \mathrm{mM} \mathrm{CG}, 10 \mathrm{mM}$ phosphate buffer, $\mathrm{pH}=7.4,100 \mathrm{mM}$ sodium chloride, and $28^{\circ} \mathrm{C}$.

NMR spectra of the 8-phenyl modified oligonucleotide $\mathbf{C G}^{\mathbf{8}-\mathbf{P h}}$ were acquired at $25 \mathrm{mM}$, $100 \mathrm{mM}, 200 \mathrm{mM}$, and $1 \mathrm{M}$ sodium chloride. The chemical shift assignments, determined at $1 \mathrm{M} \mathrm{NaCl}$, are shown in Table 3.3. In particular, strong intranucleotide G2-H1'/G2-H8, G4H1'/G4-H8, G8-H1'/G8-H8, and G10-H1'/G10-H8 cross-peaks were observed, consistent with a Z-DNA conformation. ${ }^{88}$ Deoxyguanosine residues adopted the syn conformation, identified by 
strong intranucleotide $\mathrm{H} 8-\mathrm{H1}$ ' nOe cross peaks, and the cytosines favored the anti conformation about the glycosidic bond.

Table 3.3. Chemical Shifts of $\mathbf{C G}^{\text {8-Ph }}$ in the Z-DNA conformation given in units of ppm . ${ }^{\text {a }}$

\begin{tabular}{|c|c|c|c|c|c|c|c|c|c|}
\hline $\begin{array}{l}\text { Base } \\
\text { Number }\end{array}$ & H5/Ph & H8/6 & H1' & H2' & H2" & H3' & H4' & H5' & H5" \\
\hline $\mathrm{C} 1$ & 5.90 & 7.58 & 5.86 & 1.77 & 2.55 & 4.73 & 3.78 & 2.72 & 3.19 \\
\hline $\mathrm{G} 2$ & - & 7.93 & 6.35 & 2.87 & 2.87 & 5.12 & 4.28 & 4.17 & 4.05 \\
\hline $\mathrm{C} 3$ & 5.24 & 7.49 & 5.85 & 1.82 & 2.74 & 4.93 & 3.89 & 2.72 & 3.90 \\
\hline G4 & - & 7.91 & 6.33 & 2.82 & 2.82 & 4.95 & 4.24 & 4.13 & 4.01 \\
\hline $\mathrm{C} 5$ & 5.34 & 7.56 & 5.91 & 1.87 & 2.76 & 4.94 & 3.90 & 2.71 & 3.90 \\
\hline $\mathrm{G} 6^{8 \mathrm{Ph}}$ & $\begin{array}{l}7.82, \\
7.67,7.51\end{array}$ & - & 6.4 & 2.81 & 2.81 & 4.96 & 4.41 & 4.23 & 4.02 \\
\hline $\mathrm{C} 7$ & 5.24 & 7.57 & 5.99 & 1.86 & 2.78 & 5.10 & 3.90 & 2.72 & 3.74 \\
\hline G8 & - & 7.93 & 6.36 & 2.88 & 2.88 & 5.13 & 4.29 & 4.18 & 4.18 \\
\hline C9 & 5.36 & 7.55 & 5.87 & 1.82 & 2.79 & 4.94 & 3.88 & 2.72 & 3.88 \\
\hline G10 & & 8.03 & 6.39 & 3.33 & 2.57 & 4.96 & 4.33 & 4.18 & 4.15 \\
\hline
\end{tabular}

${ }^{\text {a }}$ Sample conditions were $1 \mathrm{mM} \mathbf{C G}^{\mathbf{8}-\mathbf{P h}}, 10 \mathrm{mM}$ phosphate buffer, $\mathrm{pH}=7.4,1 \mathrm{M}$ sodium chloride, and $28^{\circ} \mathrm{C}$.

The assignments of the sugar- $\mathrm{H} 2^{\prime} / \mathrm{H} 2 "$ were made using the nOe correlations from the base protons to these sugar protons. All C-H2' protons in $\mathbf{C G}^{\mathbf{8}-\mathrm{Ph}}$ were upfield of the corresponding resonance of the unmodified oligonucleotide (B-DNA), and the $\mathrm{C}-\mathrm{H} 2 "$ and $\mathrm{G}-\mathrm{H} 2$ '/H2" protons 
were all downfield. In the case of the $\mathrm{C}-\mathrm{H} 2 "$ protons, exclusive of the terminal bases, the observed downfield shift ( $Z$ versus B) was substantial (0.3-0.4 ppm range). Correlations between the G-H8/H1' and C-H4'/H5'/H5" protons were observed (illustrated in Figure 3.2), as expected for a Z-DNA and used to assign the latter resonances. The shifts determined for C$\mathrm{H} 4^{\prime} / \mathrm{H} 5^{\prime} / \mathrm{H} 5^{\prime \prime}$ in $\mathbf{C G}^{8-\mathrm{Ph}}$ were all upfield of the corresponding protons in the unmodified oligonucleotide (C-H4' protons roughly 0.4 ppm upfield shifted, C-H5' approximately $0.2 \mathrm{ppm}$ upfield, and C-H5" greater than $1.4 \mathrm{ppm}$ ). Assignments of the G-H4'/H5'/H5" protons were based on NOESY correlations between G-H2' and G-H4', G-H3' and G-H5'/H5", and COSY correlations from G-H2" -> G-H3' -> G-H4' -G-H5'/H5". Thus, unlike CG, it was possible in the case of $\mathbf{C G}^{8-\mathrm{Ph}}$ to assign the $\mathrm{G}-\mathrm{H} 3^{\prime} / \mathrm{H} 4^{\prime} / \mathrm{H} 5^{\prime} / \mathrm{H} 5^{\prime \prime}$ and $\mathrm{C}-\mathrm{H} 3^{\prime} / \mathrm{H} 4^{\prime} / \mathrm{H} 5^{\prime} / \mathrm{H} 5^{\prime \prime}$ protons unequivocally because of their upfield shifts as shown in Figure 3.2. Finally, as in the case of CG, the imino and amino protons were not resolved; only two distinct imino protons were observable (12.34 and $13.18 \mathrm{ppm})$. Likewise, only two distinct base paired amino protons (8.47 and $8.32 \mathrm{ppm}$ ) and non-base paired amino protons (6.80 and 6.65) were observable. 

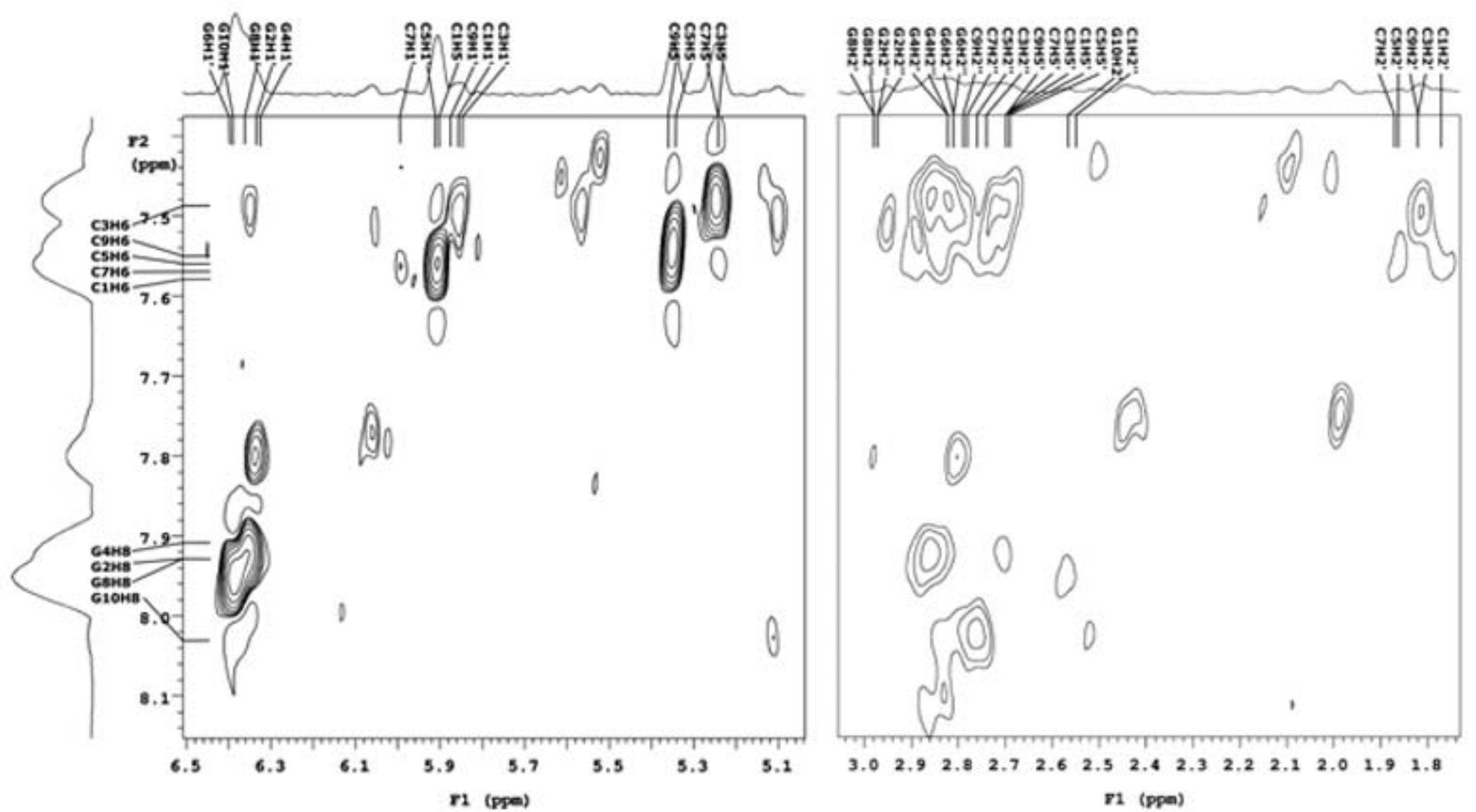

Figure 3.2. Aromatic and sugar regions of the NOESY spectrum of $\mathbf{C G}^{\mathbf{8 - P h}}$ showing the correlations between $\mathrm{GH} 8$ - H1' and CH6 $\mathrm{CH} 5$ (left) and the sugar protons (right). The sample was made up in $1 \mathrm{M} \mathrm{NaCl}, 10 \mathrm{mM}$ sodium phosphate buffer, $\mathrm{pH} 7.4$ at $20^{\circ} \mathrm{C}$. 
The spectra recorded at lower salt concentrations $(25,100$, or $200 \mathrm{mM} \mathrm{NaCl})$ were quite complex, making full assignment problematic. We tentatively attributed the complexity to the presence of all three forms of $\mathbf{C G}^{\mathbf{8 - P h}}$ (ss, B, and Z) in the sample and attempted to identify resonances for phenyl protons associated with each form. Assignment of the phenyl protons in the $\mathrm{Z}$ form of $\mathbf{C G}^{\mathbf{8}-\mathbf{P h}}$ was made on the basis of their observed chemical shifts in $1 \mathrm{M} \mathrm{NaCl}$. At the lower salt concentrations, in addition to those previously recorded shifts, additional resonances attributable to phenyl protons were observed (Figure 3.3). The additional sets of phenyl resonances have been assigned to the B and single-stranded forms (Table 3.4). The numbering scheme for $\mathbf{C G}^{\mathbf{8 - P h}}$ is shown in Figure 3.4 .

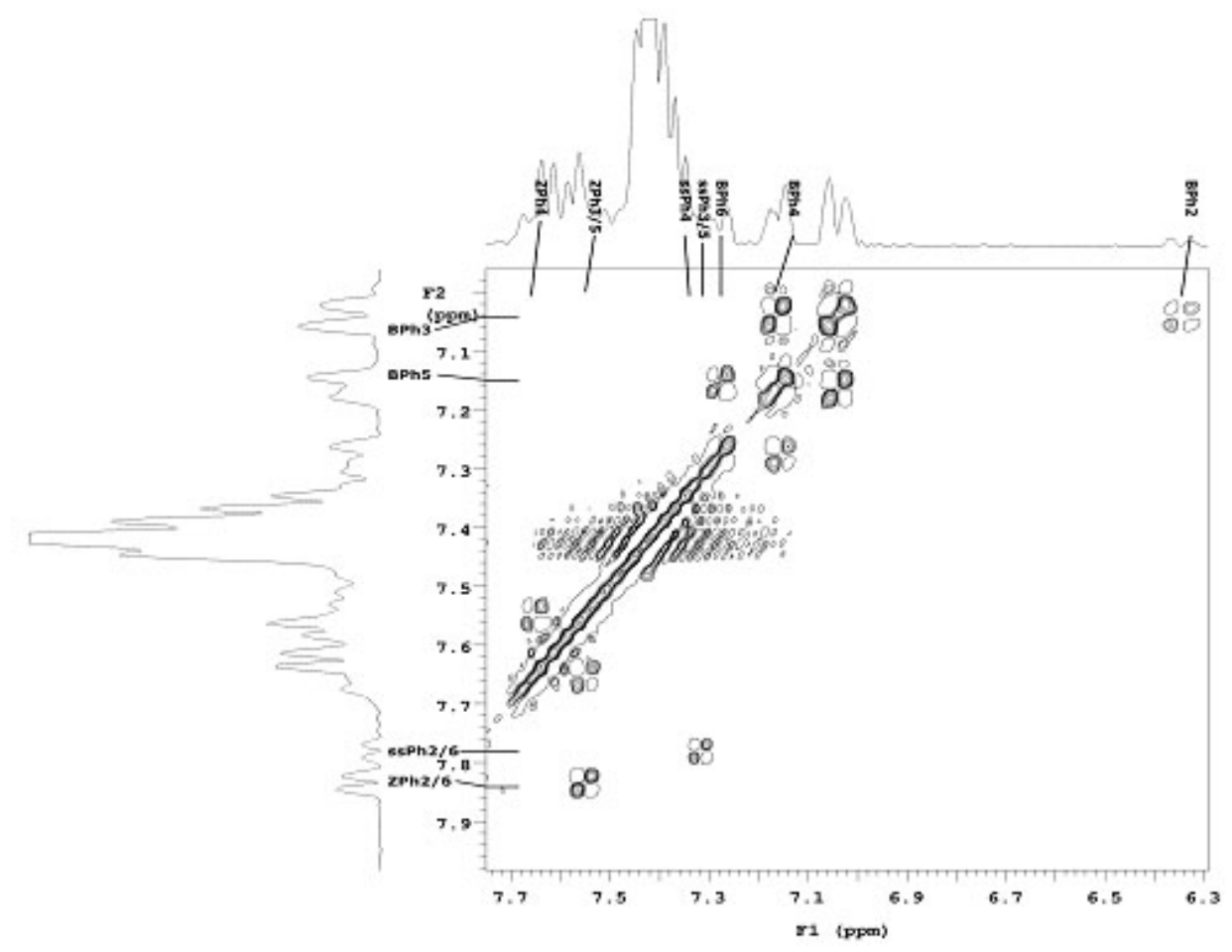

Figure 3.3. Aromatic region of the COSY spectrum of $\mathbf{C G}^{\mathbf{8 - P h}}$ showing the phenyl protons in the single-stranded (ss), B-DNA (B) and Z-DNA (Z) forms. The sample was made up in 100 $\mathrm{mM} \mathrm{NaCl}, 10 \mathrm{mM}$ sodium phosphate buffer, $\mathrm{pH} 7.4$ at $20^{\circ} \mathrm{C}$. 
Table 3.4. Single-stranded, B, and Z-DNA chemical shifts of the C8-phenyl protons of $\mathbf{C G}^{\mathbf{8}-\mathrm{Ph}}$ given in units of ppm. ${ }^{a}$

\begin{tabular}{|l|l|l|l|}
\hline & H-10/14 (ortho) & H-11/13 (meta) & H-12 (para) \\
\hline Single-Stranded & 7.78 & 7.32 & 7.33 \\
\hline B-DNA & $6.35 / 7.28$ & $7.04 / 7.15$ & 7.17 \\
\hline Z-DNA & 7.84 & 7.55 & 7.65 \\
\hline
\end{tabular}

${ }^{\mathrm{a}}$ Sample conditions were $1 \mathrm{mM} \mathbf{C G}^{\text {8-Ph }}, 10 \mathrm{mM}$ phosphate buffer, $\mathrm{pH}=7.4,100 \mathrm{mM}$ sodium chloride, and $28^{\circ} \mathrm{C}$.

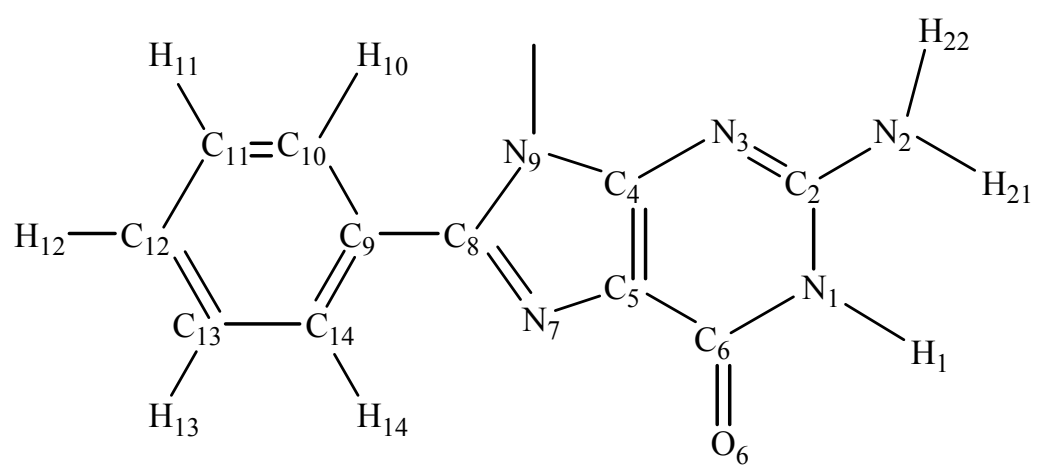

Figure 3.4. Numbering scheme for $\mathbf{C G}^{\mathbf{8 - P h}}$.

The chemical shifts of the phenyl group in the single-stranded form were similar to the ZDNA form, although they are shifted upfield a small amount. The chemical shifts for the phenyl group in the B form display a chemical shift range of nearly $1 \mathrm{ppm}$. As indicated in Table 3.4, the chemical shift of the phenyl-H10 proton is far upfield at $6.35 \mathrm{ppm}$ (Figure 3.3). The upfield shift of the phenyl-H10 is substantial, appearing approximately $1.5 \mathrm{ppm}$ upfield of the same proton of the $\mathrm{Z}$ form and is likely due to the location of this proton over $\mathrm{C} 5$ as shown in Figure 5.14. The phenyl-H11 is also shielded by C5, although not to the same extent. Likewise, the 
phenyl-H12, H13, and H14 are less shielded by $\mathrm{C} 5$ than the phenyl-H10 proton but are still upfield of the corresponding resonances in either the single-stranded or Z forms of $\mathbf{C G}^{\mathbf{8}-\mathbf{P h}}$.

By acquiring two-dimensional spectra at varying temperatures, one can frequently resolve ambiguities due to spectral overlap. ${ }^{81}$ To this end, a variable temperature experiment was conducted, and the phenyl resonances ascribed to each of the forms were monitored over the temperature range of $10-50{ }^{\circ} \mathrm{C}$. At the lower temperature, the resonances assigned to the $\mathrm{Z}$ form predominated. As the temperature increased, so did the resonances correlating with the B form; the resonances attributed to the single-stranded form also increased, but not at the rate of the B. At $50^{\circ} \mathrm{C}$, the $\mathrm{B}$ form predominated. This behavior is in agreement with the assignments based on similar studies conducted with C8-methylguanine modified oligonucleotides. ${ }^{18}$ It was not possible to accurately quantitate the relative amounts of the $\mathrm{B}, \mathrm{Z}$, and single-stranded forms due to overlap of the latter with other resonances. However, the increase in the B form is unequivocal as most of the phenyl resonances associated with the B form do not overlap other resonances. 


\subsection{Discussion}

Only one example of a C8-phenylguanine modified oligonucleotide has been studied. ${ }^{16}$ In that case, the C8-phenylguanine modification repotedly decreased the thermal denaturation temperature. In the present case, we do not see any difference in the thermal denaturation temperature between $\mathbf{C G}$ and $\mathbf{C G}^{\mathbf{8 - P h}}$. Under low salt conditions, $\mathbf{C G}^{\mathbf{8 - P h}}$ is not in one form; rather it is a mixture of three conformations, though, as the melting temperature is approached, the B form predominates. Consequently, the reported melting temperature may mainly reflect the melting of the B-DNA. Nevertheless, significant ambiguities exist and whether the C8phenylguanine modification destabilizes oligonucleotides in the B-DNA form will require additional studies. Under high salt conditions, where the Z-DNA form of both $\mathbf{C G}$ and $\mathbf{C G}^{\text {8-Ph }}$ is present, the melting temperatures measured were identical and suggest that the Z-DNA conformations of $\mathbf{C G}$ and $\mathbf{C G}^{\text {8-Ph }}$ are of similar stability. This is reaffirmed in the free energy calculations discussed in Chapter 5. This result is not unexpected, as in the Z-DNA conformation; the phenyl group of the modified oligonucleotide is pointed away from the duplex and cannot sterically destabilize the duplex.

Though the thermal denaturation data cannot be used to quantitate the effect of the phenyl modification on the B-DNA form, the C8-phenylguanine modification does stabilize the Z-DNA form of $\mathbf{C G}^{\text {8-Ph }}$ relative to the Z-DNA form of $\mathbf{C G}$. This is clearly demonstrated by the CD data that show that a 1:1 mixture of the B and Z-DNA forms in CG requires a salt concentration 5-10 times higher than required for $\mathbf{C G}^{\mathbf{8}-\mathrm{Ph}}$. This result is comparable to what has been observed in recent studies with alternating purine/pyrimidine oligonucleotides with $\mathrm{C} 8$-bromo ${ }^{65}$ or C8methyl ${ }^{18}$ substitution although these latter two modifications appear to be even more effective at shifting the $\mathrm{B} / \mathrm{Z}$ equilibrium in favor of the Z-DNA form. The difference between the bromo or 
methyl substituents and the phenyl substituent may be due to the spherical nature of the former and the planer nature of the latter. In addition, since the bromo and methyl substituents have less surface area than a phenyl group, unfavorable hydrophobic interactions may be less for the bromo and methyl substituents than for the phenyl group. Nevertheless, it is interesting that the Z-DNA form is adopted under physiological conditions, as the steric affects of the C8-guanine substitution by bromine, methyl or phenyl must overcome the unfavorable electrostatic interactions present in the $\mathrm{Z}$ form. Bromo, methyl, and phenyl modifications, therefore, may be useful in studying the factors that affect the $\mathrm{B} / \mathrm{Z}$ equilibrium.

The information obtained from the NMR experiments further support the CD data regarding the $\mathrm{B} / \mathrm{Z}$ equilibrium. The NMR spectra acquired at $1 \mathrm{M}$ sodium chloride confirms the complete conversion to the $\mathrm{Z}$ form. Furthermore, at lower salt concentrations (25-200 mM) the CD data suggest and the NMR experiments unequivocally show that $\mathbf{C G}^{\mathbf{8 - P h}}$ exists as a mixture of B and Z-DNA. The NMR results also demonstrate the presence of a third species, tentatively assigned as the single-strand form of $\mathbf{C G}^{\text {8-Ph }}$. At least two additional possibilities exist for this third form. First, Z-DNA is known to exist in different substates $\left(\mathrm{Z}_{\mathrm{I}} / \mathrm{Z}_{\mathrm{II}}^{89}\right.$ and $\left.\mathrm{Z}[\mathrm{WC}]^{90}\right)$. It is not clear that the phenyl groups in these different forms would have different chemical shifts but if so, then two different Z-DNA forms could be present. However, the assignment of the three forms to single-stranded, B-DNA, and Z-DNA seems the most likely possibility based on the variable temperature NMR experiment (data not shown). Previous studies have shown that the Z-DNA form is preferred at low temperature. ${ }^{83}$ Upon raising the temperature, the concentration of the B-DNA conformation increases at the expense of the Z. The single-stranded form also increases in concentration with temperature, albeit more slowly than the B-DNA form until the 
thermal denaturation temperature is reached. The variable temperature NMR data for $\mathbf{C G}^{\mathbf{8}-\mathbf{P h}}$ are in agreement with this.

Due to the complexity of the NMR spectra at low salt concentrations, a complete molecular model cannot, at this time, be derived from nOe based distance constraints. However, the limited structural information we have for $\mathbf{C G}^{\mathbf{8 - P h}}$, derived from the NMR data, is consistent with the structural models shown and discussed in Chapter 5. In particular, the chemical shifts of the phenyl protons ascribed to the $\mathrm{B}$ and $\mathrm{Z}$ forms are consistent with the molecular models, and the latter help to provide an explanation for the observed chemical shifts. In addition, the data suggest that the B form adopts an anti conformation about the modified base's glycosidic bond. This conformation places the phenyl group in the major groove, and all five phenyl protons are in different environments leading to the prediction that all five will have different chemical shifts. Finally, the alternative $s y n$ conformation of the phenyl-modified guanines in the B-DNA conformer cannot be rigorously excluded. However, since the loss of the $\mathrm{C}: \mathrm{G}^{8-\mathrm{Ph}}$ base pair would significantly destabilize the duplex, this seems unlikely. 


\section{CHAPTER 4}

\section{Theoretical Background}

\subsection{Historical Overview}

This chapter describes the quantum mechanics (QM), molecular mechanics (MM), molecular dynamics (MD), and free energy calculations used in the current work to model modified and unmodified poly d(CGCGCGCGCG) $)_{2}$ oligonucleotides. The QM calculations were performed using Gaussian 98, and the MM, MD, and free energy calculations were performed with the Amber Versions $6^{91}$ or $7^{92}$ suite of molecular simulation programs.

In the early 1960's, pioneering work on the development of force fields began independently in the laboratories of Shneior Lifson, Harold Scheragam, and Norman Allinger. ${ }^{93}$ In 1973 Norman Allinger described the modeling of hydrocarbons with a new force field, MM1. ${ }^{94} \mathrm{He}$ is considered an innovator in the field of computational chemistry and the development of molecular mechanics force fields. The first molecular dynamics study of a protein $^{95}$ was published in 1977, this was one of many contributions involving Martin Karplus. Peter Kollman was a major force in extending the application of force field methodology and computer simulation to biomolecular systems; a preliminary description of the Amber force field for protein/DNA calculations was published in $1981 .^{96}$ Around the same time other force fields including CHARMM ${ }^{97}$ were developed. The late 1970's saw the advent of commercial vendors such as Tripos, in fact the number of companies specializing in computational chemistry has increased from 3 in 1979 to well over 20 today. ${ }^{98}$

More powerful computing resources, improved force fields, more accurate methods, and collaboration between research groups are all factors that have contributed to advancements in 
the simulation of nucleic acids in solution. So called "second generation" force fields such as Cornell et al. ${ }^{59}$ and CHARMM ${ }^{99}$ have benefited from higher-level QM calculations and more extensive testing. Modern simulations have been able to follow the conversion of A to B-DNA in the duplex form, ${ }^{100}$ the $\mathrm{A}$ to $\mathrm{B}$ transition of triplex DNA. ${ }^{101} \mathrm{MD}$ also been used to demonstrate the environmental dependence of nucleic acid structure by simulating the stabilization of A-DNA in ethanoic solution. ${ }^{102}$ Free energy calculations have also become commonplace and are used in a wide variety of applications including protein-ligand binding, ${ }^{103}$ DNA-ligand interactions,${ }^{54}$ stability studies ${ }^{104}$ and RNA-protein interactions. ${ }^{105}$ In fact, just as NMR can be considered the solution equivalent of $x$-ray crystallography, computer simulations have become a viable virtual counterpart. 


\subsection{General Force Field Parameter Development and Quantum Mechanics Calculations}

In order to conduct simulations with C8-modified guanines, suitable parameters for the modified bases had to be developed and incorporated into the Cornell 95 force field. ${ }^{59}$ The force field contains the necessary building blocks for the calculations of energy and force. The general principle in developing force field parameters for new fragments or molecules is to use analogy as much as possible. ${ }^{92}$ Parameter development starts with a two dimensional sketch of the relevant fragment; the assignment of atom types follows. If no appropriate atom type exists in the force field, a new atom type must be created. Having chosen or created one or more atom types, the bond, angle, and dihedral parameters need to be created. Bond angles and lengths are often obtained from the literature, by analogy to similar existing force field parameters, or from QM calculations. The relative conformational energies of a molecule are determined primarily from the dihedral parameters, in conjunction with the partial atomic charges and Van der Waals parameters. $^{92}$ QM calculations are used to generate dihedral potential energy profiles and subsequent determination of the lowest energy conformer and torsional barrier. QM output is also used to calculate partial atomic charges. To assess the degree of reasonableness of the values resulting from these calculations, $\mathrm{x}$-ray data, if available, are an excellent comparison. One can also look for discrepancies/similarities between the QM calculations for the given atoms and similar atoms in the force field. Similarities suggesting the new parameters developed from the QM calculations are at least consistent with the force field.

In this work Gaussian $98^{106}$ was used for the QM calculations required for force field parameter development. Gaussian is a connected series of programs for performing semiempirical, density functional theory, and ab initio molecular orbital calculations. The principles of quantum mechanics are used as a basis for the computations performed by the program. In 
quantum chemistry, a system is described by a wavefunction, which can be found by solving the Schrödinger equation:

$$
\hat{H} \Psi=E \Psi
$$

where $\mathrm{H}$ is the Hamiltonian for the system described by the following expression:

$$
\hat{H}=\sum_{i=1}^{N}\left(-\frac{\hbar^{2}}{2 m_{i}} \nabla_{i}^{2}\right)+\frac{1}{2} \sum_{i=1}^{N} \sum_{j \neq i}^{N} \frac{Z_{i} Z_{j}}{4 \pi \epsilon_{0}\left|\mathbf{r}_{i}-\mathbf{r}_{j}\right|}
$$

$N$ is the number of particles in the system, $m$ the mass of a particle $i, \Psi$ is an N-body

wavefunction, $\mathbf{r}$ denotes spatial positions, $\hbar$ is Dirac's constant (Planck's constant divided by $2 \pi$ or approximately $\left.1.05 \times 10^{-34} \mathrm{Js}\right), \nabla^{2}$ is the Laplacian, $\varepsilon_{0}$ is the permittivity of free space (a physical constant equal to approximately $8.85 \times 10^{-12} \mathrm{Farad} / \mathrm{m}$ ), and $Z$ denotes the charges of the individual particles $i$ and $j$. $E$ denotes the energy of either the ground or an excited state of the system. This equation relates the stationary states of the system and their energies to the Hamiltonian operator, which can be viewed as the recipe for obtaining the energy associated with a wavefunction describing the positions of the nuclei and electrons in the system. This Hamiltonian neglects at least two effects. First, although the speed of an electron in a hydrogen atom is less than $1 \%$ of the speed of light, relativistic mass corrections can become appreciable for the inner electrons of heavier atoms. Secondly, the spin-orbital effects are neglected. In practice, the Schrödinger equation often cannot be solved exactly and approximations have to be made. The ab initio approach makes no use of empirical information, except for the fundamental constants of nature such as the mass of the electron, Planck's constant etc., that are required to arrive at numerical predictions. In spite of the necessary approximations, ab initio theory has the conceptual advantage of generality, and the practical advantage that (with experience) its successes and failures are more or less predictable. 
In order to perform a calculation in Gaussian, a theoretical model or model chemistry must be selected. A theoretical model is comprised of a method (e.g. Hartree-Fock or MP2 etc.) and a basis set. In this work the Hartree Flock (HF) Self Consistent Field (SCF) method was chosen. Hartree Fock theory is one of the simplest approaches for solving the Schrödinger equation and assumes a single-determinant form for the wavefunction. An important property of the SCF method is that its solutions satisfy the Variation Principle, which states that the expectation value of the energy evaluated with an inexact wavefunction is always higher than the exact energy. Consequently, the lowest energy is associated with the best approximate wavefunction, and energy minimization is equivalent with wavefunction optimization.

The 6-31G* basis set represents each inner-shell orbital with one contracted Gaussian function that is a linear combination of six primitive Gaussian functions and each valence orbital with two basis functions, one contracted Gaussian function that is a linear combination of three primitive Gaussians and one primitive Gaussian function. In addition, six d-type Gaussian functions for each nonhydrogen atom in the second or third period are included in the basis set. Basis sets such as $6-31 \mathrm{G}^{*}$ are said to be polarized basis sets. $\mathrm{HF} / 6-31 \mathrm{G}^{*}$ is considered a good enough method for the determination of the geometries of organic molecules.

In this work, Gaussian was used to perform single point energy calculations and geometry optimizations. The energy calculations were used for input into the RESP (restrained electrostatic potential) module of Amber. RESP fits the quantum mechanically calculated electrostatic potential (esp) at molecular surfaces using an atom-centered point charge model. The geometry optimizations were used to determine the torsional barrier for rotation about the C8-arylguanine bond. The Gaussian calculations were performed using a closed shell model, sometimes referred to as a restricted calculation. In this type of calculation, each electron pair is 
forced into a single spatial orbital as opposed to unrestricted calculations which use separate spatial orbitals for the spin up and spin down electrons. 


\subsection{Molecular Mechanic Calculations}

Despite the expected accuracy of QM treatments, the complexity of the calculations generally limits their use in biological molecular modeling applications to the development or evaluation of empirical potentials or the study of relatively small molecules. Since QM calculations are generally not possible for biological systems, simplifications must be made in order to approach their study from a computational standpoint. A well developed approach is molecular mechanics (MM).

Molecular mechanical force fields are based on the theory that the potential surfaces and physical properties of molecules may be calculated using classical mechanics functions. A potential energy function is a mathematical equation that allows for the potential energy, $U$, of a chemical system to be calculated as a function of the physical interactions that dictate its threedimensional structure. A molecule is thought of as a collection of atoms, which interact with each other in a way that can be described by simple potential energy functions or force fields. Amber is based upon a valence force field, and the total potential energy potential, $U_{t o t}$, which describes intramolecular (based on the covalent structure of the molecule) and intermolecular interactions between all the atoms and molecules of a system, is given in Equation 4.3.

$$
\begin{aligned}
U_{\text {total }}=\sum_{\text {bonds }} k_{r}\left(r-r_{0}\right)^{2}+\sum_{\text {angles }} k_{\theta}\left(\theta-\theta_{0}\right)^{2}+\sum_{\text {dihedrals }} \sum_{n} \frac{V_{n}}{2}[1+\cos (n \varphi-\gamma)] \\
+\frac{1}{V D W_{\text {scale }}} \sum_{j=1}^{\text {bondsbonds }} \sum_{i>j}^{*} \varepsilon^{*}\left[\left(\frac{R_{i j}^{*}}{r_{i j}}\right)^{12}-\left(\frac{R_{i j}^{*}}{r_{i j}}\right)^{6}\right]+\frac{1}{E E L_{\text {scale }}} \sum_{j=1}^{\text {atomsatoms }} \sum_{i>j} \frac{q_{i} q_{j}}{r_{i j} \varepsilon}
\end{aligned}
$$

The first three terms, which describe bond, angle, and dihedral potentials may be thought of as covalent interactions. The bond-stretching and angle-bending terms are treated harmonically, which keeps the bond lengths, $r$, and angles, $\theta$, near their equilibrium values, $r_{0}$ 
and $\theta_{0} . k_{r}$ and $k_{\theta}$ are bond stretching and angle bending constants associated with the bond and angle terms. The dihedral term includes the force constant, $V_{n}$ that dictates the magnitude of torsional barrier, the periodicity, $n$ that indicates number of cycles per $360^{\circ}$, and a phase, $\gamma$ that dictates the location of the maxima in the dihedral energy surface. Improper torsional angles, in which the atoms are not serially bonded to each other, but branched as shown in Figure 4.1, use the same potential function as torsional angles, they are typically used to preserve planarity of specific ring fragments. The convention is that the central atom is listed in the third position of the dihedral, $\mathbf{K}$ in the figure below.

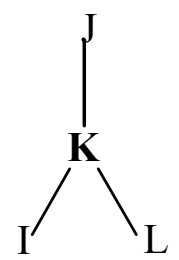

Figure 4.1. Improper torsion angle I-J-K-L.

The next two terms, the Van der Waals interactions represented by a Lennard-Jones 6-12 potential and electrostatic potentials modeled by Coulombic interactions of atom-centered point charges, describe the non-bonded interactions. These terms are considered the most critical energy terms because of the strong influence of the environment on the properties of macromolecules, as well as the large number of nonbond interactions occurring in molecules themselves. ${ }^{107}$ Parameters associated with these terms are Van der Waals well depth for given atom, $\varepsilon^{*}=\left(\varepsilon_{\mathrm{ii}}{ }^{*} \varepsilon_{\mathrm{jj}}{ }^{*}\right)^{1 / 2}$, the minimum interaction radius, $R_{i j} *=\left(R_{i i}{ }^{*} R_{j j}{ }^{*}\right)^{1 / 2}$, dielectric constant, $\varepsilon$, and the partial atomic charges, $q_{j}$. Electrostatic and Van der Waals interactions are calculated between atoms in different molecules or for atoms in the same molecule separated by at least three bonds. Those non-bonded interactions separated by exactly three bonds, "1-4 interactions", 
are reduced by the application of a scale factor. A 6-12 function is used to represent the Van der Waals interactions: the attractive London dispersion force between two atoms is described by a $\mathrm{r}^{-6}$ term and the repulsive interaction is given by $\mathrm{a}^{-12}$ term. A 6-12 function is considered too repulsive for shorter nonbonded interactions, ${ }^{108}$ but the more realistic 6-exponential form is prohibitive from a computational time standpoint, hence the scale factor. Likewise, the 1-4 electrostatic interactions are also scaled to compensate for neglecting the charge redistribution during a pairwise interaction. ${ }^{108}$

Molecular mechanics is a useful tool in molecular modeling; for example, minimization removes gross steric overlaps and relaxes strain. Molecular mechanics is also necessary prior to molecular dynamics simulations; however, the limits in sampling, likelihood of minimizing to a local minimum versus the global minimum, and assumptions regarding temperature $(0 \mathrm{~K})$ limit its usefulness as applied to biological molecules. Starting from many initial geometries is an option in overcoming some of these obstacles, but it is not a practical solution. MM produces a motionless molecule of idealized geometry. In reality, the molecules undergo thermal motions and constantly change their geometry; therefore, MD provides a more realistic representation. 


\subsection{Molecular Dynamics Simulations}

Molecular Dynamics (MD) is a computational method that calculates the time dependent behavior of a molecular system by numerically integrating Newton's equations of motion for all of the atoms of a system. ${ }^{107} \mathrm{MD}$ simulations have provided detailed information on the fluctuations and conformational changes of biological molecules such as proteins and nucleic acids. To start a simulation, velocities, $v_{i}$, are assigned to all $\mathrm{N}$ particles with mass, $m_{i}$, from the Maxwell distribution at given temperature, T (Equation 4.4).

$$
\frac{1}{2} \sum_{i}^{N} m_{i} v_{i}^{2}=\frac{3 N k T}{2}
$$

In principle, the classical equations of motion are deterministic, that is, once the initial coordinates and velocities are known, the coordinates and velocities at a later time can be calculated by first determining the force on each particle, $F_{i}$, as a function of time, and acceleration, $a_{i}$, as shown in Equation 4.5.

$$
\boldsymbol{a}_{i}=\frac{\boldsymbol{F}_{i}}{m_{i}}=-\frac{1}{m_{i}} \frac{\partial U}{\partial r_{i}}
$$

The Hamiltonian of the system as a function of both coordinates, $r$, and momenta, $p$, in terms of kinetic and potential energy is given in Equation 4.6, where $U(r)$ has the form of the Amber energy function (Equation 4.3).

$$
H(\boldsymbol{r}, \boldsymbol{p})=\frac{1}{2} \sum_{i=1}^{N} \frac{\boldsymbol{p}_{i}^{2}}{m_{i}}+U(\boldsymbol{r})
$$

The Velocity Verlet Integrator ${ }^{109,110}$ is one of a variety of first order integration algorithms that stores positions, velocities and acceleration all at the same time and is typically used to integrate the equations of motion in order to obtain the coordinates for the next step as follows. 


$$
\boldsymbol{q}_{i}(t+\delta t)=\boldsymbol{q}_{i}(t)+\delta t \boldsymbol{v}_{i}(t)+\frac{\delta t^{2}}{2 m_{i}} \boldsymbol{F}_{i}(t)
$$

where $q$ is the position, $v$ the velocity, $F$ the force, $m$ is masss, and $t$ denotes time. This technique takes a two step approach to updating the velocities. In the first step, the velocities are advanced from time $t$ to time $\left(t+\frac{\delta t}{2}\right)$. Then forces, $F$, are updated for the new atomic positions, and the velocities are updated from time $\left(t+\frac{\delta t}{2}\right)$ to time $(t+\delta t)$ and are calculated by Equation 4.8.

$$
\boldsymbol{v}_{i}(t+\delta t)=\boldsymbol{v}_{i}(t)+\frac{\delta t}{2 m_{i}}\left[\boldsymbol{F}_{i}(t+\delta t)+\boldsymbol{F}_{i}(t)\right]
$$

The resulting set of positions and velocities, $R=\left\{q\left(t_{1}\right), q\left(t_{2}\right) \ldots v\left(t_{1}\right), v\left(t_{2}\right)\right\}$, are referred to as the dynamics trajectory. Thus, with this method both the positions and velocities are known at the same time step, which has several advantages over other algorithms. The main disadvantage is that it is computationally more expensive relative to simpler procedures. ${ }^{111}$

DNA's are highly charged molecules and their representation with simple potential functions presents special difficulties. In essence, nucleic acids are constantly hydrated, so an explicit representation of solvent is necessary. Water plays a structural role by acting as either an H-bond donor or acceptor. It also has polarization effects by screening the electrostatic interactions due to its high dielectric constant ( $~ 80)$. In addition to solvent, the high charge density from the phosphate backbone and strong salt dependence of nucleic acids structure require explicit representation of counterions. Even with explicit representation of water and ions, the complexity of the calculation centers on the proper evaluation of the pairwise nonbonded and Coulombic interactions. Hence, in the case of empirical derived force fields, the 
importance of choosing one that has been properly parameterized to model the structures of interest is key.

Simulations with explicit solvent have been performed on DNA molecules for almost 20 years, the first occurring in $1985 .{ }^{112}$ They were limited to a short time scale $(\sim 100 \mathrm{ps})$ and typically displayed anomalous structure, ${ }^{113}$ such as base fraying, which demonstrated the necessity for inclusion of more accurate representation of solvent. More recent simulations suggest the importance of properly treating the long-ranged electrostatic interactions, ${ }^{114}$ even with the inclusion of explicit solvent and counter-ions. Standard approximations to limit the effective range of the high charge and large electrostatic interactions found in nucleic acids are used for more affordable computing but lead to instability during molecular dynamics. ${ }^{115}$ Detailed treatment of solvent, as well as long range electrostatics has become a reality thanks to increased computer power and better approximations.

Even for explicit systems, it is desirable to use the simplest and most efficient model possible. The most commonly used explicit water models are rigid three point models such as TIP3P. ${ }^{116}$ These hypothetical models are the simplest that can be applied while still retaining most of the structural, energetic, and dynamic properties of water. The drawbacks to these simple models including an underestimation of compressibility and lack of tetrahedral water structure. More complicated models give better fit but with a significant increase in computational cost.

Early simulations of nucleic acids in explicit solvent used a vacuum interface to surround a "block" of solvated nucleic acid. By the end of a 100 ps simulation under these conditions, the terminal residues on one side of the duplex were exposed to the vacuum. ${ }^{12}$ One solution to this problem is to restrain the solute to remain at the center of the solvent and add a boundary 
potential at the surface of the solvent to prevent evaporation. These boundary representations are not recommended unless surface tension effects, which lead to the inhibition of fluctuations at the center of the solvent can be reduced. The imposition of periodic boundary conditions eliminates the vacuum or continuum interface by imposing a lattice structure on the system such that a molecule leaving one side of the periodic box enters the opposite side. By applying periodic boundaries to simulations, the influence, for example, of bulk solvent or crystalline environments can be included, thereby improving the rigor and realism of a model. This condition necessitates the meticulous definition of volume and pressure. Scaling of the box size is used to couple pressure, and velocities are scaled to couple temperature. Together, coupling pressure and temperature, an isobaric/isothermal ensemble (NPT) can be simulated. A drawback to the use of periodic boundary conditions can be the imposition of true periodicity. The influence of periodic images can inhibit motion or neutralize charge effects. ${ }^{107}$ The application of cutoffs to limit the pair interactions to those within the unit cell reduces the negative effects of true periodicity but neglects the effects of long range interactions. ${ }^{117}$

To eliminate the inaccuracy caused by distance based truncation of non-bonded interactions, the particle mesh Ewald (PME) approach can be employed. Traditionally, imposing a cutoff of non-bonded interactions was used to save computational time. The application of the PME method allows simulations of nucleic acids with explicit solvent and counterions in a nanosecond time range. A Gaussian charge distribution of opposite sign is superimposed upon the original point charge, producing a screened charge distribution. The electrostatic interaction between the screened charges is short-ranged. The original distribution is recovered by adding a second Gaussian charge distribution identical to the first, but of opposite sign. The interaction 
between these canceling distributions is B-spline interpolated on a grid calculated in reciprocal or k-space via fast Fourier transforms. The reciprocal space sum is

$$
E_{\text {recip }}=\frac{1}{2 \pi V} \sum_{m \neq 0} \frac{e^{-\pi^{2} m^{2} / k^{2}}}{m^{2}}\left|S(m)^{2}\right|
$$

where $S(m)=\sum_{i=1} q_{i} e^{2 \pi m \cdot r_{i}}, \mathrm{~V}$ denotes the volume, and $\mathrm{m}$ the reciprocal vectors. Periodic boundary conditions are considered the most rigorously correct approach to maintaining proper density and minimizing boundary edge effects, and they can be used with Ewald methods which are currently considered the most accurate for treatment of long-range electrostatic interactions. ${ }^{117}$

There are various simulation protocols; the basic steps are the same, but the ensembles employed can vary, examples include NVE, where the time evolution of a classical system of $N$ particles in a constant volume, $V$, with a constant energy, $E$, is considered. ${ }^{111}$ Other scientifically relevant ensembles include the canonical NVT (constant volume $V$ and constant temperature $T$ ) associated with Helmholtz free energy and the isothermal/isobaric NPT ensemble (constant pressure, $P$, and constant temperature, $T$ ) associated with a Gibbs free energy. Helmholtz free energy is the maximum amount of work a system can do at constant volume and temperature, and Gibbs free energy is the maximum amount of work a system can do at constant pressure.

There are several methods available to keep the temperature of a system constant during a simulation. The simplest method rescales the velocities at each time step by a factor of $\left(T_{0} / T\right)^{1 / 2}$, where $T$ is the current instantaneous temperature and $T_{0}$. is the desired temperature. The Berendsen coupling algorithm ${ }^{118}$ is a refinement of the above velocity rescaling approach. During constant temperature molecular dynamics, the velocities, $v_{i}$, in the system are scaled during each time step of MD. This couples the system, with a temperature relaxation time $\tau_{T}$, to 
a heat bath at $T_{0}$. Velocities are scaled by a factor, $\lambda$, which allows maintaining a desired amount of kinetic energy and is defined as:

$$
\lambda=\left[1+\frac{\Delta t}{2 \tau_{T}}\left(\frac{T_{0}}{T-1}\right)\right]^{\frac{1}{2}}
$$

where $T$ is instantaneous temperature, $\Delta t$ is the size of the integration time step, and $\tau_{T}$ is a characteristic relaxation time. In a manner similar to the velocity scaling, the pressure can also be held constant by rescaling the coordinates of each atom so that a desired pressure is maintained:

$$
\mu=\left[1-\frac{\delta t}{\tau_{P}}\left(P_{0}-P\right)\right]^{\frac{1}{3}}
$$

where $P$ is instantaneous pressure, $\Delta t$ is the size of the integration time step, $\tau_{P}$ is a characteristic relaxation time, and $P_{0}$ is the pressure of the external constant pressure bath. The atomic coordinate, $x$, and the characteristic distance for repeating boundary conditions, $d$, are rescaled to $\mu x$ and $\mu d$, respectively.

The direct outputs of a molecular dynamics simulation are a numerical trajectory file, which contains a series of system snapshots taken at equal time intervals throughout the course of the simulation and a file containing time elapsed reported values of potential, kinetic, and total energy, as well as temperature, pressure, and density for each snapshot. The value of the simulation is dependent upon extracting relevant structural and energetic information from these files. 


\subsection{Free Energy Analysis}

Molecular dynamics simulations provide the means to relate microscopic information such as atomic positions and velocities to macroscopic perceivables such as pressure and energy. For example, the trajectories resulting from a MD simulation can be processed to yield estimates of the relative free energy between states. The approach utilized in this work, MM-PBSA (molecular mechanics Poisson-Boltzman surface area), follows a protocol used extensively to analyze protein conformation. ${ }^{119,120,121,122}$ Molecular dynamics in explicit solvent are used to generate a set of configurations for a given state of interest, such as the B and Z-DNA conformations examined here. Next, the relative free energy between the states is estimated by comparing the average free energies, $\bar{G}$ calculated from all of the configurations included in each state $^{123}$ according to the following equation:

$$
\bar{G}=\bar{E}_{M M}+\bar{G}_{P B S A(G B S A)}-T S_{M M},
$$

where $\bar{E}_{M M}$ is the average molecular mechanical energy, $\bar{G}_{P B S A(G B S A)}$ is the solvation free energy calculated with either a numerical solution to the Poisson-Boltzman equation (PBSA) or by the Generalized Born method (GBSA) and an estimate of the nonpolar free energy with a surface area term, and $-T S_{M M}$ is the negative of the product of temperature and solute entropy. The gas phase energy is calculated for each configuration or in this case each specified frame in the trajectory by removing solvent and counter-ions and calculating the average molecular mechanical energy, $\bar{E}_{M M}$, over the set of specified frames,

$$
\bar{E}_{M M}=\bar{E}_{\text {bond }}+\bar{E}_{\text {angle }}+\bar{E}_{\text {tors }}+\bar{E}_{v d w}+\bar{E}_{\text {elec }}
$$


where these correspond to the bond, angle torsion, Van der Waals, and electrostatic terms in the molecular mechanical force field. This calculation utilizes the same force field as that of the simulation and includes all solute-solute pairwise interactions. ${ }^{123}$ Free energies of solvation are then reintroduced using a continuum solvent model. In this work the Poisson-Boltzman (PB) and the computationally less expensive Generalized Born (GB) model were used to calculate the electrostatic component of free energy for comparison purposes. For the PB approach, calculations were based on the finite difference solution to the Poisson equation: ${ }^{124}$

$$
\nabla \varepsilon(r) \nabla \varphi(r)+4 \pi \rho(r)=0
$$

where $\varphi(r)$ is the electrostatic potential, $\varepsilon(r)$ is the position dependent dielectric function, and $\rho(r)$ is the charge density due to the solute. In the Delphi ${ }^{125}$ program used here, the solute with its associated charges is mapped on to a grid, and the electrostatic potentials due to the presence of a dielectric continuum solvent are determined by solving the Poisson equation. Potentials at the boundaries of the finite-difference lattice are set to sum of the Debye-Huckel potentials as shown in Equation 4.15. ${ }^{126}$

$$
\varphi=\sum_{i} q_{i} \frac{\exp (-\kappa r)}{\varepsilon r}
$$

Here $\varphi$ is the potential estimate at a given lattice boundary point, $q_{i}$ is an atomic partial charge, $r$ is the distance from the lattice boundary to the charge, $\kappa$ is the Debye screening parameter, and $\varepsilon$ is the solvent dielectric constant.

In the second approach, the electrostatics are modeled by the generalized Born model: ${ }^{127}$

$$
\nabla G_{B o r n}^{G B}=\left(1-\frac{1}{\varepsilon}\right) \sum_{i j} \frac{q_{i} q_{j}}{\left({r_{i j}}^{2}+\alpha_{i j}{ }^{2} \exp \left(-D_{i j}\right)\right)^{0.5}},
$$


where $\alpha_{i j}=\left(\alpha_{i} \alpha_{j}\right)^{0.5}, D_{i j}=r_{i j}{ }^{2} / 2 \alpha_{i j}{ }^{2}$, and the double sum runs over all pairs of atoms, including terms where $i=j . \quad \alpha_{\mathrm{i}}$ is the effective Born radius of atom $i$, and the exponential is used to force the term to approximate the dielectric part of the Coulomb's law rapidly as the atoms move beyond the contact distance of their Born radii. The effective Born radius for a simple spherical solute where the charge resides at the center of this sphere (as in a model for a metal ion) can be close to the Van der Waals radius of the solute. However, for more complex solutes, the effective Born radius depends on the positions and volumes of all other solute atoms because they displace the solvent-like dielectric medium. ${ }^{128}$ Physically, the Born radius of an atom is the average distance from the charge on the atom to its dielectric boundary. ${ }^{128}$ In this case the standard pairwise Generalized Born model was used with parameters described by Tsui and Case. $^{129}$

The nonpolar contribution to the solvation free energy uses a solvent accessible surface area dependent term. ${ }^{130}$ This term is represented as $\gamma \mathrm{SA}+b$, where SA is the surface area of the solute, and the constants $\gamma$ and $b$ are $0.00542 \mathrm{kcal} / \AA^{2}$ and $0.92 \mathrm{kcal} / \mathrm{mol}$ respectively. ${ }^{131}$ The solvent-accessible surface area is evaluated using the algorithm of Sanner. ${ }^{132}$ To complete the estimates of the free energy differences between states, the entropy components arising from solute degrees of freedom must also be estimated. In this case, normal mode calculations were carried out using the Nmode program through the MM-PBSA module of Amber. Nmode performs MM calculations, and as applied to this work, uses second and third derivative information to perform vibrational analysis. From this information the rotational, translational, and vibrational components of the entropy term were calculated.

The drawback of this approach to free energy calculations is the lack of explicit sampling pathway between the two states of interest. Without the explicit path, the height of the free 
energy barrier between the states cannot be determined,and the reaction pathway is not fully characterized. ${ }^{123}$ On the other hand, this approach does not require the detailed sampling necessary for full interpretation of the reaction pathway. The methods utilized here depend on the consistent use of the same solute force field in the simulations as in the energy analysis. This approach requires that the two states do not overlap;specifically, the trajectories used for the analysis can not sample the same configurations. ${ }^{123}$ Not only does this approach allow one to compare relative stabilities of nucleic acids and determine the component(s) of free energy that drives the structure in the direction of a preferred conformation, it has also been widely applied to mutagenesis and binding studies of proteins. ${ }^{105,133}$ 


\section{CHAPTER 5}

\section{$5 \quad$ Computational Results}

\section{$5.1 \quad$ Introduction}

Theoretical studies of biological molecules permit the study of relationships between structure, function, and dynamics at the atomic level. Given the number of atoms involved in these systems, treating them using QM is not feasible. Instead, QM is used to generate many of the force field parameters (potential energy function) used to describe the systems. In this case, QM techniques, as well as analogous assignments were used to generate missing force field parameters for the C8-modified oligonucleotides. Once the missing force field parameters were defined, MM was used to calculate the equilibrium geometry of the system, and MD simulations followed. Thermal dynamic properties, as well as dynamic behavior can be studied from the output of the MD simulations.

This chapter describes the force field parameter development for the modified guanine bases, the simulation protocol, and post simulation analysis. Example control files for QM calculations, MD simulations, and free energy analyses are found in the appendices. The system studied in this work is $\mathrm{d}\left(\mathrm{CGCGCG}{ }^{*} \mathrm{CGCG}\right)_{2}$ where $\mathrm{G}^{*}$ is guanine, phenylguanine, $p$ tolylguanine, and $p$-hydroxymethylphenylguanine. From the computational studies coupled with the experimental work previously described, we hope to gain an understanding of the effects of guanine C8 modification on DNA conformation and insight into the mechanism of Z-DNA stabilization of these adducts. 


\subsection{Force Field Development for C8-tolyl, phenyl and hydroxymethyl-guanine}

The parameters for the modified bases were initially developed for C8-p-tolylguanine illustrated in Figure 5.1b. Existing force field atom parameters similar to the atoms present in this modified base were selected (guanine was used for the guanine portion of the modified base, the aromatic carbon and hydrogen types of tyrosine for the tolyl ring, and the methyl group of thymidine for the tolyl methyl group) and served as the starting point for parameter development. A critical parameter, the torsional barrier for rotation about the $\mathrm{C} 8$-aryl bond, was further evaluated using Gaussian98 at the HF/6-31G* level of theory. The initial structure for this was obtained by cutting out the C8-p-tolylguanine from the modified B-DNA. All torsions except the C10-C9-C8-N7 [N9] (Figure 5.1b) were held constant while all bond angles and lengths were

allowed to vary. Seven structures were then generated using HyperChem ${ }^{134}$ by rotating about the C8-p-tolyl bond in 30 degree increments. Each of these structures served as input into the Gaussian calculations. As the tolyl functional group was rotated about the connecting bond in $30^{\circ}$ increments, the energy was calculated at each increment to generate a torsional profile shown in Figure 5.2. The lowest energy conformers occurred at torsion angles of $0^{\circ}$ and $180^{\circ}$ and the highest energy conformer was at $90^{\circ}$ (tolyl ring perpendicular to the plane of the guanine residue). The energy difference between the minimum and maximum was $4.06 \mathrm{kcal} / \mathrm{mol}$. This value (for the C10-C9-C8-N7 [N9] torsion potential [Pk]) was incorporated into the Cornell 95 force field.

Initially, bond lengths, bond angles, stretching constants, and bending constants for the C8-tolyl bond were based on comparisons with similar atoms in the Cornell 95 force field. Small modifications of the bond angles and lengths were made to the starting values using the Gaussian 98 structure generated with a $180^{\circ}$ torsion angle as shown in Figure 5.1b. 
a) C8-phenylguanine,

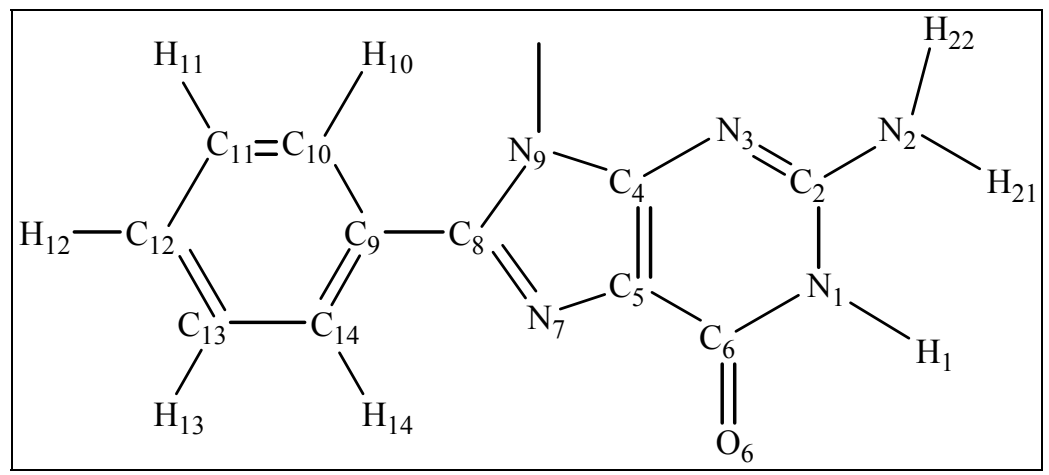

b) C8-p-tolylguanine

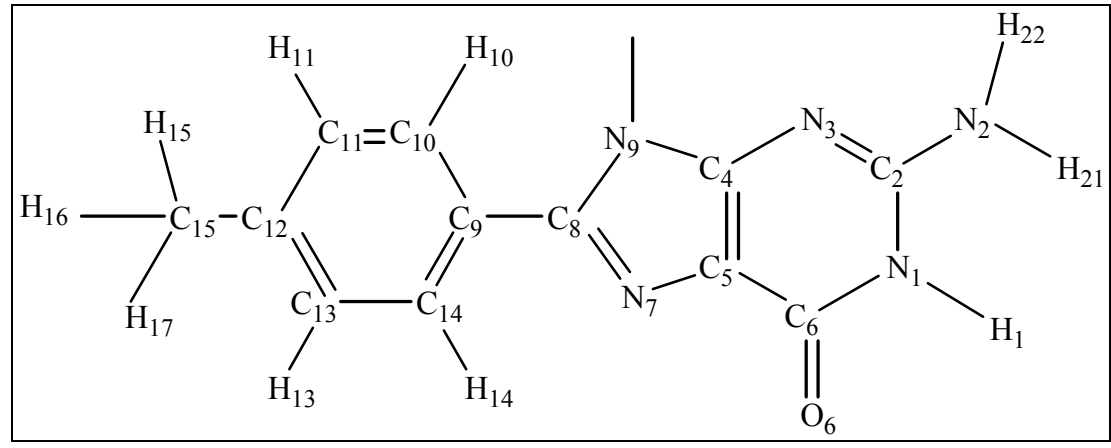

c) C8-p-hydroxymethylphenylguanine

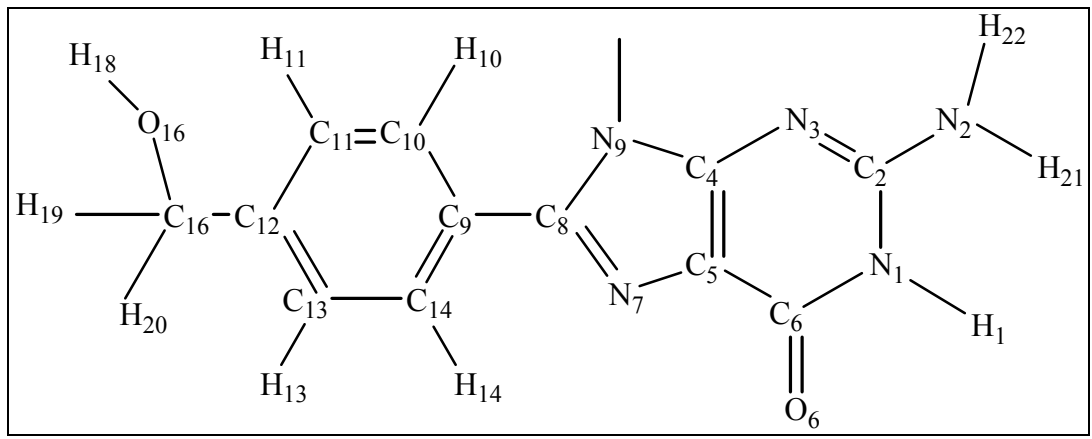

Figure 5.1. Numbering scheme for a) C8-phenylguanine, b) C8-p-tolylguanine, and c) C8-phydroxymethylphenylguanine. 
The stretching and bending constants $\left(\mathrm{k}_{\mathrm{r}}\right.$ and $\left.\mathrm{k}_{\theta}\right)$ for $\mathrm{C} 8-\mathrm{C} 9$ were scaled between those of a C-C single bond and an aromatic $\mathrm{C}-\mathrm{C}$ bond in the Cornell 95 force field, based on the bond lengths and angles calculated by Gaussian98. Next, the value of the electrostatic potential was calculated at a large number of points defined by four shells of surfaces at 1.4, 1.6, 1.8, and 2 times the VDW radii using Gaussian98 (HF/6-31G*, B3YLP). The output from these calculations was used by the Amber utility program RESP to give partial charges for C8-ptolylguanine.

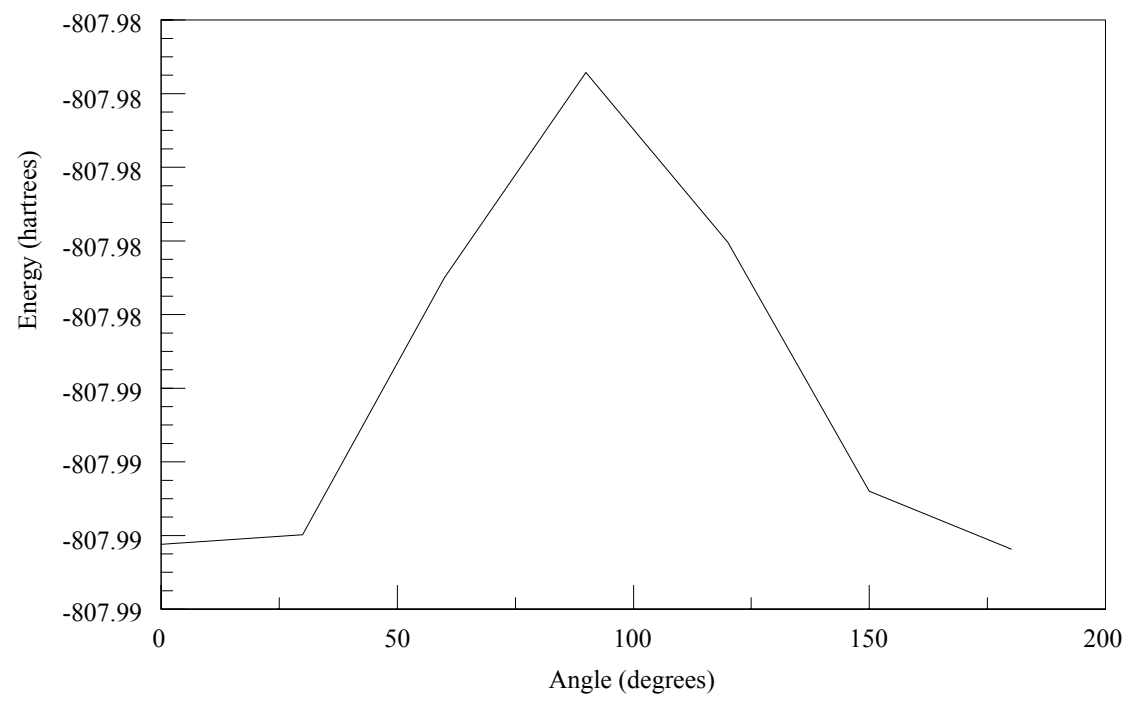

Figure 5.2. Energy profile for rotation about the C8-p-tolylguanine bond.

This completed the best fit parameters for C8-p-tolylguanine with respect to the Cornell 95 force field. To adjust these parameters for C8-phenylguanine (Figure 5.1a), the methyl group of C8-p-tolylguanine was replaced with a hydrogen atom, and the partial charges were derived from performing the above Gaussian calculation and fitting the $6-31 \mathrm{G}^{*}$ electrostatic potential with the RESP algorithm. 
To parameterize C8-hydroxymethylphenylguanine (Figure 5.1c), the Antechamber suite ${ }^{135}$ of Amber $7^{92}$ was utilized. Antechamber is a set of tools to generate "prep" input files for organic molecules. The antechamber program is the main program for Antechamber; it can perform a variety of file conversions, as well as assign atomic charges and atom types. In this case a Gaussian 98 output file was generated as outlined for C8-p-tolylguanine and C8phenylguanine above. The Gaussian file served as input into antechamber. Output requested from antechamber was an Amber prep file and partial charges as calculated by RESP. Next, the parmchk module of Antechamber was used to write a "frcmod" file with the missing parameters. This file contained the missing angle parameters for $\mathrm{O}_{16}-\mathrm{C}_{16}-\mathrm{C}_{12}$. The final parameters for bonds, bond angles, torsions, improper torsions (out-of-plane bending), and atom charges are shown in Tables 5.1-5.5 respectively. Note that only the guanine base parameters were modified. The parameters for 2'-deoxyribose were not modified from those in the Cornell 95 force field. 
Table 5.1. Cornell 95 force field parameters for bonds in C8-arylguanines for use with Amber. Parameters shown in bold type are those developed in this work for the Cornell 95 force field. Parameters shown in parentheses are from crystal structures.

\begin{tabular}{|c|c|c|}
\hline Bond $^{a}$ & $\mathbf{r}_{0}^{b}$ & $\mathbf{k}_{\text {str }}{ }^{\mathrm{c}}$ \\
\hline $\mathrm{N}_{1}-\mathrm{H}_{1}$ & 1.010 & 434 \\
\hline $\mathrm{N}_{2}-\mathrm{H}_{21}$ & 1.010 & 434 \\
\hline $\mathrm{N}_{2}-\mathrm{H}_{22}$ & 1.010 & 434 \\
\hline $\mathrm{C}_{10}-\mathrm{H}_{10}\left(\mathrm{C}_{14}-\mathrm{H}_{14}\right)$ & 1.080 & 367 \\
\hline $\mathrm{C}_{11}-\mathrm{H}_{11}\left(\mathrm{C}_{13}-\mathrm{H}_{13}\right)$ & 1.080 & 367 \\
\hline $\mathrm{C}_{12}-\mathrm{H}_{12}$ & 1.080 & 367 \\
\hline $\mathrm{N}_{1}-\mathrm{C}_{2}$ & 1.381 & 427 \\
\hline $\mathrm{C}_{2}-\mathrm{N}_{2}$ & 1.340 & 481 \\
\hline $\mathrm{C}_{2}-\mathrm{N}_{3}$ & 1.339 & 483 \\
\hline $\mathrm{N}_{3}-\mathrm{C}_{4}$ & 1.354 & 461 \\
\hline $\mathrm{C}_{4}-\mathrm{C}_{5}$ & 1.370 & 520 \\
\hline $\mathrm{C}_{4}-\mathrm{N}_{9}$ & 1.374 & 436 \\
\hline $\mathrm{C}_{5}-\mathrm{C}_{6}$ & 1.419 & 447 \\
\hline $\mathrm{C}_{5}-\mathrm{N}_{7}$ & 1.391 & 414 \\
\hline $\mathrm{C}_{6}-\mathrm{N}_{1}$ & 1.388 & 418 \\
\hline $\mathrm{C}_{6}-\mathrm{O}_{6}$ & 1.229 & 570 \\
\hline $\mathrm{N}_{7}-\mathrm{C}_{8}$ & 1.304 & 529 \\
\hline $\mathrm{C}_{8}-\mathrm{N}_{9}$ & 1.371 & 440 \\
\hline $\mathrm{C}_{8}-\mathrm{C}_{9}$ & 1.470 & 372 \\
\hline $\mathrm{C}_{9}-\mathrm{C}_{10}\left(\mathrm{C}_{9}-\mathrm{C}_{14}\right)$ & $1.400(1.400)$ & 469 \\
\hline $\mathrm{C}_{10}-\mathrm{C}_{11}\left(\mathrm{C}_{14}-\mathrm{C}_{13}\right)$ & $1.400(1.400)$ & 469 \\
\hline $\mathrm{C}_{11}-\mathrm{C}_{12}\left(\mathrm{C}_{13}-\mathrm{C}_{12}\right)$ & $1.400(1.400)$ & 469 \\
\hline $\mathrm{C}_{12}-\mathrm{C}_{15}\left(\mathrm{C}_{12}-\mathrm{C}_{16}\right)$ & $1.510(1.505)$ & 317 \\
\hline $\mathrm{C}_{15}-\mathrm{H}_{15}\left(\mathrm{C}_{15}-\mathrm{H}_{16}\right)\left(\mathrm{C}_{15}-\mathrm{H}_{17}\right)$ & $1.090(0.968)$ & 340 \\
\hline $\mathrm{C}_{16}-\mathrm{H}_{19}\left(\mathrm{C}_{16}-\mathrm{H}_{20}\right)$ & $1.090(0.951)$ & 340 \\
\hline $\mathrm{C}_{16}-\mathrm{O}_{16}$ & $1.410(1.430)$ & 320 \\
\hline
\end{tabular}




\begin{tabular}{|l|l|l|}
\hline $\mathrm{O}_{16}-\mathrm{H}_{18}$ & 0.960 & 553 \\
\hline
\end{tabular}

${ }^{\mathrm{a}}$ Atom numbers refer to the numbering schemes in Figures 5.1 and 5.3. ${ }^{\mathrm{b}}$ Equilibrium bond lengths, in angstroms. ${ }^{\mathrm{c} B o n d}$ stretching constants in $\mathrm{kcal} / \mathrm{mole}$-angstrom.

Table 5.2. Cornell 95 force field parameters for bond angles in C8-arylguanines for use with Amber. Parameters shown in parentheses are from crystal structures.

\begin{tabular}{|c|c|c|}
\hline Bond Angle $^{a}$ & $\theta_{0}^{b}$ & $\mathbf{k}_{\text {bend }}{ }^{c}$ \\
\hline $\mathrm{H}_{1}-\mathrm{N}_{1}-\mathrm{C}_{2}$ & 120.0 & 30 \\
\hline $\mathrm{H}_{21}-\mathrm{N}_{2}-\mathrm{C}_{2}$ & 120.0 & 35 \\
\hline $\mathrm{H}_{22}-\mathrm{N}_{2}-\mathrm{C}_{2}$ & 120.0 & 35 \\
\hline $\mathrm{H}_{1}-\mathrm{N}_{1}-\mathrm{C}_{6}$ & 116.8 & 30 \\
\hline $\mathrm{H}_{10}-\mathrm{C}_{10}-\mathrm{C}_{9}\left(\mathrm{H}_{14}-\mathrm{C}_{14}-\mathrm{C}_{9}\right)$ & 120.0 & 35 \\
\hline $\mathrm{H}_{10}-\mathrm{C}_{10}-\mathrm{C}_{11}\left(\mathrm{H}_{14}-\mathrm{C}_{14}-\mathrm{C}_{13}\right)$ & 120.0 & 35 \\
\hline $\mathrm{H}_{11}-\mathrm{C}_{11}-\mathrm{C}_{10}\left(\mathrm{H}_{13}-\mathrm{C}_{13}-\mathrm{C}_{14}\right)$ & 120.0 & 35 \\
\hline $\mathrm{H}_{11}-\mathrm{C}_{11}-\mathrm{C}_{12}\left(\mathrm{H}_{13}-\mathrm{C}_{13}-\mathrm{C}_{12}\right)$ & 120.0 & 35 \\
\hline $\mathrm{H}_{12}-\mathrm{C}_{12}-\mathrm{C}_{11}\left(\mathrm{H}_{12}-\mathrm{C}_{12}-\mathrm{C}_{13}\right)$ & 120.0 & 35 \\
\hline $\mathrm{N}_{1}-\mathrm{C}_{2}-\mathrm{N}_{3}$ & 123.3 & 70 \\
\hline $\mathrm{N}_{1}-\mathrm{C}_{2}-\mathrm{N}_{2}$ & 116.0 & 70 \\
\hline $\mathrm{C}_{2}-\mathrm{N}_{3}-\mathrm{C}_{4}$ & 112.0 & 70 \\
\hline $\mathrm{N}_{3}-\mathrm{C}_{2}-\mathrm{N}_{2}$ & 119.3 & 70 \\
\hline $\mathrm{N}_{3}-\mathrm{C}_{4}-\mathrm{C}_{5}$ & 127.7 & 70 \\
\hline $\mathrm{N}_{3}-\mathrm{C}_{4}-\mathrm{N}_{9}$ & 126.2 & 70 \\
\hline $\mathrm{C}_{4}-\mathrm{C}_{5}-\mathrm{C}_{6}$ & 119.2 & 63 \\
\hline $\mathrm{C}_{4}-\mathrm{C}_{5}-\mathrm{N}_{7}$ & 110.4 & 70 \\
\hline $\mathrm{C}_{4}-\mathrm{N}_{9}-\mathrm{C}_{8}$ & 105.4 & 70 \\
\hline $\mathrm{C}_{5}-\mathrm{C}_{6}-\mathrm{N}_{1}$ & 111.3 & 70 \\
\hline $\mathrm{C}_{5}-\mathrm{C}_{6}-\mathrm{O}_{6}$ & 128.8 & 80 \\
\hline $\mathrm{C}_{6}-\mathrm{C}_{5}-\mathrm{N}_{7}$ & 130.0 & 70 \\
\hline $\mathrm{C}_{5}-\mathrm{N}_{7}-\mathrm{C}_{8}$ & 103.8 & 70 \\
\hline $0_{6}-\mathrm{C}_{6}-\mathrm{N}_{7}$ & 120.6 & 80 \\
\hline
\end{tabular}




\begin{tabular}{|l|l|l|}
\hline $\mathbf{N}_{7}-\mathbf{C}_{\mathbf{8}}-\mathbf{C}_{\mathbf{9}}$ & $\mathbf{1 2 5 . 1 5}$ & $\mathbf{7 0}$ \\
\hline $\mathrm{N}_{7}-\mathrm{C}_{8}-\mathrm{N}_{9}$ & 113.9 & 70 \\
\hline $\mathbf{C}_{\mathbf{8}}-\mathbf{C}_{\mathbf{9}}-\mathbf{C}_{\mathbf{1 0}}\left(\mathbf{C}_{\mathbf{8}}-\mathbf{C}_{\mathbf{9}}-\mathbf{C}_{\mathbf{1 4}}\right)$ & $\mathbf{1 1 9 . 0}$ & $\mathbf{7 0}$ \\
\hline $\mathbf{N}_{\mathbf{9}}-\mathbf{C}_{\mathbf{8}}-\mathbf{C}_{\mathbf{9}}$ & $\mathbf{1 2 3 . 2 4}$ & $\mathbf{7 0}$ \\
\hline $\mathrm{C}_{9}-\mathrm{C}_{10}-\mathrm{C}_{11}\left(\mathrm{C}_{9}-\mathrm{C}_{14}-\mathrm{C}_{13}\right)$ & $120.0(120.0)$ & 63 \\
\hline $\mathrm{C}_{10}-\mathrm{C}_{11}-\mathrm{C}_{12}\left(\mathrm{C}_{14}-\mathrm{C}_{13}-\mathrm{C}_{12}\right)$ & $120.0(120.0)$ & 63 \\
\hline $\mathrm{C}_{11}-\mathrm{C}_{12}-\mathrm{C}_{13}$ & $120.0(120.0)$ & 63 \\
\hline $\mathrm{C}_{11}-\mathrm{C}_{12}-\mathrm{C}_{15}\left(\mathrm{C}_{13}-\mathrm{C}_{12}-\mathrm{C}_{15}\right)$ & $120.0(120.0)$ & 70 \\
\hline $\mathrm{C}_{12}-\mathrm{C}_{15}-\mathrm{H}_{15}\left(\mathrm{C}_{12}-\mathrm{C}_{15}-\mathrm{H}_{16} / \mathrm{H}_{17}\right)$ & $109.5(107.3)$ & 50 \\
\hline $\mathrm{C}_{11}-\mathrm{C}_{12}-\mathrm{C}_{16}\left(\mathrm{C}_{13}-\mathrm{C}_{12}-\mathrm{C}_{16}\right)$ & $120.0(118.1)$ & 70 \\
\hline $\mathbf{C}_{12}-\mathbf{C}_{16}-\mathbf{O}_{\mathbf{1 6}}$ & $\mathbf{1 1 0 . 7 2}$ & $\mathbf{6 7 . 1}$ \\
\hline $\mathbf{C}_{\mathbf{1 2}}-\mathbf{C}_{\mathbf{1 6}}-\mathrm{H}_{\mathbf{2 0}}\left(\mathbf{C}_{\mathbf{1 2}}-\mathrm{C}_{\mathbf{1 6}}-\mathrm{H}_{\mathbf{1 9}}\right)$ & $\mathbf{1 0 9 . 5}$ & $\mathbf{5 0}$ \\
\hline $\mathrm{C}_{16}-\mathrm{O}_{16}-\mathrm{H}_{18}$ & 108.5 & 55 \\
\hline $\mathrm{O}_{16}-\mathrm{C}_{16}-\mathrm{H}_{19}\left(\mathrm{O}_{16}-\mathrm{C}_{16}-\mathrm{H}_{20}\right)$ & 109.5 & 50 \\
\hline
\end{tabular}

${ }^{a}$ Atom numbers refer to the numbering schemes in Figures 5.1 and 5.3. ${ }^{b}$ Equilibrium bond angles in degrees. ${ }^{\mathrm{c}}$ Bending constants in $\mathrm{kcal} / \mathrm{mole}$-angstrom ${ }^{2}$.

Table 5.3. Cornell 95 force field parameters for bond torsions in C8-arylguanines for use with Amber. Parameters shown in parentheses are from crystal structures.

\begin{tabular}{|l|l|l|l|l|}
\hline Bond $^{\mathbf{a}}$ & IDIVF $^{\mathbf{b}}$ & PHASE $^{\mathbf{d}}$ & PK $^{\mathbf{c}}$ & PN $^{\mathbf{e}}$ \\
\hline $\mathrm{H}_{1}-\mathrm{N}_{1}-\mathrm{C}_{2}-\mathrm{N}_{2}$ & 4 & 6 & 180 & 2 \\
\hline $\mathrm{H}_{1}-\mathrm{N}_{1}-\mathrm{C}_{2}-\mathrm{N}_{3}$ & 4 & 6 & 180 & 2 \\
\hline $\mathrm{H}_{21}-\mathrm{N}_{2}-\mathrm{C}_{2}-\mathrm{N}_{3}$ & 4 & 9.6 & 180 & 2 \\
\hline $\mathrm{H}_{22}-\mathrm{N}_{2}-\mathrm{C}_{2}-\mathrm{N}_{3}$ & 4 & 9.6 & 180 & 2 \\
\hline $\mathrm{H}_{10}-\mathrm{C}_{10}-\mathrm{C}_{11}-\mathrm{H}_{11}\left(\mathrm{H}_{14}-\mathrm{C}_{14}-\mathrm{C}_{13}-\mathrm{H}_{13}\right)$ & 4 & 14.5 & 180 & 2 \\
\hline $\mathrm{H}_{10}-\mathrm{C}_{10}-\mathrm{C}_{9}-\mathrm{C}_{8}\left(\mathrm{H}_{14}-\mathrm{C}_{14}-\mathrm{C}_{9}-\mathrm{C}_{8}\right)$ & 4 & 6 & 180 & 2 \\
\hline $\mathrm{H}_{10}-\mathrm{C}_{10}-\mathrm{C}_{9}-\mathrm{C}_{14}\left(\mathrm{H}_{14}-\mathrm{C}_{14}-\mathrm{C}_{9}-\mathrm{C}_{10}\right)$ & 4 & 6 & 180 & 2 \\
\hline $\mathrm{H}_{11}-\mathrm{C}_{11}-\mathrm{C}_{10}-\mathrm{C}_{9}\left(\mathrm{H}_{13}-\mathrm{C}_{13}-\mathrm{C}_{14}-\mathrm{C}_{9}\right)$ & 4 & 6 & 180 & 2 \\
\hline $\mathrm{H}_{11}-\mathrm{C}_{11}-\mathrm{C}_{12}-\mathrm{H}_{12}\left(\mathrm{H}_{13}-\mathrm{C}_{13}-\mathrm{C}_{12}-\mathrm{H}_{12}\right)$ & 4 & 6 & 180 & 2 \\
\hline $\mathrm{H}_{11}-\mathrm{C}_{11}-\mathrm{C}_{12}-\mathrm{C}_{13}\left(\mathrm{H}_{13}-\mathrm{C}_{13}-\mathrm{C}_{12}-\mathrm{C}_{11}\right)$ & 4 & 6 & 180 & 2 \\
\hline
\end{tabular}




\begin{tabular}{|c|c|c|c|c|}
\hline $\mathrm{H}_{12}-\mathrm{C}_{12}-\mathrm{C}_{11}-\mathrm{C}_{10}\left(\mathrm{H}_{12}-\mathrm{C}_{12}-\mathrm{C}_{13}-\mathrm{C}_{14}\right)$ & 4 & 6 & 180 & 2 \\
\hline $\mathrm{N}_{1}-\mathrm{C}_{2}-\mathrm{N}_{3}-\mathrm{C}_{4}$ & 2 & 9.6 & 180 & 2 \\
\hline $\mathrm{C}_{2}-\mathrm{N}_{3}-\mathrm{C}_{4}-\mathrm{C}_{5}$ & 2 & 8.3 & 180 & 2 \\
\hline $\mathrm{C}_{2}-\mathrm{N}_{3}-\mathrm{C}_{4}-\mathrm{N}_{9}$ & 2 & 8.3 & 180 & 2 \\
\hline $\mathrm{N}_{3}-\mathrm{C}_{4}-\mathrm{C}_{5}-\mathrm{C}_{6}$ & 4 & 21.8 & 180 & 2 \\
\hline $\mathrm{N}_{3}-\mathrm{C}_{4}-\mathrm{C}_{5}-\mathrm{N}_{7}$ & 4 & 21.8 & 180 & 2 \\
\hline $\mathrm{N}_{3}-\mathrm{C}_{4}-\mathrm{N}_{9}-\mathrm{C}_{8}$ & 4 & 6.6 & 180 & 2 \\
\hline $\mathrm{C}_{4}-\mathrm{C}_{5}-\mathrm{C}_{6}-\mathrm{O}_{6}$ & 4 & 12 & 180 & 2 \\
\hline $\mathrm{C}_{4}-\mathrm{C}_{5}-\mathrm{C}_{6}-\mathrm{N}_{1}$ & 4 & 12 & 180 & 2 \\
\hline $\mathrm{C}_{4}-\mathrm{C}_{5}-\mathrm{N}_{7}-\mathrm{C}_{8}$ & 2 & 5.1 & 180 & 2 \\
\hline $\mathrm{C}_{4}-\mathrm{N}_{9}-\mathrm{C}_{8}-\mathrm{N}_{7}$ & 4 & 6.8 & 180 & 2 \\
\hline $\mathrm{C}_{4}-\mathrm{N}_{9}-\mathrm{C}_{8}-\mathrm{C}_{9}$ & 4 & 6.8 & 180 & 2 \\
\hline $\mathrm{C}_{5}-\mathrm{C}_{6}-\mathrm{N}_{1}-\mathrm{C}_{2}$ & 4 & 5.4 & 180 & 2 \\
\hline $\mathrm{C}_{5}-\mathrm{N}_{7}-\mathrm{C}_{8}-\mathrm{C}_{9}$ & 2 & 20 & 180 & 2 \\
\hline $\mathrm{C}_{5}-\mathrm{N}_{7}-\mathrm{C}_{8}-\mathrm{N}_{9}$ & 2 & 20 & 180 & 2 \\
\hline $\mathrm{C}_{6}-\mathrm{N}_{1}-\mathrm{C}_{2}-\mathrm{N}_{3}$ & 4 & 6 & 180 & 2 \\
\hline $\mathrm{N}_{7}-\mathrm{C}_{8}-\mathrm{C}_{9}-\mathrm{C}_{10}\left(\mathrm{~N}_{7}-\mathrm{C}_{8}-\mathrm{C}_{9}-\mathrm{C}_{14}\right)$ & 4 & 2.03 & 180 & 2 \\
\hline $\mathrm{C}_{8}-\mathrm{C}_{9}-\mathrm{C}_{10}-\mathrm{C}_{11}\left(\mathrm{C}_{8}-\mathrm{C}_{9}-\mathrm{C}_{14}-\mathrm{C}_{13}\right)$ & 4 & 14.5 & 180 & 2 \\
\hline $\mathrm{N}_{9}-\mathrm{C}_{8}-\mathrm{C}_{9}-\mathrm{C}_{10}\left(\mathrm{~N}_{9}-\mathrm{C}_{8}-\mathrm{C}_{9}-\mathrm{C}_{14}\right)$ & 4 & 2.03 & 180 & 2 \\
\hline $\mathrm{C}_{9}-\mathrm{C}_{10}-\mathrm{C}_{11}-\mathrm{C}_{12}\left(\mathrm{C}_{9}-\mathrm{C}_{14}-\mathrm{C}_{13}-\mathrm{C}_{12}\right)$ & 4 & 14.5 & $180(0.0)$ & 2 \\
\hline $\mathrm{C}_{10}-\mathrm{C}_{11}-\mathrm{C}_{12}-\mathrm{C}_{13}\left(\mathrm{C}_{14}-\mathrm{C}_{13}-\mathrm{C}_{12}-\mathrm{C}_{11}\right)$ & 4 & 14.5 & $180(0.0)$ & 2 \\
\hline $\mathrm{C}_{11}-\mathrm{C}_{12}-\mathrm{C}_{13}-\mathrm{C}_{14}\left(\mathrm{C}_{13}-\mathrm{C}_{12}-\mathrm{C}_{11}-\mathrm{C}_{10}\right)$ & 4 & 14.5 & $180(0.0)$ & 2 \\
\hline $\mathrm{H}_{11}-\mathrm{C}_{11}-\mathrm{C}_{12}-\mathrm{C}_{15}\left(\mathrm{H}_{13}-\mathrm{C}_{11}-\mathrm{C}_{12}-\mathrm{C}_{15}\right)$ & 4 & 14.5 & $180(0.0)$ & 2 \\
\hline $\mathrm{C}_{11}-\mathrm{C}_{12}-\mathrm{C}_{15}-\mathrm{H}_{15}\left(\mathrm{C}_{11}-\mathrm{C}_{12}-\mathrm{C}_{15}-\mathrm{H}_{16} / \mathrm{H}_{17}\right)$ & 6 & 0.0 & 0.0 & 2 \\
\hline $\mathrm{H}_{11}-\mathrm{C}_{11}-\mathrm{C}_{12}-\mathrm{C}_{16}$ & 4 & 14.5 & 180 & 2 \\
\hline $\mathrm{C}_{11}-\mathrm{C}_{12}-\mathrm{C}_{16}-\mathrm{O}_{16}$ & 6 & 0.0 & $0.0(92.1)$ & 2 \\
\hline $\mathrm{C}_{11}-\mathrm{C}_{12}-\mathrm{C}_{16}-\mathrm{H}_{19}\left(\mathrm{C}_{11}-\mathrm{C}_{12}-\mathrm{C}_{16}-\mathrm{H}_{20}\right)$ & 6 & 0.0 & 0.0 & 2 \\
\hline $\mathrm{C}_{12}-\mathrm{C}_{16}-\mathrm{O}_{16}-\mathrm{H}_{18}$ & 3 & 0.5 & 0.0 & 3 \\
\hline
\end{tabular}


${ }^{\mathrm{a}}$ Atom numbers refer to the numbering schemes in Figures 5.1 and 5.3. ${ }^{\mathrm{b}}$ IDIVF was determinined as described in the Amber 6 manual. ${ }^{\mathrm{c}} \mathrm{PK}$ is the potenial of the torsion in kcal $/ \mathrm{mole}$. ${ }^{\mathrm{d}} \mathrm{Phase}$ is the phase shift required to properly reproduce the barriers by the truncated Fourier series. ${ }^{\mathrm{e}} \mathrm{PN}$ corresponds to the number of maxima seen in the energy profile.

Table 5.4. Cornell 95 force field parameters for improper bond torsions in C8-arylguanines for use with Amber.

\begin{tabular}{|l|l|}
\hline Bond & PK $^{\mathbf{b}}$ \\
\hline LP-C $-\mathrm{N}_{3}-\mathrm{C}_{4}$ & 1.0 \\
\hline LP- $\mathrm{H}_{21}-\mathrm{N}_{2}-\mathrm{H}_{22}$ & 1.0 \\
\hline $\mathrm{LP}-\mathrm{C}_{5}-\mathrm{N}_{7}-\mathrm{C}_{8}$ & 1.0 \\
\hline $\mathrm{H}_{1}-\mathrm{C}_{6}-\mathrm{N}_{1}-\mathrm{C}_{2}$ & 1.0 \\
\hline $\mathrm{H}_{21}-\mathrm{H}_{22}-\mathrm{N}_{2}-\mathrm{C}_{2}\left(\mathrm{H}_{22}-\mathrm{H}_{21}-\mathrm{N}_{2}-\mathrm{C}_{2}\right)$ & 1.0 \\
\hline $\mathrm{H}_{10}-\mathrm{C}_{9}-\mathrm{C}_{10}-\mathrm{C}_{11}\left(\mathrm{H}_{14}-\mathrm{C}_{9}-\mathrm{C}_{14}-\mathrm{C}_{13}\right)$ & 1.1 \\
\hline $\mathrm{H}_{11}-\mathrm{C}_{10}-\mathrm{C}_{11}-\mathrm{C}_{12}\left(\mathrm{H}_{13}-\mathrm{C}_{14}-\mathrm{C}_{13}-\mathrm{C}_{12}\right)$ & 1.1 \\
\hline $\mathrm{H}_{12}-\mathrm{C}_{11}-\mathrm{C}_{12}-\mathrm{C}_{13}$ & 1.1 \\
\hline $\mathrm{N}_{1}-\mathrm{N}_{2}-\mathrm{C}_{2}-\mathrm{N}_{3}$ & 10.5 \\
\hline $\mathrm{N}_{3}-\mathrm{C}_{5}-\mathrm{C}_{4}-\mathrm{N}_{9}$ & 1.1 \\
\hline $\mathrm{C}_{4}-\mathrm{C}_{6}-\mathrm{C}_{5}-\mathrm{N}_{7}$ & 1.1 \\
\hline $\mathrm{N}_{1}-\mathrm{C}_{5}-\mathrm{C}_{6}-\mathrm{O}_{6}$ & 10.5 \\
\hline $\mathrm{N}_{7}-\mathrm{N}_{9}-\mathrm{C}_{8}-\mathrm{C}_{9}$ & 1.1 \\
\hline $\mathrm{C}_{4}-\mathrm{C}_{1}-\mathrm{N}_{9}-\mathrm{C}_{8}$ & 1.0 \\
\hline $\mathrm{C}_{8}-\mathrm{C}_{10}-\mathrm{C}_{9}-\mathrm{C}_{14}$ & 1.1 \\
\hline $\mathrm{C}_{11}-\mathrm{C}_{13}-\mathrm{C}_{12}-\mathrm{C}_{15}\left(\mathrm{C}_{11}-\mathrm{C}_{13}-\mathrm{C}_{12}-\mathrm{C}_{16}\right)$ & 1.1 \\
\hline
\end{tabular}

${ }^{a}$ Atom numbers refer to the numbering schemes in Figures 5.1 and 5.3. For improper torsions, the third atom listed is the central atom; the remaining three atoms are attached to the central atom. Improper torsions are not required input. ${ }^{\mathrm{b}}$ Values of PK are in $\mathrm{kcal} / \mathrm{mole}$. 
Table 5.5. Partial electronic charges of C8-arylguanines evaluated by fitting the HF6-31G* electrostatic potentials with the RESP algorithm.

\begin{tabular}{|c|c|c|c|}
\hline Atom & Charge $C G^{8-P h g}$ & Charge $C G^{8-T o l}$ & Charge $C G^{8-H m p}$ \\
\hline N9 & 0.0577 & 0.0577 & 0.0577 \\
\hline $\mathrm{C} 8$ & 0.0736 & 0.0736 & 0.0736 \\
\hline N7 & -0.5725 & -0.5725 & -0.5725 \\
\hline $\mathrm{C} 5$ & 0.1991 & 0.1991 & 0.1991 \\
\hline $\mathrm{C} 6$ & 0.4918 & 0.4918 & 0.4918 \\
\hline O6 & -0.5699 & -0.5699 & -0.5699 \\
\hline N1 & -0.5053 & -0.5053 & -0.5053 \\
\hline $\mathrm{H} 1$ & 0.3520 & 0.3520 & 0.3520 \\
\hline $\mathrm{C} 2$ & 0.7432 & 0.7432 & 0.7432 \\
\hline N2 & -0.9230 & -0.9230 & -0.9230 \\
\hline $\mathrm{H} 21$ & 0.4235 & 0.4235 & 0.4235 \\
\hline $\mathrm{H} 22$ & 0.4235 & 0.4235 & 0.4235 \\
\hline N3 & -0.6636 & -0.6636 & -0.6636 \\
\hline $\mathrm{C} 4$ & 0.1814 & 0.1814 & 0.1814 \\
\hline C9 & 0.0747 & 0.0747 & 0.0747 \\
\hline $\mathrm{C10}$ & -0.2509 & -0.2509 & -0.2509 \\
\hline C11 & -0.1706 & -0.1706 & -0.1706 \\
\hline $\mathrm{C} 12$ & 0.1217 & 0.1217 & 0.1217 \\
\hline C13 & -0.2060 & -0.2060 & -0.2060 \\
\hline C14 & -0.0828 & -0.0828 & -0.0828 \\
\hline H10 & 0.1481 & 0.1481 & 0.1481 \\
\hline H11 & 0.1431 & 0.1431 & 0.1420 \\
\hline H12 & 0.1550 & ------- ${ }^{a}$ & -------- \\
\hline H13 & 0.1457 & 0.1476 & 0.1477 \\
\hline H14 & 0.1173 & 0.1173 & 0.1190 \\
\hline C15 & $\begin{array}{l}-------- \\
\end{array}$ & -0.2217 & ------- \\
\hline H15 & -------- & 0.0760 & -------- \\
\hline H16 & --------- & 0.0760 & -------- \\
\hline H17 & --------- & 0.0760 & -------- \\
\hline C16 & -------- & --------- & $\mathbf{0 . 2 2 1 7}$ \\
\hline 016 & --------- & --------- & -0.5960 \\
\hline H18 & --------- & --------- & $\mathbf{0 . 3 8 0 0}$ \\
\hline H19 & --------- & --------- & $\mathbf{0 . 0 3 5 0}$ \\
\hline $\mathbf{H 2 0}$ & --------- & --------- & $\mathbf{0 . 0 3 5 0}$ \\
\hline
\end{tabular}

${ }^{a}$ Atom not present in compound.

Lack of x-ray data makes the new force field parameters difficult to access. Fortunately, crystal structures are available for benzene (Cambridge Structural Database [CSD] entry BENZEN01), toluene (CSD entry TOLUEN), and iodobenzylalcohol. ${ }^{136}$ Using MOIL-view to 
measure bond lengths, angles, and torsions, good agreement was found between the crystal structures (data shown in parentheses in Tables 5.1, 5.2, and 5.3) and the current force field parameters for the arylguanines. The structures and numbering scheme for benzene, toluene, and iodobenzylalcohol are shown in Figure 5.3 below. We were unable to assess the C9-C8-N7 (N9) bond angles and C10-C9-C8-N7 (N9) torsion angles from crystal data.

a) Benzene

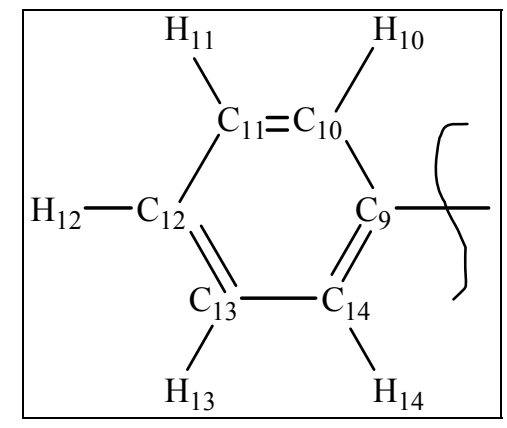

b) Toluene

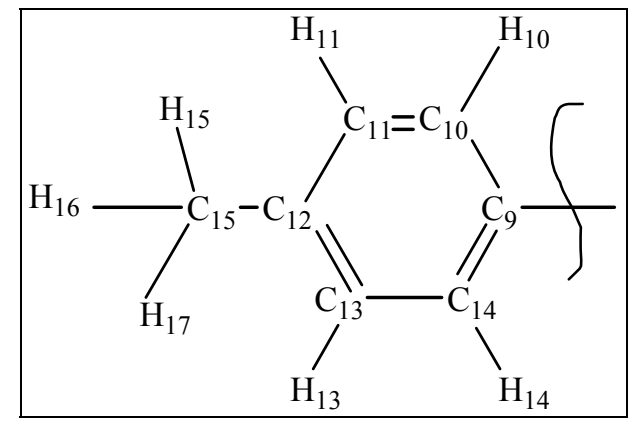

c) Benzyliodoalcohol

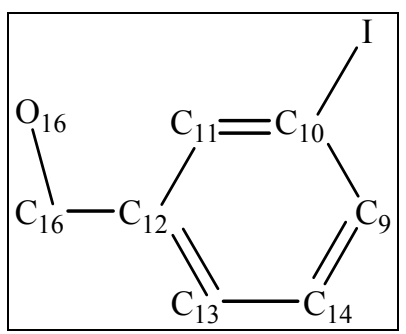

Figure 5.3. Numbering scheme for a) benzene, b) toluene, and c) iodobenzylalcohol. 


\subsection{Simulation Protocol}

Molecular modeling and dynamics were performed with Amber and the Cornell 95 force field. All oligonucleotide structures were built using Sybyl (Tripos, Inc.) and then transferred to Amber 6. The unmodified sequences (B and Z) were ${ }^{5} \mathrm{CGCGCGCGCG}^{3^{\prime}}$ (CG). The modified sequences were constructed from the unmodified sequences by attaching a phenyl, tolyl, or hydroxymethylphenyl group on the C8-position of G6 and G16. Hydrogen atoms, 18 sodium counterions to neutralize the charge, and a water box were added within the LEaP module of Amber. The ions are added in a shell around the DNAs using a Coulombic potential on a grid. For nucleic acids with "regular" backbones the first cations are generally placed between phosphates and the final two cations are positioned around the center of the molecule. ${ }^{92}$ In this case the TIP3P water model ${ }^{116}$ was used and a buffer zone of 10 angstroms between the closest atom in the solute and the wall of the solvent box was specified. Water box size varied as a function of the size of the aryl group added to G6 and G16 and DNA type (B or Z). Box dimensions and the number of water residues added to each sequence are given in Table 5.6. Initially, the water and solute were equilibrated by minimizing the water and counterions with the DNA fixed to a gradient convergence of $10^{-3} \mathrm{kcal} / \mathrm{mol} \cdot \AA$, with $500 \mathrm{kcal} / \mathrm{mol} \cdot \AA$ positional restraints on nucleic acids. This was followed by 25 ps of non-particle mesh Ewald (PME) dynamics with the DNA fixed to allow for equilibration of water and counterions. 
Table 5.6. Box dimensions and number of solvent residues for each system.

\begin{tabular}{|l|l|l|}
\hline Sequence & $\begin{array}{l}\text { Box } \\
\text { Dimensions }\end{array}$ & $\begin{array}{l}\text { Number of Solvent } \\
\text { Residues }\end{array}$ \\
\hline $\mathbf{C G}$ & $45 \times 47 \times 63$ & 3102 \\
\hline $\mathbf{C G}^{\text {8-Ph }}$ & $45 \times 47 \times 63$ & 3139 \\
\hline $\mathbf{C G}^{\text {8-Tol }}$ & $46 \times 47 \times 63$ & 3209 \\
\hline $\mathbf{C G}^{\text {8-Hmp }}$ & $46 \times 47 \times 63$ & 3232 \\
\hline $\mathbf{C G}(\mathbf{Z})$ & $43 \times 43 \times 65$ & 2663 \\
\hline $\mathbf{C G}^{\text {-Ph }}(\mathbf{Z})$ & $44 \times 48 \times 65$ & 3183 \\
\hline $\mathbf{C G}^{\text {-Tol }}(\mathbf{Z})$ & $44 \times 50 \times 65$ & 3356 \\
\hline $\mathbf{C G}^{\text {8-Hmp }}(\mathbf{Z})$ & $45 \times 50 \times 65$ & 3405 \\
\hline
\end{tabular}

All subsequent MD simulations were performed in the isothermal-isobaric ensemble (300 $\mathrm{K}, 1 \mathrm{~atm}$ ) using periodic boundary conditions. The SHAKE algorithm was applied to hydrogen atoms with a tolerance limit of $0.0005 \AA$ and a 2 femtosecond (fs) timestep. SHAKE is used to extend time steps, allowing for longer simulations by removing the fastest motions, i.e., the $\mathrm{X}-\mathrm{H}$ stretching freedom which has no real consequence on the result. Time step $(\delta t)$ determination is dependent upon the period of fastest motion $(\tau)$; a good rule of thumb is $\frac{\delta t}{\tau}=20 .{ }^{111}$ A $9 \AA$ cutoff was applied to Lennard-Jones interactions. Simulations were performed using Berendsen temperature coupling algorithm and constant pressure with isotropic molecule based scaling (each with a 0.2 ps time constant). By this method, each DNA strand is treated as a different molecule, and shifting of these strands is avoided during equilibration. The non-bonded pair list was updated every $20 \mathrm{fs}$, and the Particle mesh-Ewald method (PME), for inclusion of longrange electrostatic interactions without truncation, was used.

The next step in the simulation protocol was 25 ps of PME dynamics for inclusion of long-range electrostatic interactions without truncation. This method is a fast method for performing Ewald summation of Coulombic interactions. It means that the system is treated as if it was truly periodic and includes all electrostatic interactions within the unit cell (up to the cut- 
off) and with all image cells. The PME charge grid spacing was approximately $1.0 \AA$, and the charge grid was interpolated on a cubic grid with the direct sum tolerance set to $10^{-4}$.

Following the 25 ps PME dynammics, the entire structure was minimized for 1000 steps with $25 \mathrm{kcal} / \mathrm{mole} \cdot \AA$ of positional restrains on DNA followed by 3 ps dynamics run, which allowed water and $\mathrm{Na}^{+}$ions to relax around the solute. Subsequently, 600 steps of equilibration with gradual removal of positional restraints by $5 \mathrm{kcal} / \mathrm{mole} \cdot \AA$ on the solute were performed. During the final dynamics, the system was heated from $100 \mathrm{~K}$ to $300 \mathrm{~K}$ over $20 \mathrm{ps.} \mathrm{After} \mathrm{each}$ dynamics simulation, the PME box information was updated to match the final box coordinates from previous runs. Production runs of approximately 2 ns duration, at constant temperature/constant pressure $(\mathrm{T}=300 \mathrm{~K} / \mathrm{P}=1 \mathrm{~atm})$, were performed after the final equilibration step. The resulting trajectories were analyzed using CURVES 5.0, ${ }^{137,138}$ the ptraj module of Amber 6, Dials and Windows, ${ }^{137,139}$ and MOIL-view. ${ }^{140}$ 


\subsection{Results}

\subsubsection{Trajectory Stability}

Figures 5.4 through 5.11 depict the pressure, temperature, density, and energies over the course of the production run for the unmodified, as well as the phenyl and tolyl-modified DNA's in the $\mathrm{B}$ and $\mathrm{Z}$ conformations. During the production run, pressure and temperature were kept constant at $1 \mathrm{~atm}$ and $300 \mathrm{~K}$, respectively. Examination of the pressure variable reveals seemingly large fluctuations in instantaneous pressure. This phenomenon is to be expected, ${ }^{92}$ and in all cases the average value of pressure over the course of the trajectory was within 1 atm of the target pressure of $1 \mathrm{~atm}$. Overall, these figures demonstrate stabilized conditions over the course of the trajectories for all systems examined. 


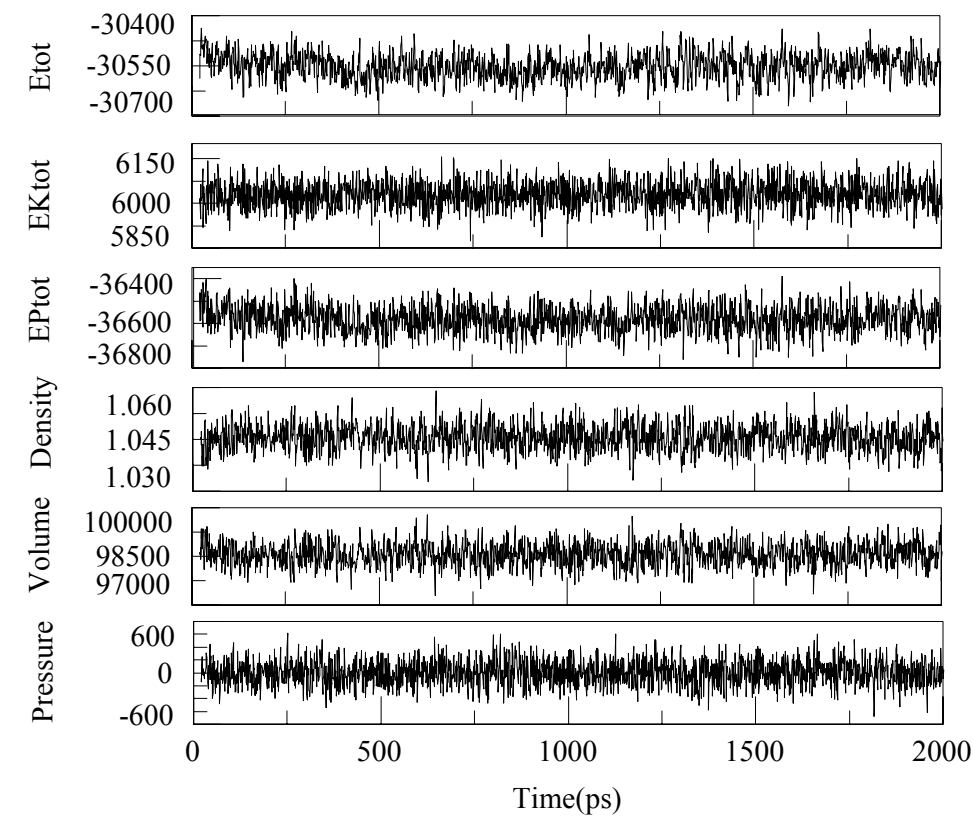

Figure 5.4. Production run for 2 ns of the unmodified B-DNA. Total (Etot), kinetic (EKtot), and potential (EPtot) energies are in $\mathrm{kcal} / \mathrm{mole}$; density is in $\mathrm{g} / \mathrm{cm}^{3}$; pressure is in atmospheres, and volume in $\AA^{3}$.

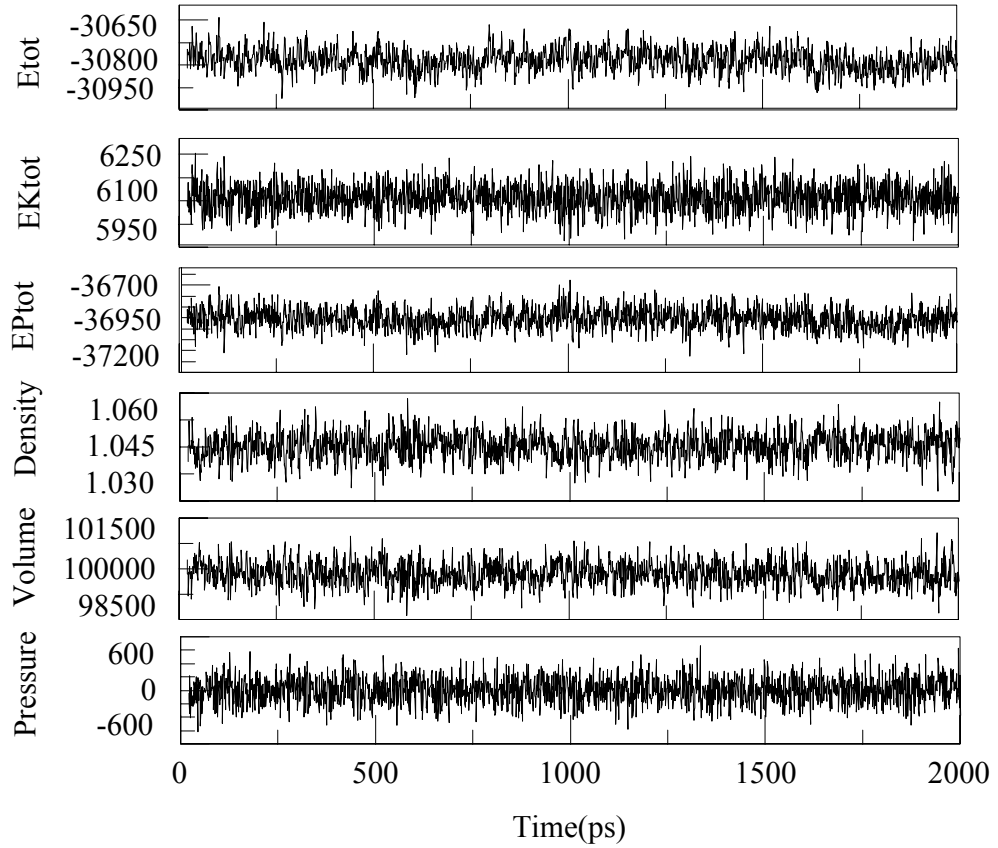

Figure 5.5. Production run for 2 ns of the phenyl-modified B-DNA. Total (Etot), kinetic (EKtot), and potential (EPtot) energies are in $\mathrm{kcal} / \mathrm{mole}$; density is in $\mathrm{g} / \mathrm{cm}^{3}$; pressure is in atmospheres, and volume in $\AA^{3}$. 


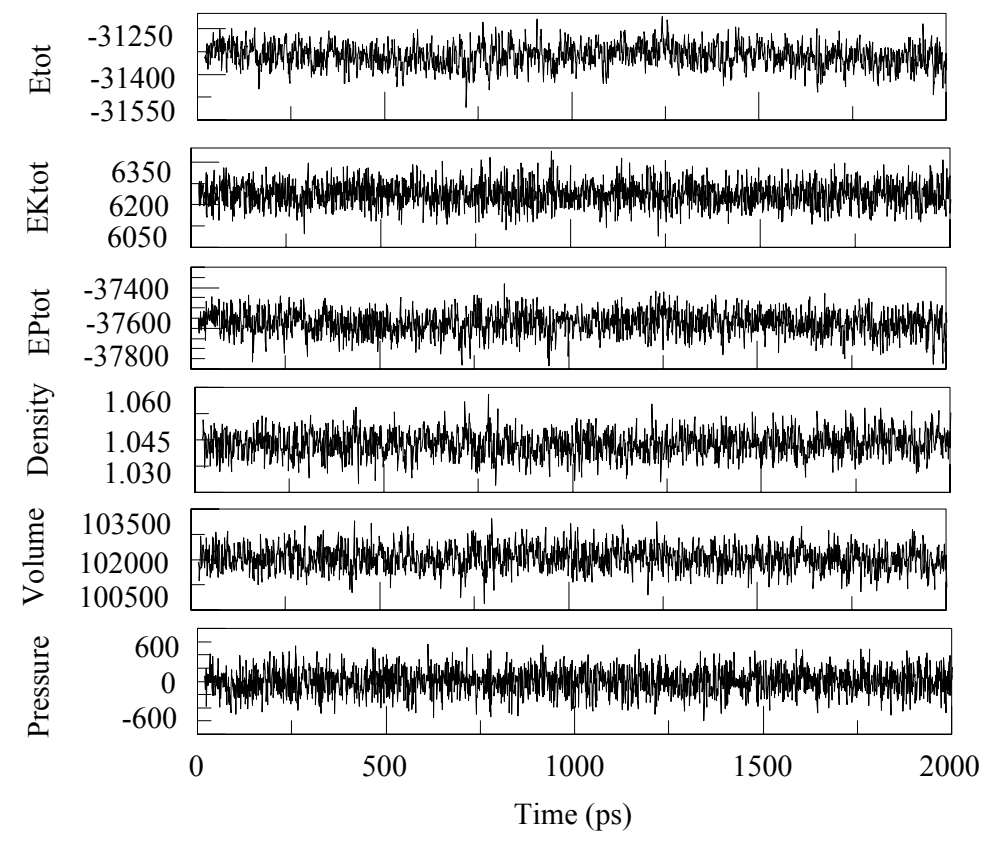

Figure 5.6. Production run for 2 ns of the tolyl-modified B-DNA. Total (Etot), kinetic (EKtot), and potential (EPtot) energies are in $\mathrm{kcal} / \mathrm{mole}$; density is in $\mathrm{g} / \mathrm{cm}^{3}$; pressure is in atmospheres, and volume in $\AA^{3}$.

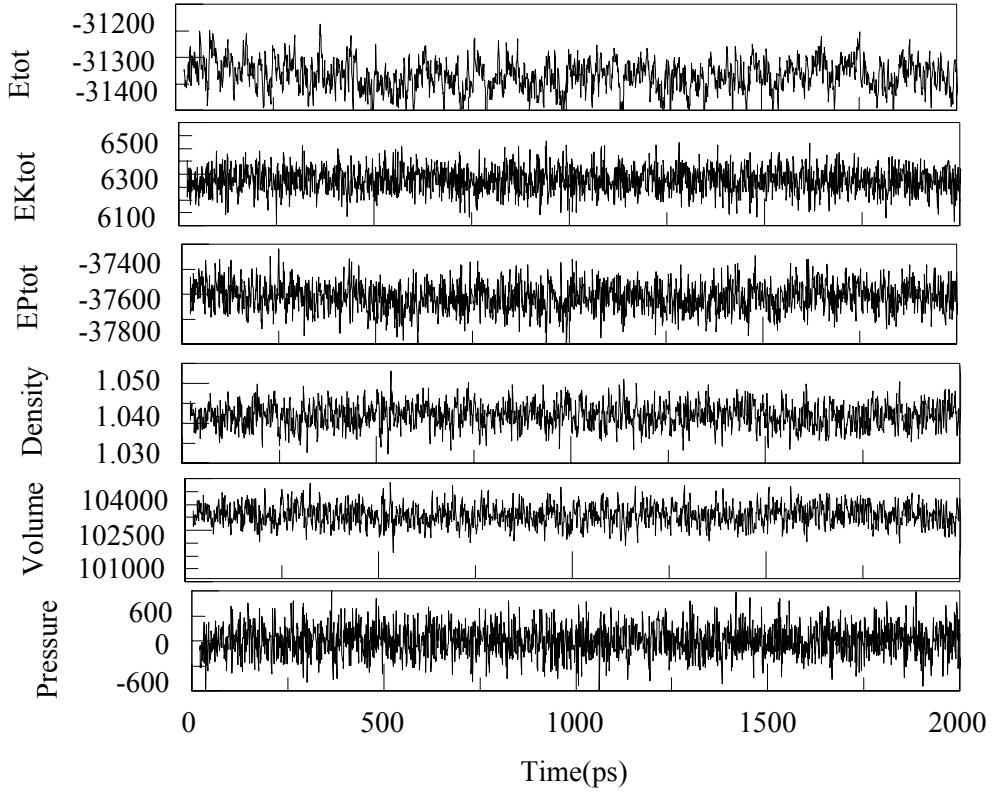

Figure 5.7. Production run for 2 ns of the hydroxymethylphenyl-modified B-DNA. Total (Etot), kinetic (EKtot), and potential (EPtot) energies are in $\mathrm{kcal} / \mathrm{mole}$; density is in $\mathrm{g} / \mathrm{cm}^{3}$; pressure is in atmospheres, and volume in $\AA^{3}$. 


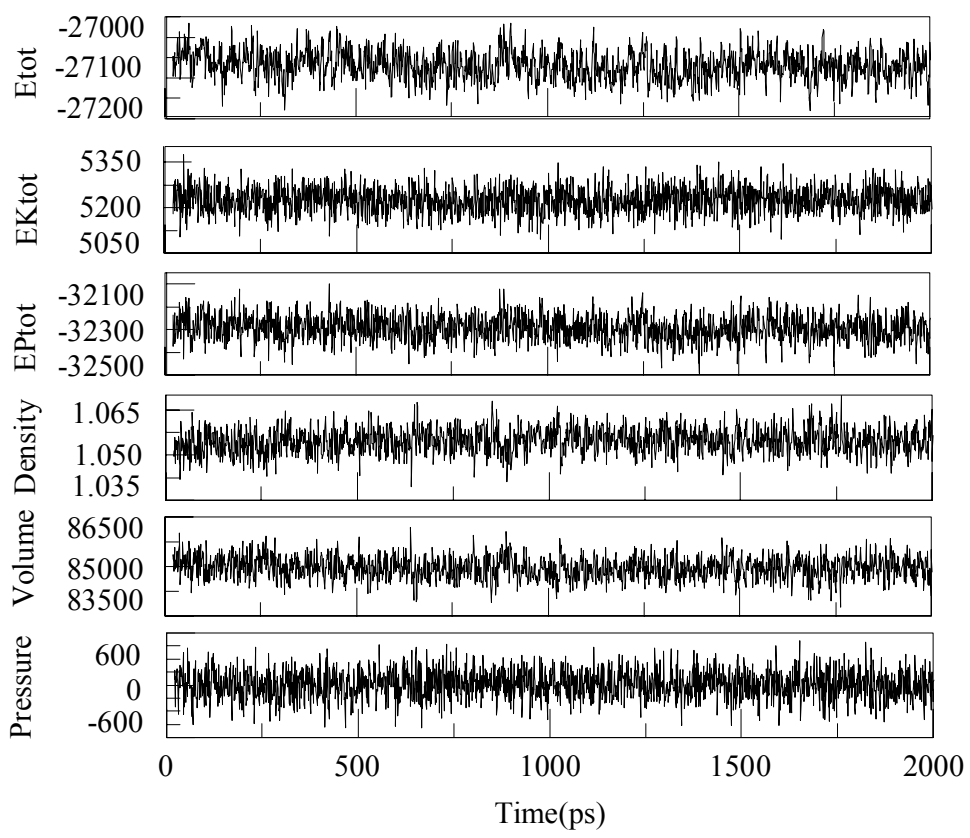

Figure 5.8. Production run for 2 ns of the unmodified Z-DNA. Total (Etot), kinetic (EKtot), and potential (EPtot) energies are in $\mathrm{kcal} / \mathrm{mole}$; density is in $\mathrm{g} / \mathrm{cm}^{3}$; pressure is in atmospheres, and volume in $\AA^{3}$.

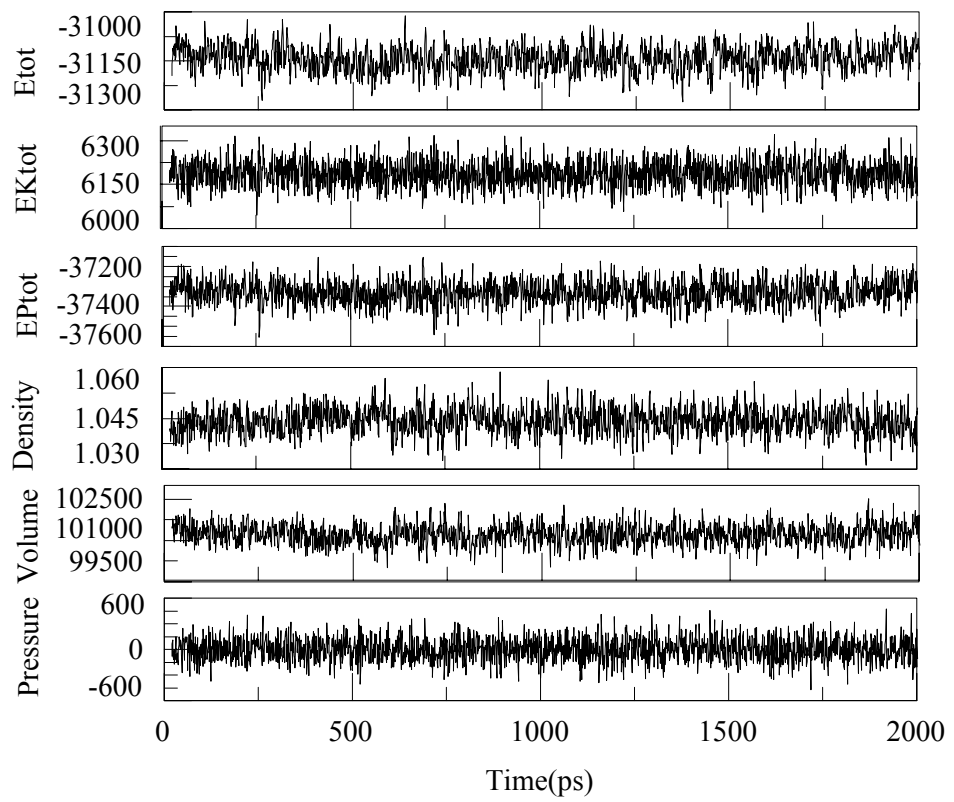

Figure 5.9. Production run for 2 ns of the phenyl-modified Z-DNA. Total (Etot), kinetic (EKtot), and potential (EPtot) energies are in $\mathrm{kcal} / \mathrm{mole}$; density is in $\mathrm{g} / \mathrm{cm} 3$; pressure is in atmospheres, and volume in $\AA^{3}$. 


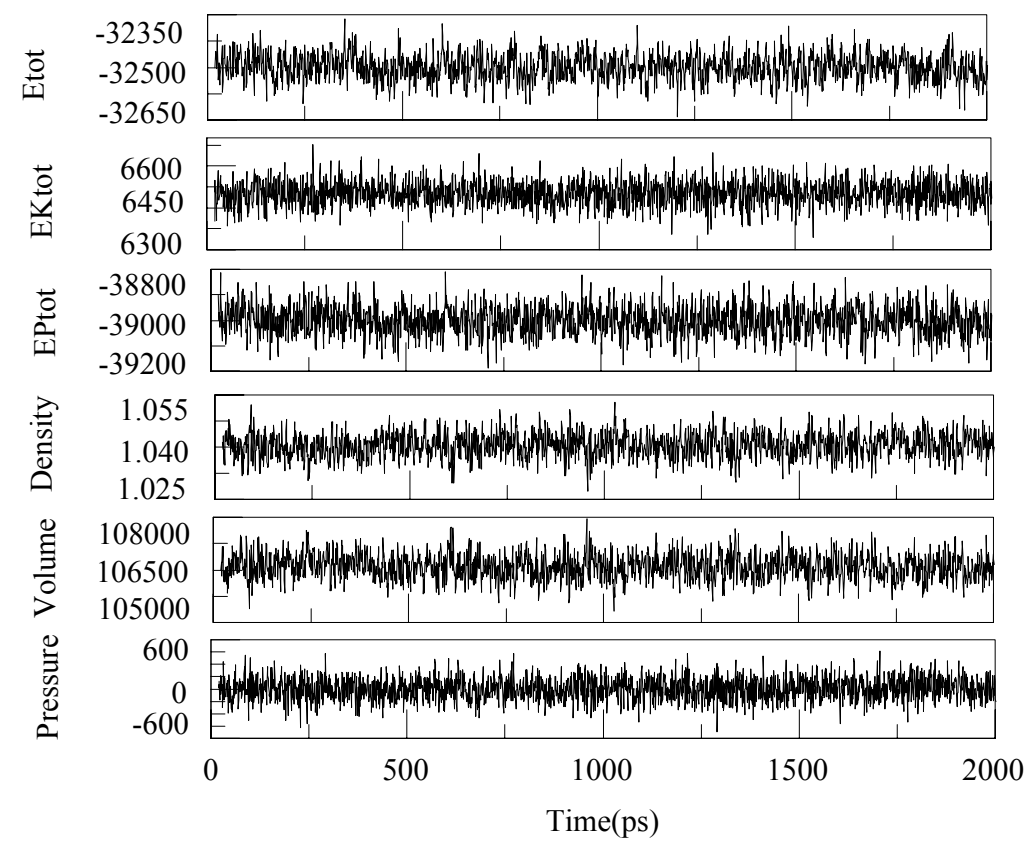

Figure 5.10. Production run for 2 ns of the tolyl-modified Z-DNA. Total (Etot), kinetic (EKtot), and potential (EPtot) energies are in $\mathrm{kcal} / \mathrm{mole}$; density is in $\mathrm{g} / \mathrm{cm}^{3}$; pressure is in atmospheres, and volume in $\AA^{3}$.

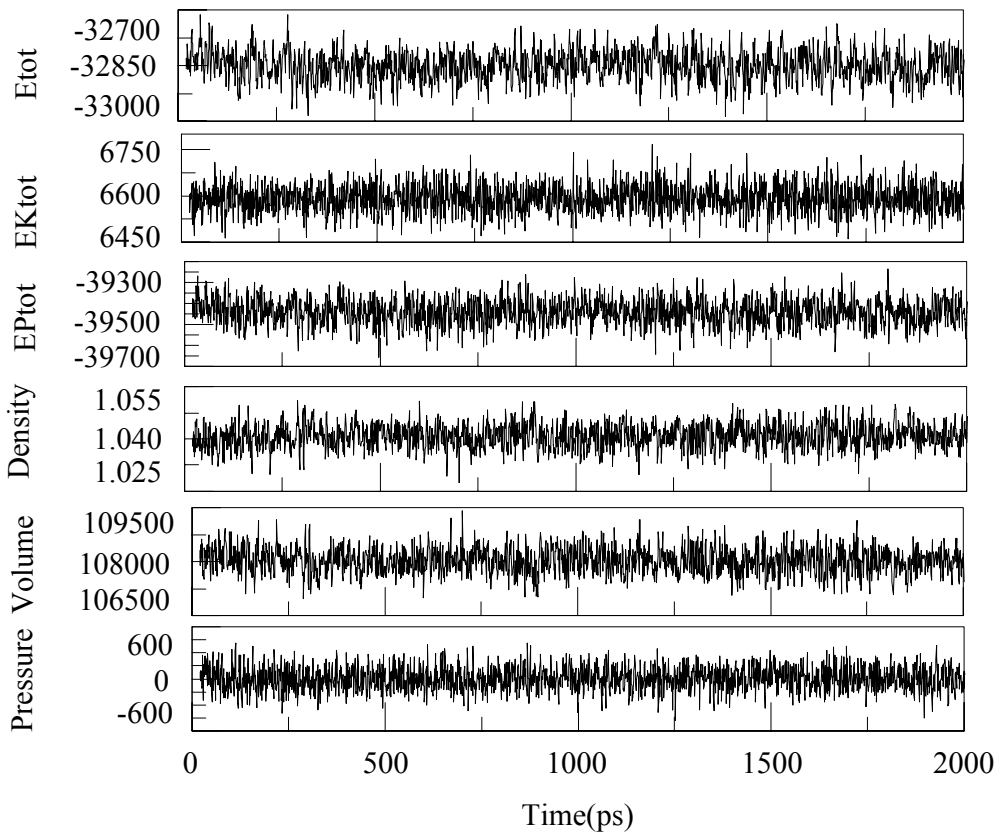

Figure 5.11. Production run for 2 ns of the hydroxymethylphenyl-modified Z-DNA. Total (Etot), kinetic (EKtot), and potential (EPtot) energies are in $\mathrm{kcal} / \mathrm{mole}$; density is in $\mathrm{g} / \mathrm{cm}^{3}$; pressure is in atmospheres, and volume in $\AA^{3}$. 


\subsubsection{Cluster Analysis}

The most representative structure for each conformer was generated using the Cluster trajectory option in the Dynamics Menu of MOIL-view. ${ }^{140}$ The time averaged structure of a dynamics run can be considered representative, but in reality, it may not correspond to a physically sampled structure and may actually be distorted, especially for bonds and angles involving hydrogen atoms. A simple round of minimization may or may not bring the average structure back to reality. On the other hand, the most representative structure is a frame in the trajectory; as determined by a cluster analysis it is the most populated conformation or the conformation that is closest to all others in a simulation.

Trajectories were divided into 250 frame pieces using the ptraj module of Amber, necessary due to size limitations in MOIL-view for this particular trajectory analysis tool. The steps performed in the Cluster analysis program are: (i) each frame is initially placed in a cluster by itself; (ii) a cutoff distance is specified (2 angstroms for our analysis); (iii) based on a 2DRMSD matrix generated, the average RMSD between all pairs of structures for each pair of clusters is calculated; (iv) the average RMSD for the most similar of these pairs is compared to the specified cutoff value, and (v) if the value is less than the cutoff value, the two clusters are combined. This process is repeated until all cluster pairs have an average RMSD greater than the cutoff value. The most representative structure of each cluster is that structure which has the lowest average RMSD to all other members of its cluster. The most representative structures were used in several post-analysis processing techniques and are shown in Figures 5.12 -5.19. 
a)

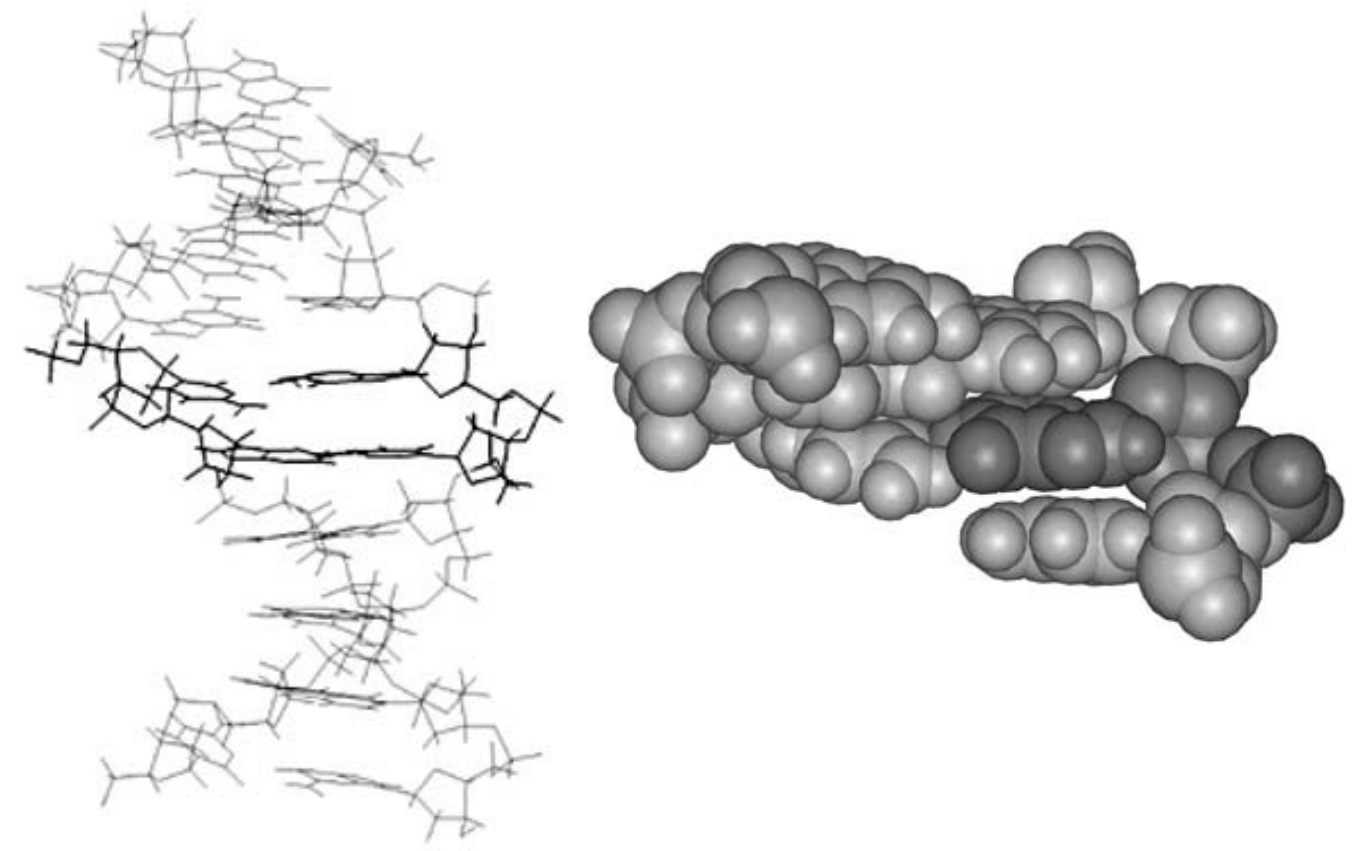

Figure 5.12. Most representative structure from the $\mathbf{C G}$ simulation in the B-DNA form a) side view and b) close up of (C5G6C7) and the complementary strand (G14C15G16). The base shown in bold in this and the following figures corresponds to G6.

a)

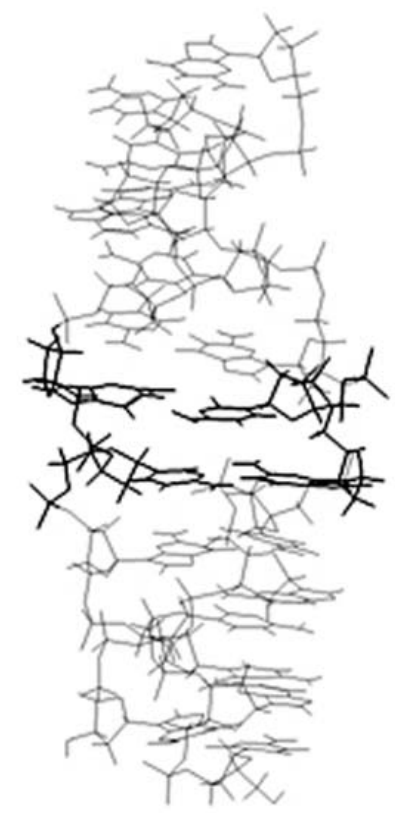

b)

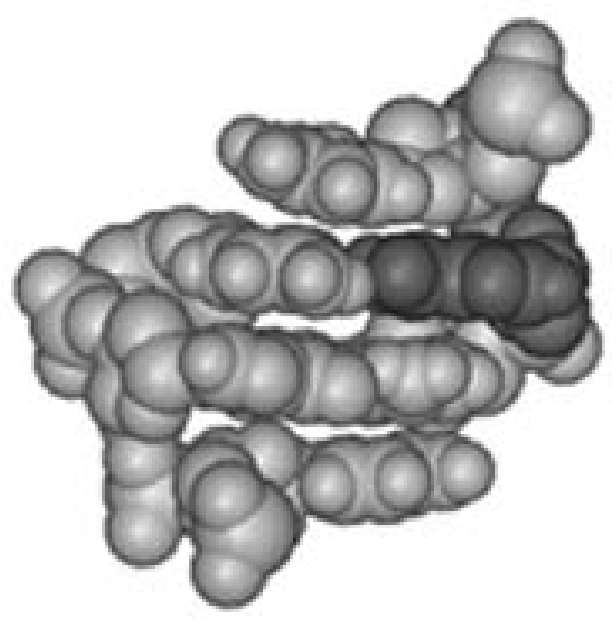

Figure 5.13. Most representative structure from the CG simulation in the Z-DNA form a) side view and b) close up of C5G6C7) and the complementary strand (G14C15G16). 
a)

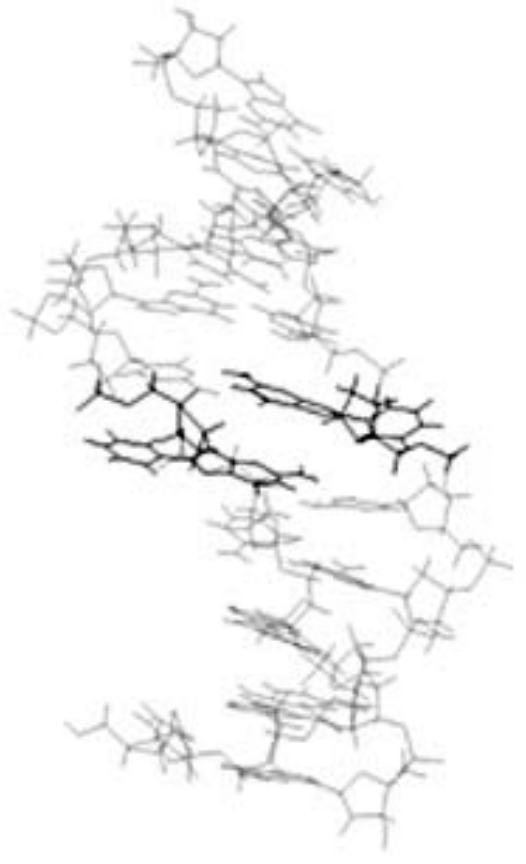

b)

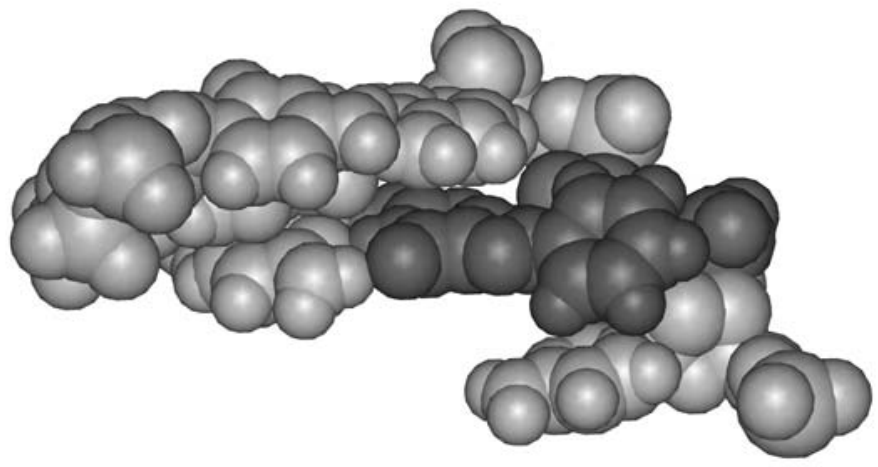

Figure 5.14. Most representative structure from the $\mathbf{C G}^{\mathbf{8 - P h}}$ simulation in the B-DNA form a) side view and b) close up of (C5G6C7) and the complementary strand (G14C15G16).

a)

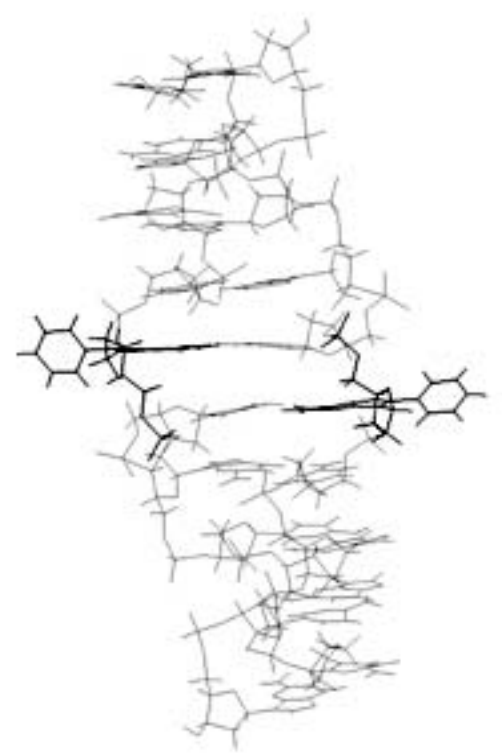

b)

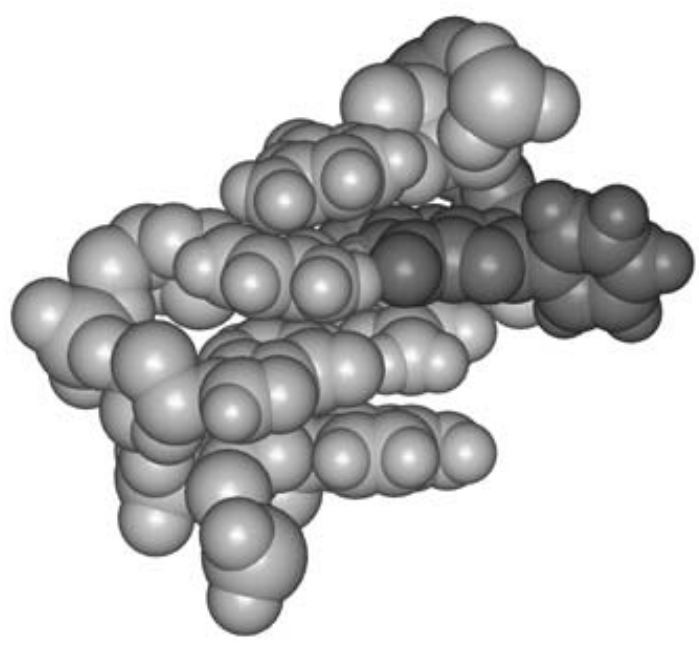

Figure 5.15. Most representative structure from the $\mathbf{C G}^{\mathbf{8 - P h}}$ simulation in the Z-DNA form a) side view and b) close up of (C5G6C7) and the complementary strand (G14C15G16). 
a)

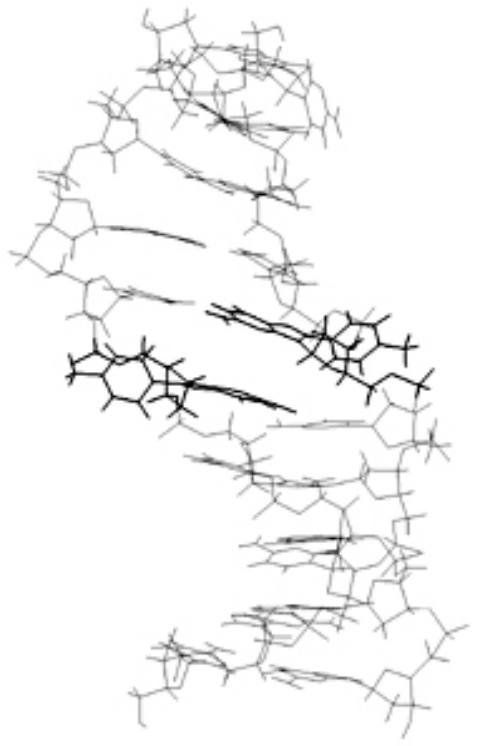

b)

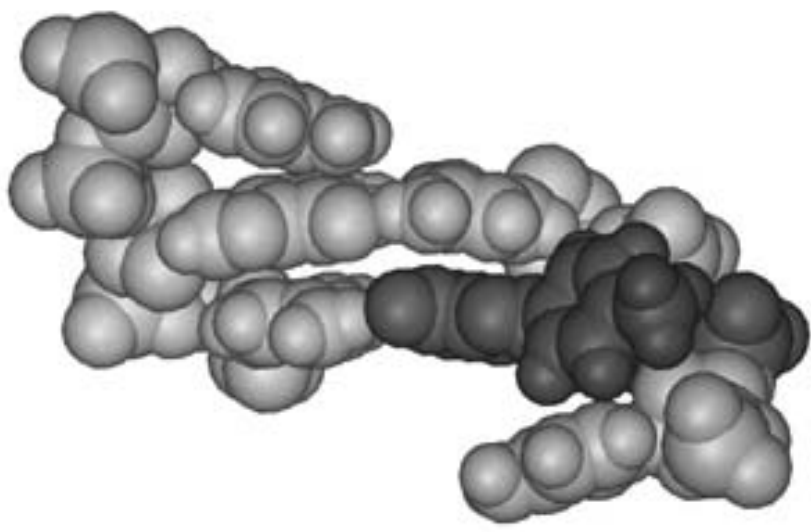

Figure 5.16. Most representative structure from the $\mathbf{C G}^{\mathbf{8 - T o l}}$ simulation in the B-DNA form a) side view and b) close up of (C5G6C7) and the complementary strand (G14C15G16).

a)

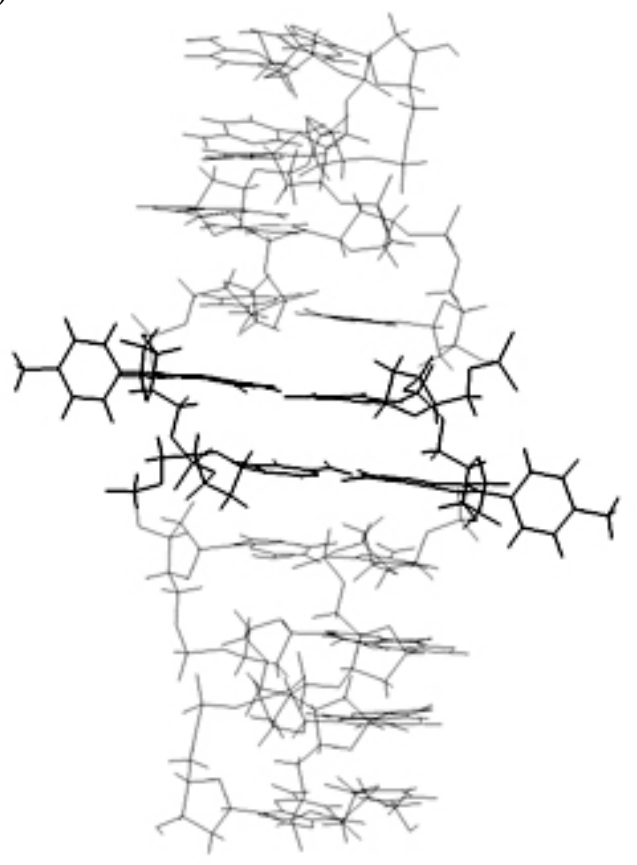

b)

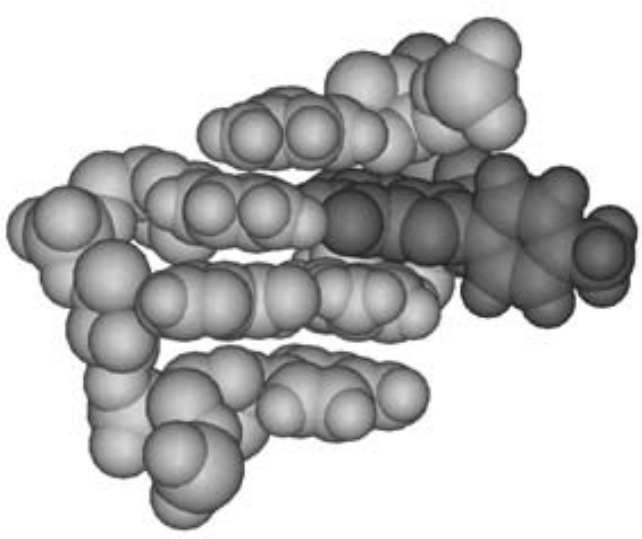

Figure 5.17. Most representative structure from the $\mathbf{C G}^{\mathbf{8}-\mathrm{Tol}}$ simulation in the Z-DNA form a) side view and b) close up of (C5G6C7) and the complementary strand (G14C15G16). 
a)

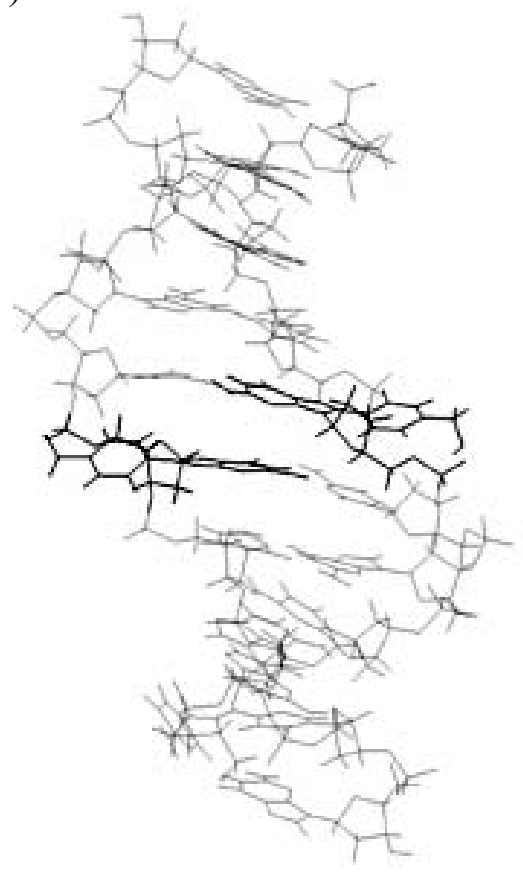

b)

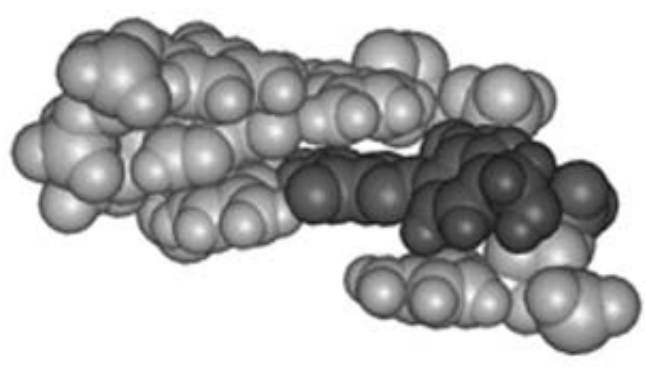

Figure 5.18. Most representative structure from the $\mathbf{C G}^{\mathbf{8 - H m p}}$ simulation in the B-DNA form a) side view and b) close up of (C5G6C7) and the complementary strand (G14C15G16).

a)

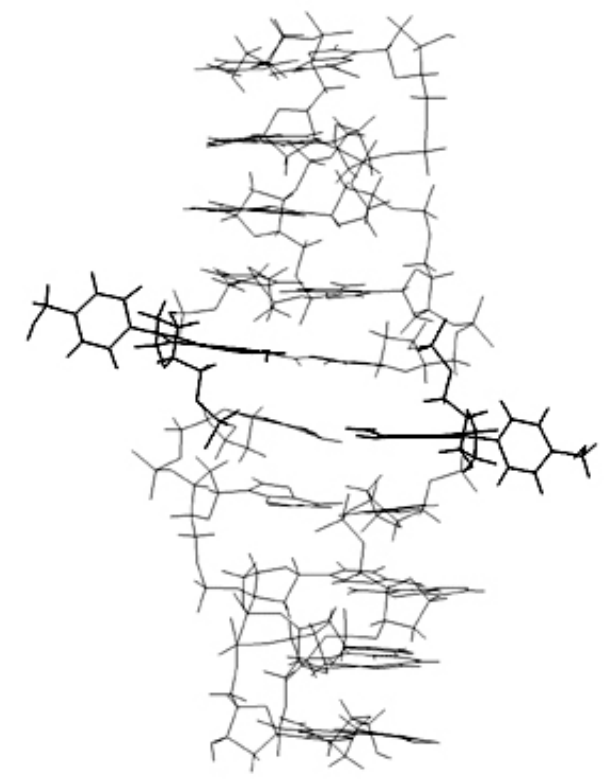

b)

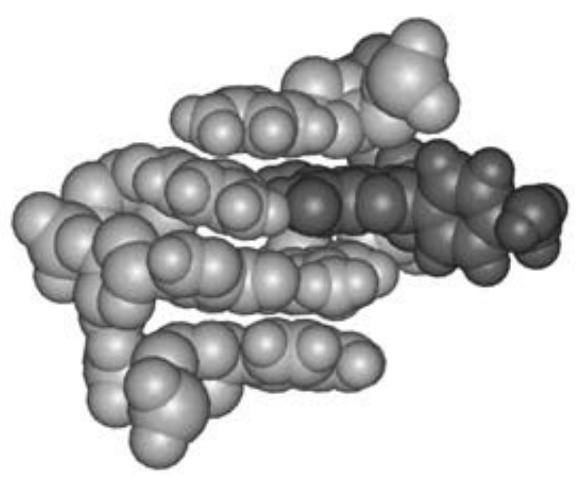

Figure 5.19. Most representative structure from the $\mathbf{C G}^{\mathbf{8}-\mathrm{Hmp}}$ simulation in the Z-DNA form a) side view and b) close up of (C5G6C7) and the complementary strand (G14C15G16). 
The models, in particular the most representative structures, provide the means to determine the average distances between protons on the phenyl ring and those on C5, G6, and C7. The shorter distances (less than $4 \AA$ ) were determined from the most representative structure for the B-DNA between the phenyl protons and the rest of the oligonucleotide, then MOIL-view was used to determine the average distance over the course of the dynamics run. These average distances are phenyl-H10/G6-H2' (2.33 Å), phenyl-H10/G6-H3' (2.98 Å), phenyl-H12/C5-H2'

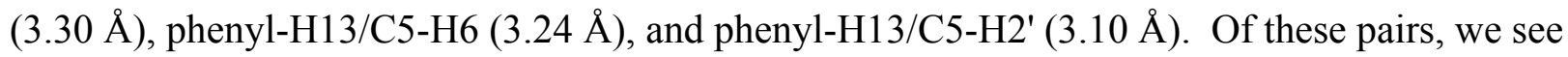
NOESY correlations from phenyl-H10/G6-H2' and phenyl-H10/G6-H3', i.e., those with the shortest calculated differences. Unfortunately, the only NOESY correlations that we clearly observe were to the modified bases' own sugar protons. We did not observe any crosspeaks between the phenyl protons and any of the sugar protons of $\mathrm{C} 5$, which would help to firmly establish the conformation of the glycosidic bond of G6. However, the anti conformation seems most likely, since in the syn conformation, the base pairing in the $\mathrm{C}: \mathrm{G}^{8-\mathrm{Ph}}$ base pair would be lost, thus destabilizing the duplex. In addition, this conformation is consistent with the large upfield shifts of the phenyl-H10 and H11 protons. In the Z-DNA form, the phenyl group is completely outside of the helix, and it can freely rotate, rendering the ortho and the meta protons equivalent. Calculations of the time averaged distances between the phenyl and neighboring base and backbone protons for the modified Z-DNA found only one distance close to $3 \AA$, Ph-H10/ C5-

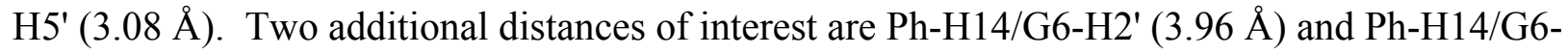
H1' (3.58 $\AA$ ). Of these pairs only the Ph-H10/C5-H5' and Ph-H14/G6-H1' NOESY correlations were observable. 


\subsubsection{Trajectory-Root-Mean-Squared-Deviations}

The root-mean-square deviations (RMSD) on all atoms over the course of the MD trajectories from (i) the most representative and (ii) the starting structures of the DNA's are shown in Figures 5.20 and 5.21, respectively. The RMSD calculations were performed using MOIL-view but the ptraj or carnal modules in Amber can be used as well. By comparing B to ZDNA for $\mathbf{C G}, \mathbf{C G}^{\mathbf{8 - P h}}, \mathbf{C G}^{\mathbf{8 - T o l}}$, and $\mathbf{C G}^{\mathbf{8 - H m p}}$, one can see greater fluctuations in the B-DNA's relative to the Z-DNA's. This is to be expected due to the relative greater rigidity of Z-DNA. This phenomenon can also be observed in the vibrational entropy calculations from the MMPBSA module of Amber described in section 5.4.5.

Several areas in Figure 5.20a have relatively large RMSD's to the most representative structures. This is especially conspicuous in the first few hundred ps of the $\mathbf{C G}^{\mathbf{8}-\mathrm{Tol}}$ simulation. The cluster analysis was performed by dividing the trajectory into eight sections; a cutoff of $2 \AA$ was used to find the most representative structure for each section and then these eight structures were used to determine the most representative structure for the simulation. It is, therefore, possible for the most representative structure of the trajectory to have an RMSD of up to 4 angstroms from any one frame. For the majority of the frames in each trajectory, the RMSD is within 1-2 angstroms of the most representative structure. The spikes down to zero denote the frame in the trajectory that is the most representative structure.

Figures 5.22a and 5.22b show the RMSD of the trajectories for all of the B-DNA structures to canonical A and B-DNA, respectively. All four trajectories deviate from both canonical structures; although the unmodified structure goes away from the canonical B-DNA towards A-DNA relative to the start as the simulation progresses. Figure 5.22c compares the trajectories of the modified B-DNA's to the most representative structure from the unmodified 
B-DNA trajectory. The comparison of the modified DNA's to the unmodified is substituent dependent with $\mathbf{C G}^{\mathbf{8 - H m p}}$ staying closest to the unmodified structure followed by $\mathbf{C G}^{\mathbf{8 - P h}}$ and $\mathbf{C G}^{8 \text {-Tol }}$.

Figure 5.23a shows the RMSD of the trajectories for all of the Z-DNA structures to canonical Z-DNA, and Figure 5.23b compares the trajectories of the modified Z-DNA's to the most representative structure from the unmodified Z-DNA trajectory. The drift for the modified Z's from canonical and from the unmodified Z-DNA is less than that observed with the BDNA's owing to the rigidity of Z-DNA in general. It can be observed from both Figure 5.23a and Figure 5.23b that the tolyl-modified Z-DNA drifts farther from canonical and unmodified then do the phenyl and hydroxymethyl-modified oligos. 
a)
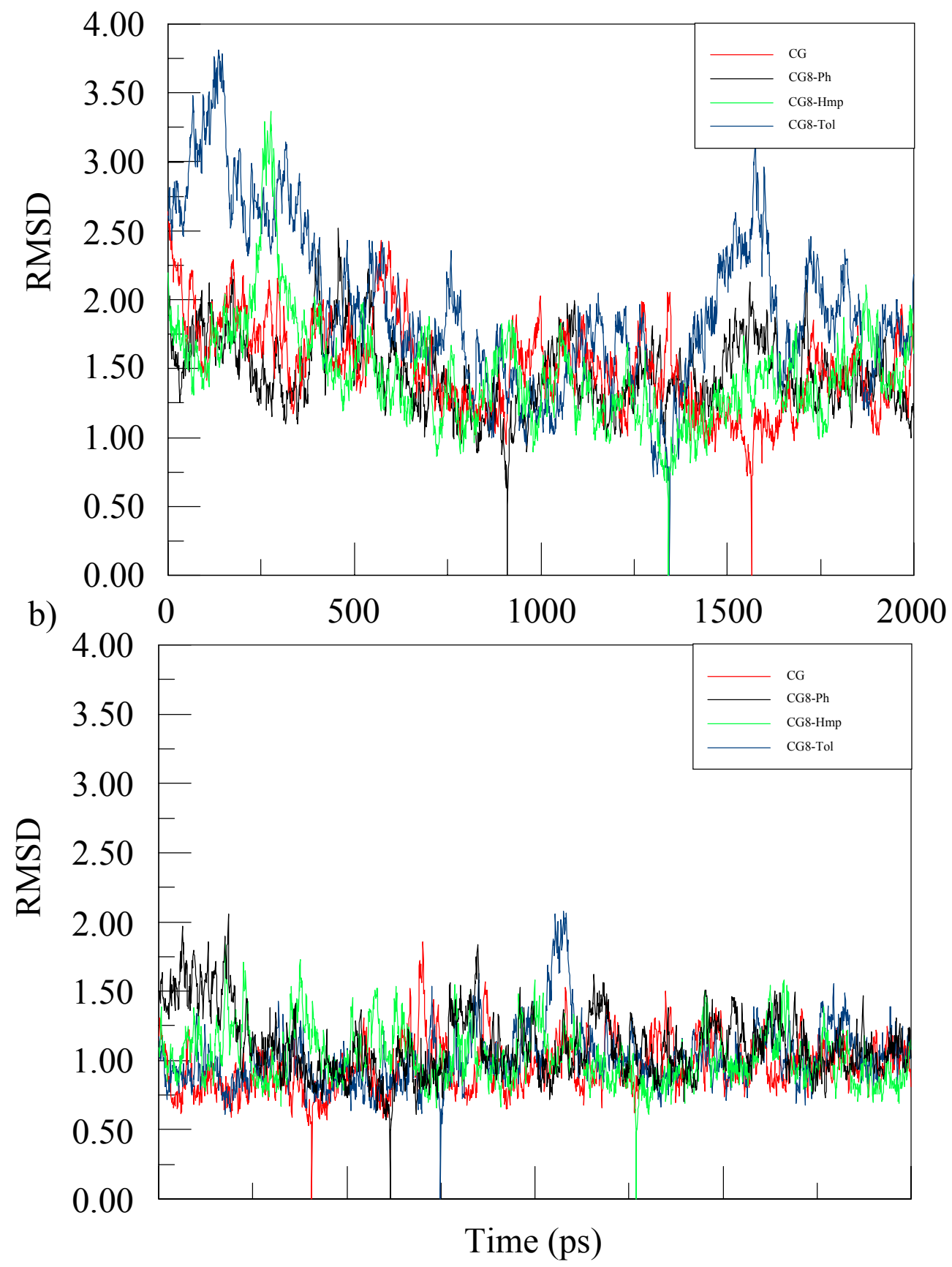

Figure 5.20. RMSD's to the most representative structures over the course of the trajectories of a) the B-DNA's and b) the Z-DNA's. 
a)

b)
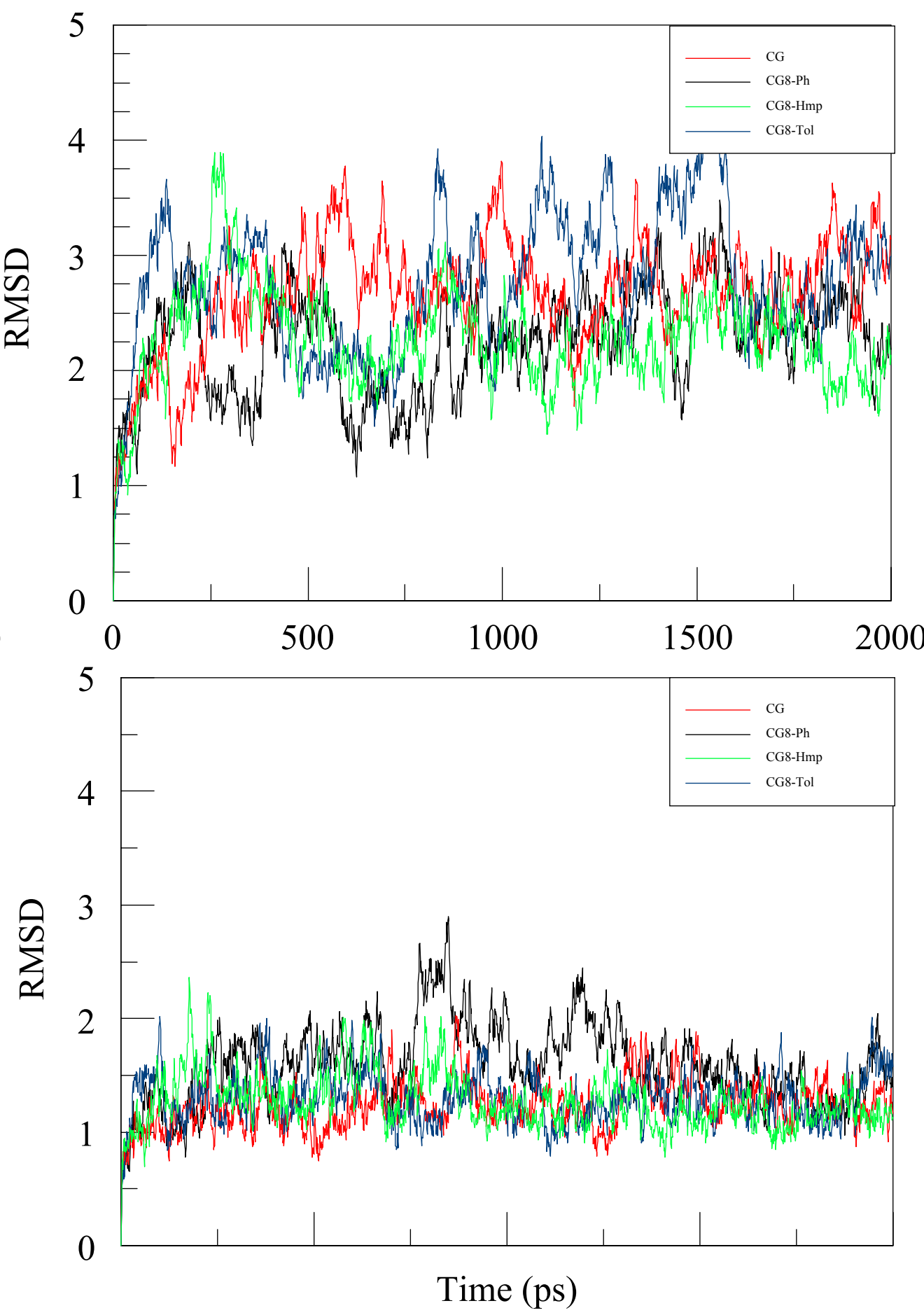

Figure 5.21. RMSD's to the starting structures over the course of the trajectories of a) the BDNA's and b) the Z-DNA's. 
a)

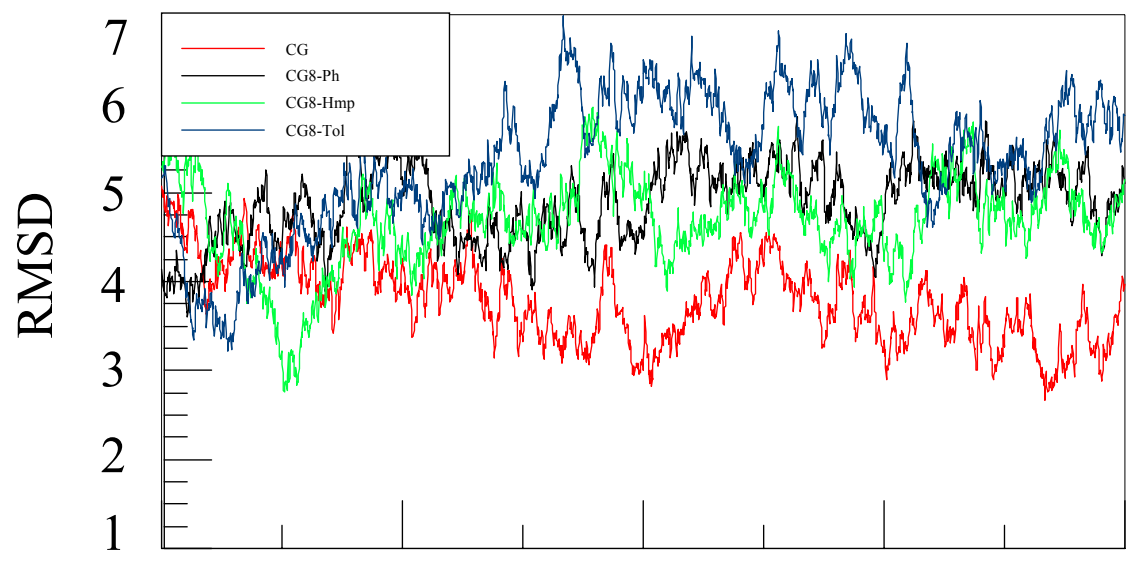

b)

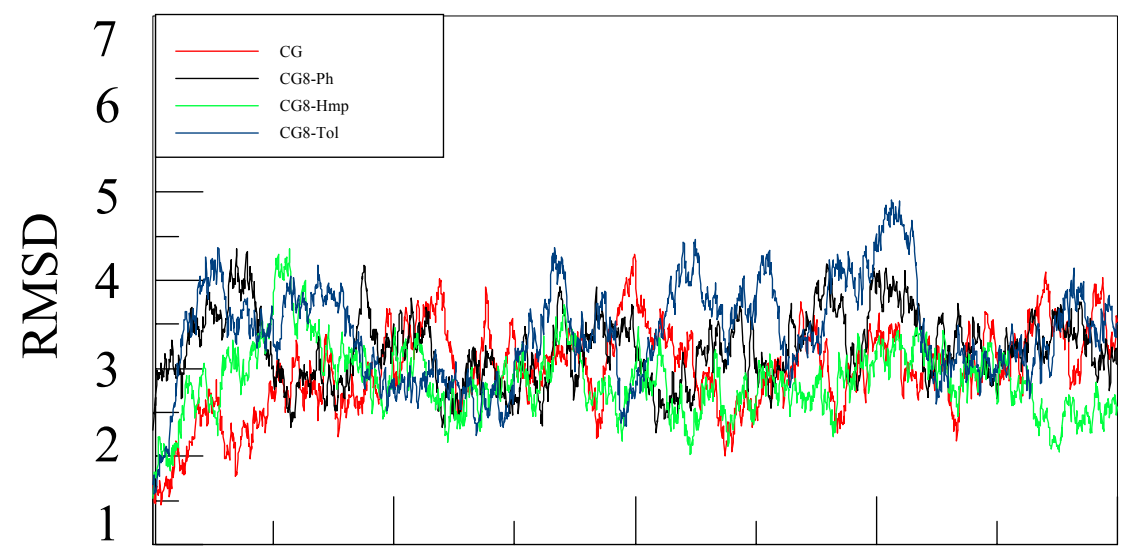

c)

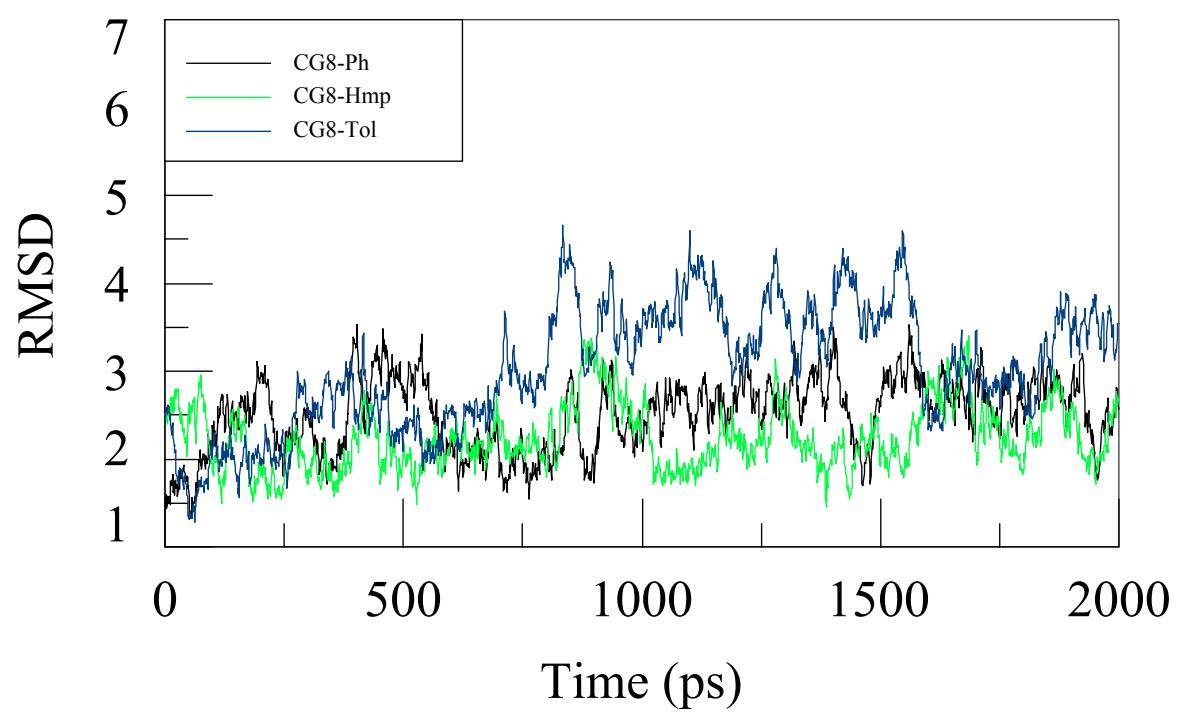

Figure 5.22. a) RMSD's of $\mathbf{C G}, \mathbf{C G}^{8-\mathrm{Ph}}, \mathbf{C G}^{8-\mathrm{Tol}}$, and $\mathbf{C G}^{\text {-Hmp }}$ in the B-DNA conformation to canonical A-DNA, b) RMSD's of $\mathbf{C G}, \mathbf{C G}^{8-\mathrm{Ph}}, \mathbf{C G}^{8-\mathrm{Tol}}$, and $\mathbf{C G}^{8-\mathrm{Hmp}}$ in the B-DNA conformation to canonical B-DNA, and c) RMSDs of $\mathbf{C G}^{8-\mathrm{Ph},} \mathbf{C G}^{8-\mathrm{Tol}}$, and $\mathbf{C G}^{\mathbf{8 - H m p}}$ in the BDNA conformation to the most representative structure of the unmodified B-DNA. 
a)

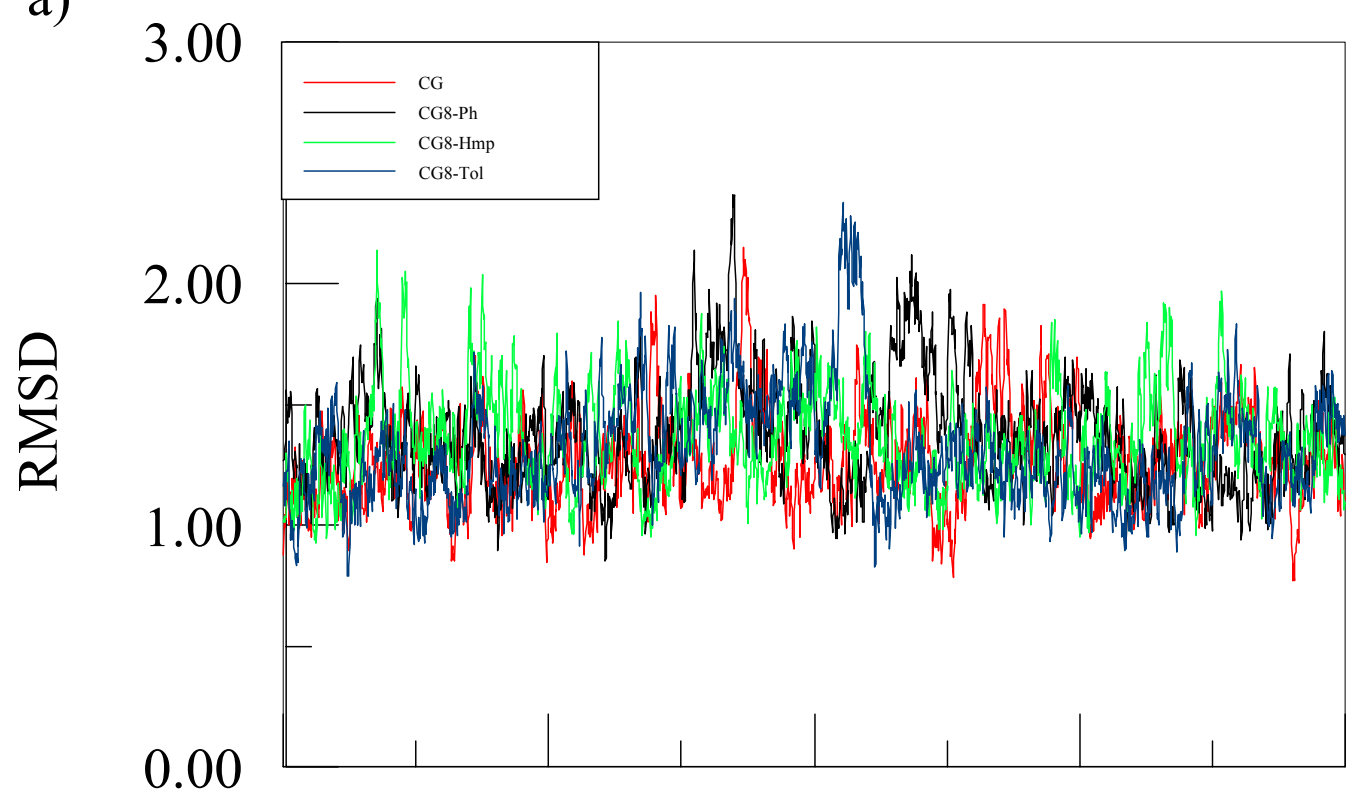

b)

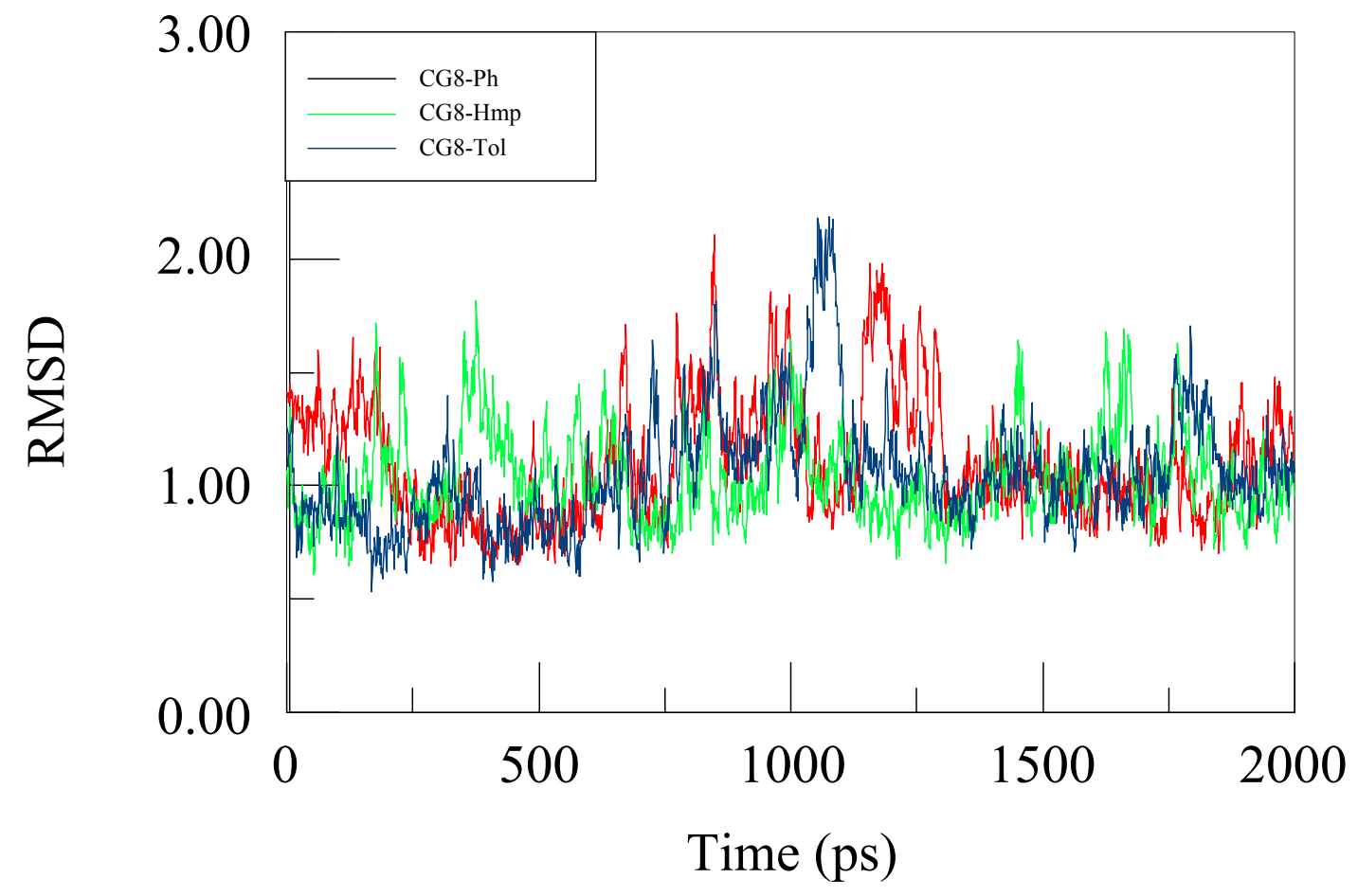

Figure 5.23. a) RMSD's of $\mathbf{C G}, \mathbf{C G}^{8-\mathrm{Ph}}, \mathbf{C G}^{\mathbf{8 - T o l}}$, and $\mathbf{C G}^{\text {--Hmp }}$ in the Z-DNA conformation to canonical Z-DNA, and b) RMSD's of $\mathbf{C G}, \mathbf{C G}^{8-\mathrm{Ph}}, \mathbf{C G}^{8-\mathrm{Tol}}$, and $\mathbf{C G}^{\text {-Hmp }}$ in the Z-DNA conformation to the most representative structure of the unmodified Z-DNA. 


\subsubsection{Structural Analysis}

As mentioned earlier, the most representative structure has several useful applications. Using the program CURVES, ${ }^{137,138}$ it is possible to extract structural information in the form of torsional angles, groove parameters, and various movements involving two bases of a pair or two sets of base pairs in a base step. When using a reasonable representative structure, these values are helpful in comparing conformational features of different segments within a DNA or in comparing one DNA to another. The average values of torsional angles from crystal structures are given in Table $5.7^{141}$ with the values for canonical DNA helices as calculated by CURVES shown in parentheses. The torsion angles are defined in Figure 5.24a.

Table 5.7. Average torsion angles (in degrees) from crystal structures ${ }^{141}$ and as calculated by CURVES for canonical structures in parentheses.

\begin{tabular}{|l|l|l|l|l|l|l|l|}
\hline Type & $\alpha$ & $\beta$ & $\gamma$ & $\delta$ & $\varepsilon$ & $\chi$ & $\zeta$ \\
\hline \hline A-DNA $^{\mathrm{a}}$ & & & & & & & \\
& -50 & 172 & 41 & 79 & -146 & -154 & -75 \\
& $(-84)$ & $(-152)$ & $(45)$ & $(84)$ & $(179)$ & $(-154)$ & $(-49)$ \\
\hline B-DNA C residues $^{\mathrm{a}}$ & -30 & 126 & 47 & 143 & 85 & -97 & -169 \\
& $(-47)$ & $(-146)$ & $(36)$ & $(156)$ & $(155)$ & $(-95)$ & $(-98)$ \\
\hline B-DNA G residues $^{\mathrm{a}}$ & -66 & 145 & 22 & 147 & -156 & -74 & -158 \\
& $(-47)$ & $(-146)$ & $(36)$ & $(156)$ & $(155)$ & $(-95)$ & $(-98)$ \\
\hline Z-DNA C residues $^{\mathrm{b}}$ & -140 & -137 & 51 & 138 & -97 & 82 & -154 \\
& $(-136)$ & $(-139)$ & $(55)$ & $(138)$ & $(-94)$ & $(81)$ & $(-159)$ \\
\hline Z-DNA G residues $^{\mathrm{b}}$ & 52 & 179 & -174 & 95 & -104 & -65 & 59 \\
& $(47)$ & $(178)$ & $(-169)$ & $(100)$ & $(-104)$ & $(-69)$ & $(67)$ \\
\hline
\end{tabular}

${ }^{\mathrm{a}}$ Calculated from A and B-DNA structures generated by the nucgen module of Amber. ${ }^{\mathrm{b}}$ Calculated from a Z-DNA structure generated by Sybyl.

Table 5.7 shows that there are some discrepancies between those values reported in the literature and the values calculated by CURVES. For example, there is a $34^{\circ}$ difference in the values of alpha for A-DNA. These differences are predominately a product of the method used to perform the calculations. For consistency's sake, we report all values as calculated by CURVES, and most of our comparisons are from one structure to another within this work. 
The groove parameters shown in Tables 5.8, 5.9, and 5.10 have been defined and measured with respect to the helical axis system, ${ }^{138}$ defined in Figure 5.24b below. The x-axis points in the direction of the major groove along the pseudo-dyad axis of an ideal Watson-Crick base pair, i.e., the perpendicular bisector of the $\mathrm{C1}$ '-C1' vector spanning the base pair. ${ }^{142}$ The $y$ axis runs along the long axis of the idealized base pair parallel to the $\mathrm{C} 1$ '- $\mathrm{C} 1$ ' vector. The z-axis (not shown) is defined by the right hand rule, consequently for right-handed A and B-DNA the zaxis points along the $5^{\prime}$ to $3^{\prime}$ direction of the strand.

a)

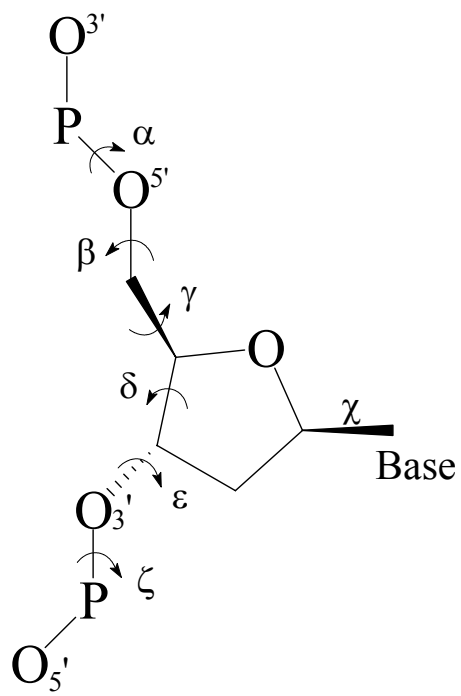

b)

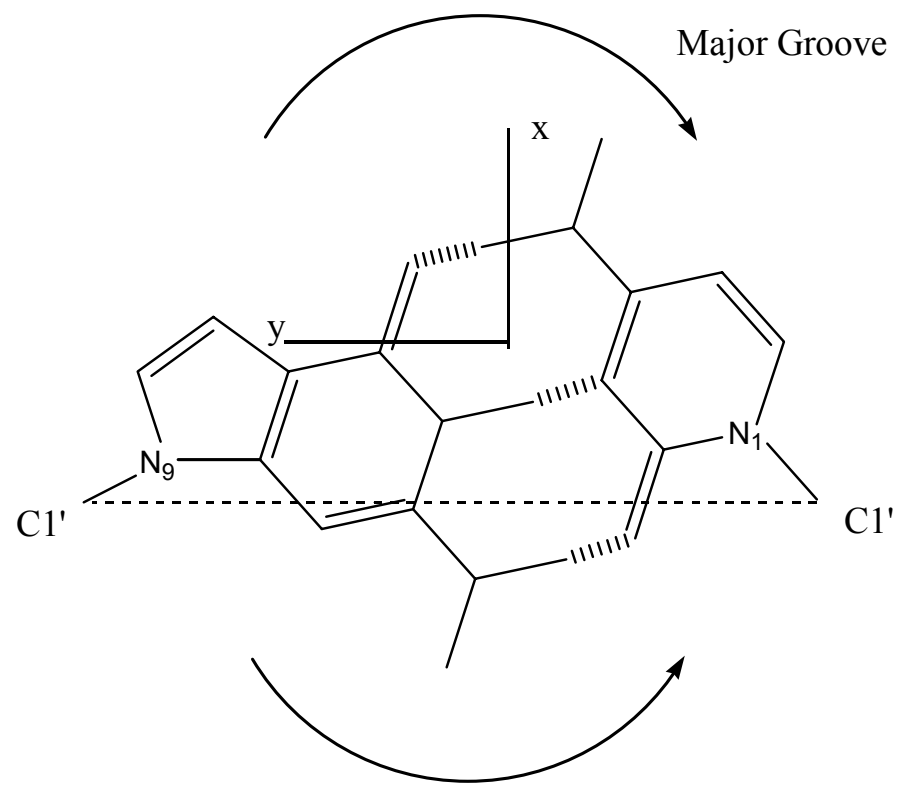

Minor Groove

Figure 5.24. Definition of a) torsional angles and b) the helical axis system. ${ }^{143}$ All arrows point in the positive direction. 
Tables 5.8, 5.9, and 5.10 outline the groove and axis parameters for the modified and unmodified B and Z-DNA. In the B-DNA conformation, the modifications cause the major groove to become wider and shallower while the minor groove becomes narrower relative to the unmodified. These differences are most notable for the tolyl-modified DNA. The modifications do not have a significant effect on the groove and axis parameters for the modified DNA's in the $\mathrm{Z}$ conformation. 
Table 5.8a. Global groove width and depth parameters for unmodified and modified duplexes in the B-DNA conformation.

\begin{tabular}{|c|c|c|c|c|c|}
\hline \multicolumn{6}{|c|}{ Minor Groove Width $(\AA)$} \\
\hline Base \# & CG & $\mathrm{CG}^{8-\mathrm{Ph}}$ & $\mathrm{CG}^{8-\mathrm{Tol}}$ & $\mathrm{CG}^{8-\mathrm{Hmp}}$ & $\mathbf{C G}^{\mathrm{a}}$ \\
\hline 2 & 7.31 & $--^{b}$ & --- & --- & --- \\
\hline 3 & 8.03 & 8.83 & 6.40 & 7.74 & 5.9 \\
\hline 4 & 7.91 & 7.22 & 5.00 & 6.83 & 5.9 \\
\hline 5 & 8.40 & 4.32 & 4.81 & 6.38 & 5.9 \\
\hline 6 & 7.00 & 5.99 & 5.41 & 7.74 & 5.9 \\
\hline 7 & 7.77 & 7.58 & 8.80 & 7.13 & 5.9 \\
\hline \multicolumn{6}{|c|}{ Major Groove Width $(\AA)$} \\
\hline Base \# & CG & $\mathrm{CG}^{8-\mathrm{Ph}}$ & $\mathrm{CG}^{8 \mathrm{Tol}}$ & $\mathrm{CG}^{8-\mathrm{Hmp}}$ & CG* \\
\hline 2 & --- & ---- & --- & 13.07 & --- \\
\hline 3 & 14.56 & --- & 18.92 & 16.44 & 11.1 \\
\hline 4 & 12.20 & 16.80 & 21.94 & 15.96 & 11.1 \\
\hline 5 & 11.85 & $\mathbf{1 7 . 5 0}$ & 23.72 & 16.67 & 11.1 \\
\hline 6 & 11.36 & $\mathbf{1 7 . 5 5}$ & 23.90 & 15.45 & 11.1 \\
\hline 7 & 15.38 & 15.72 & 15.87 & 13.20 & 11.1 \\
\hline \multicolumn{6}{|c|}{ Minor Groove Depth $(\AA)$} \\
\hline Base \# & CG & $\mathrm{CG}^{8-\mathrm{Ph}}$ & $\mathrm{CG}^{8-\mathrm{Tol}}$ & $\mathrm{CG}^{8-\mathrm{Hmp}}$ & CG* \\
\hline 2 & 3.88 & --- & --- & --- & --- \\
\hline 3 & 5.10 & --- & 5.41 & 5.03 & 4.6 \\
\hline 4 & 4.04 & 3.45 & 5.75 & 3.58 & 4.6 \\
\hline 5 & 3.71 & 4.49 & 4.38 & 4.01 & 4.6 \\
\hline 6 & 4.31 & 3.73 & 3.81 & 3.72 & 4.6 \\
\hline 7 & 3.51 & 4.48 & 3.99 & 5.11 & 4.6 \\
\hline \multicolumn{6}{|c|}{ Major Groove Depth $(\AA)$} \\
\hline Base \# & CG & $\mathrm{CG}^{8-\mathrm{Ph}}$ & $\mathrm{CG}^{8 \text {-Tolh }}$ & $\mathrm{CG}^{8-\mathrm{Hmp}}$ & CG* \\
\hline 2 & --- & --- & --- & 0.36 & --- \\
\hline 3 & 2.26 & --- & 2.94 & 6.11 & 4.1 \\
\hline 4 & 7.13 & 6.78 & 3.72 & 6.24 & 4.1 \\
\hline 5 & 9.10 & 4.02 & 4.46 & 5.93 & 4.1 \\
\hline 6 & 6.96 & 6.80 & 4.77 & 6.99 & 4.1 \\
\hline 7 & 6.66 & 1.51 & 5.00 & 5.6 .3 & 4.1 \\
\hline
\end{tabular}

${ }^{\mathrm{a} C}$ Canonical B-DNA as calculated by CURVES. ${ }^{\mathrm{b}}$ CURVES does not yield meaningful values of the groove parameters for all structures. Problems arise from numerical instabilities, as well as from geometrical distortions of the double helix, and from missing backbone atoms. For some structures the groove parameters are not calculated at all. In other cases unusual high and steep peaks occur in the plots. Spikes of the groove width are meaningless. The real width of the grooves changes only smoothly because the radius of the atoms involved is $1.5 \AA$ to $2.0 \AA .{ }^{144}$ 
Table 5.8b. Global groove width and depth parameters for unmodified and modified duplexes in the Z-DNA conformation.

\begin{tabular}{|c|c|c|c|c|c|}
\hline \multicolumn{6}{|c|}{ Minor Groove Width $(\AA)$} \\
\hline Base \# & CG & $\mathrm{CG}^{8-\mathrm{Ph}}$ & $\mathrm{CG}^{8-\mathrm{Tol}}$ & $\mathrm{CG}^{8-\mathrm{Hmp}}$ & $\mathbf{C G}^{\mathbf{a}}$ \\
\hline 2 & 2.65 & 2.51 & 1.96 & 3.28 & 3.2 \\
\hline 3 & 3.57 & 4.47 & 3.76 & 5.24 & 4.2 \\
\hline 4 & 1.93 & 1.49 & 1.91 & 2.23 & 3.0 \\
\hline 5 & 4.15 & 4.79 & 4.79 & 4.29 & 4.2 \\
\hline 6 & 4.13 & 3.75 & 4.22 & 2.63 & 3.0 \\
\hline 7 & 4.13 & 4.98 & 4.13 & 4.27 & 4.2 \\
\hline \multicolumn{6}{|c|}{ Major Groove Width (§) } \\
\hline Base \# & $\mathbf{C G}$ & $\mathrm{CG}^{8-\mathrm{Ph}}$ & $\mathrm{CG}^{8-\mathrm{Tol}}$ & $\mathrm{CG}^{8-\mathrm{Hmp}}$ & $\mathrm{CG}^{*}$ \\
\hline 2 & --- & --- & --- & --- & --- \\
\hline 3 & 11.32 & --- & --- & 11.16 & --- \\
\hline 4 & 12.28 & 10.92 & 13.27 & 12.92 & 11.0 \\
\hline 5 & 14.41 & 14.36 & 15.01 & 15.60 & 15.6 \\
\hline 6 & 10.50 & 12.40 & 11.76 & 11.15 & 12.6 \\
\hline 7 & --- & --- & 15.31 & --- & --- \\
\hline \multicolumn{6}{|c|}{ Minor Groove Depth $(\AA)$} \\
\hline Base \# & CG & $\mathrm{CG}^{8-\mathrm{Ph}}$ & $\mathrm{CG}^{8 \mathrm{-Tol}}$ & $\mathrm{CG}^{8-\mathrm{Hmp}}$ & CG* \\
\hline 2 & 4.49 & 4.60 & 4.37 & 4.52 & 4.2 \\
\hline 3 & 5.02 & 5.25 & 4.89 & 2.75 & 5.1 \\
\hline 4 & 4.62 & 4.20 & 4.27 & 4.87 & 4.2 \\
\hline 5 & 5.07 & 2.91 & 2.70 & 5.16 & 5.1 \\
\hline 6 & 4.10 & 3.96 & 4.18 & 4.23 & 4.4 \\
\hline 7 & 2.55 & 5.19 & 5.04 & 5.28 & 5.1 \\
\hline \multicolumn{6}{|c|}{ Major Groove Depth $(\AA)$} \\
\hline Base \# & CG & $\mathrm{CG}^{8-\mathrm{Ph}}$ & $\mathrm{CG}^{8 \mathrm{Tol}}$ & $\mathrm{CG}^{8-\mathrm{Hmp}}$ & $\mathrm{CG}^{*}$ \\
\hline 2 & -- & -- & --- & --- & --- \\
\hline 3 & -3.18 & --- & --- & -3.63 & --- \\
\hline 4 & -1.47 & -1.79 & -0.24 & -2.85 & -3.5 \\
\hline 5 & $\mathbf{0 . 5 7}$ & 0.46 & -0.29 & -0.61 & 0.0 \\
\hline 6 & $\begin{array}{r}-3.39 \\
\end{array}$ & -1.50 & -2.05 & -0.94 & -2.0 \\
\hline 7 & --- & --- & -2.54 & --- & --- \\
\hline
\end{tabular}

${ }^{\text {a }}$ Canonical Z-DNA as calculated by CURVES. 
Table 5.9a. Diameter of unmodified and modified duplexes in the B-DNA conformation.

\begin{tabular}{|c|c|c|c|c|c|}
\hline \multicolumn{6}{|c|}{ Diameter $(\mathbf{\AA})$} \\
\hline Base \# & $\mathbf{C G}$ & $\mathbf{C G}^{\text {8-Ph }}$ & $\mathbf{C G}^{\text {8-Tol }}$ & $\mathbf{C G}^{\text {8-Hmp }}$ & $\mathbf{C G}^{\mathbf{a}}$ \\
\hline 2 & 20.17 & 18.99 & 19.76 & 18.50 & 17.8 \\
\hline 3 & 20.95 & 19.62 & 18.79 & 18.70 & 17.8 \\
\hline 4 & 20.25 & 20.58 & 18.65 & 18.21 & 17.8 \\
\hline 5 & $\mathbf{1 9 . 8 5}$ & $\mathbf{1 9 . 5 5}$ & $\mathbf{1 8 . 2 6}$ & $\mathbf{1 7 . 9 7}$ & $\mathbf{1 7 . 8}$ \\
\hline $\mathbf{6}$ & $\mathbf{2 1 . 0 9}$ & $\mathbf{2 0 . 1 7}$ & $\mathbf{1 9 . 5 2}$ & $\mathbf{1 8 . 7 8}$ & $\mathbf{1 7 . 8}$ \\
\hline 7 & 20.08 & 19.81 & 19.35 & 18.81 & 17.8 \\
\hline 8 & 20.02 & 20.06 & 19.83 & 18.63 & 17.8 \\
\hline
\end{tabular}

${ }^{\mathrm{a} C a n o n i c a l ~ B-D N A}$ as calculated by CURVES.

Table 5.9b. Diameter of unmodified and modified duplexes in the Z-DNA conformation.

\begin{tabular}{|c|c|c|c|c|c|}
\hline \multicolumn{6}{|c|}{ Diameter $(\mathbf{\AA})$} \\
\hline Base \# & $\mathbf{C G}$ & $\mathbf{C G}^{\text {8-Ph }}$ & $\mathbf{C G}^{\text {8-Tol }}$ & $\mathbf{C G}^{\text {8-Hmp }}$ & $\mathbf{C G}^{\mathbf{a}}$ \\
\hline 2 & 16.34 & 16.74 & 16.71 & 16.21 & 14 \\
\hline 3 & 14.85 & 14.03 & 14.05 & 13.88 & 14 \\
\hline 4 & 16.14 & 15.91 & 16.47 & 16.03 & 14 \\
\hline 5 & $\mathbf{1 3 . 4 9}$ & $\mathbf{1 3 . 8 8}$ & $\mathbf{1 3 . 9 7}$ & $\mathbf{1 4 . 0 9}$ & 14 \\
\hline $\mathbf{6}$ & $\mathbf{1 6 . 4 7}$ & $\mathbf{1 6 . 3 2}$ & $\mathbf{1 6 . 9 0}$ & $\mathbf{1 6 . 0 9}$ & $\mathbf{1 4}$ \\
\hline 7 & 13.21 & 13.84 & 14.40 & 14.02 & 14 \\
\hline 8 & 16.40 & 16.40 & 16.35 & 16.66 & 14 \\
\hline
\end{tabular}

${ }^{a}$ Canonical Z-DNA as calculated by CURVES.

a)

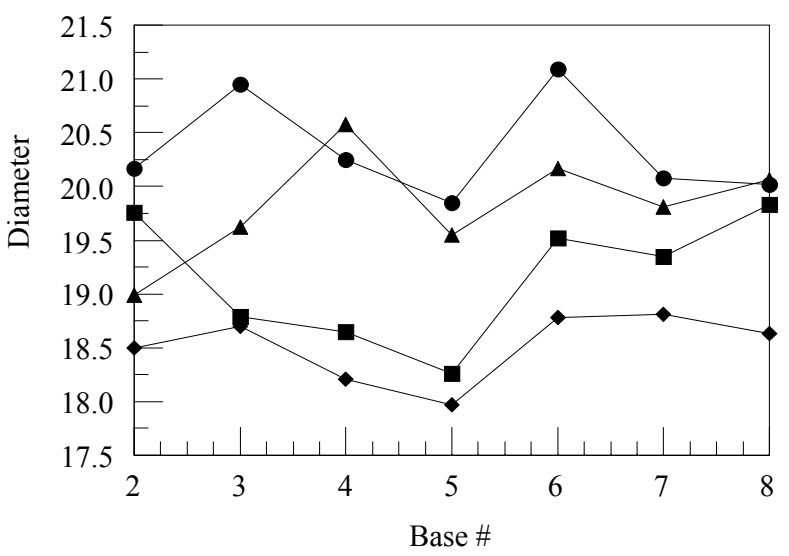

b)

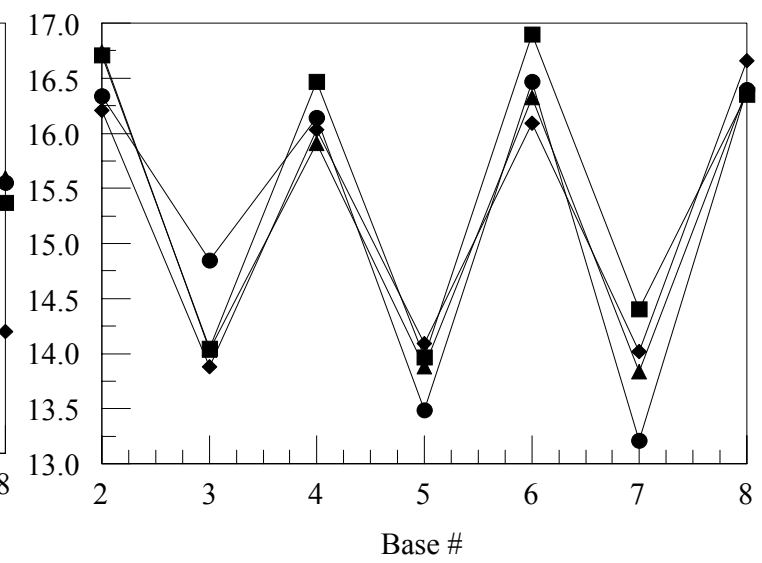

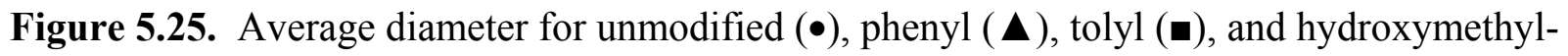
phenyl ( $\bullet$ modified a) B and b) Z-DNA's. 
The diameter of the modified B-DNA's is smaller overall than the unmodified, and this difference is most notable at the $5^{\text {th }}$ and $6^{\text {th }}$ base pairs, as shown in Figure 5.25, correlating to the modification at G6 and G16. $\mathbf{C G}^{\mathbf{8 - H m p}}$ is smallest in diameter, followed by $\mathbf{C G}^{\mathbf{8 - T o l}}$ and $\mathbf{C G}^{\mathbf{8 - P h}}$. On the other hand, there is no significant difference in the modified Z-DNA's when compared to the unmodified. This difference is also observable in Table 5.10, which show no significant shortening in the modified Z-DNA's, but considerable shortening in the tolyl-modified B-DNA and to a lesser extent in the hydroxymethphenyl and phenyl-modified DNA's in comparison with the unmodified. Shortening is an indication of curvature, i.e., the actual path length from end-toend is longer than the direct path from end-to-end. The modified B-DNA's are also significantly longer (actual path length) than their unmodified counterpart.

Table 5.10a. Global axis parameters for unmodified and modified duplexes in the B-DNA conformation.

\begin{tabular}{|l|l|l|l|l|l|}
\hline Curvature & $\mathbf{C G}$ & $\mathbf{C G}^{8-\mathbf{P h}}$ & $\mathbf{C G}^{8-\mathbf{T o l}}$ & $\mathbf{C G}^{\text {8-Hmp }}$ & $\mathbf{C G}^{\mathbf{a}}$ \\
\hline Actual Path Length & 28.83 & 32.35 & 34.82 & 33.10 & 30.41 \\
\hline Direct Path Length & 28.18 & 31.49 & 32.35 & 31.77 & 30.41 \\
\hline Shortening & $2.25 \%$ & $2.65 \%$ & $7.05 \%$ & $4.02 \%$ & $0.0 \%$ \\
\hline
\end{tabular}

${ }^{a}$ Canonical B-DNA as calculated by CURVES.

Table 5.10b. Global axis parameters for unmodified and modified duplexes in the Z-DNA conformation.

\begin{tabular}{|l|l|l|l|l|l|}
\hline Curvature & CG & $\mathbf{C G}^{\text {8-Ph }}$ & $\mathbf{C G}^{\text {8-Tol }}$ & $\mathbf{C G}^{\text {8-Hmp }}$ & $\mathbf{C G}^{\mathbf{a}}$ \\
\hline Actual Path Length & 32.94 & 32.89 & 33.33 & 32.04 & 33.88 \\
\hline Direct Path Length & 32.81 & 32.83 & 33.05 & 31.94 & 33.88 \\
\hline Shortening & $0.41 \%$ & $0.17 \%$ & $0.83 \%$ & $0.31 \%$ & $0.00 \%$ \\
\hline
\end{tabular}

${ }^{a}$ Canonical Z-DNA as calculated by CURVES.

Figures 5.26 and 5.27 depict the torsion angles for the most representative B and Z-DNA structures. All structures show some deviation from canonical values, but the variation in torsion angles for the modified DNA's relative to the unmodified is similar for both the B and Z-DNA conformations. The bimodal distribution found for alpha, gamma, zeta, and chi are consistent 
with Z-DNA (refer to Table 5.7). 


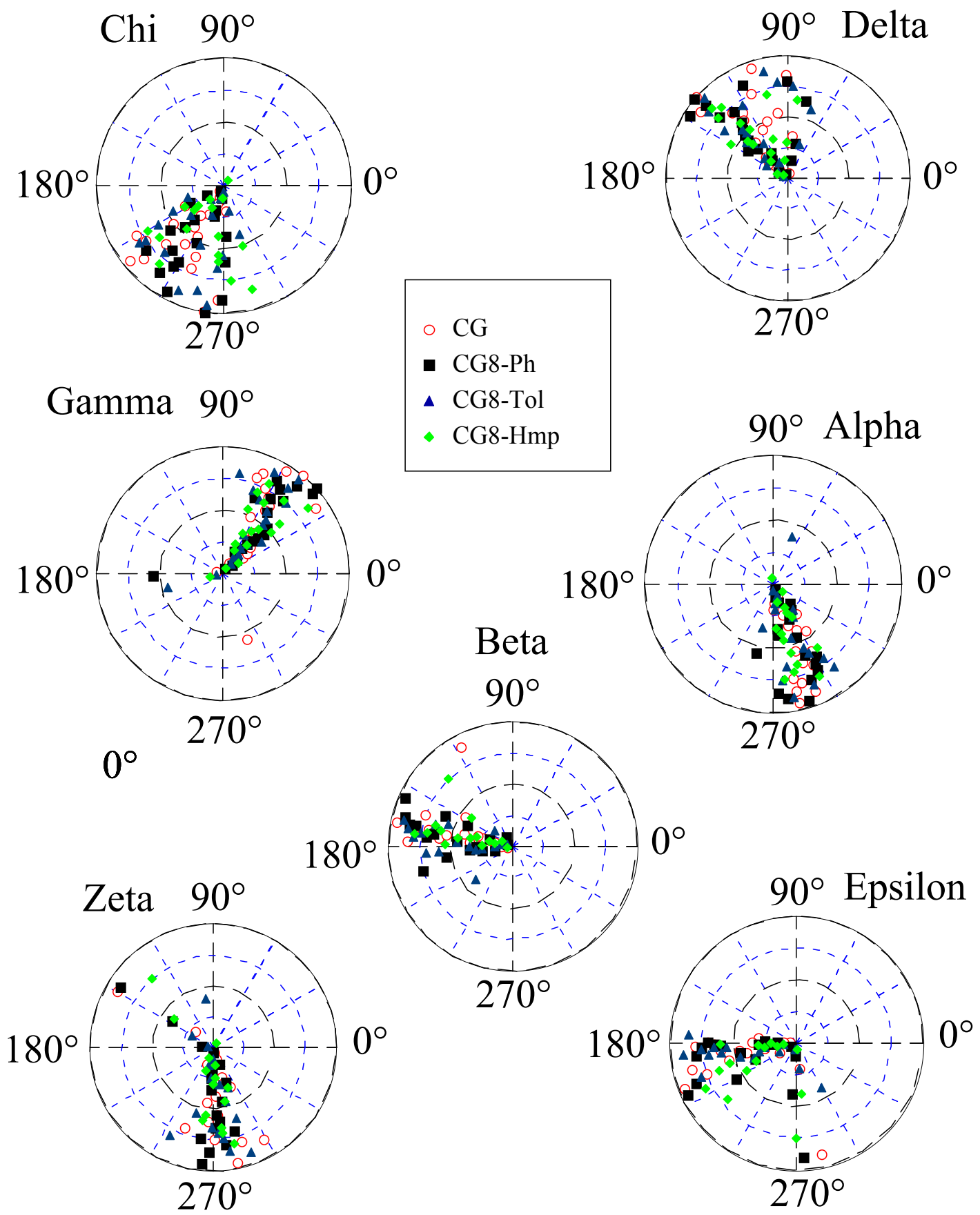

Figure 5.26. Torsion angles for the most representative B-DNA structures. Values are for bases 1-20 with each grid representing five bases each. The values near 1, 10, 11, and 20 may be weird due to end effects. 


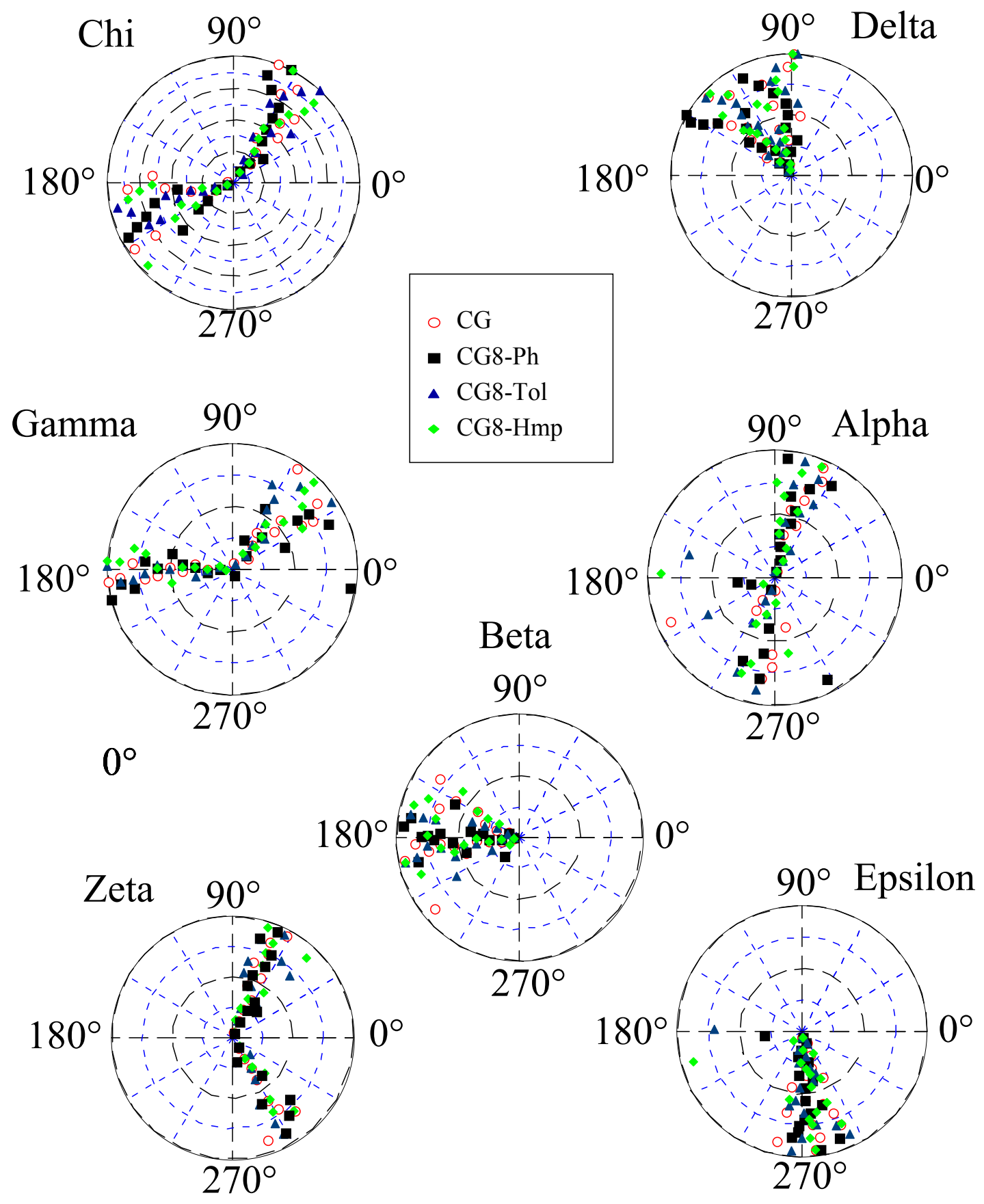

Figure 5.27. Torsion angles for the most representative Z-DNA structures. Values are for bases 1-20 with each grid representing five bases each. The values near 1, 10, 11, and 20 may be weird due to end effects. 
The global structural parameters shown in Figure 5.28 are defined as shifts in the direction of or rotation around the axes depicted in Figure 5.24. ${ }^{138}$ The rotational and translational movements can involve two bases of a pair or two successive base pairs of a step, and they can be co-coordinated or opposed. The motions involving base pairs include tip (TIP), inclination (INC), opening (OPN), propeller twist (PRP), buckle (BKL), x (XDP) and y (YDP) displacement, stagger (STG), stretch (STR), and shear (SHR). The motions involving successive bases include rise (RIS), slide (SLD), shift (SHF), twist (TWT), roll (ROL), and tilt (TLT);

Figure 5.28 illustrates them. Table 5.11 gives the definitions and names for the helical parameters; the "base pair value" column indicates how compound parameters are built up from underlying parameters referring to individual bases. 
Table 5.11. Helicoidal parameter names and definitions for base pair values. ${ }^{145}$

\begin{tabular}{|c|c|c|c|c|}
\hline Name & Family & Code & Symbol & $\begin{array}{l}\text { Base Pair } \\
\text { Value } \delta\end{array}$ \\
\hline X-displacement & Base pair axis & XDP & $\overline{d x}$ & 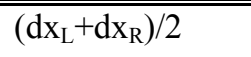 \\
\hline Y-displacement & & YDP & dy & $\left(\mathrm{dy}_{\mathrm{L}}-\mathrm{dy}_{\mathrm{R}}\right) / 2$ \\
\hline Inclination & & INC & $\eta$ & $\left(\eta_{L}+\eta_{R}\right) / 2$ \\
\hline Tip & & TIP & $\theta$ & $\left(\theta_{\mathrm{L}}-\theta_{\mathrm{R}}\right) / 2$ \\
\hline Shear & Intra-base pair & SHR & $\mathrm{S}_{\mathrm{x}}$ & $d x_{L}-d x_{R}$ \\
\hline Stretch & & STR & $\mathrm{S}_{\mathrm{y}}$ & $\mathrm{dy}_{\mathrm{L}}+\mathrm{dy}_{\mathrm{R}}$ \\
\hline Stagger & & STG & $\mathrm{S}_{\mathrm{z}}$ & $\sum_{m=1}^{i}\left(\mathrm{Dz}_{\mathrm{L}}+\mathrm{Dz} \mathrm{z}_{\mathrm{R}}\right)$ \\
\hline Buckle & & BKL & $\kappa$ & $\eta_{L}+\eta_{R}$ \\
\hline Propeller & & PRP & $\omega$ & $\left(\theta_{\mathrm{L}}-\theta_{\mathrm{R}}\right)$ \\
\hline Opening & & OPN & $\alpha$ & $\sum_{m=1}^{i}\left(\Omega_{\mathrm{L}}+\Omega_{\mathrm{R}}\right)$ \\
\hline Shift & Inter-base pair & SHF & $\mathrm{D}_{\mathrm{x}}$ & $\mathrm{dx}(\mathrm{i})+\mathrm{A}_{\mathrm{x}}-\mathrm{dx}(\mathrm{i}-1)$ \\
\hline Slide & & SLD & $\mathrm{D}_{\mathrm{y}}$ & $d y(i)+A_{y}-d y(i-1)$ \\
\hline Rise & & RIS & $\mathrm{D}_{\mathrm{z}}$ & \\
\hline Tilt & & TLT & $\tau$ & $\eta(i)+\eta_{A}-\eta(i-1)$ \\
\hline Roll & & ROL & $\rho$ & $\theta(\mathrm{i})+\theta_{\mathrm{A}}-\theta(\mathrm{i}-1)$ \\
\hline Twist & & TWT & $\Omega$ & \\
\hline Axis X-displacement & Axis & AXD & $\mathrm{A}_{\mathrm{x}}$ & \\
\hline Axis Y-displacement & & AYD & $\mathrm{A}_{\mathrm{y}}$ & \\
\hline Axis inclination & & AIN & $\eta_{\mathrm{A}}$ & \\
\hline Axis tip & & ATP & $\theta_{\mathrm{A}}$ & \\
\hline
\end{tabular}



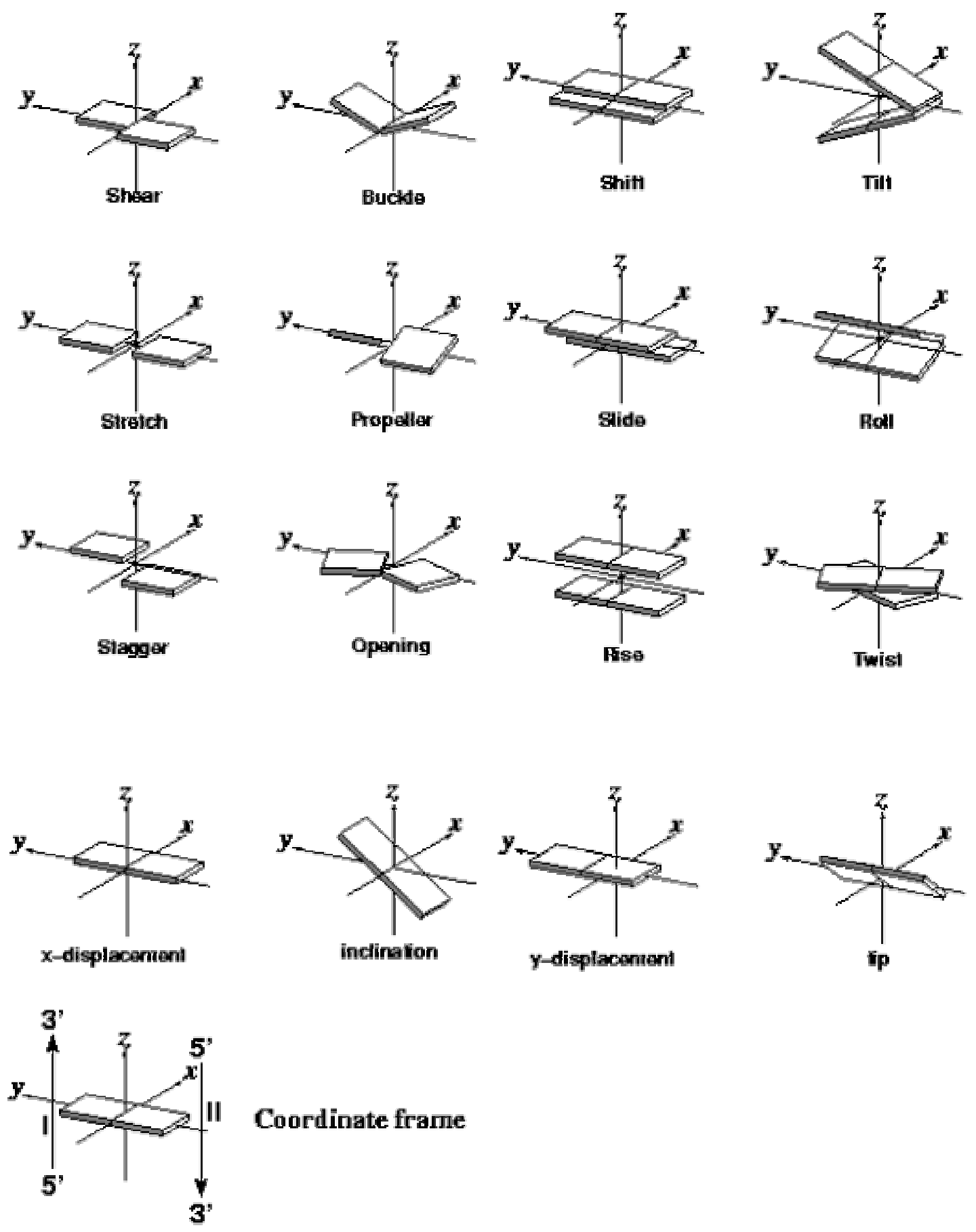

Figure 5.28. Diagrams illustrating movements in the positive direction of base pairs or successive bases. ${ }^{143}$ 
Figures 5.29, 5.30, and 5.31 show the inter-base pair, base pair-axis, and intra-base pair parameters, respectively, over the course of the 2 ns trajectory for the B-DNA's generated from Dials and Windows. ${ }^{137,139}$ Slide for the G6-C15 base pair is significantly negative for all modifications, resulting in the phenyl of G6 stacking over C5 and the phenyl of G16 stacking under C15. Although the degree of difference in slide is the same for all modifications, the phenyl in the HMP-modified guanines is not directly above the cytosine (in the $3^{\prime}$ to $5^{\prime}$ direction) as seen in the other modifications. This is because the phenyl is orientated in such a way as to minimize the distance between the hydrogen of the hydroxy group and its own O2P oxygen. This distance for both G6 and G16 is plotted in Figure 5.32. The phenyl and tolyl modifications have a more positive rise relative to the unmodified B-DNA; however, the difference in rise in $\mathbf{C G}^{\mathbf{8}-\mathrm{Hmp}}$ relative to the unmodified is less than the other adducts. This is also due to the lesser degree of stacking of the phenyl group for the HMP modification. In the phenyl and tolylmodified DNA's, the degree of stacking is similar to that found in intercalating agents, which result in an increase in rise. ${ }^{146}$ Twist is less for all modified oligos relative to the unmodified BDNA, but variability among all base pairs (modified DNA's) for this parameter makes it difficult to assess the significance. Also affected by the modifications are the base pair-axis parameters, in particular inclination, which goes from positive to negative as one moves down the base pairs to the modification and then goes back to positive after the modification. Inclination has been related to minor groove width in an inversely proportional manner, ${ }^{147}$ but no such correlation is observed in this work. The intra-base pair parameters are largely unaffected by the modification in the B-DNA conformation. 
a) $\mathbf{C G}$

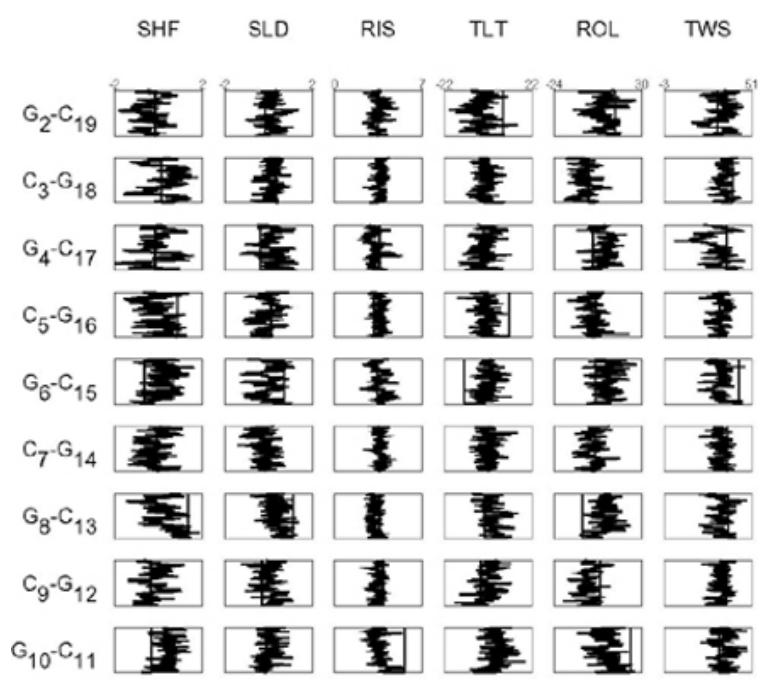

c) $\quad \mathrm{CG}^{8-\mathrm{Tol}}$

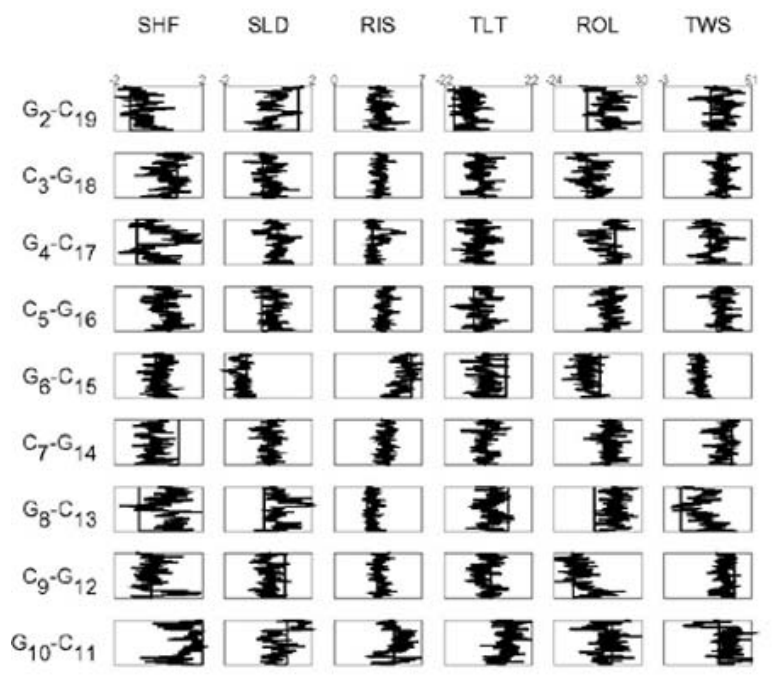

b) $\quad \mathbf{C G}^{8-\mathrm{Ph}}$

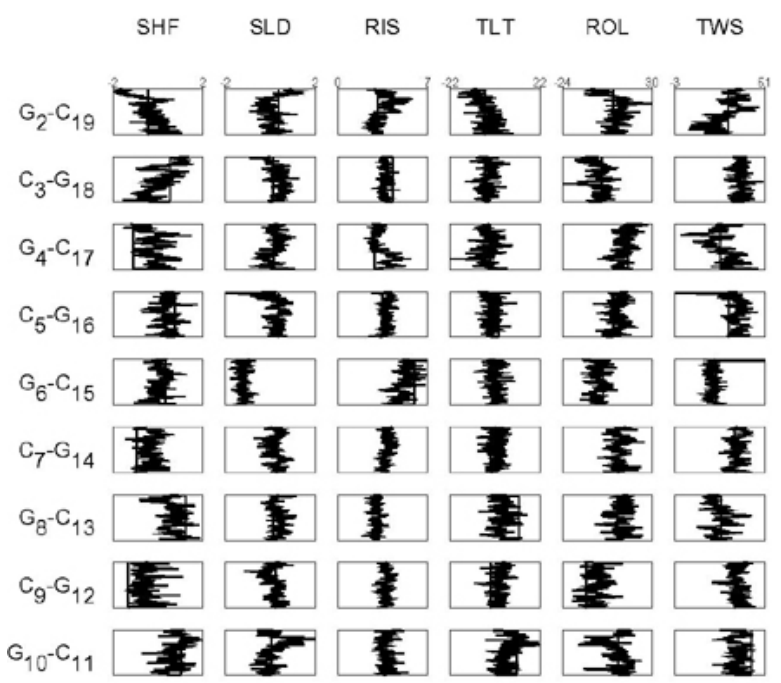

d) $\quad \mathrm{CG}^{8-\mathrm{Hmp}}$

Figure 5.29. Shift (-2 to $2 \AA)$, slide ( -2 to $2 \AA)$, rise $\left(0\right.$ to $7 \AA$ ), tilt( $\left(-22\right.$ to $\left.22^{\circ}\right)$, roll $\left(-24\right.$ to $\left.30^{\circ}\right)$, and twist $\left(-3\right.$ to $\left.51^{\circ}\right)$ of base pairs over the course of the 2 ns production trajectory for a) $\left.\mathbf{C G}, b\right)$ $\mathbf{C G}^{\mathbf{8 - P h}}$, c) $\mathbf{C G}^{\mathbf{8 - T o l}}$, and d) $\mathbf{C G}^{\mathbf{8 - H m p}}$ in the B-DNA conformation. Ranges, given in parentheses after each parameter, are identical in a-d. 
a) $\mathbf{C G}$

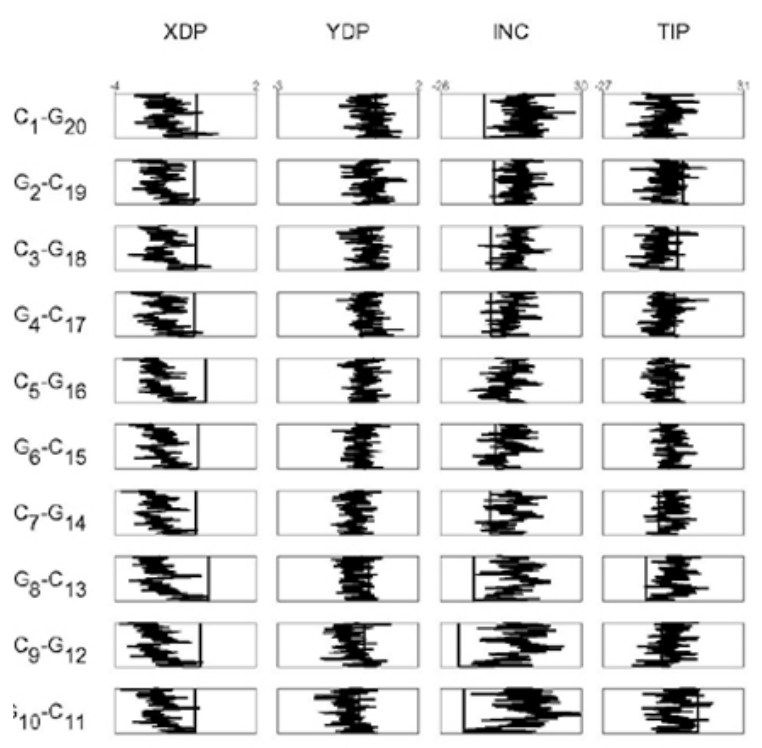

b) $\quad \mathbf{C G}^{8-\mathrm{Ph}}$

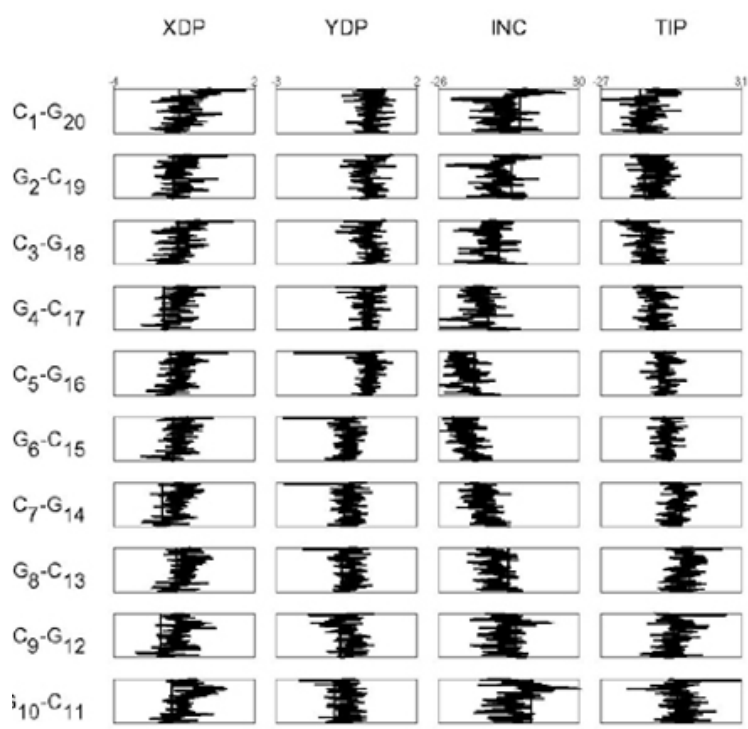

c) $\quad \mathrm{CG}^{8-\mathrm{Tol}}$

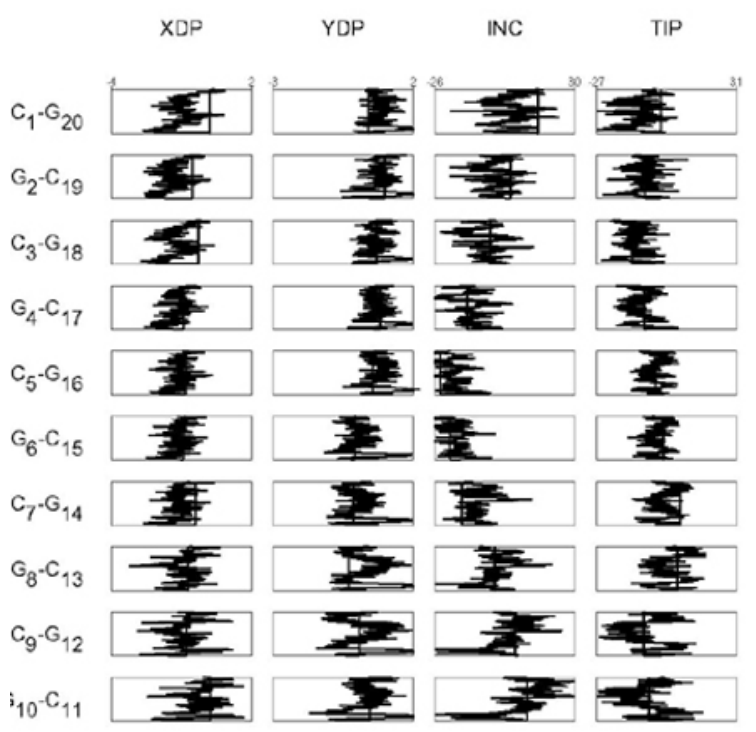

d) $\quad \mathrm{CG}^{8-\mathrm{Hmp}}$

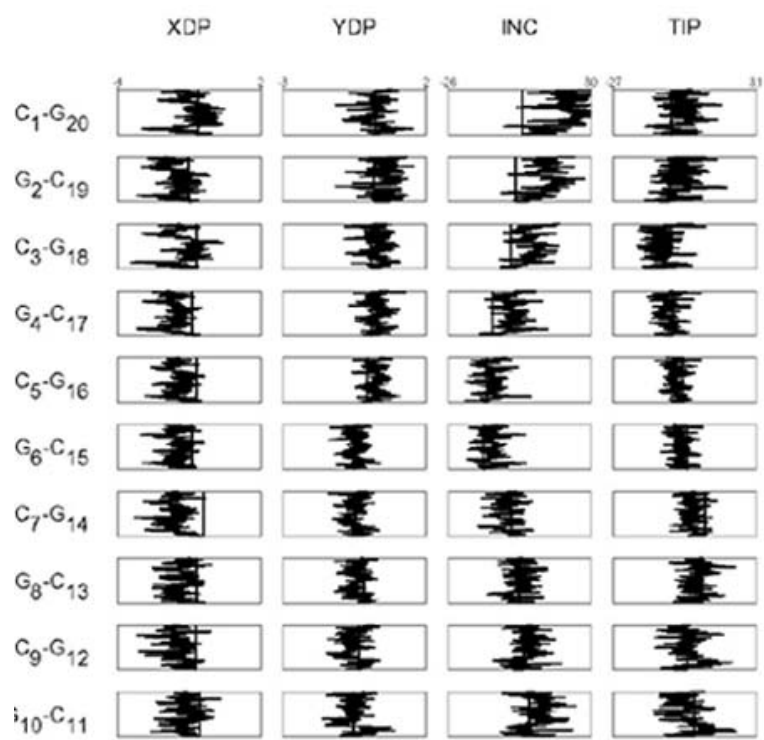

Figure 5.30. X-displacement (-4 to $2 \AA$ ), y-displacement (-3 to $2 \AA)$, inclination ( -26 to $30^{\circ}$ ), and tip $\left(-27\right.$ to $\left.31^{\circ}\right)$ of base pairs over the course of the 2 ns production trajectory for a) $\mathbf{C G}, \mathrm{b}$ ) $\mathbf{C G}^{\mathbf{8 - P h}}$, c) $\mathbf{C G}^{\mathbf{8 - T o l}}$, and d) $\mathbf{C G}^{\mathbf{8 - H m p}}$ in the B-DNA conformation. Ranges, given in parentheses after each parameter, are identical in a-d. 
a) CG

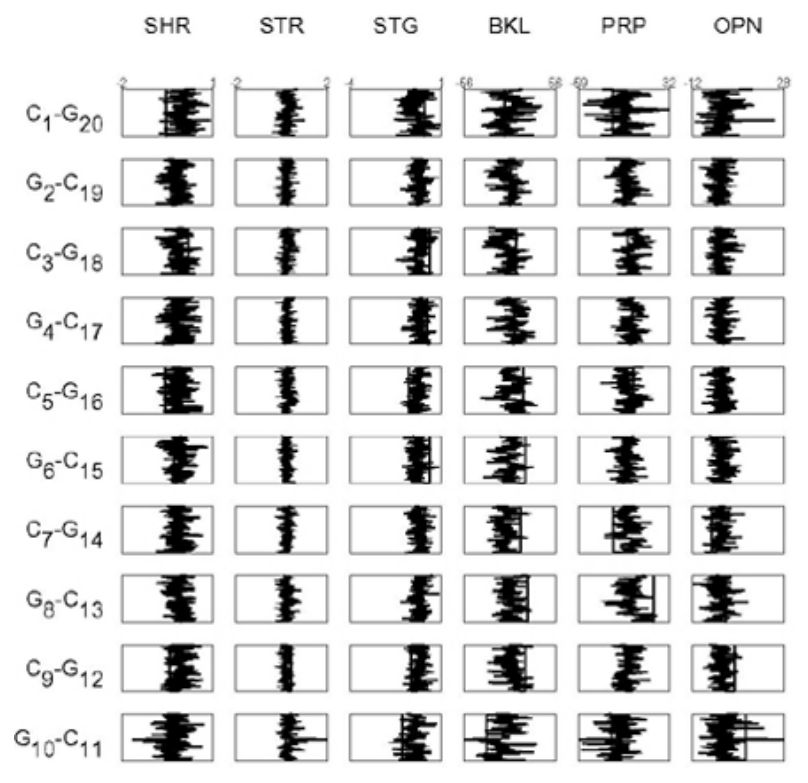

c) $\quad \mathrm{CG}^{8-\mathrm{Tol}}$

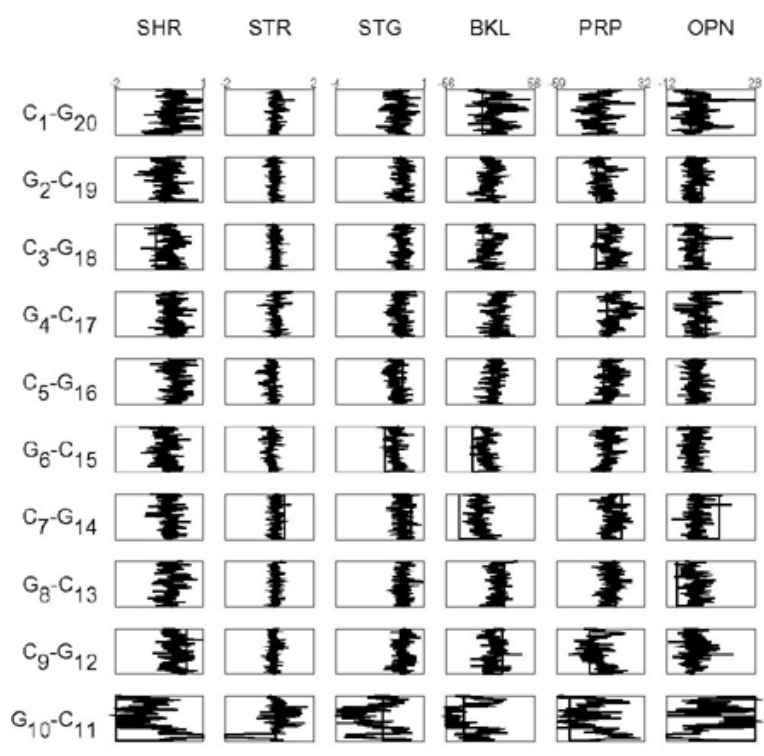

b) $\quad \mathbf{C G}^{\mathbf{8 - P h}}$

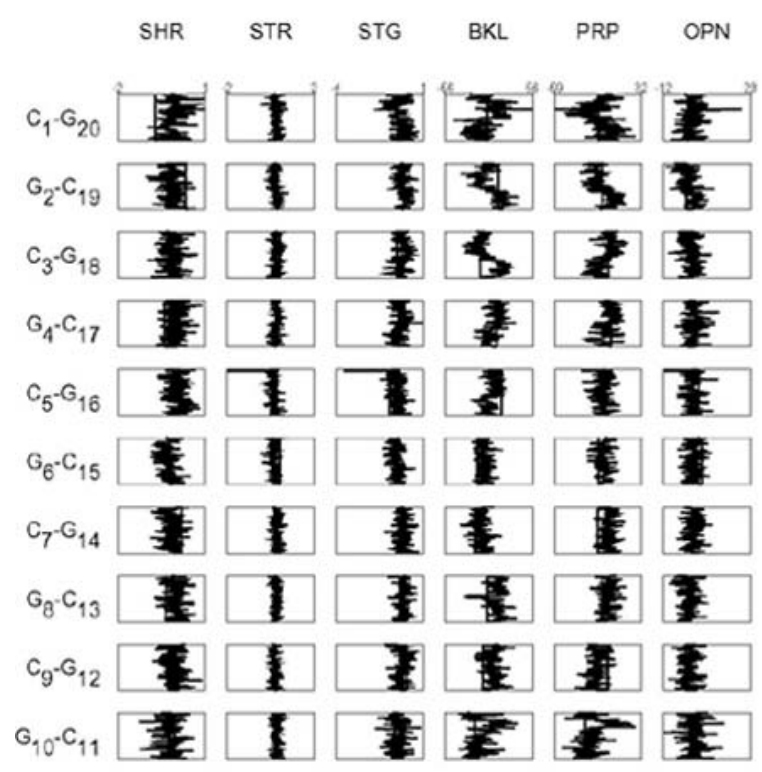

d) $\quad \mathbf{C G}^{8-\mathrm{Hmp}}$

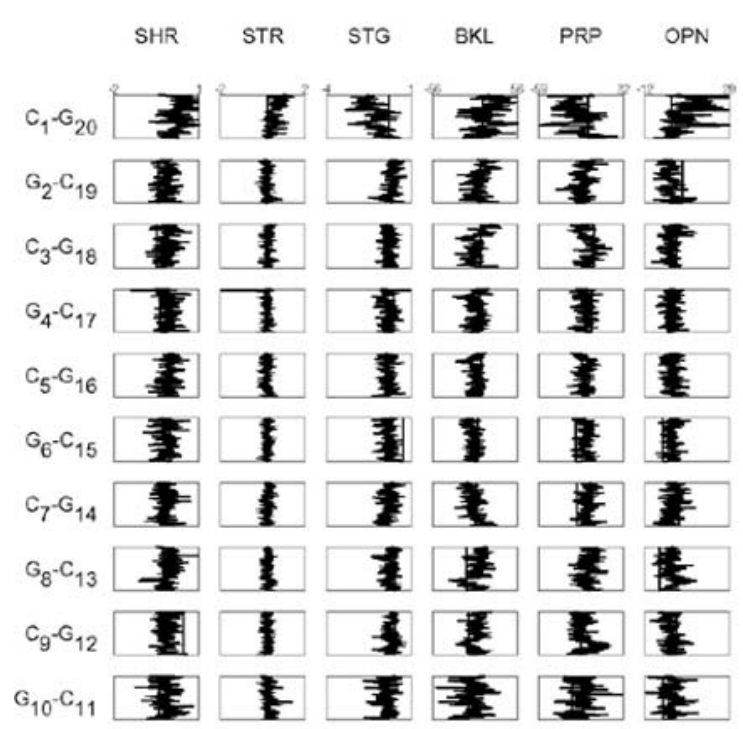

Figure 5.31. Shear ( -2 to $1 \AA)$, stretch ( -2 to $2 \AA$ ), stagger ( -4 to $1 \AA)$, buckle (-56 to $\left.56^{\circ}\right)$, propeller $\left(-59\right.$ to $\left.32^{\circ}\right)$, and opening $\left(-12\right.$ to $\left.28^{\circ}\right)$ of base pairs over the course of the $2 \mathrm{~ns}$ production trajectory for a) $\mathbf{C G}$, b) $\mathbf{C G}^{\mathbf{8 - P h}}$, c) $\mathbf{C G}^{\mathbf{8 - T o l}}$, and d) $\mathbf{C G}^{\mathbf{8 - H m p}}$ in the B-DNA conformation. Ranges, given in parentheses after each parameter, are identical in a-d. 


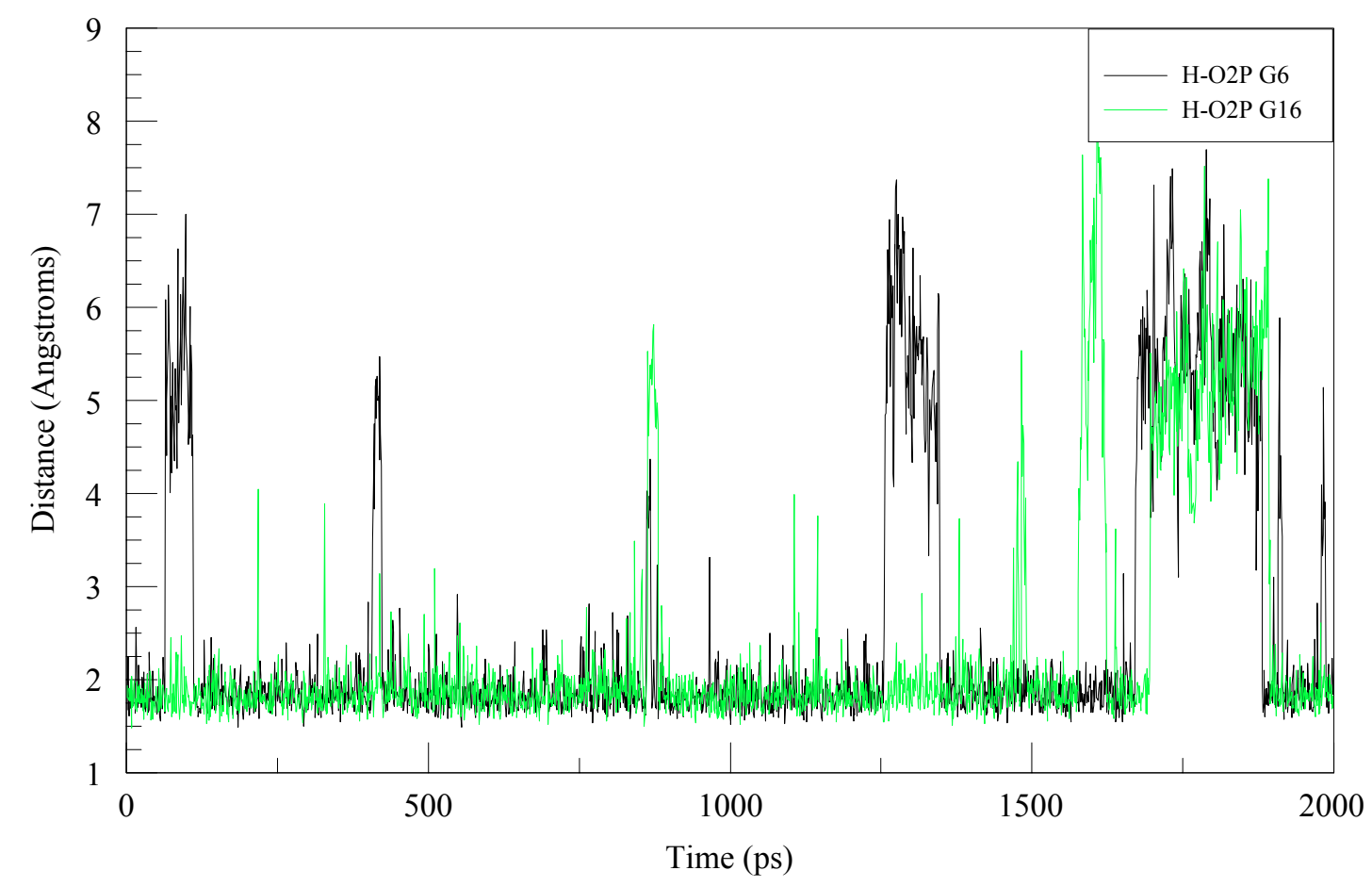

Figure 5.32. Distance between the hydrogen of the hydroxy moiety of HMP in G6 and G16 and the bases' own 02P oxygen over the course of the 2 ns production run.

Figures 5.33, 5.34, and 5.35 show the inter-base pair, base pair-axis, and intra-base pair parameters, respectively, over the course of the 2 ns trajectory for the Z-DNA's generated from Dials and Windows. All of the Z-DNA's demonstrate the "zigzag" twist and slide values characterized by the CG and GC base-steps of Z-DNA as discussed above. The modification to G6/G16 has no noticeable affect on the helical parameters of the DNA's in the Z conformation. 
a) $\mathbf{C G}$

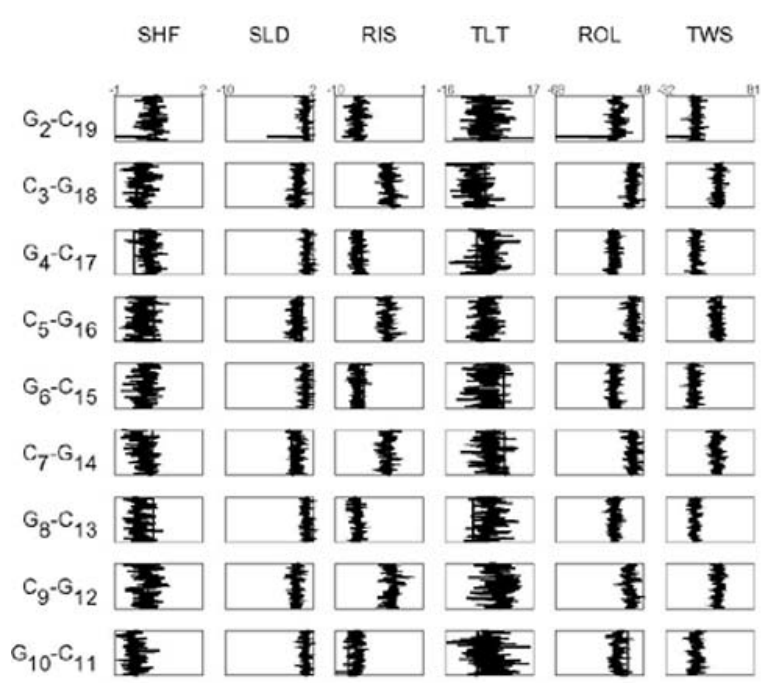

c) $\quad \mathbf{C G}^{8-\mathrm{Tol}}$

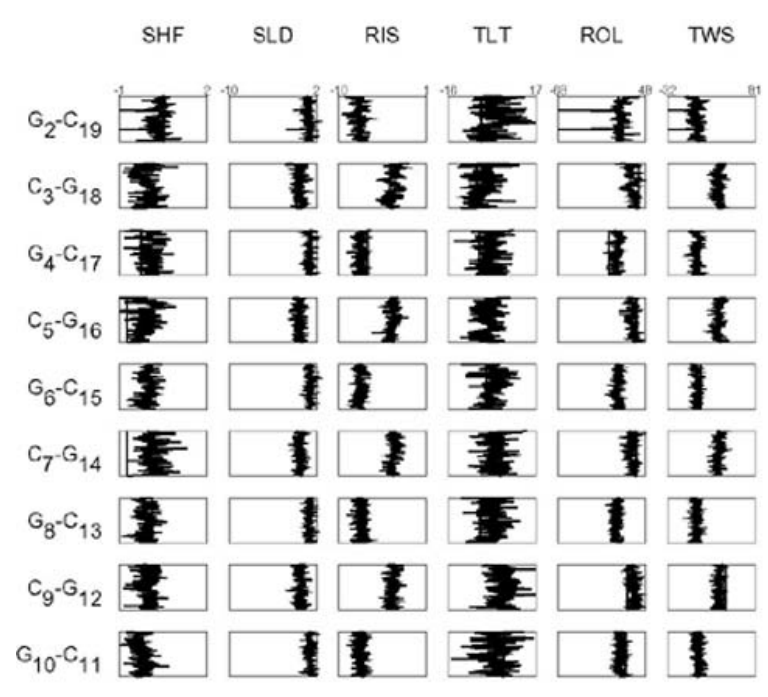

b) $\quad \mathbf{C G}^{8-\mathrm{Ph}}$

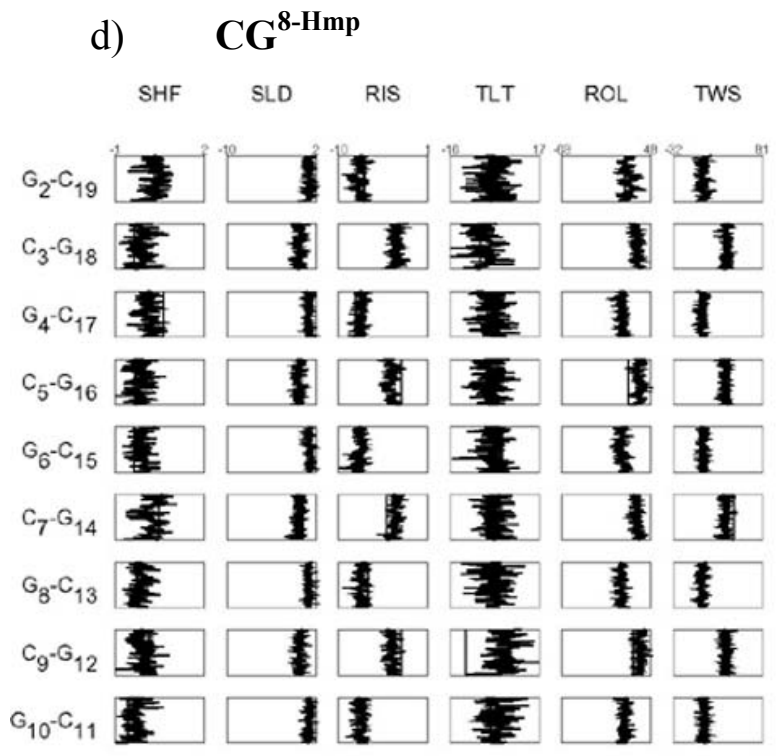

Figure 5.33. Shift (-1 to $2 \AA)$, slide ( -10 to $2 \AA)$, rise (-10 to $1 \AA)$, tilt (-16 to $\left.17^{\circ}\right)$, roll (-68 to $48^{\circ}$ ), and twist ( -32 to $81^{\circ}$ ) of base pairs over the course of the 2 ns production trajectory for a) $\mathbf{C G}$, b) $\mathbf{C G}^{\text {8-Ph }}$, c) $\mathbf{C G}^{8-\mathrm{Tol}}$, and d) $\mathbf{C G}^{\mathbf{8 - H m p}}$ in the Z-DNA conformation. Ranges, given in parentheses after each parameter, are identical in a-d. 
a) $\mathbf{C G}$

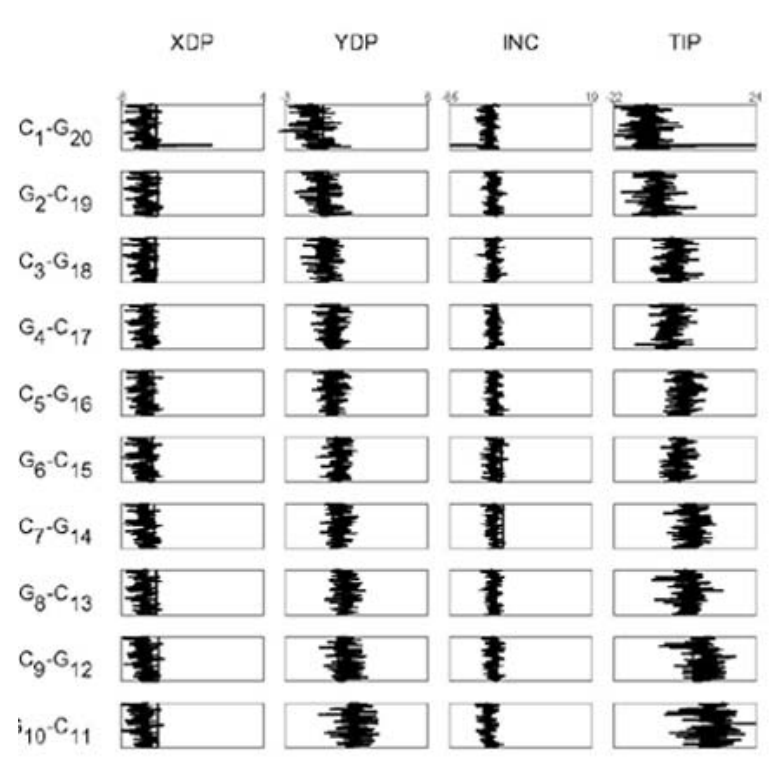

c) $\quad \mathbf{C G}^{8-\mathrm{Tol}}$

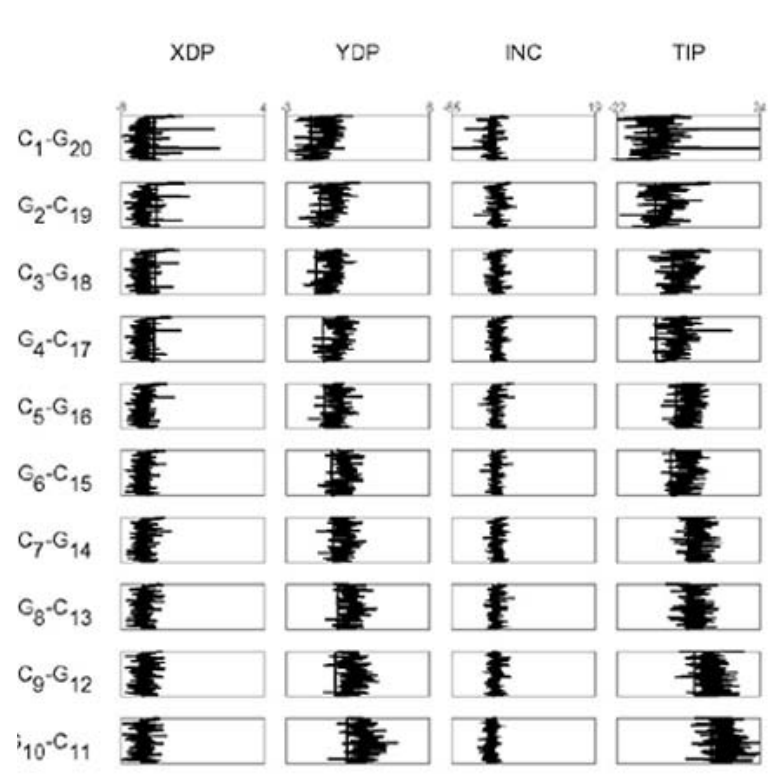

b) $\quad \mathbf{C G}^{\mathbf{8 - P h}}$

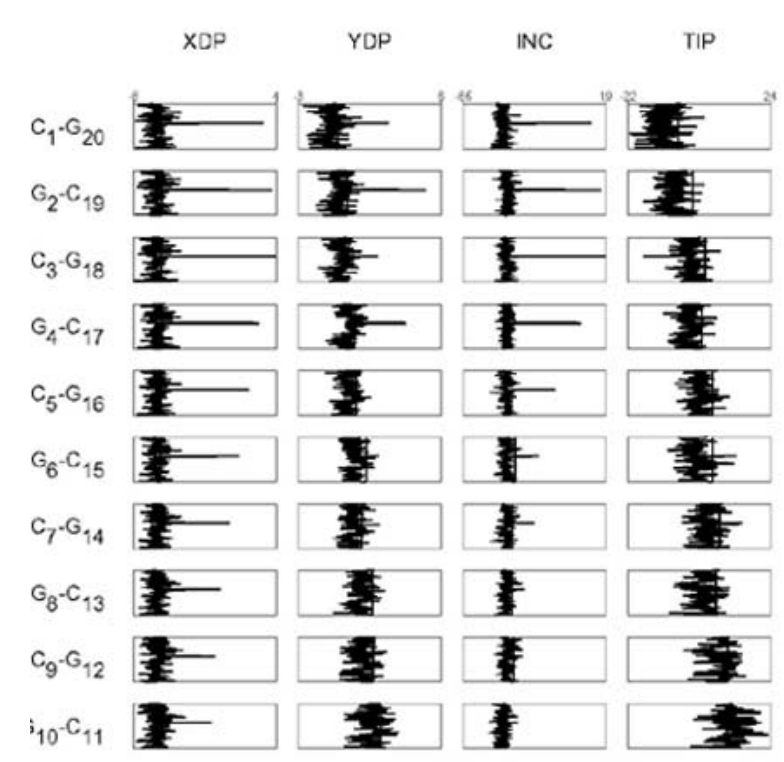

d) $\quad \mathbf{C G}^{8-\mathrm{Hmp}}$

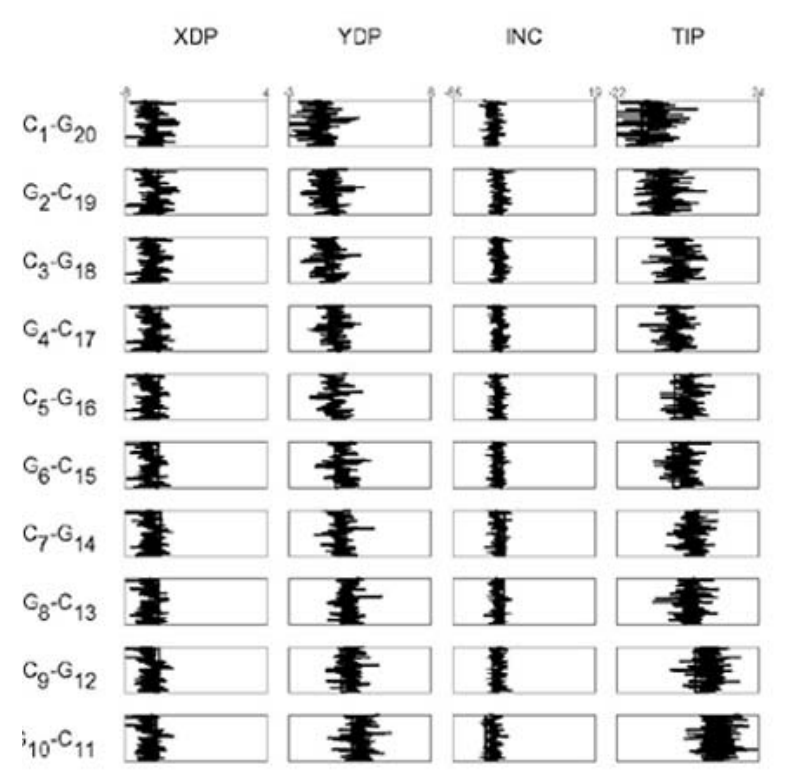

Figure 5.34. X-displacement ( -8 to $4 \AA$ ), y-displacement ( -3 to $6 \AA$ ), inclination ( -85 to $\left.19^{\circ}\right)$, and tip $\left(-22\right.$ to $\left.24^{\circ}\right)$ of base pairs over the course of the 2 ns production trajectory for a) $\mathbf{C G}, \mathrm{b}$ ) $\mathbf{C G}^{\text {8-Ph }}$, c) $\mathbf{C G}^{\text {8-Tol }}$, and d) $\mathbf{C G}^{\mathbf{8 - H m p}}$ in the Z-DNA conformation. Ranges, given in parentheses after each parameter, are identical in a-d. 
a) $\mathbf{C G}$

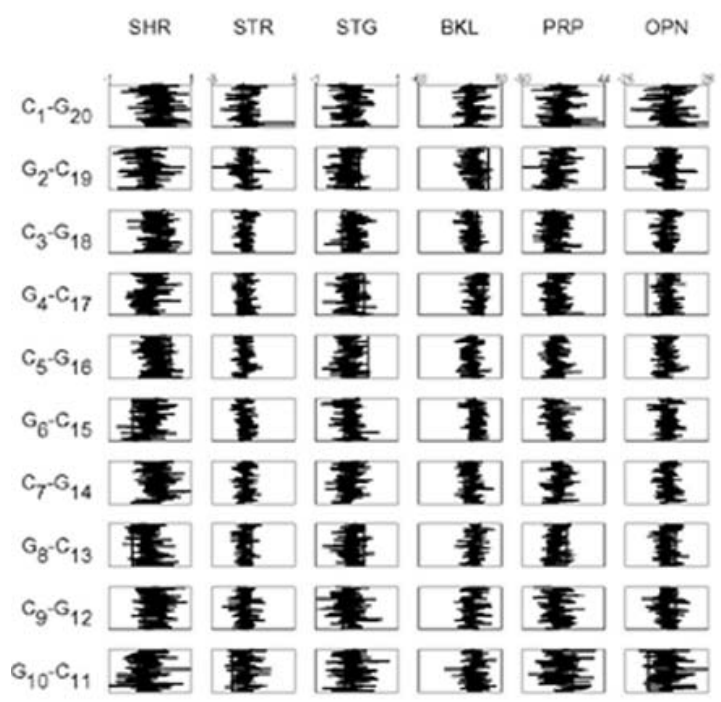

c) $\quad \mathrm{CG}^{8-\mathrm{Tol}}$

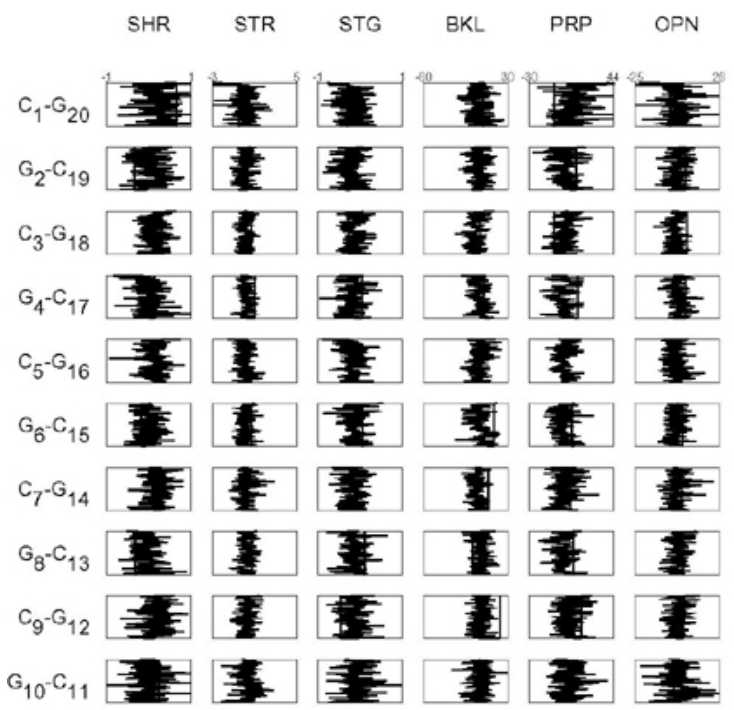

b) $\quad \mathbf{C G}^{8-\mathrm{Ph}}$

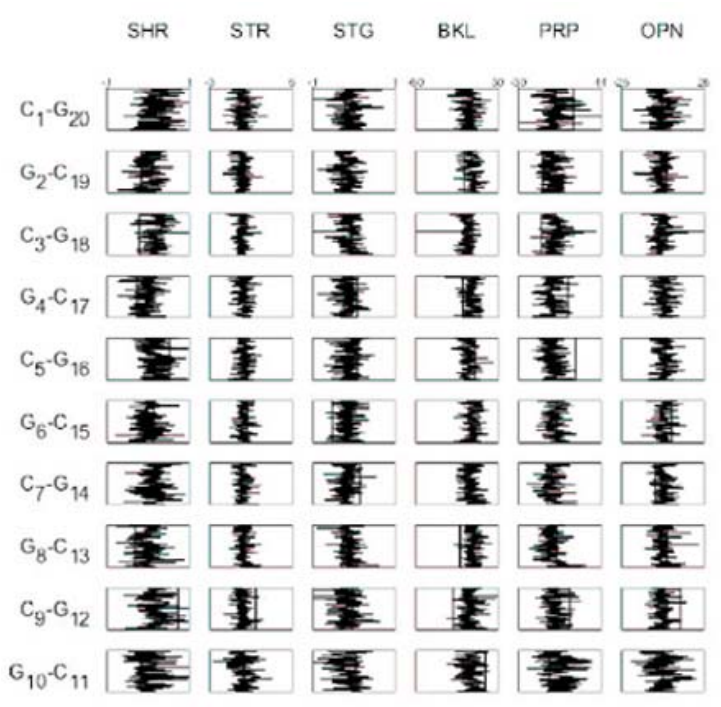

d) $\quad \mathbf{C G}^{8-\mathrm{Hmp}}$

Figure 5.35. Shear (-1 to $1 \AA$ ), stretch (-3 to $5 \AA$ ), stagger (-1 to $1 \AA$ ), buckle (-60 to $\left.30^{\circ}\right)$, propeller ( -30 to $\left.44^{\circ}\right)$, and opening $\left(-25\right.$ to $\left.26^{\circ}\right)$ of base pairs over the course of the $2 \mathrm{~ns}$ production trajectory for a) $\mathbf{C G}$, b) $\mathbf{C G}^{\mathbf{8 - P h}}$, c) $\mathbf{C G}^{\mathbf{8 - \mathrm { T } o l}}$, and d) $\mathbf{C G}^{\mathbf{8 - H m p}}$ in the Z-DNA conformation. Ranges, given in parentheses after each parameter, are identical in a-d. 
Sugar pucker is another important structural aspect to take into consideration when comparing the modified and unmodified DNA's in the B and Z-DNA conformations. Sugar pucker is commonly described by identifying the major displacement of carbons 2 ' and $3^{\prime}$ from the median plane of $\mathrm{C}^{\prime}-\mathrm{O} 4^{\prime}-\mathrm{C} 4^{\prime 143}$ but can also be described by simply designating the ring atom which puckers most out of plane with respect to the others. If the endo displacement of $\mathrm{C} 2$ ' is greater than the exo displacement of $\mathrm{C}^{2}$ ', the conformation is designated $\mathrm{C} 2$ '-endo. Exo and endo are defined relative to the face of the furanose ring; the endo face of the ring is on the same side as the base, and the exo face is opposite. The most prominent conformations, C3'-endo and C2'-endo, are illustrated in Figure 5.36. At room temperature both conformers are in dynamic equilibrium, and transitions occur via the $\mathrm{O} 4$ '-endo state. C3'-endo is found in ideal A-DNA, RNA, the purine residues of Z-DNA, and C2'-endo is found in ideal B-DNA, ${ }^{148}$ and the pyrimidine residues of Z-DNA. When changing the pucker phase angle, $\varphi$, from $0-360^{\circ}$, it is possible to step through all of the conformations of the ribose ring as shown in Table 5.12.

Table 5.12. Ranges of the sugar pucker phase angle for all conformations of the ribose; * indicates sugar puckers found for guanines in Z-DNA and **indicates puckers found in BDNA. ${ }^{148}$

\begin{tabular}{|l|l|l|}
\hline $\boldsymbol{\varphi}$ Range $\left(^{\circ}\right)$ & Name & Occurence \\
\hline$-36-0(324-360)$ & C2'-exo* & \\
\hline $0-36$ & C3'-endo* & Ideal A helix and G of Z-DNA \\
\hline $36-72$ & C4'-exo* & \\
\hline $72-108$ & O4'-endo**** & \\
\hline $108-144$ & C1'-exo*,** & \\
\hline $144-180$ & C2'-endo** & Ideal B-DNA and C of Z-DNA \\
\hline $180-216(-180 . .-144)$ & C3'-exo & \\
\hline $216-252(-144 . .-108)$ & C4'-endo & \\
\hline $252-288(-108 . .-72)$ & O4'-exo & \\
\hline $288-324(-72 . .-36)$ & C1'-endo & \\
\hline$-36-0(324-360)$ & C2'-exo & \\
\hline
\end{tabular}



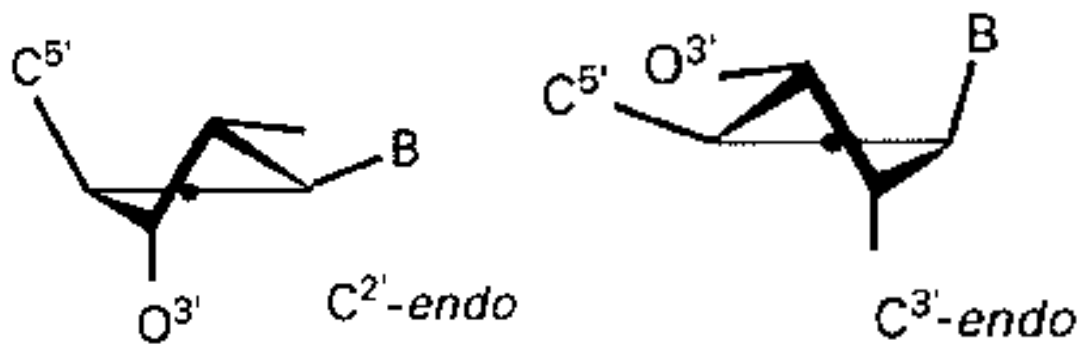

Figure 5.36. Structures of the $\mathrm{C} 2$ '-endo and $\mathrm{C} 3$ '-endo preferred sugar puckers.

Sugar puckers for the B and Z-DNA's are shown in figure 5.37, which also demonstrates the correlation between $\chi$ and $\delta$ for the B-DNA conformation. The B-DNA's displayed sugar puckers ranging from $\mathrm{C} 4$ '-exo to $\mathrm{C} 2$ '-endo. The cytosine residues of the Z-DNA's were primarily in the $\mathrm{C} 2$ '-exo conformation (with some in the range for $\mathrm{C} 2$ '-endo), and the guanines ranged from $\mathrm{C} 2^{\prime}$-exo to $\mathrm{C} 3$ 'endo. B-DNA's display the linear correlation between $\chi$ and $\delta$ as expected. ${ }^{148}$ There is no correlation between $\chi$ and $\delta$ in Z-DNA, and this is evident in the figure below. The modified guanines display sugar puckers in the $\mathrm{C} 3$ '-endo to $\mathrm{O} 1$ '-endo range; this correlates with the negative slide found in the corresponding base pair displayed in Figure 5.30. Deoxyribose sugar pucker is known to correlate with slide; ${ }^{149}$ non-zero slide helices exhibit C3'endo sugar pucker and zero slide helices exhibit C2'-endo sugar pucker. This is because sliding a base pair along the y-axis pushes the sugar ring of that base pair against the phosphate of the base below. The $\mathrm{C} 3$ '-endo sugar pucker allows the ribose ring to buckle away from the phosphate and relieves the strain. ${ }^{148}$ 
a)
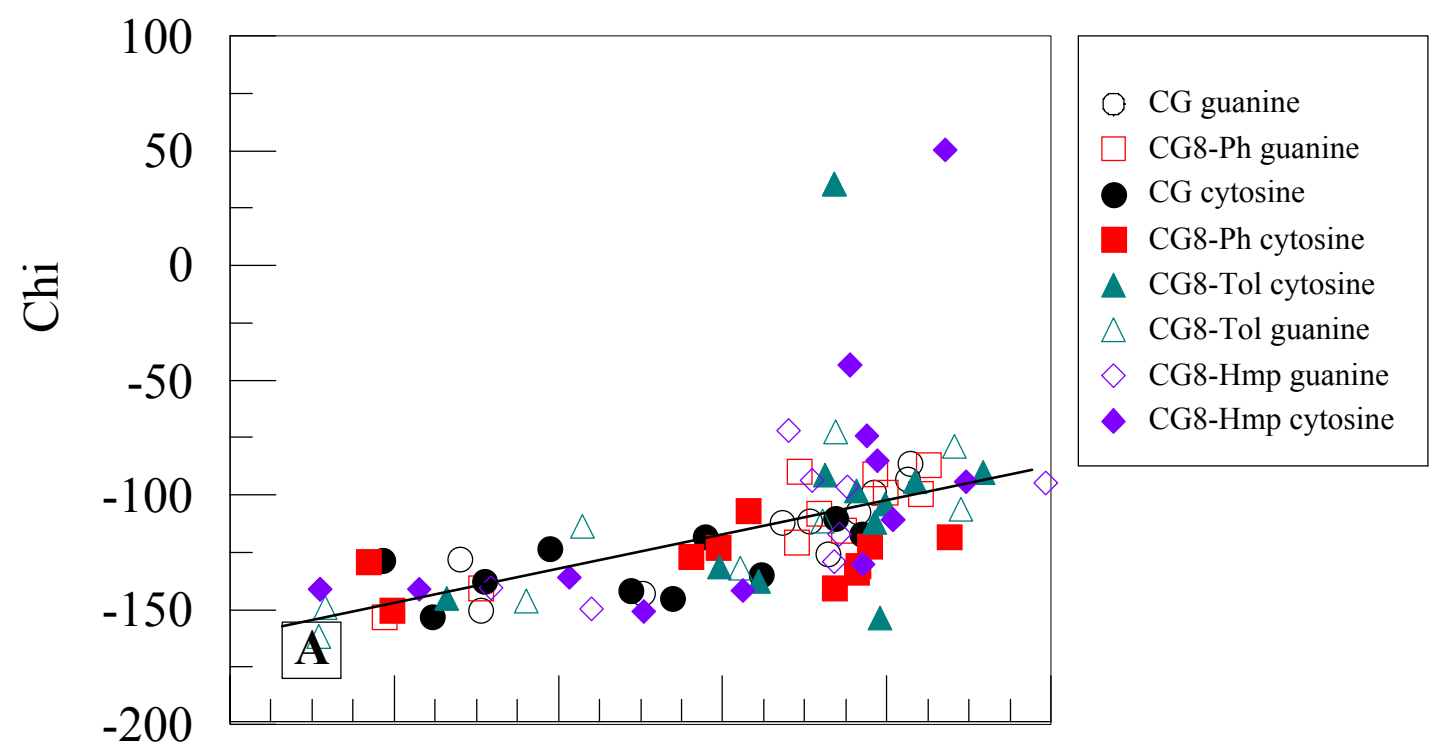

b)

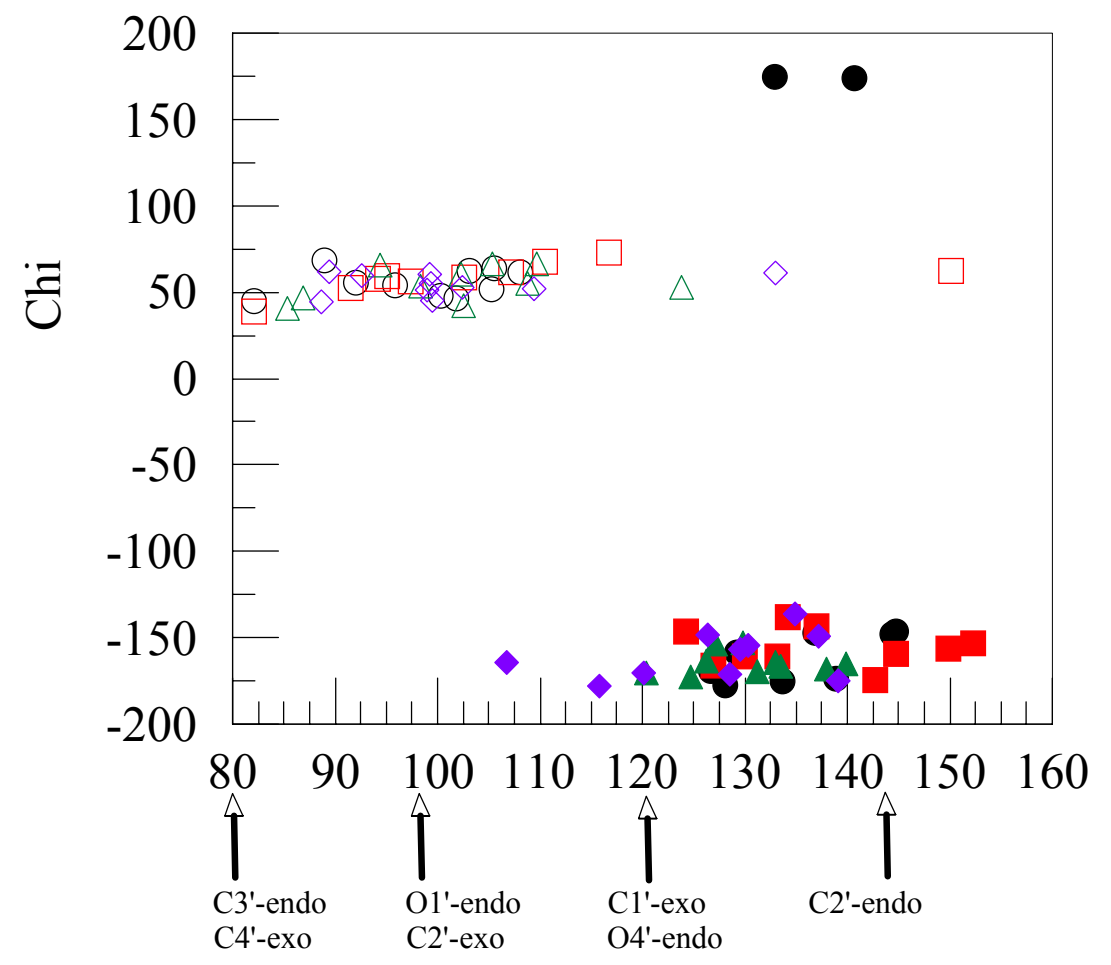

Delta

Figure 5.37. Conformation plots of $\chi$ as a function of $\delta$ for a) the B-DNA's and b) the Z-DNA's. The boxed $\mathrm{A}$ indicates the classical A helix region, and the line indicates the broad distribution of conformers found in B-DNA as established by fiber diffraction. ${ }^{148}$ 
Correlations between helical parameters calculated by CURVES and their relationship to those found in A and B-DNA fibers ${ }^{150}$ are shown in Figure 5.38. Although the parameters deviate from fiber B-DNA, even for the unmodified oligo, they do not clearly fall into a conformation other than B-DNA. A notable feature of Figure 5.38a is the fact that those residues with larger negative slide and positive rise correspond to modified guanines. This is also observed in Figure 5.30. Figure 5.38b shows that although the inclination of the base pairs for all modified B-DNA's is more negative than that for the unmodified B-DNA, it does not fall out of the range that is feasible for B-DNA's in general. Correlations between roll and twist are shown in Figure 5.38c and fall over a broad range of values. All of the modified B-DNA's are underwound relative to both A-DNA and B-DNA. Other than C3/G4, the unmodified DNA has twist values that give reasonable agreement with the fiber structures. The roll values for the modified B-DNA's fall into a range intermediate between A and B-DNA. Variations in twist and roll are often coupled to changes in one or more translational parameters and are often sequence dependent. ${ }^{151}$ 
a)
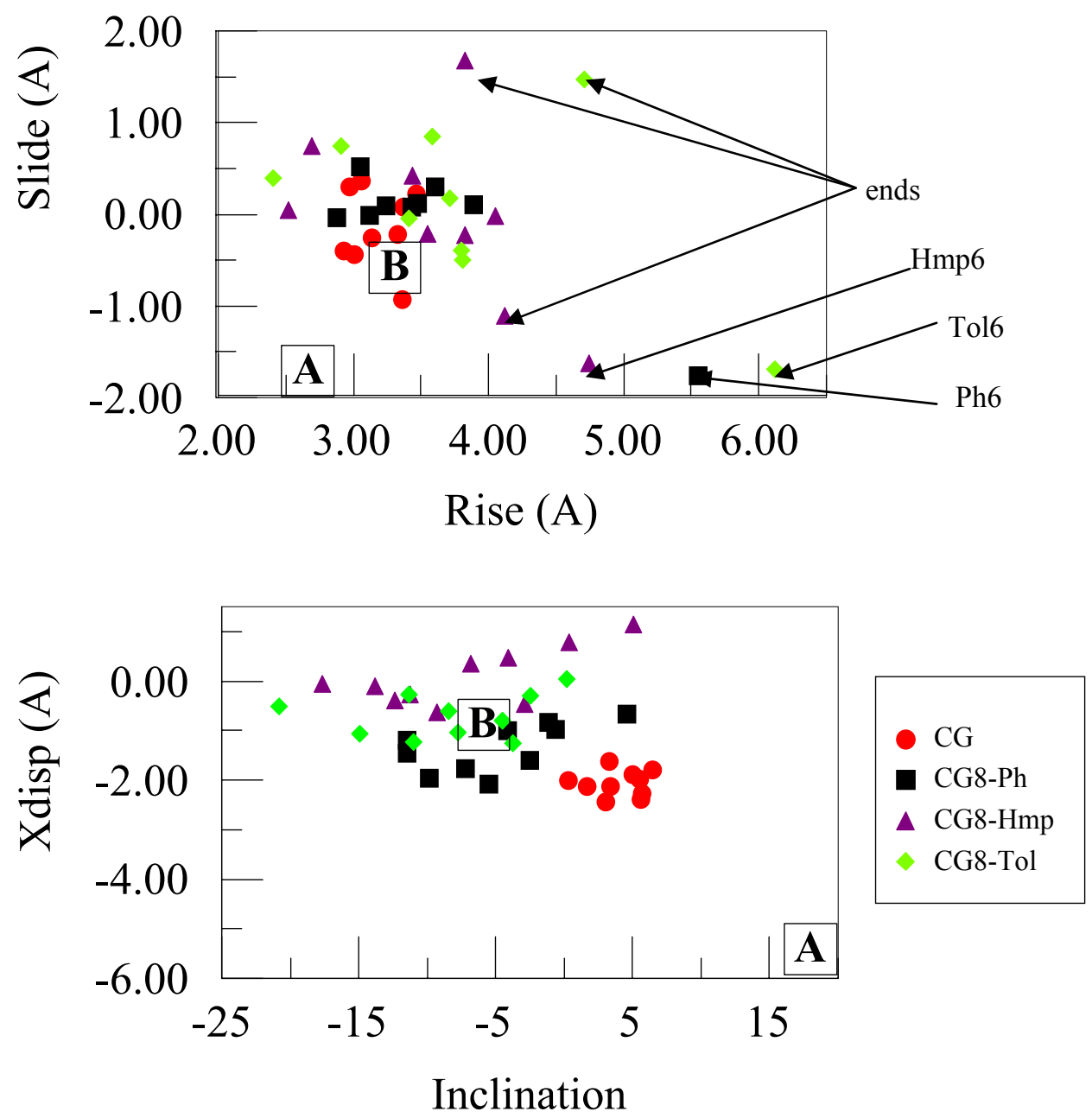

c)

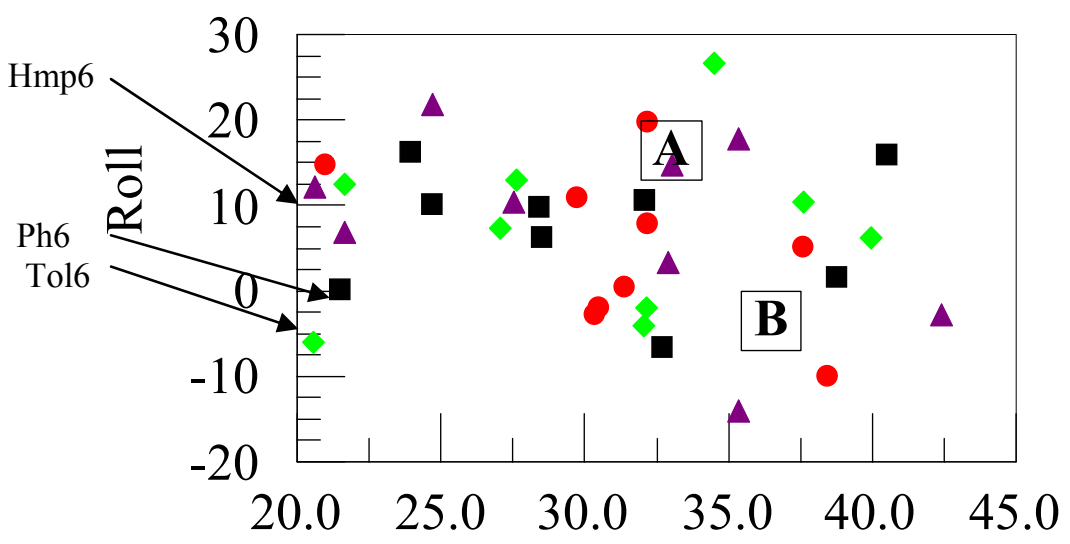

Twist

Figure 5.38. Helical parameters calculated by CURVES for the unmodified and modified BDNA's. Parameters for A-DNA and B-DNA fibers are indicated by the boxed A's and B's, respectively. ${ }^{150}$ Inclination, roll, and twist are in units of degrees. 


\subsubsection{Energy Analysis}

Figure 5.39 shows a comparison of the total energy over the course of the production runs for the unmodified, as well as the phenyl and tolyl-modified DNA's in the B and Z conformations. The order from highest to lowest energy, when comparing all of the production runs was $\mathbf{C G}(\mathrm{Z}), \mathbf{C G}, \mathbf{C G}^{8-\mathrm{Ph}}, \mathbf{C G}^{8-\mathrm{Ph}}(\mathrm{Z}), \mathbf{C G}^{8-\mathrm{Tol}}, \mathbf{C G}^{8-\mathrm{Hmp}}, \mathbf{C G}^{8-\mathrm{Tol}}(\mathrm{Z})$, and $\mathbf{C G}^{8-\mathrm{Hmp}}(\mathrm{Z})$. At first glance, it seems that C8-aryl modification of G6 and G16 stabilize the Z-DNA's over the B form. Examination of the modified DNA's suggests an explanation for this result, since in the BDNA conformation, the modifications are in a sterically unfavorable position relative to their position in the Z-DNA conformation. Upon closer inspection, a linear correlation between the system size, i.e., the number of solvent molecules, and total energy is apparent. Figure 5.40 illustrates this relationship.

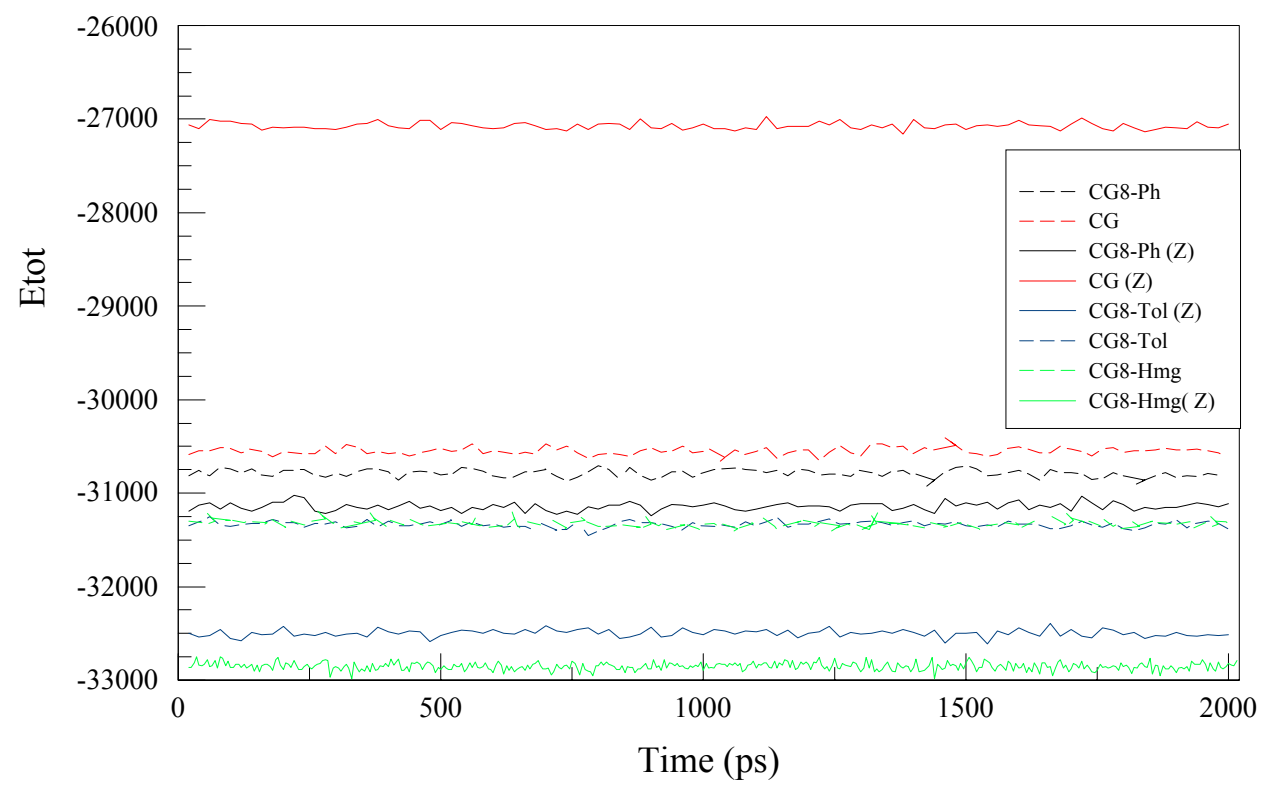

Figure 5.39. Total (Etot) energy comparison for all production runs. Energy is in units of $\mathrm{kcal} /$ mole. 


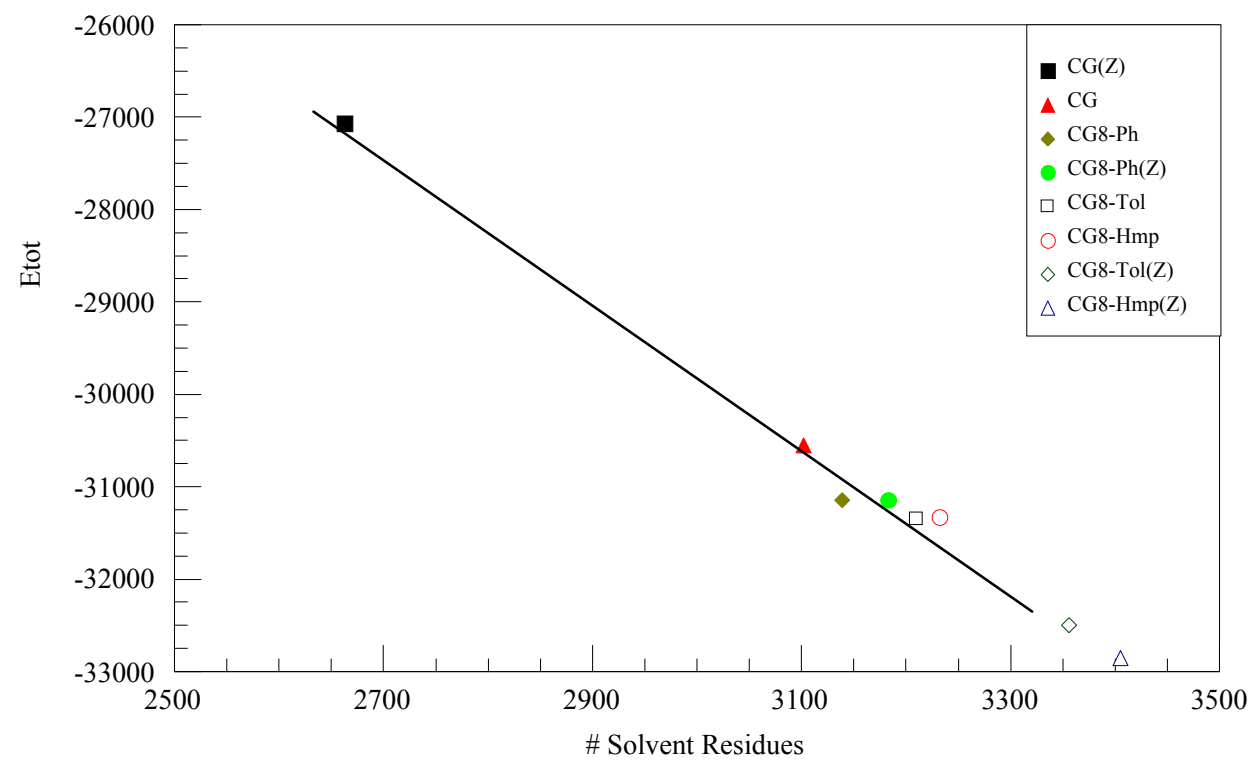

Figure 5.40. Linear relationship between the number of solvent residues and average total energy calculated during the production MD simulation.

To further explore the energetic aspects of the simulations, the MM-PBSA module of Amber 7 was employed. MM-PBSA is a method to estimate the free energy of a system; it can be used to evaluate free energies of binding or to calculate the absolute free energies of molecules in solution. In this case, our goal was to compare solvation free energies of the various systems studied. The production simulation trajectory was post-processed to remove solvent and counterions. Estimations of the electrostatic component of the solvation free energies for the nucleic acid solute were performed using the generalized Born (GB) method in Amber $^{152}$ and alternatively, the Poisson-Boltzman (PB) method in Delphi ${ }^{125}$ (Tables 5.14. and 5.15). The following equations define the terms used in the free energy calculations:

$$
\begin{aligned}
& \triangle G_{-} G B=G B T O T-T S T O T \\
& G B T O T=G B S O L+G A S \\
& G A S=I N T+V D W+E L E \\
& G B S O L=G B S U R F+G B \\
& G B E L E=G B+E L E
\end{aligned}
$$




$$
\begin{aligned}
& \text { TSTOT }=\text { TSTRA }+ \text { TSVIB }+ \text { TSROT } \\
& \triangle G \_P B=P B T O T-T S T O T \\
& P B T O T=P B S O L+G A S \\
& P B S O L=P B S U R F+P B \\
& P B E L E=P B+E L E
\end{aligned}
$$

The free energy $\left(\Delta G_{-} G B\right.$ or $\left.\triangle G_{-} P B\right)$ reflects the sum of the average molecular mechanical energy (GAS), solvation free energy (GBTOT or PBTOT), and the negative of solute entropy multiplied by temperature (TSTOT). The average molecular mechanical energy is the sum of several components computed in vacuo: the internal (bond, angle, and torsion) energy (INT), the non-bonded Van der Waals (VDW), and electrostatic (ELE) energies. GBSUR/PBSUR refers to the non-polar contribution to solvation free energy, $G B / P B C A L$ refers to the polar contribution to solvation free energy, and $G B S O L / P B S O L$ is the sum of $G B / P B C A L$ and GBSUR/PBSUR. GBELE/PBELE is the sum of GB/PBCAL and ELE, and GBTOT/PBTOT is the sum of GBSOL/PBSOL and GAS. The entropies were calculated during the MM-PBSA analysis using the nmode program in Amber. TSROT, TSVIB, and TSTRA are the rotational, vibrational, and translational components of entropy respectively, multiplied by $300 \mathrm{~K}$, and TSTOT is the sum of the three components. $\triangle \triangle G_{-} B Z(Z B)$ is the difference between $\triangle G_{-} P B$ and $\triangle P B \_B Z(Z B)$ is the difference between PBTOT in the B-DNA and Z-DNA conformations for each set of oligos.

Both Poisson-Boltzmann and Generalized Born models were used to evaluate the electrostatic component of the solvation free energy. The PB approach is well established and used widely in the literature. It has gained acceptance as a convenient and reliable alternative to explicit solvent in energy calculations. The GB methodology is still under development but it seemed worthwhile to utilize it for comparison's sake since it saves computational time and is 
incorporated into the Amber set of programs. The GB approach has been found useful for small molecules but may need further evaluation for macromolecular applications. ${ }^{153}$

For the Poisson-Boltman approach using Delphi, a grid spacing of $0.25 \AA$ with a cubic lattice, whose linear dimensions were about 50\% larger than the longest dimension of the solute, was used. Potentials at the boundaries of the finite-difference lattice were set to sum of the Debye-Huckel potentials. ${ }^{126}$ The linear solution to the Poisson Boltmann equation was used and salt effects were not included implicitly in the continuum model. The dielectric boundary is taken as the molecular surface defined by a $1.4 \AA$ probe sphere and by spheres centered on each atom with radii taken from the PARSE parameter $\operatorname{set}^{131}(\mathrm{H}=1.0, \mathrm{C}=1.7, \mathrm{~N}=1.5, \mathrm{O}=1.4$, and $\mathrm{P}=2.0$ ). Partial charges on the solute atoms are taken from the Cornell force field, ${ }^{154}$ in order to be consistent with the energetics of the explicit simulations. The solute is assigned a dielectric of one, and the solvent dielectric is set to 80 .

The Generalized Born approach used the standard pairwise generalized Born model with parameters described by Tsui and Case. ${ }^{129}$ The hydrogen atoms are treated as three different groups based on their covalently bonded partners: the hydroxyl hydrogens, the amino hydrogens, and the aliphatic hydrogens with radii of $0.8,1.2$, and $1.3 \AA$, respectively. The order of size correlates with the electronegativity of their bonding partners and matches the order of hydrogen sizes used in the Cornell force field for the gas-phase portion of the energy function (i.e. $\mathrm{HC}>\mathrm{HN}>\mathrm{HO}$ ). ${ }^{152}$ Atomic radii values for $\mathrm{C}, \mathrm{O}, \mathrm{N}$, and $\mathrm{P}$ in the $\mathrm{GB}$ calculations are 1.7, $1.5,1.55$, and $1.85 \AA$, respectively. The GB simulations in this study were performed with a solvent dielectric of 80 and a solute dielectric of unity.

Entropy contribution to free energy was estimated using a harmonic approximation to the normal modes found in the nmode module of Amber; this requires minimization of each frame 
included in the calculation (in this case every $10^{\text {th }}$ frame from 500 to 1950 ). A distance dielectric constant of 4 was used, and 100 steps of steepest descent minimization was followed by conjugate gradient minimization up to 5,000,000 steps until the RMSD of the Cartesian elements of the gradient were less than $10^{-4} \mathrm{kcal} / \mathrm{mol}-\AA$.

Using the linear PB method for the calculation of free energy the order from highest to lowest energy, when comparing all of the production runs, was $\mathbf{C G}^{8-\mathrm{Ph}}(\mathbf{B}), \mathbf{C G}^{\mathbf{8 - H m p}}(\mathbf{Z}), \mathbf{C G}^{8-}$ ${ }^{\mathrm{Hmp}}(\mathbf{B}), \mathbf{C G}^{8-\mathrm{Ph}}(\mathrm{Z}), \mathbf{C G}^{8-\mathrm{Tol}}(\mathrm{B}), \mathbf{C G}^{8-\mathrm{Tol}}(\mathrm{Z}), \mathbf{C G}(\mathrm{Z})$, and $\mathbf{C G}(\mathbf{B})$. The results from the same calculation using the GB method were $\mathbf{C G}^{\text {8-Ph }}(\mathbf{B}), \mathbf{C G}^{8-\mathrm{Hmp}}(\mathbf{Z}), \mathbf{C G}^{8-\mathrm{Hmp}}(\mathbf{B}), \mathbf{C G}^{8-\mathrm{Tol}}(\mathbf{B}), \mathbf{C G}^{8-}$ ${ }^{\text {Tol }}(\mathbf{Z}), \mathbf{C G}(\mathbf{Z}), \mathbf{C G}(\mathbf{B})$, and $\mathbf{C G}^{\text {8-Ph }}(\mathbf{Z})$. The relative order of free and total energies for the structures are outlined in Table 5.13 below; these comparisons serve to highlight the differences, or lack thereof, in the overall order obtained from the PB method versus the GB method. True energy comparisons can only be made from a structure in the B conformation to its corresponding structure in the $\mathrm{Z}$ conformation (i.e. apples to apples and phenyls to phenyls). Although the individual results varied, both approaches to the calculations of free energy led to the same overall conclusions. The $\mathrm{Z}$ form of DNA was favored over the $\mathrm{B}$ for the phenyl and tolyl modifications. The unmodified, and to a lesser extent, the hydroxymethylphenyl-modified DNA favored the B conformation. Table 5.13 also includes the total energies calculated during the production run. The total energies are inversely proportion to the number of solvent molecules, that is, $\mathbf{C G}(\mathbf{Z})$ has the least amount of water molecules and the highest energy; $\mathbf{C G}^{\mathbf{8 -}}$ Hmp $(\mathbf{Z})$ has the most waters and lowest total energy. For this reason, one cannot make any correlations between total and solvation free energies. 
Table 5.13. Relative order of total and free energies for the modified and unmodified DNA's in the $\mathrm{B}$ and $\mathrm{Z}$ conformations from the total energy calculated during the MD simulation, and the $\mathrm{PB}$ and GB solvent models. The structures are listed in order from highest to lowest energy.

\begin{tabular}{|l|l|l|}
\hline Total & PB & GB \\
\hline $\mathrm{CG}(\mathrm{Z})$ & $\mathrm{CG}^{8-\mathrm{Ph}}(\mathrm{B})$ & $\mathrm{CG}^{8-\mathrm{Ph}}(\mathrm{B})$ \\
\hline $\mathrm{CG}(\mathrm{B})$ & $\mathrm{CG}^{8-\mathrm{Hmp}}(\mathrm{Z})$ & $\mathrm{CG}^{8-\mathrm{Hmp}}(\mathrm{Z})$ \\
\hline $\mathrm{CG}^{8-\mathrm{Ph}}(\mathrm{B})$ & $\mathrm{CG}^{8-\mathrm{Hmp}}(\mathrm{B})$ & $\mathrm{CG}^{8-\mathrm{Hmp}}(\mathrm{B})$ \\
\hline $\mathrm{CG}^{8-\mathrm{Ph}}(\mathrm{Z})$ & $\mathrm{CG}^{8-\mathrm{Ph}}(\mathrm{Z})$ & $\mathrm{CG}^{8-\mathrm{Tol}}(\mathrm{B})$ \\
\hline $\mathrm{CG}^{8-\mathrm{Tol}}(\mathrm{B})$ & $\mathrm{CG}^{8-\mathrm{Tol}}(\mathrm{B})$ & $\mathrm{CG}^{8-\mathrm{Tol}}(\mathrm{Z})$ \\
\hline $\mathrm{CG}^{8-\mathrm{Hmp}}(\mathrm{B})$ & $\mathrm{CG}^{8-\mathrm{Tol}}(\mathrm{Z})$ & $\mathrm{CG}(\mathrm{Z})$ \\
\hline $\mathrm{CG}^{8-\mathrm{Tol}}(\mathrm{Z})$ & $\mathrm{CG}(\mathrm{Z})$ & $\mathrm{CG}(\mathrm{B})$ \\
\hline $\mathrm{CG}^{8-\mathrm{Hmp}}(\mathrm{Z})$ & $\mathrm{CG}(\mathrm{B})$ & $\mathrm{CG}^{8-\mathrm{Ph}}(\mathrm{Z})$ \\
\hline
\end{tabular}

Tables 5.14 and 5.15 show a breakdown of the solvation free energies into individual components. The polar contribution to free energy (PBCAL) favors the Z-DNA's; this is due to the more effective solvation of the phosphate groups in Z-DNA relative to B-DNA because the charges are closer together. On the other hand, the molecular mechanical energy favors the BDNA's predominately due to the non-bonded electrostatic energy term (ELE), also caused by the smaller separation between the phosphate anions of opposite strands in Z-DNA relative to its B counterpart. ${ }^{79} \mathbf{C G}^{\mathbf{8}-\mathbf{T o l}}$ has a higher non-bonded electrostatic energy and a lower solvation free energy relative to all of the other B-DNA's; this may be caused by the relatively wider major groove and narrower minor groove which could impact phosphate-phosphate distances and effective solvation. The tolyl-modified DNA in the $\mathrm{Z}$ form also has a higher non-bonded electrostatic term and lower solvation free energy term relative to the other Z-DNA's, but no such correlation with groove widths can be made. The unmodified B form is more stable relative to its $\mathrm{Z}$ counterpart by $0.80 \mathrm{kcal} / \mathrm{mol}$. Removing the entropy contribution, the unmodified $\mathrm{Z}$ is favored by $10.74 \mathrm{kcal} / \mathrm{mol}$. Conformational entropy is an important factor in the nonelectostatic forces stabilizing the B-DNA conformation. ${ }^{124}$ 
To examine the effect of salt concentration on the PB results additional calculations were performed in $0.2 \mathrm{M}$ and $2 \mathrm{M} \mathrm{NaCl}$ using the non-linear solution to the Poisson-Boltzman equation. The addition of salt had little effect on the free energies of the B-DNAs'. The ZDNAs'; however, increased in stability relative to the B's as salt concentration was increased. In fact, with the addition of just $0.2 \mathrm{M} \mathrm{NaCl}$ the relative stability of the unmodified and Hmp modified DNAs reversed, with the $\mathrm{Z}$ favored over the $\mathrm{B}$. 
Table 5.14. Average energies (in $\mathrm{kcal} / \mathrm{mol}$ and rounded to four significant digits) and standard errors of the mean energies (in parentheses) from 145 equally spaced configurations sampled from frames 500-1950 of the B-DNA trajectories calculated as discussed above. Numbers in parentheses represent the standard error of the mean, i.e., the mean divided by the square root of the number of snapshots sampled.

\begin{tabular}{|c|c|c|c|c|}
\hline ENERGY & CG (B) & $\mathrm{CG}^{8-\mathrm{Ph}}(\mathrm{B})$ & $\mathrm{CG}^{8-\mathrm{Tol}}(\mathrm{B})$ & $\mathrm{CG}^{8-\mathrm{Hmp}}(\mathrm{B})$ \\
\hline ELE & $\begin{array}{l}-817.4 \\
(2.9)\end{array}$ & $\begin{array}{l}-821.8 \\
(2.9)\end{array}$ & $\begin{array}{l}-698.3 \\
(3.6)\end{array}$ & $\begin{array}{l}-812.71 \\
(2.8)\end{array}$ \\
\hline VDW & $\begin{array}{l}-164.3 \\
(0.8)\end{array}$ & $\begin{array}{l}-169.6 \\
(0.8)\end{array}$ & $\begin{array}{l}-171.8 \\
(0.8)\end{array}$ & $\begin{array}{l}-171.1 \\
(0.81)\end{array}$ \\
\hline INT & $\begin{array}{l}939.7 \\
(1.6)\end{array}$ & $\begin{array}{l}968.4 \\
(1.6)\end{array}$ & $\begin{array}{l}975.2 \\
(1.68)\end{array}$ & $\begin{array}{l}955.9 \\
(1.8)\end{array}$ \\
\hline GAS & $\begin{array}{l}-41.96 \\
(3.1) \\
\end{array}$ & $\begin{array}{l}-23.00 \\
(3.1) \\
\end{array}$ & $\begin{array}{l}105.0 \\
(3.76)\end{array}$ & $\begin{array}{l}-27.9 \\
(0.0) \\
\end{array}$ \\
\hline GBSUR & $\begin{array}{l}22.20 \\
(0.0)\end{array}$ & $\begin{array}{l}22.56 \\
(0.0) \\
\end{array}$ & $\begin{array}{l}22.68 \\
(0.0)\end{array}$ & $\begin{array}{l}22.55 \\
(0.0)\end{array}$ \\
\hline GB & $\begin{array}{l}-4505 \\
(2.7)\end{array}$ & $\begin{array}{l}-4486 \\
(2.7)\end{array}$ & $\begin{array}{l}-4624 \\
(3.44) \\
\end{array}$ & $\begin{array}{l}-4487 \\
(3.4)\end{array}$ \\
\hline GBSOL & $\begin{array}{l}-4482 \\
(2.7) \\
\end{array}$ & $\begin{array}{l}-4464 \\
(2.7) \\
\end{array}$ & $\begin{array}{l}-4601 \\
(3.45) \\
\end{array}$ & $\begin{array}{l}-4464 \\
(3.4) \\
\end{array}$ \\
\hline GBELE & $\begin{array}{l}-5322 \\
(0.9) \\
\end{array}$ & $\begin{array}{l}-5308 \\
((0.9) \\
\end{array}$ & $\begin{array}{l}-5322 \\
(1.0) \\
\end{array}$ & $\begin{array}{l}-5300 \\
(1.0) \\
\end{array}$ \\
\hline GBTOT & $\begin{array}{l}-4524 \\
(1.5) \\
\end{array}$ & $\begin{array}{l}-4487 \\
(1.5) \\
\end{array}$ & $\begin{array}{l}-4496 \\
(1.6) \\
\end{array}$ & $\begin{array}{l}-4492 \\
(1.6) \\
\end{array}$ \\
\hline TSTRA & $\begin{array}{l}15.57 \\
(0.0)\end{array}$ & $\begin{array}{l}15.59 \\
(0.0)\end{array}$ & $\begin{array}{l}15.60 \\
(0.0)\end{array}$ & $\begin{array}{l}15.60 \\
(0.0)\end{array}$ \\
\hline TSROT & $\begin{array}{l}15.18 \\
(0.0) \\
\end{array}$ & $\begin{array}{l}15.22 \\
(0.0) \\
\end{array}$ & $\begin{array}{l}15.23 \\
(0.0) \\
\end{array}$ & $\begin{array}{l}15.21 \\
(0.0) \\
\end{array}$ \\
\hline TSVIB & $\begin{array}{l}490.3 \\
(0.2)\end{array}$ & $\begin{array}{l}497.3 \\
(0.2) \\
\end{array}$ & $\begin{array}{l}501.7 \\
(0.2)\end{array}$ & $\begin{array}{l}500.3 \\
(0.3)\end{array}$ \\
\hline TSTOT & $\begin{array}{l}521.0 \\
(0.2) \\
\end{array}$ & $\begin{array}{l}528.1 \\
(0.2) \\
\end{array}$ & $\begin{array}{l}532.5 \\
(0.2)\end{array}$ & $\begin{array}{l}531.1 \\
(0.3) \\
\end{array}$ \\
\hline PBSUR & $\begin{array}{l}17.63 \\
(0.0) \\
\end{array}$ & $\begin{array}{l}17.90 \\
(0.0) \\
\end{array}$ & $\begin{array}{l}17.99 \\
(0.0) \\
\end{array}$ & $\begin{array}{l}17.89 \\
(0.0) \\
\end{array}$ \\
\hline PBCAL & $\begin{array}{l}-4858 \\
(2.7) \\
\end{array}$ & $\begin{array}{l}-4839 \\
(2.7) \\
\end{array}$ & $\begin{array}{l}-4977 \\
(3.5) \\
\end{array}$ & $\begin{array}{l}-4838 \\
(2.6) \\
\end{array}$ \\
\hline PBSOL & $\begin{array}{l}-4841 \\
(2.7)\end{array}$ & $\begin{array}{l}-4821 \\
(2.7)\end{array}$ & $\begin{array}{l}-4959 \\
(3.5)\end{array}$ & $\begin{array}{l}-4820 \\
(2.6)\end{array}$ \\
\hline PBELE & $\begin{array}{l}-5676 \\
(0.9) \\
\end{array}$ & $\begin{array}{l}-5661 \\
(0.9) \\
\end{array}$ & $\begin{array}{l}-5676 \\
(1.0) \\
\end{array}$ & $\begin{array}{l}-5651 \\
(1.2) \\
\end{array}$ \\
\hline PBTOT & $\begin{array}{l}-4883 \\
(1.5) \\
\end{array}$ & $\begin{array}{l}-4844 \\
(1.5) \\
\end{array}$ & $\begin{array}{l}-4854 \\
(1.6) \\
\end{array}$ & $\begin{array}{l}-4848 \\
(1.7) \\
\end{array}$ \\
\hline$\Delta \mathbf{G} \_\mathbf{G B}$ & -5046 & -5015 & -5028 & -5024 \\
\hline$\Delta G \_P B$ & -5404 & -5372 & -5387 & -5379 \\
\hline$\Delta \Delta G \_B Z$ & -0.80 & 12.15 & 15.45 & -0.29 \\
\hline$\triangle \mathrm{PB} B \mathrm{BZ}$ & 10.74 & 16.17 & 17.76 & -3.03 \\
\hline
\end{tabular}


Table 5.15. Average energies (in $\mathrm{kcal} / \mathrm{mol}$ and rounded to four significant digits) and standard errors of the mean energies (in parentheses) from 145 equally spaced configurations sampled from frames 500-1950 of the Z-DNA trajectories calculated as discussed above. Numbers in parentheses represent the standard error of the mean, i.e., the mean divided by the square root of the number of snapshots sampled.

\begin{tabular}{|c|c|c|c|c|}
\hline ENERGY & CG (Z) & $\mathrm{CG}^{8-\mathrm{Ph}}(\mathrm{Z})$ & $\mathrm{CG}^{8-\mathrm{Tol}}(\mathrm{Z})$ & $\mathrm{CG}^{8-\mathrm{Hmp}}(\mathrm{Z})$ \\
\hline ELE & $\begin{array}{l}-63.41 \\
(5.7)\end{array}$ & $\begin{array}{l}-65.69 \\
(5.3)\end{array}$ & $\begin{array}{l}53.67 \\
(6.78)\end{array}$ & $\begin{array}{l}-24.78 \\
(5.97)\end{array}$ \\
\hline VDW & $\begin{array}{l}-196.2 \\
(0.7)\end{array}$ & $\begin{array}{l}-194.2 \\
(0.8)\end{array}$ & $\begin{array}{l}-195.2 \\
(0.8)\end{array}$ & $\begin{array}{l}-196.5 \\
(0.67)\end{array}$ \\
\hline INT & $\begin{array}{l}953.5 \\
(1.6)\end{array}$ & $\begin{array}{l}975.8 \\
(1.5) \\
\end{array}$ & $\begin{array}{l}981.0 \\
(1.5) \\
\end{array}$ & $\begin{array}{l}983.5 \\
(1.59)\end{array}$ \\
\hline GAS & $\begin{array}{l}693.9 \\
(5.6)\end{array}$ & $\begin{array}{l}715.8 \\
(5.1)\end{array}$ & $\begin{array}{l}839.4 \\
(6.5)\end{array}$ & $\begin{array}{l}762.3 \\
(5.95)\end{array}$ \\
\hline GBSUR & $\begin{array}{l}20.61 \\
(0.0)\end{array}$ & $\begin{array}{l}21.55 \\
(0.0)\end{array}$ & $\begin{array}{l}21.83 \\
(0.0)\end{array}$ & $\begin{array}{l}21.94 \\
(0.0)\end{array}$ \\
\hline GB & $\begin{array}{l}-5243 \\
(5.4)\end{array}$ & $\begin{array}{l}-5281 \\
(5.1)\end{array}$ & $\begin{array}{l}-5367 \\
(6.4)\end{array}$ & $\begin{array}{l}-5267 \\
(5.6\end{array}$ \\
\hline GBSOL & $\begin{array}{l}-5222 \\
(5.4)\end{array}$ & $\begin{array}{l}-5260 \\
(5.1)\end{array}$ & $\begin{array}{l}-5346 \\
(6.4)\end{array}$ & $\begin{array}{l}-5245 \\
(5.6)\end{array}$ \\
\hline GBELE & $\begin{array}{l}-5306 \\
(0.9)\end{array}$ & $\begin{array}{l}-5347 \\
(0.8)\end{array}$ & $\begin{array}{l}-5314 \\
(1.0)\end{array}$ & $\begin{array}{l}-5292 \\
(1.0)\end{array}$ \\
\hline GBTOT & $\begin{array}{l}-4528 \\
(1.4) \\
\end{array}$ & $\begin{array}{l}-4544 \\
(1.5)\end{array}$ & $\begin{array}{l}-4506 \\
(1.5)\end{array}$ & $\begin{array}{c}-4483 . \\
(1.6)\end{array}$ \\
\hline TSTRA & $\begin{array}{l}15.57 \\
(0.0)\end{array}$ & $\begin{array}{l}15.59 \\
(0.0)\end{array}$ & $\begin{array}{l}15.60 \\
(0.0)\end{array}$ & $\begin{array}{l}15.60 \\
(0.0)\end{array}$ \\
\hline TSROT & $\begin{array}{l}15.20 \\
(0.0) \\
\end{array}$ & $\begin{array}{l}15.23 \\
(0.0) \\
\end{array}$ & $\begin{array}{l}15.24 \\
(0.0) \\
\end{array}$ & $\begin{array}{l}15.25 \\
(0.0) \\
\end{array}$ \\
\hline TSVIB & $\begin{array}{l}478.7 \\
(0.2)\end{array}$ & $\begin{array}{l}493.3 \\
(0.2)\end{array}$ & $\begin{array}{l}499.3 \\
(0.2)\end{array}$ & $\begin{array}{l}503.0 \\
(01)\end{array}$ \\
\hline TSTOT & $\begin{array}{l}509.5 \\
(0.2)\end{array}$ & $\begin{array}{l}524.1 \\
(0.2)\end{array}$ & $\begin{array}{l}530.2 \\
(0.2)\end{array}$ & $\begin{array}{l}533.9 \\
(0.1)\end{array}$ \\
\hline PBSUR & $\begin{array}{l}16.43 \\
(0.0) \\
\end{array}$ & $\begin{array}{l}17.14 \\
(0.0)\end{array}$ & $\begin{array}{l}17.35 \\
(0.0)\end{array}$ & $\begin{array}{l}17.43 \\
(0.0)\end{array}$ \\
\hline PBCAL & $\begin{array}{l}-5604 \\
(5.5)\end{array}$ & $\begin{array}{l}-5593 \\
(5.0)\end{array}$ & $\begin{array}{l}-5729 \\
(6.5)\end{array}$ & $\begin{array}{l}-5625 \\
(5.6)\end{array}$ \\
\hline PBSOL & $\begin{array}{l}-5587 \\
(5.5)\end{array}$ & $\begin{array}{l}-5576 \\
(5.0)\end{array}$ & $\begin{array}{l}-5712 \\
(6.5)\end{array}$ & $\begin{array}{l}-5607 \\
(5.6)\end{array}$ \\
\hline PBELE & $\begin{array}{l}-5667 \\
(1.0)\end{array}$ & $\begin{array}{l}-5659 \\
(0.9)\end{array}$ & $\begin{array}{l}-5675 \\
(1.0)\end{array}$ & $\begin{array}{l}-5649 \\
(1.0)\end{array}$ \\
\hline PBTOT & $\begin{array}{l}-4893 \\
(1.4)\end{array}$ & $\begin{array}{l}-4860 \\
(1.5) \\
\end{array}$ & $\begin{array}{l}-4872 \\
(0.5)\end{array}$ & $\begin{array}{l}-4845 \\
(1.6)\end{array}$ \\
\hline$\Delta \mathbf{G}$ _GB & -5038 & -5068 & -5036 & -5016 \\
\hline$\Delta G \_P B$ & -5403 & -5384 & -5402 & -5379 \\
\hline$\Delta \Delta G \_Z B$ & 0.80 & -12.15 & -15.45 & 0.29 \\
\hline$\triangle P B \_Z B$ & -10.74 & -16.17 & -17.76 & 3.03 \\
\hline
\end{tabular}


Table 5.16. Average energies (in $\mathrm{kcal} / \mathrm{mol}$ and rounded to four significant digits) from 145 equally spaced configurations sampled from frames 500-1950 of the B and Z-DNA trajectories calculated as discussed above varying salt concentration for the PB calculations.

\begin{tabular}{|c|c|c|c|c|c|c|c|c|c|c|c|c|}
\hline \multirow[b]{2}{*}{ ENERGY } & \multicolumn{4}{|c|}{ 0 M Salt } & \multicolumn{4}{|c|}{ 0.2 M Salt } & \multicolumn{4}{|c|}{ 2M Salt } \\
\hline & CG (B) & $\mathrm{CG}^{8-\mathrm{Ph}}(\mathrm{B})$ & $\mathrm{CG}^{8-\mathrm{Tol}}(\mathrm{B})$ & $C G^{\mathrm{Hmp}}(\mathrm{B})$ & CG (B) & $\mathrm{CG}^{8-\mathrm{Ph}}(\mathrm{B})$ & $\mathrm{CG}^{8-\mathrm{Tol}}(\mathrm{B})$ & $C G^{8 \mathrm{Hmp}}(\mathrm{B})$ & CG (B) & $\mathrm{CG}^{8-\mathrm{Ph}}(\mathrm{B})$ & $\mathrm{CG}^{8 \mathrm{Tol}}(\mathrm{B})$ & $\mathrm{CG}^{8 \mathrm{Hmp}}(\mathrm{B})$ \\
\hline $\mathbf{G A S}^{\mathbf{a}}$ & -41.96 & -23.00 & 105.0 & -27.94 & -41.96 & -23.00 & 105.0 & -27.94 & -41.96 & -23.00 & 105.0 & -27.94 \\
\hline TSTOT $^{\mathbf{a}}$ & 521.0 & 528.1 & 532.5 & 531.1 & 521.01 & 528.1 & 532.5 & 531.1 & 521.0 & 528.1 & 532.5 & 531.1 \\
\hline PBSUR & 17.6 & 17.90 & 17.99 & 17.89 & 17.63 & 17.90 & 17.99 & 17.89 & 17.63 & 17.90 & 17.99 & 17.89 \\
\hline PBCAL & -4858 & -4839 & -4977 & -4838 & -4859 & -4840 & -4978 & -4839 & -4859 & -4840 & -4978 & -4839 \\
\hline PBSOL & -4841 & -4821 & -4959 & -4820 & -4841 & -4822 & -4960 & -4820 & -4841 & -4822 & -4960 & -4821 \\
\hline PBELE & -5676 & -5661 & -5676 & -5651 & -5676 & -5661 & -5676 & -5651 & -5676 & -5662 & -5677 & -5651 \\
\hline PBTOT & -4883 & -4844 & -4854 & -4848 & -4883 & -4845 & -4855 & -4848 & -4883 & -4845 & -4855 & -4849 \\
\hline$\Delta G_{-} P B$ & -5404 & -5372 & -5387 & -5379 & -5404 & -5373 & -5388 & -5379 & -5404 & -5373 & -5388 & -5380 \\
\hline$\Delta \Delta G_{-} B Z$ & -0.8 & 12.15 & 15.45 & -0.29 & 4.95 & 16.13 & 19.47 & 3.94 & 6.16 & 17.27 & 20.57 & 5.16 \\
\hline ENERGY & CG (Z) & $C G^{8-P h}(Z)$ & $\mathrm{CG}^{8-\mathrm{Tol}}(\mathrm{Z})$ & $\mathrm{CG}^{8-\mathrm{Hmp}}(\mathrm{Z})$ & CG (Z) & $\mathrm{CG}^{8-\mathrm{Ph}}(\mathrm{Z})$ & $\mathrm{CG}^{8-\mathrm{Tol}}(\mathrm{Z})$ & $\mathrm{CG}^{8 \mathrm{Hmp}}(\mathrm{Z})$ & CG (Z) & $C G^{8-P h}(Z)$ & $C G^{8-T o l}(Z)$ & $\mathrm{CG}^{8 \mathrm{Hmp}}(\mathrm{Z})$ \\
\hline GAS & 693.9 & 715.8 & 839.4 & 762.3 & 693.9 & 715.8 & 839.4 & 762.3 & 693.9 & 715.8 & 839.4 & 762.3 \\
\hline TSTOT & 509.5 & 524.1 & 530.2 & 533.9 & 509.5 & 524.1 & 530.2 & 533.9 & 509.5 & 524.1 & 530.2 & 533.9 \\
\hline PBSUR & 16.43 & 17.14 & 17.35 & 17.43 & 16.43 & 17.14 & 17.35 & 17.43 & 16.43 & 17.14 & 17.35 & 17.43 \\
\hline PBCAL & -5604 & -5593 & -5729 & -5625 & -5608 & -5598 & -5734 & -5629 & -5610 & -5599 & -5735 & -5631 \\
\hline PBSOL & -5587 & -5576 & -5712 & -5607 & -5593 & -5581 & -5716 & -5611 & -5595 & -5582 & -5718 & -5613 \\
\hline PBELE & -5667 & -5659 & -5675 & -5649 & -5672 & -5663 & -5680 & -5654 & -5673 & -5665 & -5681 & -5656 \\
\hline PBTOT & -4893 & -4860 & -4872 & -4845 & -4900 & -4865 & -4877 & -4850 & -4901 & -4866 & -4878 & -4851 \\
\hline$\Delta G_{-} P B$ & -5403 & -5384 & -5402 & -5379 & -5409 & -5389 & -5407 & -5383 & -5411 & -5390 & -5408 & -5385 \\
\hline$\Delta \Delta G \_B Z$ & 0.8 & -12.15 & -15.45 & 0.29 & -4.95 & -16.13 & -19.47 & -3.94 & -6.16 & -17.27 & -20.57 & -5.16 \\
\hline
\end{tabular}

${ }^{a}$ For individual contributions to the gas phase and entropy calculations see Tables 5.15 and 5.16. Standard errors of the mean (data not shown) were the same as reported in Tables 5.14 and 5.15 . 


\subsection{Discussion}

Many structural parameters must be considered when comparing the unmodified and modified DNA's, as well as when comparing the respective conformations to canonical values. Electrostatic interactions between partial atomic charges are most important for the highly polarized CG base pairs. ${ }^{58}$ These interactions drive the strong preference for positive slide and negative slide in CG and GC steps, respectively. The GC step in Z-DNA is characterized by a twist of $-50.6^{\circ}$ and slide of $-1.1 \AA$. In contrast, for CG steps, the twist is $-9^{\circ}$ and the slide is $5.4 \AA$. Although these preferences are at the extreme of the slide axis and incompatible with the standard right-handed helix found in B-DNA, they are easily accommodated by the left-handed helix found in Z-DNA. Generally, electrostatic effects cause non-zero slide as found in A and ZDNA, and hydrophobic effects lead to zero-slide as found in B-DNA. ${ }^{58}$ As slide moves away from 0 , the contribution of hydrophobic effects to base step stability decreases $0.6 \mathrm{kcal} / \mathrm{mol}$ per angstrom from an estimated $2.87 \mathrm{kcal} / \mathrm{mol}^{58}$ When the conditions (high salt) are such that the unfavorable charge-charge interactions are screened, the thermodynamic force that drives conformational preferences for poly CG sequences shifts from minimization of surface area exposed to solvent to maximization of favorable stacking interactions.

The aryl-modified guanines in the B-DNA conformation exhibited negative slide, positive rise, negative inclination, and negative propeller twist relative to the unmodified DNA. The groove parameters were significantly affected by the modifications as well. The tolyl modification caused the most dramatic shifts from the unmodified structure, and the hydroxymethylphenyl caused the smallest deviations. Groove width, twist, inclination, and tilt are dependent on the same factors, the overall backbone path and orientation of the major axes of base pairs, ${ }^{155}$ so it follows that significant changes in inclination, for example, are accompanied 
by a large degree of change in groove width. The tolyl and to a lesser extent phenyl-modified oligos exhibited significant stretching and bending on the major grove side of the helix. As rise increases, resulting in stretching, propeller twist, roll, and tilt become significantly less restricted spatially because there are fewer constraints imposed on these variables at base steps. ${ }^{156}$ With less steric restrictions, the orientation variables are affected primarily by the overall electric field and base-backbone interactions; ${ }^{156}$ therefore, electrostatic interactions become more important than Van der Waals and the bases can shift away from conventional arrangements. These types of movements are akin to those exhibited during the binding of DNA to proteins and during the transition from one DNA conformation to another, implying that the conformational affects of the aryl guanine modifications have potential biological implications.

Figures 5.41 through 5.44 show close-up views of the unmodified, pheny, tolyl, and hydroxymethylphenyl -modified B-DNA's respectively, using a spacefill display generated in Protein Explorer. ${ }^{157}$ These figures serve to illustrate the conformational differences brought on by the addition of the aryl functional groups to $\mathrm{C} 8$ of guanine. Most notable for the phenyl and tolyl-modified DNA's is the increased rise between the $5^{\text {th }}, 6^{\text {th }}$, and $7^{\text {th }}$ base pair steps, the extended distance in the pathway between the phosphates of the $5^{\prime}$ strand and the $3^{\prime}$ strand on the major groove side, and the non-zero slide and unwinding illustrated by the positioning of, from top to bottom, G4-O6, G*6-O6, and G*16-O6. 


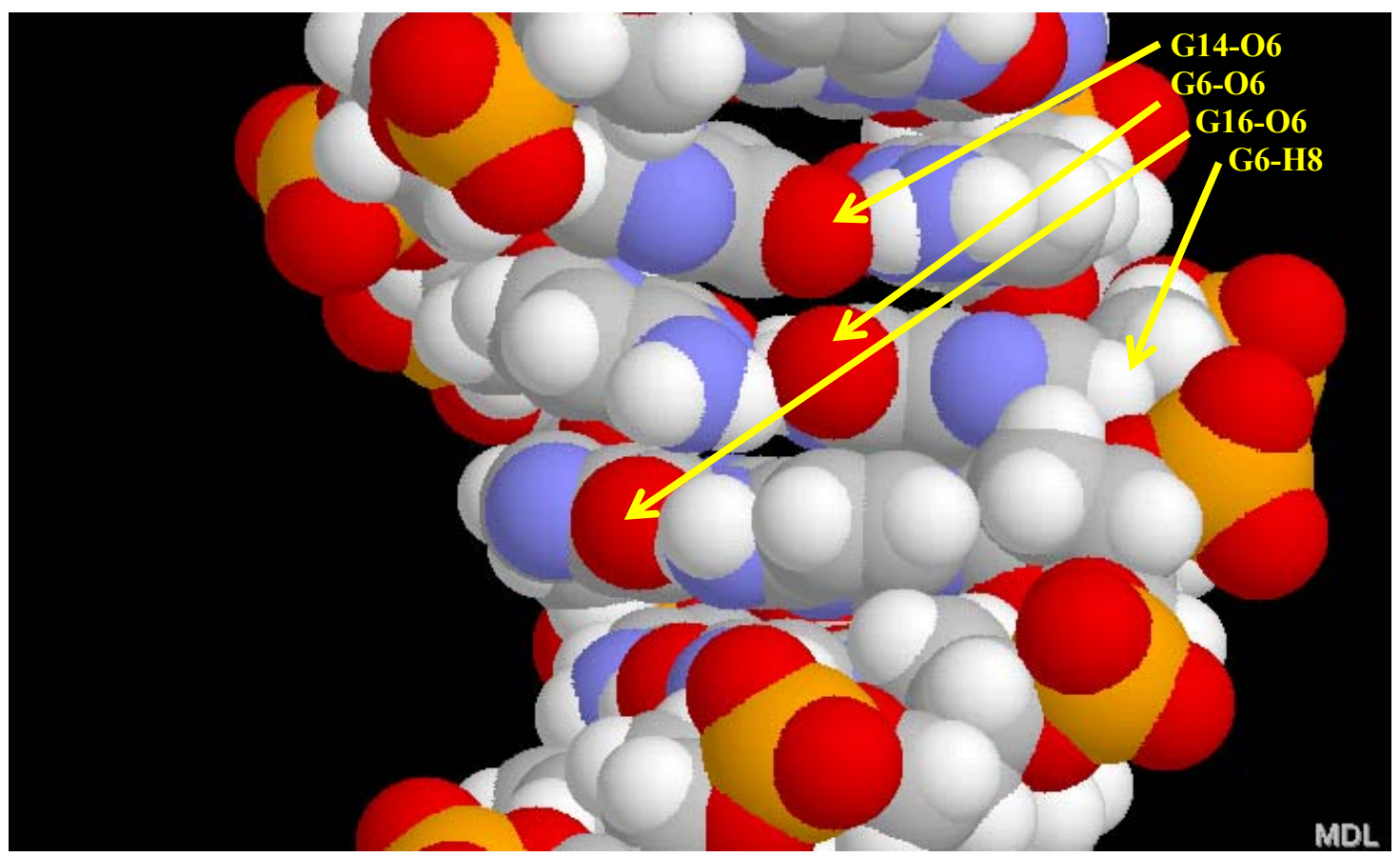

Figure 5.41. Close-up of, from bottom to top, the fourth through eighth base pair steps of the unmodified B-DNA

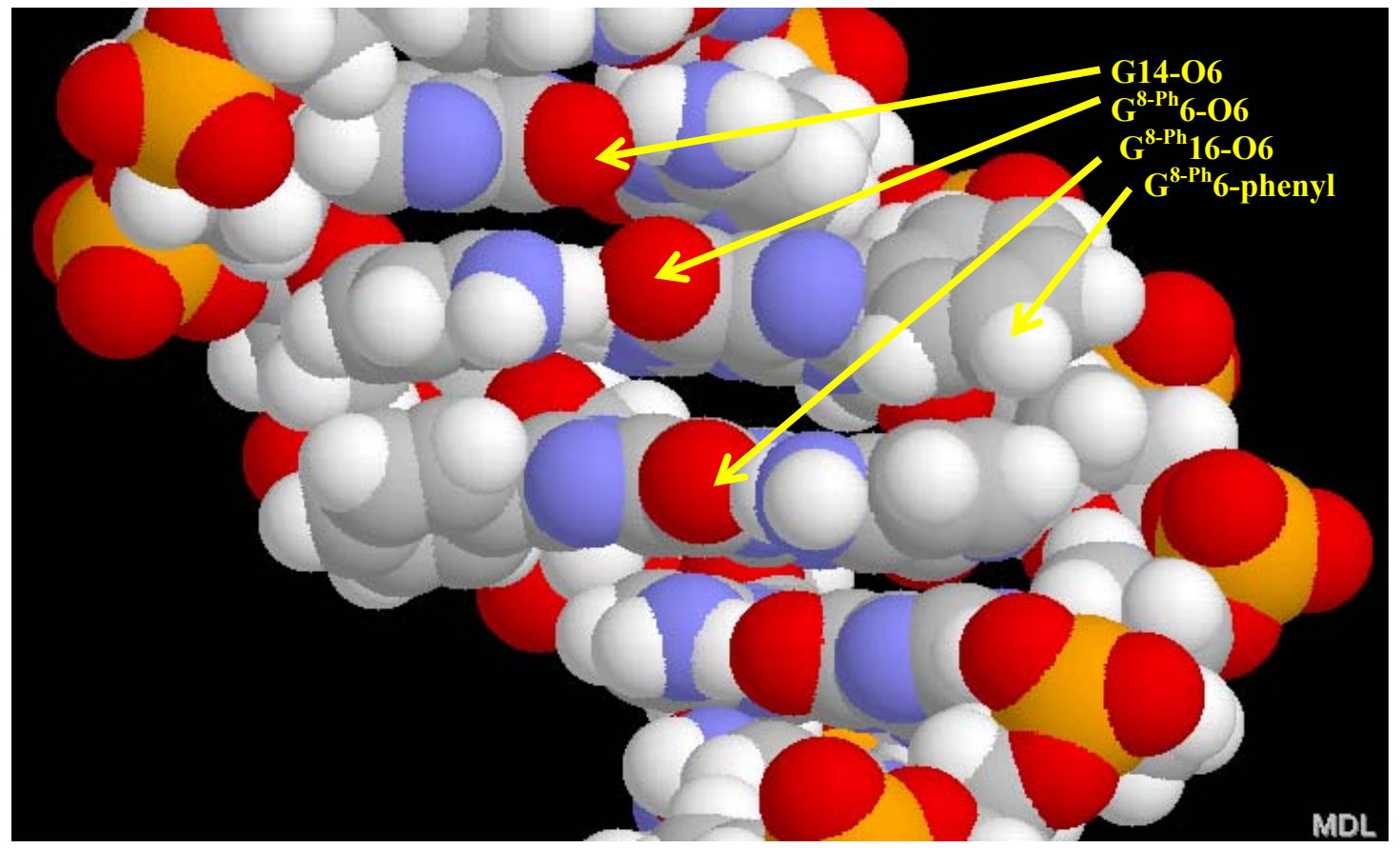

Figure 5.42. Close-up of, from bottom to top, the fourth through eighth base pair steps of the phenyl-modified B-DNA. 


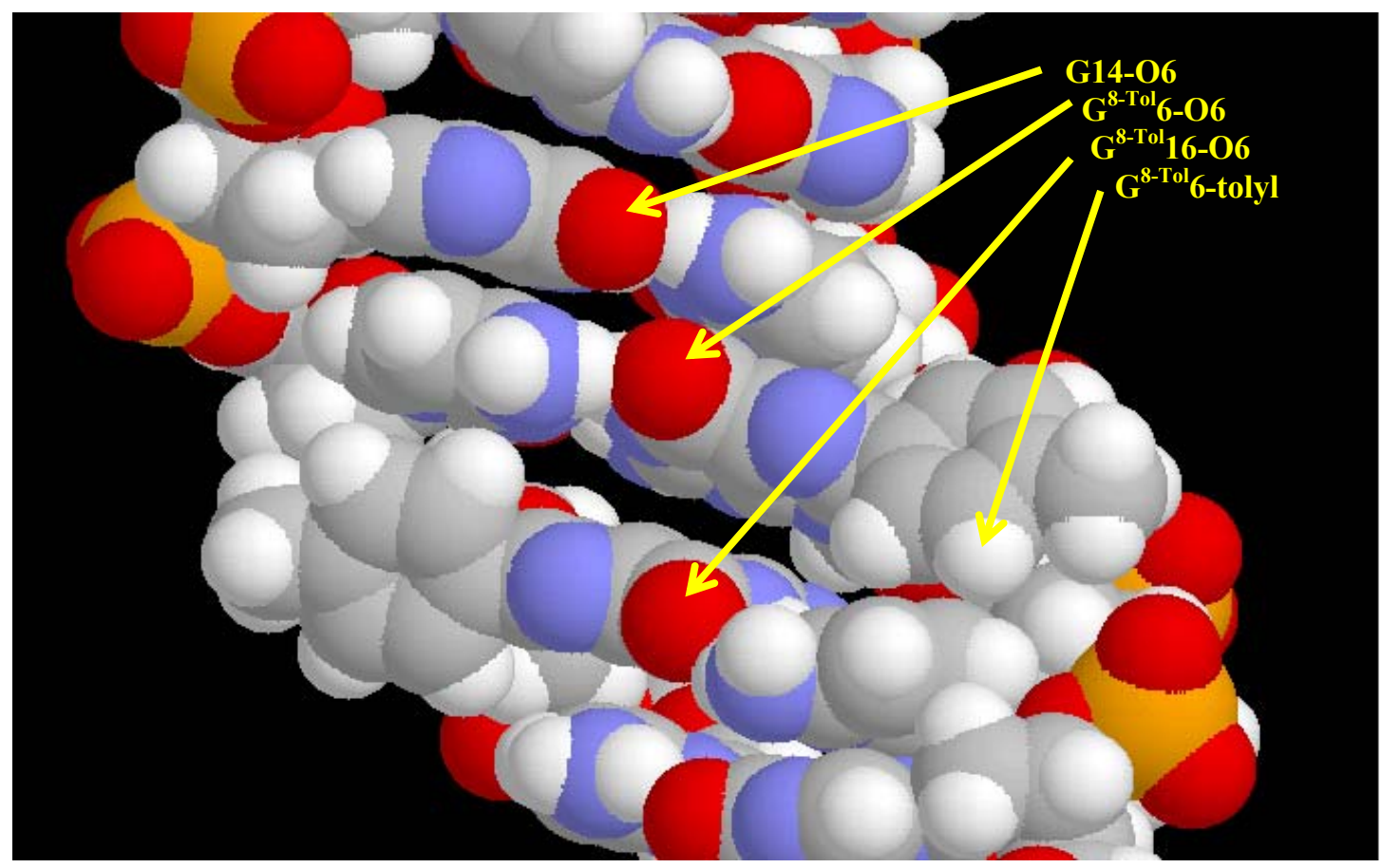

Figure 5.43. Close-up of, from bottom to top, the fourth through eighth base pair steps of the tolyl-modified B-DNA.

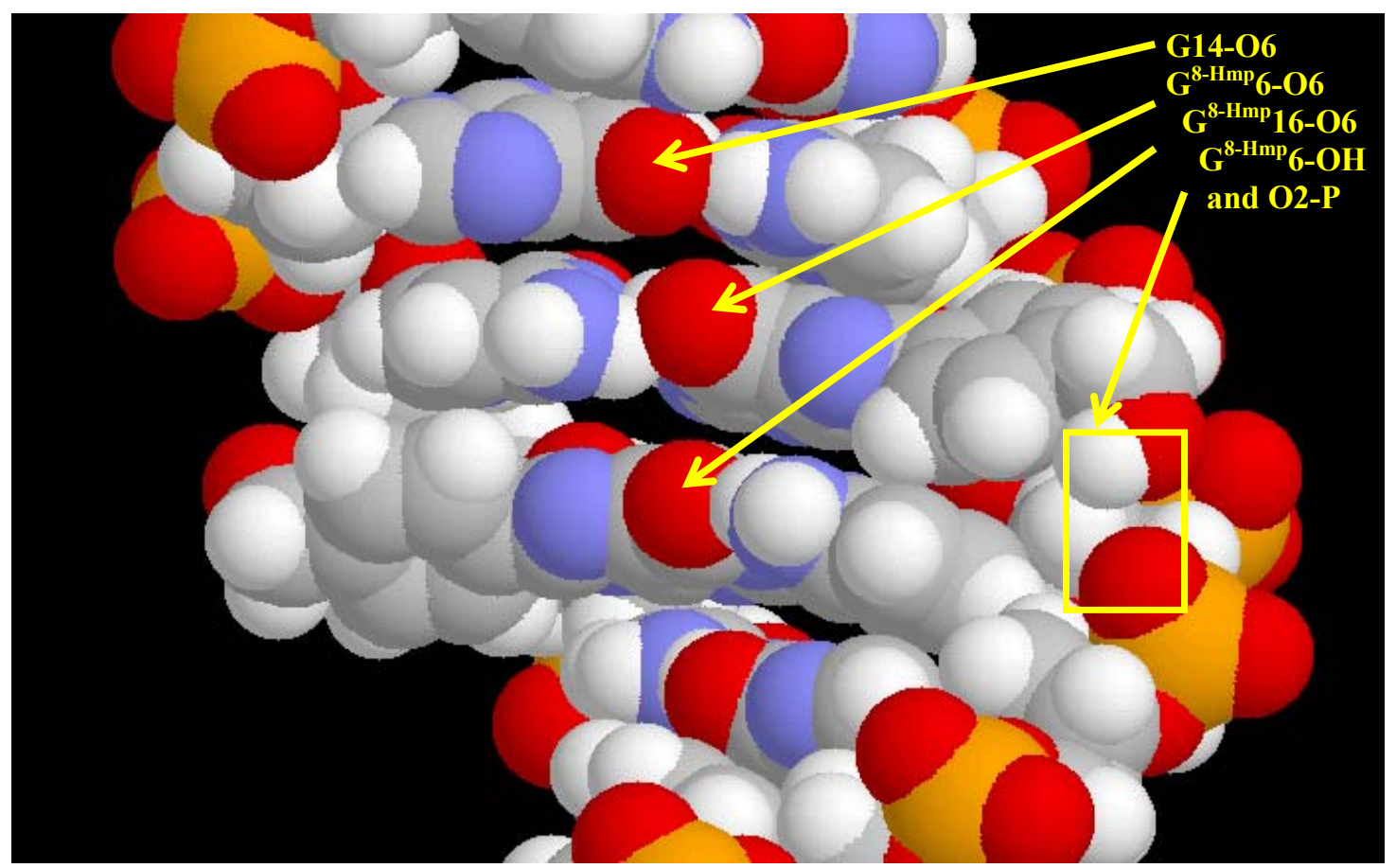

Figure 5.44. Close up of, from bottom to top, the fourth through eighth base pair steps of the hydroxymethylphenyl-modified B-DNA. 
The estimation of the solvation free energy performed using the linear PB approach compares well to those reported in the literature for a poly CG $10-$ mer $^{158}$ as shown in Table 5.17 below. There are some discrepancies in the gas phase calculations; the electrostatic and internal energy contributions are higher than those reported previously. This could be due to the use of different versions of the force field.

Table 5.17. Comparison of selected components of the free energy calculations for the unmodified B-DNA to those values found in the literature.

\begin{tabular}{|c|c|c|c|}
\hline ENERGY & CG (B) & Cheatham et al. & \% Difference \\
\hline ELE & $\begin{array}{l}-817.4 \\
(2.9)^{\mathrm{a}}\end{array}$ & $\begin{array}{l}-880.6 \\
(2.7)\end{array}$ & 7.2 \\
\hline VDW & $\begin{array}{l}-164.3 \\
(0.8)\end{array}$ & $\begin{array}{l}-159.2 \\
(0.8)\end{array}$ & 3.2 \\
\hline INT & $\begin{array}{l}939.7 \\
(1.6)\end{array}$ & $\begin{array}{l}859.7 \\
(2.2) \\
\end{array}$ & 9.3 \\
\hline GAS & $\begin{array}{l}-41.96 \\
(3.1)\end{array}$ & $\begin{array}{l}-80.1 \\
(3.1)\end{array}$ & 47.5 \\
\hline GBSUR & $\begin{array}{l}22.56 \\
(0.0)\end{array}$ & $\begin{array}{l}22.9 \\
(0.0)\end{array}$ & 1.5 \\
\hline GB & $\begin{array}{l}-4505 \\
(2.7)\end{array}$ & $\begin{array}{l}-4755 \\
(2.3)\end{array}$ & 5.2 \\
\hline TSVIB & $\begin{array}{l}490.3 \\
(0.2)\end{array}$ & 478.6 & 2.4 \\
\hline PBSOL & $\begin{array}{l}-4841 \\
(2.7)\end{array}$ & $\begin{array}{l}-4821 \\
(2.7)\end{array}$ & 0.4 \\
\hline PBELE & $\begin{array}{l}-5676 \\
(0.9)\end{array}$ & $\begin{array}{l}-5655 \\
(1.2)\end{array}$ & 0.4 \\
\hline PBTOT & $\begin{array}{l}-4883 \\
(1.5)\end{array}$ & $\begin{array}{l}-4931 \\
(1.6)\end{array}$ & 1.0 \\
\hline
\end{tabular}

${ }^{a}$ Numbers in parentheses represent the standard error of the mean.

We found some disagreement between the GB and PB results for the polar contribution to solvation. The value for the unmodified B-DNA is lower than that reported in the literature ${ }^{158}$ and the comparable value obtained from the PB approach in this work. This holds true for the modified DNA's as well. Efforts were made to understand the reason for the discrepancy and correct it. There are several "flavors", of GB available, including the "standard" pairwise 
approach used in this work, ${ }^{129}$ a modified GB model under development, ${ }^{159}$ parameterization developed by Jayaram et al., called "GB", ${ }^{160}$ and a modified parameterization of "GB" called "MGB", ${ }^{160}$ we tried several of these variations. We also experimented with different salt concentrations. Finally, we sent our data to the author of the paper used as a comparison in this work. He suggested that the PB result is preferable since the GB code is not yet stable. ${ }^{161} \mathrm{We}$, therefore, consider the PB calculations "official" and report the GB results because they were part of the learning experience of this work.

There is some question as to the reliability of the entropy calculations. Previous studies ${ }^{158}$ have shown that minimization can distort the comformation of structures. The vibrational contribution to entropy calculated by nmode for the unmodified B-DNA compared reasonably well $(490.26 \mathrm{kcal} / \mathrm{mol})$ with that reported in the literature $(478.6 \mathrm{kcal} / \mathrm{mol}) .{ }^{158}$ The overall lower entropies for the Z-DNA's relative to the B-DNA's (except $\mathbf{C G}^{\mathbf{8}-\mathrm{Hmp}}$ ) is also consistent with the known rigidity of Z-DNA in comparison to B-DNA. The relative order seems to make sense; the modifications increase the overall entropy regardless of conformation. Ignoring the entropy term, one finds the unmodified Z-DNA stabilized relative to the $\mathrm{B}$, but the free energy results for the modified DNA's remain the same. The $\mathrm{Z}$ forms of the phenyl and tolyl-modified DNA's are stabilized relative to the B, and $\mathbf{C G}^{\mathbf{8 - H m p}}$ marginally favors the B conformation.

The slight stabilization of the B conformation of the hydroxymethylphenyl-modified DNA seems to be a result of the hydrogen bonding to O2P. From a structural standpoint, $\mathbf{C G}^{\mathbf{8}}$ Hmp in the B conformation was most like the unmodified DNA, due at least in part to the hydrogen bonding, which kept the structure from moving to some other conformation both locally and globally. Because it stays closest to the unmodified B-DNA, the overall stabilizing 
force is the non-bonded electrostatic energy; it also receives more stabilization from the internal energy term relative to the other B-DNA's. When comparing the unmodified pair of confomers to the hydroxymethylphenyl pair, there is a difference in stabilizing forces. For the unmodified, it is ultimately the favorable entropy in the B conformation that drives the stability. For the HMP mofdified oligos, the more favorable internal energy for the B form and the favorable entropy for the $\mathrm{Z}$ form make the relative stability almost the same, removing the entropy term pushes the equilibrium towards the B-DNA conformation.

There are no obvious differences structurally between the tolyl-modified Z-DNA and the others; however, the energy calculations show a higher non-bonded electrostatic term and a relatively lower polar contribution to solvating free energy, which seems to counterbalance the unfavorable electrostatic term. This type of behavior is expected from Z-DNA energy analyses but it is unexpected for one in a series such as this to stand out from the others. As discussed earlier, this behavior is also observed in the tolyl-modified B-DNA but can be explained by the changes in structure brought on by the modification. One correlation between the tolyl-modified DNA's in both the B and Z conformation and their structures is the fact that in both cases they deviate most from unmodified and canonical (B and $\mathrm{Z}$ ) structures over the course of the $2 \mathrm{~ns}$ production dynamics run. The question is not so much about the end stability results but lies in the differences in energetic components within the B and Z-DNA family of structures studied here. The bottom line is the phenyl and tolyl modifications significantly stabilize the Z-DNA conformation relative to the B. The degree of stabilization is entropy dependent, but the overall stabilization is not entropy driven. In addition, the tolyl-modified DNA, which in the B form drifts the most from canonical B and unmodified B in this work, has the highest relative degree of stabilization in the Z-DNA conformation. The hydroxymethylphenyl-modified DNA, which 
in the B form is closest to canonical B and unmodified B in this work, has the least amount of relative stabilization in the Z-DNA conformation and actually favors the B form.

The modeling work discussed here correlates well with the experimental data. The melting temperatures suggest similar stability for the Z-DNA forms of $\mathbf{C G}$ and $\mathbf{C G}^{\mathbf{8}-\mathbf{P h}}$; this is further supported by the free energy calculations. The modeling data also supports what little structural information could be derived from the NMR data. The chemical shifts of the phenyl protons in both the $\mathrm{B}$ and $\mathrm{Z}$ forms are consistent with the molecular models. In fact, the models helped explain the diversity in chemical shifts attributed to the phenyl stacking with the 5' cytosine. From the models, it is easy to see that the anti conformation about the glycosidic bond places the phenyl protons in different environments, resulting in different chemical shifts. This is in contrast to the phenyl group in the Z-DNA, which sticks out away from everything else. It is also of interest to note that the tolyl derivative does not appear to form ds DNA under experimental conditions (data not shown). This novel behavoir warrants further exploration. To this end, the tolyl adduct is being examined in two additional Z-DNA forming sequences. 


\section{CHAPTER 6}

\section{Conclusion and Future Work}

\subsection{Conclusion}

There are a number of factors known to affect the relative stability of B and Z-DNA including salt concentration, counterion identity, sequence effects, base modifications, and hydrophobicity. All of these influences are important and, to some extent, overlap with one another. Here, the factors that most likely need to be weighed are base modifications, salt concentration, base stacking interactions, and hydrophobicity. C8-guanosine modification is known to stabilize the syn glycosidic bond conformation due to an unfavorable steric interaction between groups in the $\mathrm{C} 8-2$ '-deoxyguanosine position and the $\mathrm{H}-2$ " proton. This corresponds to the conformation of purines in Z-DNA, and therefore, C8-arylguanosines should stabilize ZDNA and destabilize B-DNA where the preferred glycosidic bond conformation for all unmodified bases is anti.

A second factor that may or may not destabilize the B-DNA form results from the fact that the aryl modifications lie in the major groove versus outside of the helix in the $\mathrm{Z}$ form. This could be sterically destabilizing but the resulting stacking interactions between the phenyl ring and the $5^{\prime}$ cytosine could reverse those effects. Finally, there are differences in the hydrophobic interactions of the aryl group in the B and Z-DNA forms. In the B form, the C-8-aryl group is largely shielded from the solvent (water), but it is fully exposed to solvent in the $\mathrm{Z}$ form. Thus, unlike steric effects, hydrophobic effects will tend to stabilize the B conformation relative to the Z.

Finally, the tendancy of C8-phenylguanine-modified purine/pyrimidine tracts to adopt the Z-DNA conformation may be related to the carcinogenic nature of aryl hydrazines. All of the 
aryl hydrazines we have studied have been found to produce C8-aryl purine adducts. Studies of C8-phenylguanine adducts suggest that misreading is inefficient so this type of mechanism for carcinogenesis seems unlikely. An alternative model is stabilization of the Z-DNA form. A recent study ${ }^{22}$ demonstrated that activation of a gene (CSF1) promoter requires adoption of a ZDNA structure. While unmodified Z-DNA prone sequences (alternating pyrimidine/purine sequences) adopt a B-DNA conformation under physiological conditions, C8-arylation of guanine may alter the preference and stabilize the Z-DNA structure required for gene expression. Stabilization of the $\mathrm{Z}$ form would be further enhanced if the $\mathrm{G}^{8-\mathrm{Ph}}$ adduct formed in a sequence containing $\mathrm{C}^{5-\mathrm{Me}} \cdot \mathrm{C}^{5-\mathrm{Me}}$ is known to stabilize Z-DNA in $\mathrm{CG}$ sequences ${ }^{69}$ and the level of $\mathrm{C}^{5-\mathrm{Me}}$ incorporation is correlated with gene expression. Since Z-DNA prone sequences have been identified in oncogenes such as c-Myc $\mathrm{c}^{20,21}$, it is possible the C8-arylguanine adducts formed from carcinogenic aryl hydrazines activate these genes by this mechanism.

It is of interest to note that the molecular mechanisms involved in the B to A transition and the penetration of a protein $\alpha$-helix into the major groove of DNA are the same. ${ }^{151}$ Concerted changes in backbone and glycosyl torsions result in bending, unwinding, and displacement of neighboring base pairs; the same mechanisms observed in the dynamics simulation of the modified B-DNA's to different extents. It has also been suggested that the Aform is a transient structure in the $\mathrm{B}$ to $\mathrm{Z}$ (and $\mathrm{Z}$ to $\mathrm{B}$ ) transition. ${ }^{141}$ If so, the data presented here suggests that the incorporation of $\mathrm{C} 8$-arylguanines into sequences with a propensity for Z-DNA formation facilitates such a conversion.

A-DNA, B-DNA, and Z-DNA are useful as boundary extremes in the classification of DNA but the distinction is not all or none; intermediates exist under the mildest of conditions. ${ }^{149}$ 
"We all can define extreme prototypes, but woe unto whomever shall maintain that one extreme has one set of properties, whereas the other extreme must have opposite properties."149 


\subsection{Future Work}

The NMR data is complicated due to the presence of the ss, B, and $\mathrm{Z}$ forms of DNA. In addition, since there are multiple forms of the modified oligonucleotide at low salt concentrations, the thermal denaturation data cannot be used to determine thermodynamic parameters and then compared to the corresponding unmodified oligonucleotide. We are currently exploring several alternatives that may reduce the complexity of the system. First, oligonucleotides are being prepared with the C8-phenylguanine modified base located at G8/G18. This may reduce any unfavorable interactions caused by having the modified base pairs adjacent to one another. A second approach is to use a sequence that is not selfcomplementary, so that the duplex will only contain one C8-phenylguanine but that is still prone to Z-DNA formation. A sequence meeting these criteria is ${ }^{3{ }^{\prime}} \mathrm{TGTGTG}^{8-\mathrm{Ph}} \mathrm{TGTG}^{5}$. The resulting duplex will contain only one $\mathrm{G}^{8-\mathrm{Ph}}$ modified base, and the duplex with ${ }^{3} \mathrm{CACACACACA}^{51}$ has been studied and is known to be prone to adopting a Z-DNA conformation, albeit less prone than CG. ${ }^{27,162,163,164}$ A final approach that may be explored is to stay with the same sequence and prepare oligonucleotides with other $\mathrm{C} 8$-arylguanine modifications, including $p$-tolyl, $p$ hydroxymethylphenyl, and $p$-carboxyphenyl, corresponding to the adducts that have been observed from the corresponding hydrazines in vitro and in vivo. Due to similar steric properties but significantly different hydrophobicities, these oligos may show different $B / Z$ preferences. If predilections are such that only one conformer is present, it should be possible to obtain the solution structure by NMR.

Future work from the molecular modeling standpoint includes analyzing the water/cation distribution around the DNA's in both conformations. Hydration patterns should be analyzed as these are known to be highly sequence and conformational dependent. ${ }^{165,166}$ The $\mathrm{A}, \mathrm{B}$, and $\mathrm{Z}$ 
forms of DNA are hydrated to different extents, and the geometry of hydration varies with conformation. ${ }^{165}$ Solvent (water and ions) provide stability by charge screening, through ordered water structures in the minor and major grooves that participate in hydrogen bonding with the bases, ${ }^{158}$ and by the solvation of polar groups. ${ }^{167}$ Solvent also helps to stabilize guanine in the syn conformation by forming a bridge from the $\mathrm{N} 2$ nitrogen to the phosphate oxygens of the backbone ${ }^{89}$ A complete thermodynamic characterization of the B to Z-DNA transition depends on determination of the precise role of specific water interactions. ${ }^{167}$ This work as well will benefit from analyzing such interactions.

The entropy calculations may also warrant further investigation. There is some doubt as to the validity of these calculations. The representative structures used for the computations may be improved by implicit inclusion of solvent through a continuum method such as the PB approach. ${ }^{158}$ This work uses a relatively large number of snapshots for the nmode analysis, which increases our confidence in the results relative to similar work in the literature. ${ }^{158}$ However, quasih in Amber was recommended as an alternative to nmode ${ }^{161}$ According to one of the Amber software developers, an "Achilles heel of the MM-PBSA approach is there are no really good ways to estimate entropy changes for many interesting types of transformations. This still a very active area of interest". ${ }^{168}$

An analysis of the base pair stacking energies could also provide further insight into the mechanism of stabilization for the phenyl and tolyl-modified DNA's. This information coupled with the base stacking geometry would help explain the movements of the modified base pairs, as well as provide insight into the puzzling electrostatic non-bonded and polar contribution to free energies found for the tolyl-modified DNA in both conformations. Coincidently, a major effect of the incorporation of $\mathrm{C}^{5-\mathrm{Me}}$ is an increased base stacking energy, and $\mathrm{C}^{5-\mathrm{Me}}$ substitution 
has been found to stabilize the BI substate of the B-DNA backbone relative to the BII, ${ }^{169}$ as well as the left-handed $\mathrm{Z}$ conformation as discussed previously. ${ }^{69} \mathrm{BI} / \mathrm{BII}$ (and possibly ZI/ZII) substates may have a significant role in the early stages of protein-DNA recognition. ${ }^{170}$

We also would like to model the C8-methylguanine modified oligo, as well as any alternative synthesized structures. The methyl-modified DNA is of particular interest to us because it is formed from methyl hydrazine, is carcinogenic, and shows a stronger tendency to adopt the $\mathrm{Z}$ conformation as a 6-mer than the 10-mer aryl-modified oligos in this work. ${ }^{66}$ Why this is so is not obvious but results from this work suggest that although the aryl functional groups are in a stericaly discommodious position, stacking with the $5^{\prime}$ cytosine may contribute to favorable electrostatic interactions for the B-DNA conformer. In addition, the methyl substituent is spherical and has less surface area than the planar phenyl group, which could allow it to fill a pocket on the external surface of the Z-DNA, resulting in a decrease in solvent accessible surface area. Thus, the methyl modification could cause a decrease in unfavorable hydrophobic interactions relative to unmodified and phenyl-modified Z-DNA. 


\section{References}

1) Toth, B.; Gannett, P. Agaricus Bisporus: An Assessment of Its Carcinogenic Potency. Mycopathologia 1993, 24, 73-77.

2) Gannett, P. M.; Shi, X.; Lawson, T.; Kolar, C.; Toth, B. Aryl Radical Formation During the Metabolism of Arylhydrazines by Microsomes. Chem. Res. Toxicol. 1997, 10, 1372-1377.

3) Powell, J. H.; Gannett, P. M. Mechanisms of Carcinogenicity of Aryl Hydrazines, Aryl Hydrazides, and Arenediazonium Ions. Journal of Experimental Pathology, Toxicology and Oncology 2002, 21, 1-31.

4) Kikugawa, K.; Kato, T.; Kojima, K. Substitution of $P$-and $O$-Hydroxyphenyl Radicals at the 8 Position of Purine Nucleosides by Reaction with Mutagenic $P$ and $O$-Diazoquinones. Mutat. Res. 1992, 268, 65-75.

5) Miller, H.; Prasad, R.; Wilson, S. H.; Johnson, F.; Grollman, A. 8-OxodGTP Incorporation by DNA Polymerase $\beta$ Is Modified by Active-Site Residue Asn 279. Biochem. 2000, 39, 1029-1033.

6) Tchou, J.; Grollman, A. P. Repair of DNA Containing the Oxidatively-Damaged Base, 8- Oxoguanine. Mutat. Res. 1993, 299, 277-287. 
7) Augusto, O.; Cavalieri, E. L.; Rogan, E. G.; RamaKrishna, N. V. S.; Kolar, C. Formation of 8-Methylguanine As a Result of DNA Alkylation by Methyl Radicals Generated during Horseradish Peroxidase-Catalyzed Oxidation of Methylhydrazine. J. Biol. Chem. 1990, 265, 22093-22096.

8) Augusto, O. Alkylation and Cleavage of DNA by Carbon-Centered Radical Metabolites. Fr. Rad. Biol. Med. 1993, 15, 329-336.

9) Hiramoto, K.; Kaku, M.; Kato, T.; Kikugawa, K. DNA Strand Breaking by the Carbon-Centered Radical Generated From 4-(Hydroxymethyl)Benzene Diazonium Salt, a Carcinogen in Mushroom Agaricus Bisporus. ChemicoBiological Interactions 1995, 94, 21-36.

10) Rogan, E. G.; Cavalieri, E. L.; Tibbels, S. R.; Cremonesi, P.; Warner, C. D.; Nagel, D. L.; Tomer, K. B.; Cerny, R. L.; Gross, M. L. Synthesis and Identification of Benzo[a]Pyrene-Guanine Nucleoside Adducts Formed by Electrochemical Oxidation and by Horseradish Peroxidase Catalyzed Reaction of Benzo[a]Pyrene With DNA. J. Am. Chem. Soc. 1988, 110, 4023-4029.

11) Lawson, T.; Gannett, P. M.; Yau, W.-M.; Dalal, N. S.; Toth, B. Different Patterns of Mutagenicity of Arenediazonium Ions in V79 Cells and Salmonella Typhimurium TA 102: Evidence for Different Mechanisms of Action. J. Agric. Food Chem. 1995, 43, 2627-2635. 
12) Stolarski, R.; Dudycz, L.; Shugar, D. NMR Studies on the Syn-Anti Dynamic Equilibrium in Purine Nucleosides and Nucleotides. Eur. J. Biochem. 1980, 108, 111-121.

13) Perlow, R. A.; Broyde, S. Evading the Proofreading Machinery of a Replicative DNA Polymerase: Induction of a Mutation by an Environmental Carcinogen. $J$. Mol. Biol. 2001, 309, 519-536.

14) Shane, B. S.; de Boer, J.; Watson, D. E.; Haseman, J. K.; Glickman, B. W.; Tindall, K. R. LacI Mutation Spectra Following Benz[a]Pyrene Treatment of Big Blue Mice. Carcinog. 2000, 21, 4715-4725.

15) O'Handley, S. F.; Sanford, D. G.; Xu, R.; Lester, C. C.; Hingerty, B. E.; Broyde, S.; Krugh, T. R. Structural Characterization of an N-Acetyl-2-Aminofluorene (AAF) Modified DNA Oligomer by NMR, Energy Minimization, and Molecular Dynamics. Biochem. 1993, 32, 2481-2497.

16) Kohda, K.; Tsunomoto, H.; Kasamatsu, T.; Sawamura, F.; Terashima, I.; Shibutani, S. Synthesis and Miscoding Specificity of Oligodeoxynucleotide Containing 8-Phenyl-2'-Deoxyguanosine. Chem. Res. Tox. 1997, 10, 1351-1358.

17) Gannett, P. M.; Shi, X.; Ye, J.; Powell, J. H.; Darian, E.; Daft, J. Activation of AP-1 Through the MAP Kinase Pathway: A Potential Mechanism of the 
Carcinogenic Effect of Arenediazonium Ions. Chem. Res. Toxicol. 2000, 13, 1020-1027.

18) Sugiyama, H.; Kawai, K.; Matsunaga, A.; Fujimoto, K.; Saito, I.; Robinson, H.; Wang, A. H. J. Synthesis, Structure and Thermodynamic Properties of 8Methylguanine-Containing Oligonucleotides: Z-DNA Under Physiological Salt Conditions. Nucl. Acids Res. 1996, 24, 1272-1278.

19) Gannett, P. M.; Powell, J. H.; Rao, R.; Shi, X.; Lawson, T.; Kolar, C.; Toth, B. $\mathrm{C}^{8}$-Arylguanine and $\mathrm{C}^{8}$-Aryladenine Formation in Calf Thymus DNA From Arenediazonium Ions. Chem. Res. Toxicol. 1999, 12, 297-304.

20) Wittig, B.; Wolfl, S.; Dorbic, T.; Vahrson, W.; Rich, A. Transcription of Human C-Myc in Permeabilized Nuclei Is Associated With Formation of Z-DNA in Three Discrete Regions of the Gene. EMBO J 1992, 11, 4653-4663.

21) Wolfl, S.; Wittig, B.; Rich, A. Identification of Transcriptionally Induced Z-DNA Segments in the Human C-Myc Gene. Biochim. Biophys. Acta 1995, 1264, 294302.

22) Liu, R.; Liu, H.; Chen, X.; Kirby, M.; Brown, P. O.; Zhao, K. Regulation of CSF1 Promoter by the SWI/SNF-Like BAF Complex. Cell 2001, 106, 309-318. 
23) Wang, A. H.; Quigley, G. J.; Kolpak, F. J.; Crawford, J. I.; van Bloom, J. H.; van der Marei, G. A.; Rich, A. Molecular Structure of a Left-Handed Double DNA Helical DNA Fragment at Atomic Resolution. Nature 1979, 282, 680-686.

24) Peck, L. J.; Nordheim, A.; Rich, A.; Wang, J. C. Flipping of Cloned D(PCpG)n, D(PCpG)n DNA Sequences From Right to Left-Handed Satructure by Salt, Co(III), or Negative Supercoiling. Proc. Natl Acad. Sci., USA 1982, 79, 45604564.

25) Haniford, D. B.; Pulleybank, D. E. Facile Trasition of Poly [d(TG), D(CA)] into a Left-Handed Helix in Physiological Conditions. Nature 1983, 302, 632-634.

26) Herbert, A.; Rich, A. Left-Handed Z_DNA: Structure and Function. Genetica 1999, 106, 37-47.

27) Zimmer, C.; Tymen, S.; Marck, C.; Guschlbauer, W. Conformational Transitions of Poly(dA-dC).Poly(dG-dT) Induced by High Salt or in Ethanolic Solution. Nucl. Acids Res. 1982, 10, 1081-1091.

28) Behe, M.; Felsenfeld, G.; Szu, S. C.; Charney, E.; . Temperature Dependent Conformational Transitions in Poly(dG-dC) and Poly(dG-m5dC). Biopolymers 1985, 24, 289-230. 
29) Krzyzaniak, A.; Salanski; P.; Jurczak, J.; Barciszewski, J. B-Z Reversible Conformational Changes Effected by High Pressure. FEBS Lett. 1991, 279, 1-4.

30) Stirdivant, S. M.; Klysik, J.; Wells, R. D. Energetic and Structural InterRelationship Between DNA Supercoiling and the Right- to Left-Handed Z Helix Transitions in Recombinant Plasmids. J. Biol. Chem. 2003, 257, 10159-10165.

31) Pohl, F. M.; Jovin, T. M. Salt Induced Co-Operative Conformational Change of a Synthetic DNA: Equilibrium and Kinetic Studies Woth Poly (DG-DC). J. Mol. Biol. 1972, 19, 375-396.

32) Jiang, H.; Zacharis, W.; Amirhaeri, S. Potassium Permangante As an in Situ Probe for B-Z and Z-Z Junctions. Nucl. Acids Res. 21991, 19, 6943-6948.

33) Palacek, E.; Rasvoka, E.; Boublikova, P. Probing of DNA Polymorphic Structure in the Cell With Osmium Tetraoxide. Biochem. Biophys. Res. Comm. 1988, 150, 731-738.

34) Jaworski, J.; Hsich, W. T.; Blaho, J. A.; Larson, J. E. Left-Handed DNA in Vivo. Science 1987, 238, 773-777.

35) Rahmouni, A. R.; Wells, R. D. Stabilization of Z-DNA in Vivo by Localized Supercoiling. Science 1989, 246, 358-363. 
36) Lafer, E. M.; Moller, A.; Nordheim, A.; Stollar, B. D.; Rich, A. Antibodies Specific for Left-Handed Z-DNA. Proc. Natl Acad. Sci., USA 1981, 78, 35463550.

37) Lafer, E. M.; Valle, R. P. C.; Moller, A.; Nordheim, A.; Schur, P. H.; Rich, A.; Stollar, B. D. Z-DNA Specific Antibodies in Human Systemic Lupus Erythemotis. J. Clin. Invest. 1983, 71, 314-321.

38) Wittig, B.; Dorbic, T.; Rich, A. The Level of Z-DNA in Metabolically Active, Permeabilized Mammalian Cell Nuclei Is Regulated by Torsional Strain. Journal of Cell Biology 1989, 108, 755-764.

39) Wittig, B.; Dorbic, T.; Rich, A. Transcription Is Associated With Z-DNA Formation in Metabolically Active Permeabilized Mammalian Cell Nuclei. Proc. Natl Acad. Sci., USA 1991, 88, 2259-2263.

40) Rothenberg, S.; Koch-Nolte, F.; Rich, A.; Hag, F. A Polymorphic Dinucleotide Repeat in Rat Nucleolin Gene Forms Z-DNA and Inhibits Promoter Activity. Proc. Natl Acad. Sci., USA 2001, 98, 8985-8990.

41) Bourbon, H. M.; Prudhommie, M.; Amalric, F. Sequence and Structure of the Nucleolin Promoter in Rodents: Characterization of a Strikingly Conserved CpG Island. Gene 1988, 98, 73-84. 
42) Mayer-Jung, C.; Moras, D.; Timsit, Y. Hydration and Regulation of Methylated CpG Steps in DNA. EMBO J 2003, 17, 2709-2718.

43) Herbert, A.; Spitzner, J. R.; Lowenhaupt, K.; Rich, A. Z-DNA Binding Protein From Chicken Blood Nuclei. Proc. Natl Acad. Sci., USA 1993, 90, 3339-3342.

44) Herbert, A.; Alfen, J.; Kim, Y.-G.; Mian, I. S.; Nishikura, K.; Rich, A. A Z-DNA Binding Domain Present in the Human Editing Enzyme, Double-Stranded RNA Adenosine Deaminase. Proc. Natl Acad. Sci., USA 1997, 94, 8421-8426.

45) Bass, B. L.; Weintraub, H. An Unwinding Activity that Covalently Modifies Its Double-Stranded RNA Substrate. Cell 1998, 55, 1089-1098.

46) Herbert, A.; Schade, W.; Lowenhaupt, K.; Alfken, J.; Schwartz, T.; Shlyakhtenko, L. S.; Lyubehenko, Y. L.; Rich, A. A Z-DNA Binding Domain Present in the Human ADAR1 Binds to the Z-DNA Conformer of Many Different Sequences. Nucl. Acids Res. 1998, 26, 3486-3493.

47) Schwartz, T.; Rould, M.; Lowenhaupt, K.; Herbert, A.; Rich, A. Crystal Structure of the Z $\alpha$ Domain of the Human Editing Enzyme ADAR1 Bound to Left-Handed Z-DNA. Science 1999, 284, 1841-1845. 
48) Schwartz, T.; Behike, J.; Lowenhaupt, K.; Heinemann, U.; Rich, A. Structure of the DLM-1-Z-DNA Complex Reveals a Conserved Family of Z-DNA-Binding Proteins. Nature Structural Biology 2001, 8, 761-765.

49) Rothenberg, S.; Schwartz, T.; Koch-Nolte, F.; Haag, R. Complex Regulation of the Human Gene for the Z-DNA Binding Protein DLM-1. Nucl. Acids Res. 2002, 30, 993-2000.

50) Oh, K. B.; Kim, Y.-G.; Rich, A. Z-DNA Binding Proteins Can Act as Potent Effectors of Gene Expression in Vivo. Proc. Natl Acad. Sci., USA 2002, 99, 16666-16671.

51) Gannett, P. M.; Heavner, S.; Daft, J. R.; Shaughnessy, K. H.; Epperson, J. D.; Greenbaum, N. L. Synthesis, Properties, and NMR Studies of a C8Phenylguanine Modified Oligonucleotide That Preferentially Adopts the Z-DNA Conformation. Chem. Res. Toxicol. 2003, 1385-1394.

52) Szklarz, G.; Halpert, J. R. Use of Homology Modeling in Conjunction with SiteDirected Mutagenesis for Analysis of Structure-Function Relationships of Mammalian Cytochromes P450. Life Sciences 1997, 61, 2507-2520.

53) Stefl; Cheatham, T. E., III; Spackova, N.; Fadrna; Berger, I.; Kuca, J.; Sponer, J. Formation Pathways of a Guanine-Quadruplex DNA Revealed by Molecular 
Dynamics and Thermodynamics Analysis of Substrates. Biophys. J. 2003, 80, $455-468$.

54) Spackova, N.; Cheatham, T. E., III; Ryjacek, F.; Lankas, F.; Van Meervelt, L.; Hobza, P.; Sponer, J. Molecular Dynamics Simulations and Thermodynamics Analysis of DNA-Drug Complexes. Minor Groove Binding Between 4',6Diamidino-2-Phenylindole and DNA Duplexes in Solution. J. Am. Chem. Soc. 2003, 125, 1759-1769.

55) Darian, E. Triplex Formation as Monitored by EPR Spectroscopy and Molecular Dynamics Studies of Spin-probe Labeled DNAs. 66-112. 2002. West Virginia University ( http://etd.wvu.edu//ETDS/E2591/Darian_Eva_dissertation.pdf $)$.

56) Nilsson, L. Protein-Nucleic Acid Interaction. In Encylclopedia of Computational Chemistry; P.V.R.Schleyer, Ed.; Wiley: New York, 1998.

57) Jain, S.; Zon, G.; Sundaralingam, M. The Potentially Z-DNA forming Sequence d(GTGTACAC) Crystalizes as A-DNA. J. Mol. Biol. 197, 141-145. 1987.

58) Hunter, C. A. Sequence-Dependent DNA Structure. The Role of Base Stacking Interactions. J. Mol. Biol. 1993, 230, 1025-1054.

59) Cornell, W. D.; Cieplak, P.; Bayly, C. I.; Gould, I. R.; Merz, Jr. K. M.; Ferguson, D. M.; Spellmeyer, D. C.; Fox, T.; Caldwell, J. W.; Kollman, P. A. A Second 
Generation Force Field for the Simulation of Proteins, Nucleic Acids, and Organic Molecules. J. Am. Chem. Soc. 1999, 117, 5179-5197.

60) Western, E. C.; Daft, J. R.; Johnson, I. E. M.; Gannett, P. M.; Shaughnessy, K. H. Efficient One-Step Suzuki Arylation of Unprotected Halonucleosides Using Water Soluuble Palladium Catalysts. J. Org. Chem. 2003, 68, 6767-6774.

61) Techniques Applied to Nucleic Acids. In Nucleic Acids in Chemistry and Biology; Blackburn, G. M., Gait, J., Eds.; Oxford University Press: New York, 1997; Chapter 10.

62) Gannett, P. M. Thermal Denaturation Experimental Conditions. 11-18-2003.

63) van Houte, L. P. A.; Westra, J. G.; van Grondelle, R. A Spectroscopic Study of the Conformation of Poly d(G-C).Poly d(G-C) Modified With the Carcinogen 2Aminofluorene. Carcingenesis 1988, 9, 1017-1027.

64) Ornstein, R. L.; Rein, R.; Breen, D. L.; MacElroy, R. D. An Optimized Potential Function for the Calculation of Nucleic Acid Interaction Energies. I. Base Stacking. Biopolymers 1978, 17, 2341-2360.

65) Fabrega, C.; Macias, M. J.; Eritja, R. Synthesis and Properties of Oligonucleotides Containing 8-Bromo-2'-Deoxyguanosine. Nucleosides, Nucleotides, and Nucleic Acids 2001, 20, 251-260. 
66) Kohda, K.; Tsunomoto, H.; Minoura, Y.; Tanaba, K.; Shibutani, S. Synthesis, Miscoding Specificity, and Thermodynamic Stability of Oligodeoxynucleotide Containing 8-Methyl-2'-Deoxyguanosine. Chem. Res. Toxicol. 1996, 9, 12781284.

67) Kohda, K.; Tsunomoto, H.; Kasamatsu, T.; Sawamura, F.; Terashima, I.; Shibutani, S. Synthesis and Miscoding Specificity of Oligodeoxynucleotide Containing 8-Phenyl-2'-Deoxyguanosine. Chem. Res. Toxicol. 1997, 10, 13511358.

68) Markey, L. A.; Breslauer, K. J. Calculating Thermodynamic Data for Transitions of Any Molecularity from Equilibrium Melting Curves. Biopolymers 1987, 26, 1601-1620.

69) Behe, M.; Felsenfeld, G. Effects of Methylation on a Synthetic Polynucleotide: The B-Z Transition in Poly $\left(\mathrm{dG}-\mathrm{m}^{5} \mathrm{dC}\right) \cdot \operatorname{Poly}\left(\mathrm{dG}-\mathrm{m}^{5} \mathrm{dC}\right)$. Biochem. 1981, 23, 54-62.

70) CD of Nucleic Acids. In Circular Dichroism: Principles and Applications. 2nd Edition; Berova, N., Nakanishi, K., Woody, R. W., Eds.; A John Wiley \& Sons, Inc: New York, Chichester, Weinheim, Brisbane, Singapore, Toronto, 2000; Chapter 24, 25.

71) Circular Dichroism: An Introduction. In Circular Dichroism: Principles and Applications. 2nd Edition; Berova, N., Nakanishi, K., Woody, R. W., Eds.; A 
John Wiley \& Sons, Inc: New York, Chichester, Weinheim, Brisbane, Singapore, Toronto, 2000; Chapter 1.

72) Möller, A.; Nordheim, A.; Kozlowski, S.; Patek, J.; Rich, A. Bromination Stabilizes Poly(dG-dC) in the Z-DNA Form Under Low-Salt Conditions. Biochem. 1984, 23, 54-62.

73) Goto, S. Characterization of Intermediate Conformational States in the $B<->Z$ Transitions of Poly(dG-dC) Poly(dG-dC). Biopolymers 1984, 23, 2211-2222.

74) Chen, Q.; Kuntz, I. D.; Shafer, R. H. Spectroscopic Recognition of Guanine Dimeric Hairpin Quadruplexes by a Carbocyanine Dye. Proc. Natl. Acad. Sci., USA 1996, 93, 2635-2639.

75) Herbert, A.; Schade, W.; Lowenhaupt, K.; Alfken, J.; Schwartz, T.; Shlyakhtenko, L. S.; Lyubehenko, Y. L.; Rich, A. The Z $\alpha$ Domain From Human ADAR1 Binds to the Z-DNA Conformer of Many Different Sequences. Nucl. Acids Res. 1998, 26, 3486-3493.

76) Johnson, W. C. CD of Nucleic Acids. In Circular Dichroism: Principles and Applications; Berova, N., Nakanishi, K., Woody, R. W., Eds.; John Wiley and Sons, Inc: New York, 2000; Chapter 24. 
77) Gray, D.; Ratliff, R.; Vaughan, M. Circular Dichroism Spectroscopy of DNA. Meth. Enzymol. 1992, 211, 389-405.

78) Blackburn, E. H. Telomeres. Trends Biochem. Science 1991, 16, 378-381.

79) Techniques Applied to Nucleic Acids. In Nucleic Acids in Chemistry and Biology; G.Michael Blackburn, Michael J.Gait, Eds.; Oxford Press: New York, 1997; Chapter 10.

80) Scheek, R. M.; Boelens, R.; Russo, N.; van Boom, J. H.; Kaptein, R. Sequential Resonance Assignments in ${ }^{1} \mathrm{H}$ NMR Spectra of Oligonucleotides by TwoDimensional NMR Spectroscopy. Biochem. 1984, 23, 1371-1376.

81) Feigon, J.; Sklenar, V.; Wang, E.; Gilbert, D. E.; Macaya, R. F.; Schultze, P. ${ }^{1} \mathrm{H}$ NMR Spectroscopy of DNA. Meth. Enzymol. 1992, 211, 235-253.

82) Mitra, C. K.; Sarma, M. H.; Sarma, R. H. Left-Handed Deoxyribonucleic Acid Double Helix in Solution. Biochem. 1981, 20, 2036-2041.

83) Feigon, J.; Wang, A. H. J.; van der Marei, G. A.; van Boom, J. H.; Rich, A. A One- and Two-Dimensional NMR Study of the B to Z Transition of $\left(\mathrm{M}^{5} \mathrm{dC}-\mathrm{dG}\right)_{2}$ in Methanolic Solution. Nucl. Acids Res. 1984, 12, 1243-1263. 
84) Mao, B.; Hingerty, B. E.; Broyde, S.; Patel, D. J. Solution Structure of the Aminofluorene [AF]-Intercalated Conformer of the $S y n-[\mathrm{AF}]-\mathrm{C}^{8}-\mathrm{dG}$ Adduct Opposite dC in a DNA Duplex. Biochem. 1998, 37, 81-94.

85) Altona, C.; Sundaralingam, M. Conformational Analysis of the Sugar Ring in Nucleosides and Nucleotides. A New Description Using the Concept of Pseudorotation. J. Am. Chem. Soc. 1972, 94, 8205-8212.

86) Abuaf, P.; Kadlubar, F. F.; Grunberger, D. Circular Dichroism of Poly(dG-dC) Modified by the Carcinogens N-Methyl-4-Aminoazobenzene or 4Aminobiphenyl. Nucl. Acids Res. 1987, 15, 7125-7136.

87) Fazakerley, G. V.; van der Marel, G. A.; van Boom, J. H.; Guschlbauer, W. Helix Opening in Deoxyribonucleic Acid From a Proton Nuclear Magnetic Resonance Study of Imino and Amino Protons in d(CG)3. Nucl. Acids Res. 1984, 12, 82698279.

88) Orbons, L. P. M.; van der Marel, G. A.; van Boom, J. H.; Altona, C. The B and Z Forms of the $\mathrm{d}\left(\mathrm{m}^{5} \mathrm{C}-\mathrm{G}\right)_{3}$ and $\mathrm{d}\left(\mathrm{Br}^{5} \mathrm{C}-\mathrm{G}\right)_{3}$ Hexamers in Solution. A 300-MHz and 500-MHz Two Dimensional NMR Study. Eur. J. Biochem. 1986, 160, 131-139.

89) Eichman, B. F.; Schroth, G. P.; Basham, B. E.; Ho, P. S. The Intrinsic Structure and Stability of Out-of-Alteration Base Pairs in Z-DNA. Nucl. Acids Res. 1999, $27,543-550$. 
90) Ansevin, A. T.; Wang, A. H. Evidence for a New Z-Type Left-Handed DNA Helix: Properties of Z(WC)-DNA. Nucl. Acids Res. 1990, 18, 6119-6126.

91) Case, D. A.; Pearlman, D. A.; Caldwell, J. W.; Cheatham, T. E., III; Ross, W. S.; Simmerling, C. L.; Darden, T. A.; Merz, K. M.; Stanton, R. V.; Chen, A.; Vincent, J. J.; Crowley, M.; Tsui, V.; Radmer, R. J.; Duan, Y.; Pitera, J.; Massova, I.; Seibel, G. L.; Singh, U. C.; Weiner, P. K.; Kollman, P. A. Amber 6. 2000. University of California, San Francisco.

92) Case, D. A.; Pearlman, D. A.; Caldwell, J. W.; Cheatham, T. E., III; Wang, W. S.; Ross, W. S.; Simmerling, C. L.; Darden, T. A.; Merz, K. M.; Stanton, A. L.; Cheng, J. J.; Crowley, V. M.; Tsui, V.; Gohlke, H.; Radmer, R. J.; Duan, Y.; Pitera, J.; Massova, I.; Seibel, G. L.; Singh, U. C.; Weiner, P. K.; Kollman, P. A. AMBER 7. 2002. University of California, San Francisco.

93) Schlick, T. Molecular Modeling and Simulation: An Interdisciplinary Guide; Springer Verlag: New York, 2002.

94) Allinger, N. L.; Sprague, J. T. Calculation of the Structures of Hydrocarbons Containing Delocalized Electronic Systems by the Molecular Mechanics Method. J. Am. Chem. Soc. 1973, 95, 3893-3907.

95) McCammon, J. A.; Gelin, B. R.; Karplus, M. Dynamics of Folded Proteins. Nature 1977, 267, 585-590. 
96) Weiner, P. K.; Kollman, P. A. Amber: Assisted Model Building With Energy Refinement. a General Program for Modeling Molecules and their Interactions. $J$. Comp. Chem. 1981, 2, 287-303.

97) Brooks, B. R.; Bruccoleri, R.E..; Olafson, B. D.; States, D. J.; Swaminathan, S.; Karplus, M. CHARMM: A Program for Macromolecular Energy, Minimization, and Dynamics Calculations. J. Comp. Chem 1983, 4, 187-217.

98) Richon, A. B. A Scrolling History of Computational Chemistry. 2001. http://www.netsci.org/Science/Compchem/feature17b.html.

99) MacKerell, Jr. A. D.; Wiorkiewicz-Kuczera, J.; Karplus, M. An All-Atom Emperical Energy Function for the Simulation of Nucleic Acids. J. Am. Chem. Soc. 1995, 117, 11946-11975.

100) Cheatham, T. E., III; Kollman, P. A. Observation of the A-DNA to B-DNA Transition During Unrestrained Molecular Dynamics in Aqueous Solution. $J$. Mol. Biol. 1996, 259, 434-444.

101) Sheilds, G. C.; Laughton, C. A.; Orozco, M. Molecular Dynamics Simulations of the $\mathrm{D}(\mathrm{T} \cdot \mathrm{A} \cdot \mathrm{T})$ Triple Helix. J. Am. Chem. Soc. 2996, 119, 7463-7469. 
102) Cheatham, T. E., III; Crowley, M. F.; Fox, T.; Kollman, P. A. A Molecular Level Picture of the Stabilization of A-DNA in Mixed Ethanol-Water Solutions. Proc. Natl Acad. Sci., USA 1997, 94, 9626-9630.

103) Kuhn, B.; Kollman, P. A. Binding of a Diverse Set of Ligands to Avidin and Streptavidin: An Accurate Quantitative Prediction of Their Relative Affinities by a Combination of Molecular Mechanics and Continuum Solvent Models (MM/PBSA). J. Med. Chem. 2000, 343, 3786-3791.

104) Srinivasan, J.; Miller, J.; Kollman, P. A.; Case, D. A. Continuum Solvent Studies of the Stability of RNA Hairpin Loops and Helices. J. Biomol. Struc. Dyn. 1998, 16, 671-682.

105) Reyes, C.; Kollman, P. A. Structure and Thermodynamics of RNA-Protein Binding: Using Molecular Dynamics and Free Energy Analyses to Calculate the Free Energies of Binding and Conformational Change. J. Mol. Biol. 2000, 297, $1145-1158$.

106) M.J.Frisch; G.W.Trucks; H.B.Schlegel; G.E.Scuseria; M.A.Robb. Gaussian 98 (Revision A.x). 1998. Pittsburgh PA, Gaussian Inc.

107) Cheatham, T. E., III; Brooks, B. R.; Kollman, P. A. Molecular Modeling of Nucleic Acid Structure. In Current Protocols in Nucleic Acid Chemistry; Wiley: New York, 2000; Chapter 7. 
108) Weiner, S. J.; Kollman, P. A.; Case, D. A.; Singh, U. C.; Ghio, C.; Alagona, G.; Profeta, S. Jr.; Weiner, P. A New Force Field for Molecular Mechanical Simulatin of Nucleic Acids and Proteins. J. Am. Chem. Soc. 1984, 106, 765-784.

109) Allen, M. P.; Tildesley, D. J. Computer Simulation of Liquids; Oxford University Press: Oxford, 1987.

110) Brooks, C. L. I.; Karplus, M.; Pettitt, B. M. Proteins. A Theoretial Perspective of Dynamics, Structure, and Thermodynamics. In Advances in Chemical Physics; Prigogine, I., Rice, S. A., Eds.; John Wiley \& Sons: New York, 1988; Chapter 71.

111) Becker, O. M.; Watanabe, M. Dynamics Methods. In Computational Biochemistry and Biophysics; Becker, O. M., MacKerell, Jr. A. D., Roux, B., Watanabe, M., Eds.; Marcel Dekker: New York, 2001; Chapter 3.

112) Seibel, G. L.; Singh, U. C.; Kollman, P. A. A Molecular Dynamics Simulation of Double-Helical B-DNA Including Counterions and Water. Proc. Natl Acad. Sci., USA 1985, 82, 6537-6540.

113) Beveridge, D. L.; Ravishankar, G. Cur. Opt. Struct. Biol. 1994, 4, 246-255.

114) Cheatham, T. E., III; Kollman, P. A. Observation of the A-DNA to B-DNA Transition During Unrestrained Molecular Dynamics in Aqueous Solution. $J$. Mol. Biol. 1996, 259, 434-444. 
115) Cheatham, T. E., III; Miller, J. L.; Fox, T.; Darden, T. A.; Kollman, P. A. Molecular Dynamics Simulations on Solvated Biomolecular Systems - The Particle Mesh Ewald Method Leads to Stable Trajectories of DNA, RNa and Proteins. J. Am. Chem. Soc. 1995, 117, 4193-4194.

116) Jorgensen, W. L.; Chandrasekhar, J.; Machura, J.; Klein, M. L. Comparison of Simple Potential Functions for Simlating Liquid Water. J. Phys. Chem. 1983, 79, $926-935$.

117) MacKerell, Jr. A. D.; Nilsson, L. Nucleic Acid Simulations. In Computational Biochemistry and Biophysics; Becker, O., MacKerell, Jr. A. D., Roux, B., Watanabe, M., Eds.; Marcel Dekker, Inc.: New York, 2001; Chapter 20.

118) Berendsen, H. J. C.; Postma, J. P. M.; van Gunsteren, W. F.; DiNola, A.; Haak, J. R. Molecular Dynamics With Coupling to an External Bath. J. Phys. Chem. 1984, $81,3684-3690$.

119) Demchuk, E.; Bashford, D.; Gippert, G. P.; Case, D. A. Thermodynamics of a Reverse Turn Motif. Solvent Effects and Side-Chain Packing. J. Mol. Biol. 1997, 270, 305-317.

120) Yang, A. S.; Hitz, B.; Honig, B. Free Energy Determinants of Secondary Structure Formation: III. Beta-Turns and Their Role in Protein Folding. J. Mol. Biol. 1996, 259, 873-882. 
121) Yang, A. S.; Honig, B. Free Energy Determinants of Secondary Structure Formation: I. Alpha-Helices. J. Mol. Biol. 1995, 252, 351-365.

122) Bashford, D.; Case, D. A.; Choi, C.; Gippert, G. P. A Computational Study of the Role of Solvation Effects in Reverse Turn Formation in the Tetrapeptides APGD and APGN. J. Am. Chem. Soc. 1997, 119, 4964-4971.

123) Cheatham, T. E., III; Srinivasan, J.; Case, D. A.; Kollman, P. A. Molecular Dynamics and Continuum Solvent Studies of the Stability of PolyG-PolyC and PolyA-PolyT DNA Duplexes in Solution. J. Biomol. Struc. Dyn. 1998, 16, 671682.

124) Honig, B.; Sharp, K.; Yang, A. S. Macroscopic Models of Aqueous Solutions: Biological and Chemical Applications. J. Phys. Chem. 1993, 97, 1101-1109.

125) Rocchia, W.; Alexov, E.; Honig, B. Extending the Application of the Nonlinear Poisson-Boltman Equation, Multiple Dielectric Constants and Multivalent Ions. $J$. Phys. Chem., B 2001, 105, 6507-6514.

126) Gilson, M.; Sharp, K.; Honig, B. Calculating the Electrostatic Potential of Molecules in Solution: Method and Error Assessment. J. Comp. Chem 1987, 9 , 327-335. 
127) Still, W. C.; Tempczyk, A.; Hawley, R. C.; Hendrickson, J. Semianalytical Treatment of Solvation for Molecular Mechanics and Dynamics. J. Am. Chem. Soc. 1990, 112, 6127-6129.

128) Srinivasan, J.; Cheatham, T. E., III; Cieplak, P.; Kollman, P. A.; Case, D. A. Continuum Solvent Studies of the Stability of DNA, RNA, and PhosphoramidateDNA Helices. J. Am. Chem. Soc. 1998, 120, 9401-9409.

129) Tsui, V.; Case, D. A. Molecular Dynamics Simulations of Nucleic Acids with a Generalized Born Solvation Model. J. Am. Chem. Soc. 2000, 122, 2489-2498.

130) Sharp, K.; Honig, B. Electrostatic Interactions in Macromolecules: Theory and Applications. Ann. Rev. Biophys. Biophys. Chem. 1990, 19, 301-332.

131) Sitkoff, D.; Sharp, K.; Honig, B. Accurate Calculation of Hydration Free Energies Using Macroscopic Solvent Models. J. Phys. Chem. 1994, 98, 1978-1988.

132) Sanner, M.; Olson, A. J.; Spehner, J. C. Reduced Surface: An Efficient Way to Compute Molecular Surfaces. Biopolymers 1996, 38, 320.

133) Kollman, P. A.; Massova, I.; Reyes, C.; Kuhn, B.; Huo, S.; Chong, L.; Lee, M.; Lee, T.; Duan, Y.; Wang, W.; Donini, O.; Cieplak, P.; Srinivasan, A. R.; Case, D. A.; Cheatham, T. E., III Calculating Structures and Free Energies of Complex 
Molecules: Combining Molecular Mechanics and Continuum Models. Accts Chem. Res. 2000, 33, 889-897.

134) Coleman, W. F.; Arumainayagam, C. R. HyperChem 5 (by HyperCube, Inc.). Journal of Chemical Education 75, 416. 1998.

135) Wang, J.; Wang, W.; Kollman, P. A. Antechamber, an Acessory Software Package for Molecular Mechanical Calculations. 2003.

136) Allen; F.H. The Cambridge Structural Database: a Quarter of a Million Crystal Structures and Rising. Acta Cryst. 2002, B58, 380-388.

137) Lavery, R.; Sklenar, V. The Definition of Generalized Helicoidal Parameters and of Axis Curvature for Irregular Nucleic Acids. J. Biomol. Str. Dyn. 1988, 6, 6391.

138) Stofer, E.; Lavery, R. Measuring the Geometry of DNA Grooves. Biopolymers 1994, 34, 337-346.

139) Ravishanker, G. Swaminathan, S., Beveridge, D. L., Lavery, R. and Sklenar, H. Conformational and Helicoidal Analysis of 30 ps of Molecular Dynamics on the d(CGCGAATTCGCG) Double Helix: "Curves", Dials and Windows. J. Biomol. Struc. Dyn. 6, 669. 1989. 
140) Simmerling, C. L.; Elber, R.; Zhang, J. MOIL-View - A Program for Visualization of Structure and Dynamics of Biomolecules and STO - A Program for Computing Stochastic Paths. In Modeling of Biomolecular Structure and Mechanisms; Pullman, A., Ed.; Kluwer: Netherlands, 1995.

141) Arnott, S. Polynucleotide Secondary Structures: An Historical Perspective. In Oxford Handbook of Nucleic Acid Structure; Neidle, S., Ed.; Oxford University Press: New York, 1999; Chapter One.

142) Olson, W. K.; Bansai, M.; Burley, S. K.; Dickerson, R. E.; Gerstein, M.; Harvey, S. C.; Heinemann, U.; Lu, X.-J.; Neidle, S.; Shakked, Z.; Sklenar, H.; Suzuki, M.; Tung, C.-S.; Westhof, E.; Wolberger, C.; Berman, H. M. A Standard Reference Frame for the Description of Nucleic Acid Base-Pair Geometry. J. Mol. Biol. 2001, 313, 229-237.

143) DNA and RNA Structure In Nucleic Acids in Chemistry and Biology; Blackburn, G. M., Gait, J., Eds.; Oxford University Press: New York, 1997; Chapter 10.

144) Slickers, P. Measuring the Groove Width. http://www.imbjena.de/Piet/help/groovewidth.html . 1999. Germany, IMB jena.

145) Lavery, R.; Zakrzewska, K. Base and Base Pair Morphologies, Helical Parameters and Definitions. In Qxford Handbook of Nucleic Acid Structure; Neidle, S., Ed.; Oxford University Press: New York, 1999; Chapter 2. 
146) Kielkopf, C. L.; Erikka, K. E.; Hudson, B. P.; Barton, J. K. Structure of a Photoactive Rhodium Complex Intercalated into DNA. Nature Structural Biology 2000, 7, 117-121.

147) Boutonnet, N.; Hui, X.; Zakrzewska, K. Looking into the Grooves of DNA. Biopolymers 1993, 33, 479-490.

148) Dickerson, R. E.; Drew, H. R.; Conner, B. N.; Wing, R. M.; Fratini, A. V.; Kopka, M. L. The Anatomy of A-, B-, and Z-DNA. Science 1982, 216, 475-484.

149) Dickerson, R. E.; Ng, H. L. DNA Structure from A to B. Proc. Natl. Acad. Sci., USA 2001, 98, 6986-6988.

150) Vargason, J.; Eichman, B. F.; Ho, P. S. The Extended and Eccentric E-DNA Structure Induced by Cytosine Methylation or Bromination. Nature Structural Biology 2000, 7, 758-761.

151) Olson, W. K.; Gorin, A. A.; Lu, X.-J.; Hock, L. M.; Zhurkin, V. B. DNA Sequence-Dependent Deformability Deduced From Protein-DNA Crystal Complexes. Proc. Natl. Acad. Sci., USA 1998, 95, 11163-11168.

152) Tsui, V.; Case, D. A. Molecular Dynamics Simulations of Nucleic Acids with a Generalized Born Solvation Model. J. Am. Chem. Soc. 2000, 122, 2489-2498. 
153) David, L.; Luo, R.; Gilson, M. K. Comparison of Generalized Born and Poisson Models: Energetics and Dynamics of HIV Protease. J. Comp. Chem. 2000, 21, 295-309.

154) Cornell, W. D.; Cieplak, P.; Bayly, C. I.; Gould, I. R.; Merz, K. M.; Ferguson, D. M.; Spellmeyer, D. C.; Fox, T.; Caldwell, J. W.; Kollman, P. A. A Second Generation Force Field for the Simulation of Proteins, Nucleic Acids, and Organic Molecules. J. Am. Chem. Soc. 1995, 117, 5179-5197.

155) Grzeskowiak, K.; Yanag, K.; Prive, G.; Dickerson, R. E. The Structure of BHelical CGATCGATCG and Comparison with CCAACGTTGG. J. Biol. Chem. 1991, 266, 8861-8883.

156) Mazur, J.; Jernigan, R. L.; Sarai, A. Conformational Effects of DNA Stretching. Mol. Biol. (Russian) 2003, 37, 277-287.

157) Martz, E. Protein Explorer: Easy yet Powerful Macromolecular Visualization. Trends in Biochemical Sciences 2002, 27, 107-109.

158) Cheatham, T. E., III; Srinivasan, J.; Case, D. A.; Kollman, P. A. Molecular Dynamics and Continuum Solvent Studies of the Stability of PolyG-PolyC and PolyA-PolyT DNA Duplexes in Solution. Journal of Biomolecular Structure \& Dynamics 1998, 16, 265-280. 
159) Onufriev, A.; Bashford, D.; Case, D. A. Modification of the Generalized Born Model Suitable for Macromolecules. J. Phys. Chem. B 2000, 104, 3712-3720.

160) Jayaram, B.; Sprous, D.; Young, M. A.; Beveridge, D. L. Free Energy Analysis of the Conformation Preferences of A and B Forms of DNA in Solution. J. Am. Chem. Soc. 1998, 120, 10629-10633.

161) Cheatham, T. E., III Generalized Born and Poisson Boltzman Energy Calculations. 7-4-2003.

162) Thomas, T. J.; Messner, R. P. A Left-Handed (Z) Conformation of Poly(dA-dC) Poly(dG-dT) Induced by Polyamines. Nucl. Acids Res. 1986, 14, 6721-6733.

163) Riazance-Lawrence, J. H.; Johnson, W. C., Jr. Multivalent Ions Are Necessary for Poly[d(AC) $d(\mathrm{GT})]$ to Assume the Z Form: A CD Study. Biopolymers 1992, 32, 271-276.

164) Reich, Z.; Friedman, P.; Levin-Zaidman, S.; Minsky, A. Effects of Adenine Tracts on the B-Z Transition. J. Biol. Chem. 1993, 268, 8261-8266.

165) Schneider, B.; Patel, K.; Berman, H. M. Hydration of the Phosphate Group in Double-Helical DNA. Biophys. J. 1998, 75, 2422-2434. 
166) Barciszewski, J.; Jurczak, J.; Porowski, S.; Specht, T.; Erdmann, V. A. The Role of Water in Conformational Changes in Nucleic Acids in Ambient and HighPressure Conditions. Eur. J. Biochem. 1999, 260, 293-307.

167) Misra, V. K.; Honig, B. The Electrostatic Contribution to the B to Z Transition of DNA. Biochem. 1996, 35, 1115-1124.

168) Case, D. A. Entropy Estimation of DNA Using Nmode. AMBER Archive. 2000.

169) Rauch, C.; Trieb, M.; Wellenzohn, B.; Loferer, M.; Voegele, A.; Wibowo, F. R.; Liedl, K. R. C5-Methylation of Cytosine in B-DNA Thermodynamically and Kinetically Stabilizes BI. J. Am. Chem. Soc. 2003, 125, 14990-14991.

170) Varnai, P.; Djuranovic, D.; Lavery, R.; Hartmann, B. $\alpha / \gamma$ Transitions in the BDNA Backbone. Nucl. Acids Res. 2002, 30, 5398-5406. 


\section{Appendix A}

\section{Sample Gaussian Input File for use with RESP}

\begin{tabular}{|c|c|c|c|c|c|c|}
\hline \multicolumn{7}{|c|}{$\begin{array}{c}\text { \# B3LYP/6-31C } \\
\text { \# hmg_gaus }\end{array}$} \\
\hline \multicolumn{7}{|l|}{01} \\
\hline \multicolumn{7}{|l|}{$\mathrm{N}$} \\
\hline $\mathrm{C}$ & 1 & B1 & & & & \\
\hline $\mathrm{N}$ & 2 & B2 & 1 & A1 & & \\
\hline $\mathrm{C}$ & 3 & B3 & 2 & $\mathrm{~A} 2$ & 1 & D1 \\
\hline $\mathrm{C}$ & 4 & B4 & 3 & A3 & 2 & D2 \\
\hline $\mathrm{O}$ & 5 & B5 & 4 & $\mathrm{~A} 4$ & 3 & D3 \\
\hline $\mathrm{N}$ & 5 & B6 & 4 & A5 & 3 & D4 \\
\hline $\mathrm{H}$ & 7 & B7 & 5 & A6 & 4 & D5 \\
\hline $\mathrm{C}$ & 7 & B8 & 5 & A7 & 4 & D6 \\
\hline $\mathrm{N}$ & 9 & B9 & 7 & A8 & 5 & D7 \\
\hline $\mathrm{H}$ & 10 & B10 & 9 & A9 & 7 & D8 \\
\hline $\mathrm{H}$ & 10 & B11 & 9 & A10 & 7 & D9 \\
\hline $\mathrm{N}$ & 9 & B12 & 7 & A11 & 5 & D10 \\
\hline $\mathrm{C}$ & 13 & B13 & 9 & A12 & 7 & D11 \\
\hline $\mathrm{C}$ & 2 & B14 & 1 & A13 & 14 & D12 \\
\hline $\mathrm{C}$ & 15 & B15 & 2 & A14 & 1 & D13 \\
\hline $\mathrm{H}$ & 16 & B16 & 15 & A 15 & 2 & $\mathrm{D} 14$ \\
\hline $\mathrm{C}$ & 16 & B17 & 15 & A16 & 2 & D15 \\
\hline $\mathrm{H}$ & 18 & B18 & 16 & A17 & 15 & D1 \\
\hline $\mathrm{C}$ & 18 & B19 & 16 & A18 & 15 & D1 \\
\hline $\mathrm{C}$ & 20 & B20 & 18 & A19 & 16 & D1 \\
\hline $\mathrm{O}$ & 21 & B21 & 20 & A20 & 18 & D1 \\
\hline $\mathrm{H}$ & 21 & B22 & 20 & A21 & 18 & D2 \\
\hline $\mathrm{H}$ & 21 & B23 & 20 & A22 & 18 & D2 \\
\hline $\mathrm{C}$ & 20 & B24 & 18 & A23 & 16 & D2 \\
\hline $\mathrm{H}$ & 25 & B25 & 20 & A24 & 18 & D2 \\
\hline $\mathrm{C}$ & 25 & B26 & 20 & A25 & 18 & D2 \\
\hline $\mathrm{H}$ & 27 & B27 & 25 & A26 & 20 & D2 \\
\hline $\mathrm{H}$ & 22 & B28 & 21 & A27 & 20 & D2 \\
\hline $\mathrm{C}$ & 1 & B29 & 2 & A28 & 3 & D27 \\
\hline $\mathrm{H}$ & 30 & B30 & 1 & A29 & 2 & D28 \\
\hline $\mathrm{H}$ & 30 & B31 & 1 & A30 & 2 & D29 \\
\hline $\mathrm{H}$ & 30 & B32 & 1 & A31 & 2 & D30 \\
\hline B1 & 1.3 & & & & & \\
\hline B2 & 1.30 & & & & & \\
\hline
\end{tabular}




\begin{tabular}{|c|c|}
\hline B3 & 1.389800 \\
\hline B4 & 1.419900 \\
\hline B5 & 1.229801 \\
\hline B6 & 1.399999 \\
\hline B7 & 1.000201 \\
\hline B8 & 1.380300 \\
\hline B9 & 1.339899 \\
\hline B10 & 1.010400 \\
\hline B11 & 1.009900 \\
\hline B12 & 1.329701 \\
\hline B13 & 1.359700 \\
\hline B14 & 1.349600 \\
\hline B15 & 1.400800 \\
\hline B16 & 0.999999 \\
\hline B17 & 1.400000 \\
\hline B18 & 1.000200 \\
\hline B19 & 1.400299 \\
\hline B20 & 1.509400 \\
\hline B21 & 1.430099 \\
\hline B22 & 1.001000 \\
\hline B23 & 1.000100 \\
\hline B24 & 1.400799 \\
\hline $\mathrm{B} 25$ & 0.999300 \\
\hline B26 & 1.400000 \\
\hline B27 & 0.999200 \\
\hline B28 & 0.950001 \\
\hline B29 & 1.420000 \\
\hline B30 & 1.070000 \\
\hline B31 & 1.070000 \\
\hline B32 & 1.070000 \\
\hline A1 & 113.990013 \\
\hline $\mathrm{A} 2$ & 103.940012 \\
\hline A3 & 130.460042 \\
\hline A4 & 128.739966 \\
\hline A5 & 111.410022 \\
\hline A6 & 117.310020 \\
\hline A7 & 125.240026 \\
\hline A8 & 116.010047 \\
\hline A9 & 126.990004 \\
\hline A 10 & 116.540048 \\
\hline A11 & 123.279986 \\
\hline A12 & 112.209975 \\
\hline A13 & 119.989992 \\
\hline A14 & 120.010002 \\
\hline A 15 & 131.571184 \\
\hline A16 & 119.989990 \\
\hline
\end{tabular}




$\begin{array}{lc}\text { A17 } & 119.999982 \\ \text { A18 } & 120.019992 \\ \text { A19 } & 120.029981 \\ \text { A20 } & 109.540034 \\ \text { A21 } & 109.470004 \\ \text { A22 } & 109.549980 \\ \text { A23 } & 120.000018 \\ \text { A24 } & 119.979996 \\ \text { A25 } & 119.980002 \\ \text { A26 } & 119.989952 \\ \text { A27 } & 109.470000 \\ \text { A28 } & 115.300174 \\ \text { A29 } & 106.697020 \\ \text { A30 } & 84.736433 \\ \text { A31 } & 133.178746 \\ \text { D1 } & -0.030000 \\ \text { D2 } & -179.970026 \\ \text { D3 } & -0.029986 \\ \text { D4 } & 179.970043 \\ \text { D5 } & 179.969989 \\ \text { D6 } & -0.100013 \\ \text { D7 } & 179.970032 \\ \text { D8 } & -0.740028 \\ \text { D9 } & -179.430001 \\ \text { D10 } & 0.029983 \\ \text { D11 } & -0.029922 \\ \text { D12 } & -179.816865 \\ \text { D13 } & -0.030040 \\ \text { D14 } & 25.479118 \\ \text { D15 } & -179.969992 \\ \text { D16 } & -179.969967 \\ \text { D17 } & -0.029961 \\ \text { D18 } & -179.969980 \\ \text { D19 } & 119.999995 \\ \text { D20 } & 0.029953 \\ \text { D21 } & -119.960011 \\ \text { D22 } & 0.049970 \\ \text { D23 } & 179.970009 \\ \text { D24 } & -0.049981 \\ \text { D25 } & -179.969996 \\ \text { D26 } & 179.970017 \\ \text { D27 } & -149.586445 \\ \text { D28 } & -153.676462 \\ \text { D29 } & 97.548958 \\ \text { D30 } & -14.326884\end{array}$




\section{Appendix B}

\section{Sample Amber MD Control File}

production MD run, 9.0 cut

\&cntrl

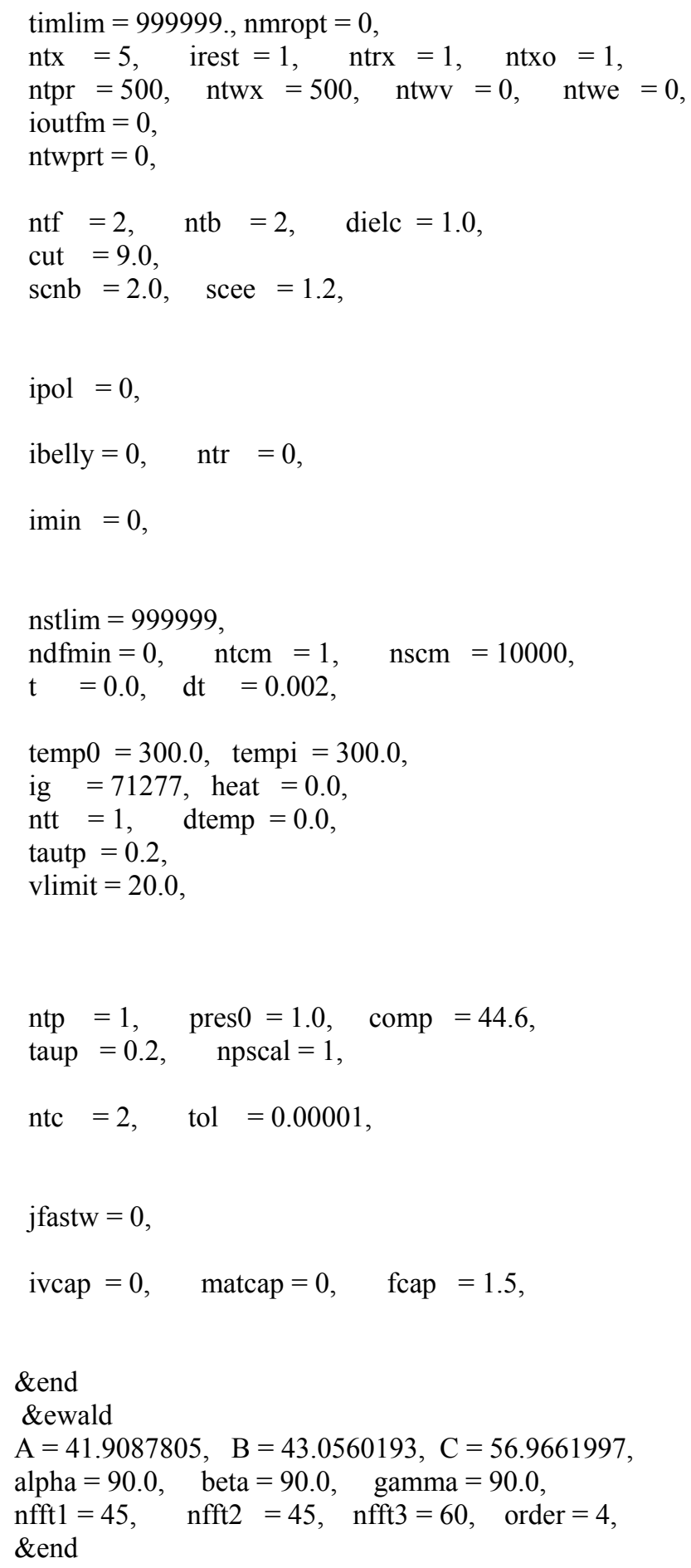




\section{Appendix C}

\section{Sample Amber MM-PBSA Control File}

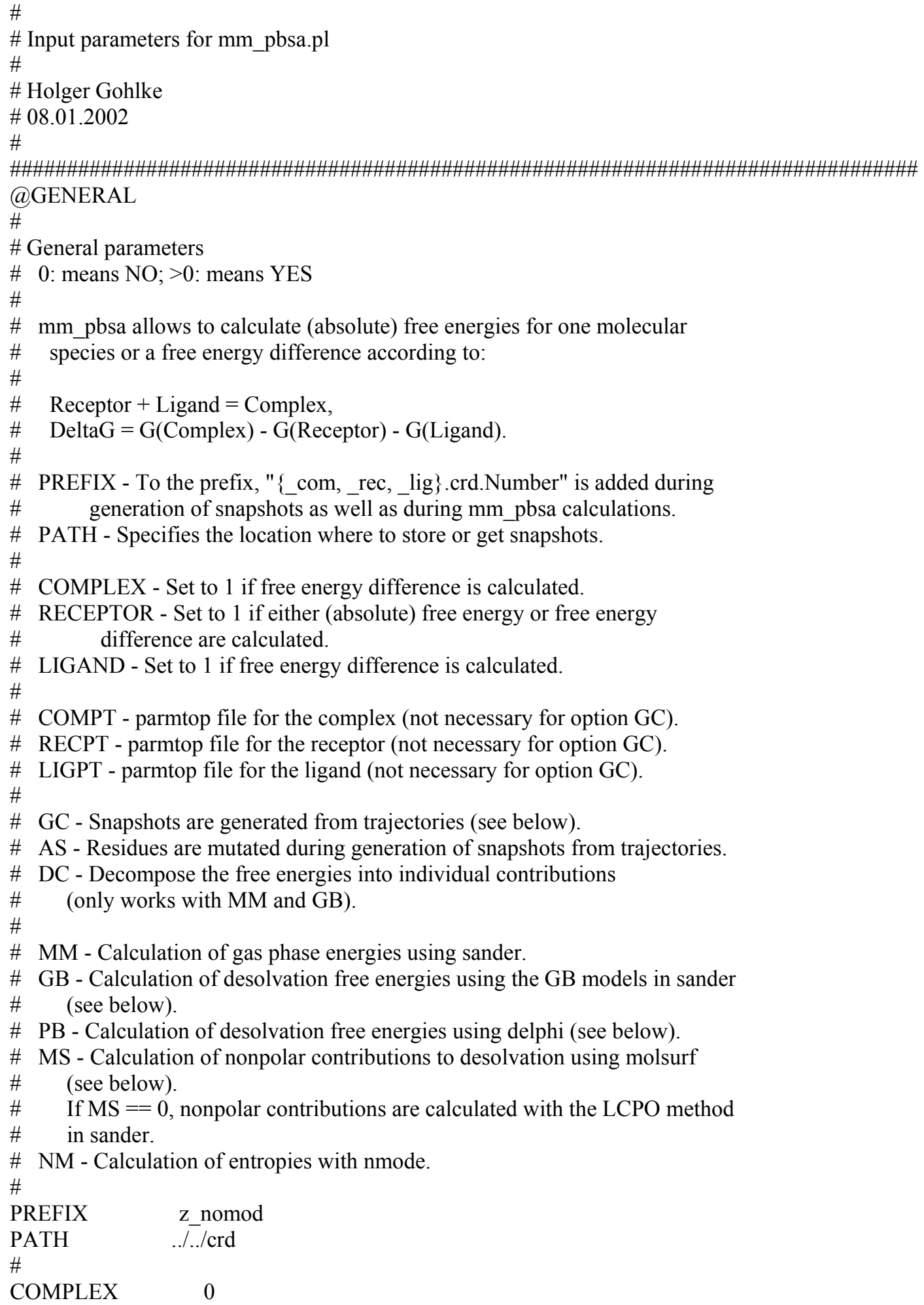




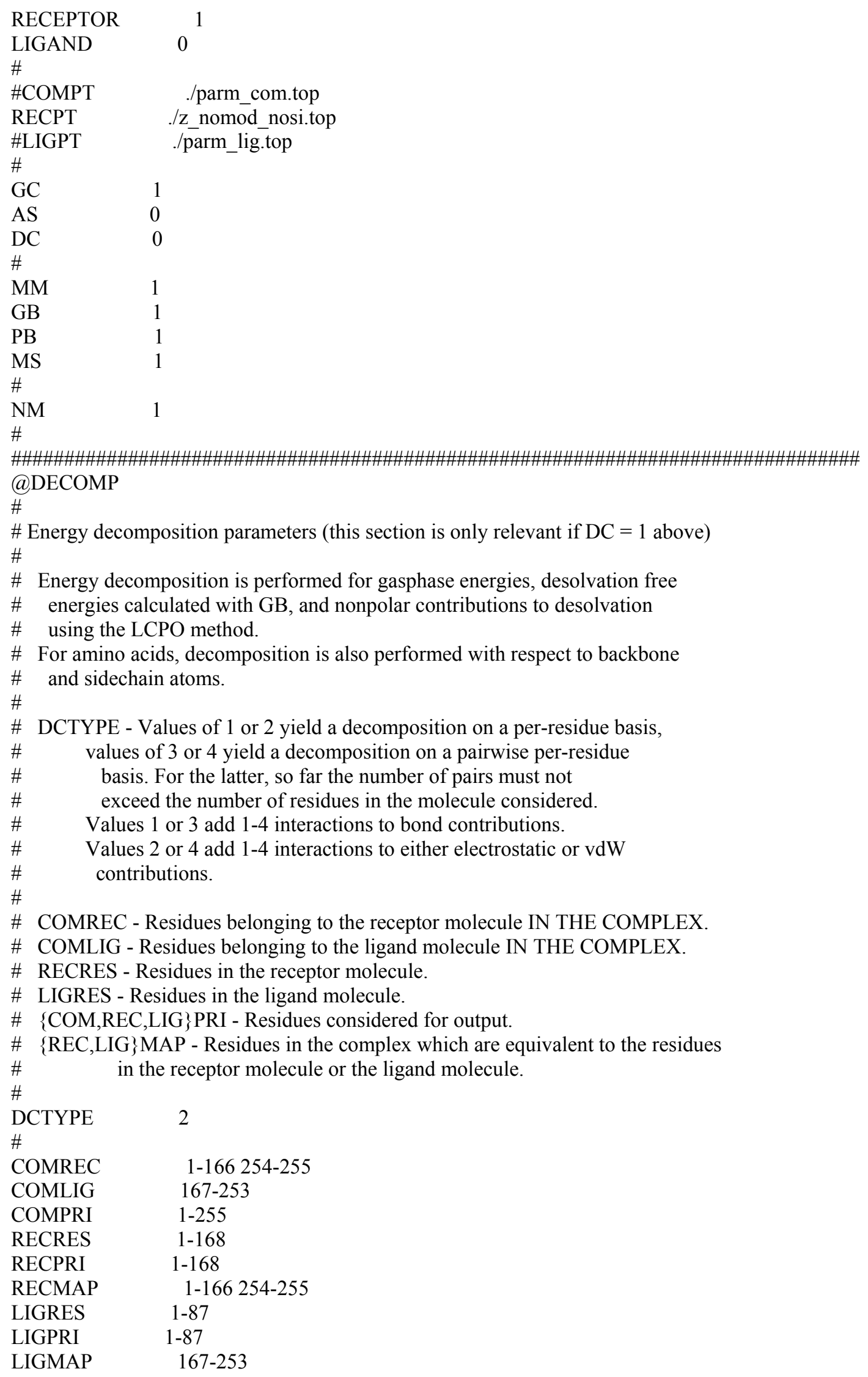




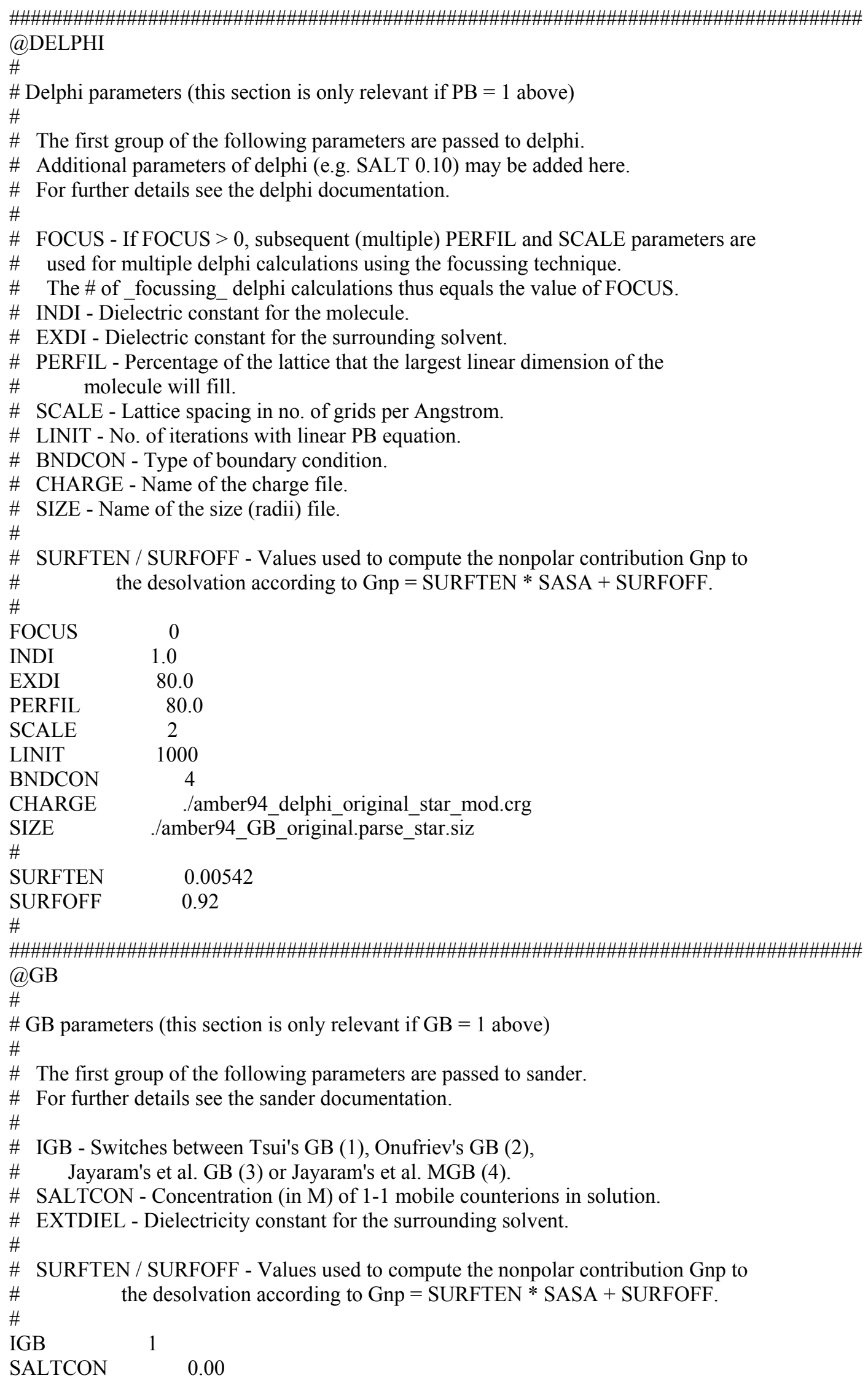




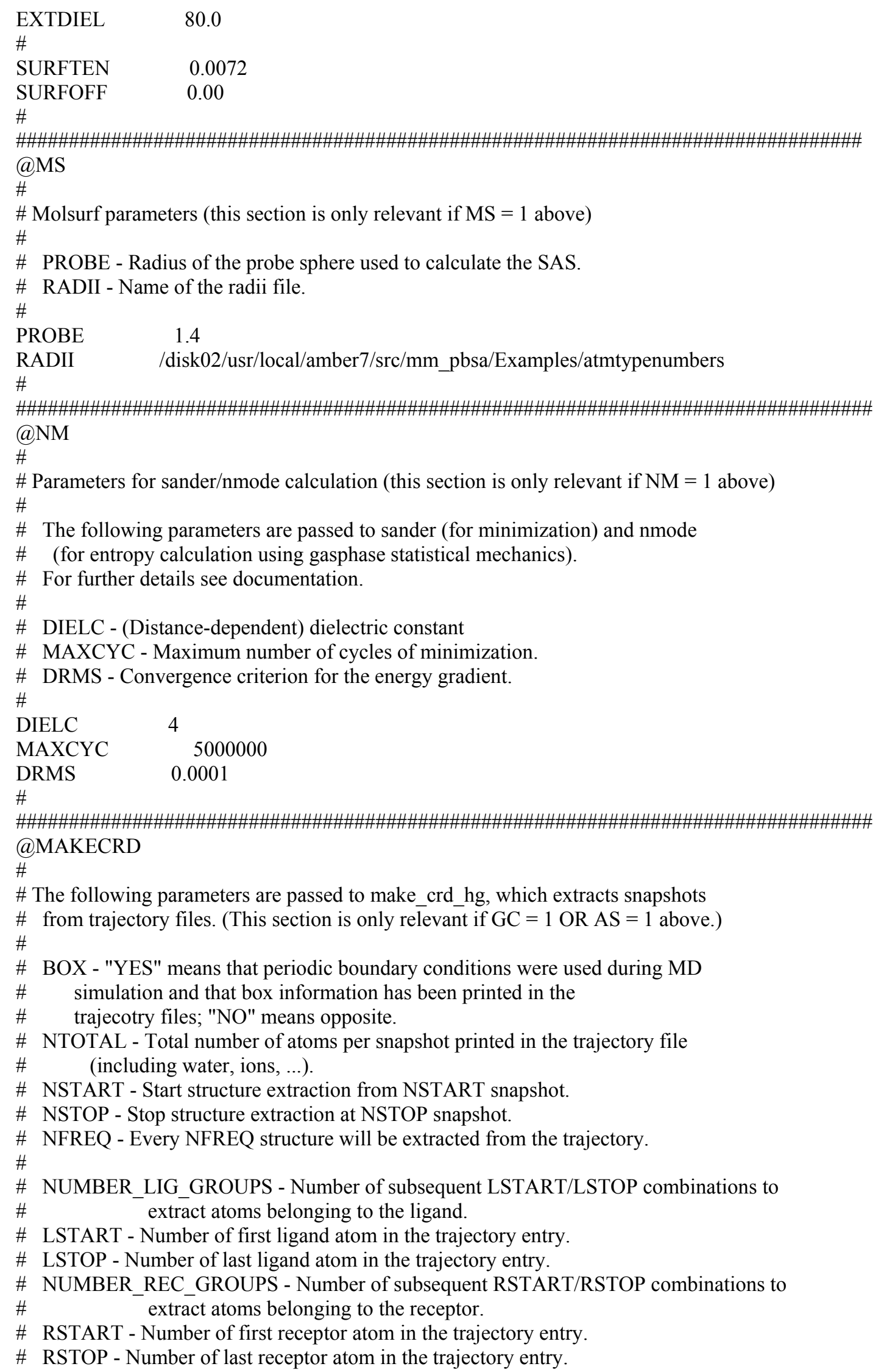




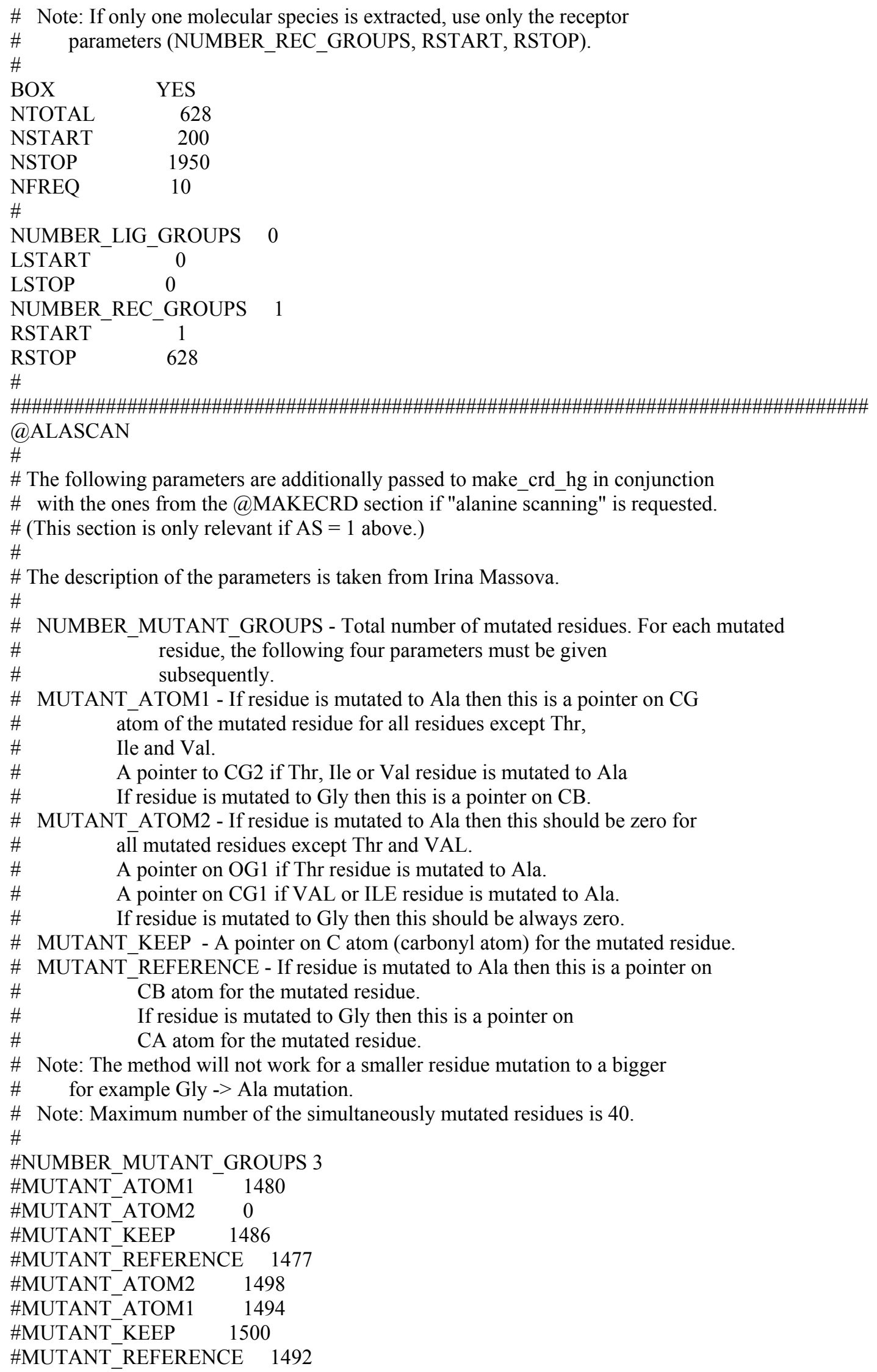




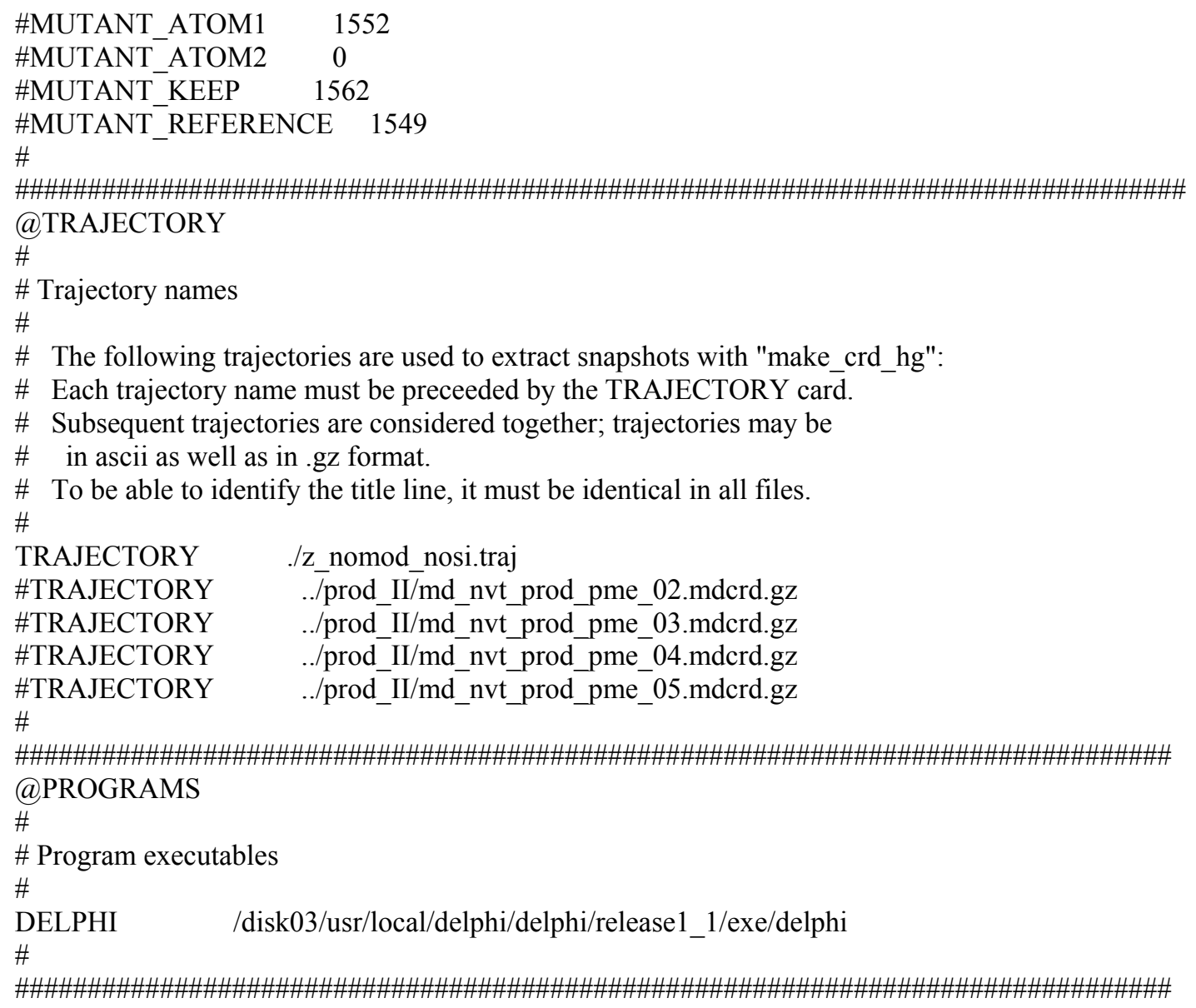

